\title{
Journal of \\ Children's Orthopaedics
}

European Paediatric Orthopaedic Society

\author{
EPOS $27^{\text {th }}$ Annual Meeting \\ 9-12 April 2008 \\ Warsaw, Poland
}

\section{Scientific Programme}

\&

Abstracts

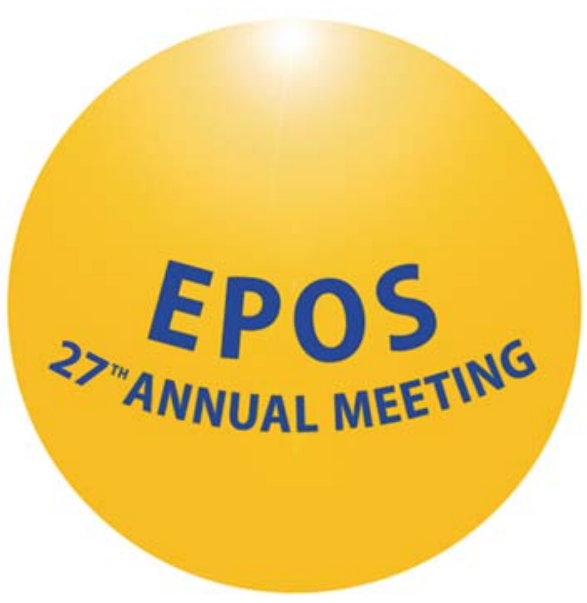




\section{Welcome to Warsaw}

\section{Dear Friends,}

I am very happy to see how Eastern Europe is becoming more and more involved in EPOS activities.

This year Warsaw will host the 27th EPOS Meeting, 20 years after the Budapest Meeting and 11 years after the Prague Meeting. Three EPOS-POSNA Instructional Courses have been recently organized in Croatia, Bulgaria and Romania. Moreover, new members from Eastern Europe are joining every year our Society and many of them are involved in the activities of EPOS Board. By hosting the most important EPOS event of the year, Warsaw will crown the magic moment of the renewed interest of Eastern Europe for Paediatric Orthopaedics. The pre-meeting program is very exciting: Dega osteotomy - that represents the pride of Pediatric Orthopaedic Surgery in Poland - will be largely illustrated, but the other classic pelvic osteotomies will be fully analyzed as well. Therefore the pre-meeting attendants will have an overall view of pelvic osteotomies with their specific indications, advantages, drawbacks and long-term results.

I also like to emphasize the high level of the scientific program of the Meeting that will start on Thursday, April 10th. Both oral presentations and posters have been carefully selected by our Reading Committee under the alert chairmanship of Muharrem Yazici who really did a good job. On behalf of EPOS, I also like to thank very much both Marek Napiontek and Jaroslaw Czubak for all their efforts to provide the Meeting participants an outstanding social program and a wonderful stay in Warsaw.

Professor Ernesto Ippolito

President of EPOS

\section{Dear Colleagues,}

For the first time the Annual Meeting of the European Paediatric Orthopaedics Society will be held in Poland, which is a visible sign of the times. Our country, member of the European Union since 2004 has been in the process of the economic growth. The old mixes with the new; history, often painful, with modernity and globalization. I am glad that right now, in a period of economic transformation and creation of a novel political culture, we have the honour to organize the 27th EPOS Meeting in Warsaw.

Warsaw, called Paris of the East before the Second World War reflects the history of our country, more than any other Polish city. The city is also a good starting point to visit our country, from North to South, from West to East. In the Northern part, the Baltic Sea with the historical Gdansk will ever be associated with Lech Walesa and Solidarnosc. In the South, Cracow and the Tatra Mountains represent the miniature of the Alps. In the west part, is the solid Greater Poland with Poznan, the first capital, where Professor Wiktor Dega lived and worked. And finally in the east of Poland, you can find splendid, unpolluted nature, very open people, a flavor of Orient and a certain unruliness of characters. All these make our country worth seeing.

The main topic of the meeting - Hip deformity in adolescence as well as the course - Dega osteotomy of the iliac bone are very special and we are sure that their levels will be up to your expectations. On behalf of the Polish Paediatric Orthopaedics Society I have a great pleasure of inviting you to Warsaw.

Professor Marek Napiontek

Chairman of the Organizing Committee 


\section{EPOS Executive Board}

\section{President}

Ernesto Ippolito

1st Vice-President

Andre Kaelin

2nd Vice-President

Dietrich Schlenzka

Former President

Shlomo Wientroub

General Secretary

Pierre Lascombes

Treasurer

Ruediger Krauspe

Councillors

Jiri Chomiak

Jaroslaw Czubak

Deborah Eastwood

Milan Kokavec

Muharrem Yazici

Scientific Committee

Fritz Hefti

Historian

Nando De Sanctis

EPOS News

Pavel Dungl

JCO

Shlomo Wientroub

Henry Bensahel

Education

Franz Grill

\section{Programme Committee}

Ernesto Ippolito

Ruediger Krauspe

EPOS Board Representative

Muharrem Yazici

Head of the Reading Committee

Marek Napiontek

Local host

\section{Reading Committee}

\section{Chairman}

Muharrem Yazici, Turkey

Members

Antonio Andreacchio, Italy

Reinald Brunner, Switzerland

Jaroslaw Czubak, Poland

Deborah Eastwood, United Kingdom

Mohammed El-Sobky, Egypt

Joel Lechevallier, France

Bjarne Moller-Madsen, Denmark

Gaetano Pagnotta, Italy

Jari Peltonen, Finland

Jan Poul, Czech Republic

Eitan Segev, Israel

Walter Strobl, Austria

Thomas Wirth, Germany

Reinhard Zeller, Canada

\section{Local Organizing Committee}

Marek Napiontek/ Chairman

Jaroslaw Czubak

Marek Józwiak

Wojciech Marczynski

Slawomir Snela

Marek Synder 


\title{
EPOS $27^{\text {th }}$ Annual Meeting \\ Pre-meeting Course
}

\author{
Wednesday, April 09, 2008
}

Hilton, Warsaw Hotel

DEGA OSTEOTOMY

\section{A. Dega Pelvic Osteotomy for DDH}

Wednesday, April 09

$13.30-13.45$

Acetabular development in normal hip and DDH: From birth to

maturity. Prof. Yucel Tumer

$13.45-14.00$

Differences in the transiliac osteotomies Dietrich Toennis

$14.00-14.15$

Discussion

$14.15-14.40$

$14.40-14.50$

$14.50-15.05$

$15.05-15.20$

$15.20-15.40$

\section{B. Other Applications of Dega Osteotomy}

$15.40-15.55$

25 years follow up of Dega osteotomy in CP patients

Scott J. Mubarak

15.55-16.10 Transiliac osteotomy in the treatment of dislocated hip in CP

Marek Jóźwiak

$16.10-16.25$

Combined treatment of LCP disease using DVO and Dega pelvic osteotomy Marek Napiontek

$16.25-16.40$

Long term results of the treatment of dysplastic hip in CP using Dega-like osteotomy

Reinald Brunner

$16.40-17.05$

Discussion

\section{Other Pelvic Osteotomy Techniques for Children}

17.05-17.20 Salter osteotomy: Long term results Simon Thomas

17.20-17.35 Pemberton osteotomy Perry L. Schoennecker

17.35-17.45 Age limit in pelvic osteotomies Dietrich Toennis

17.45-18.00 Discussion and adjournment 
Thursday, April 10

\section{EPOS $27^{\text {th }}$ Annual Meeting Scientific Programme}

08.00-08.09

Inauguration

Marek Napiontek, Local Hos

Ernesto Ippolito, President of EPOS

\subsection{9-10.20 Session 1. Hip in Adolescence}

Chairman: Ernesto Ippolito

Moderator: Jaroslaw Czubak

$08.09-08.29$

Plenary Lecture 1

Periacetabular osteotomy for adolescent hip dysplasia: Technical aspects and long term follow-up

Moritz Tannast

$08.29-08.49$

Plenary Lecture 2

Surgical dislocation

Michael Millis

$08.49-08.55$

1. Endoscopy assisted periacetabular osteotomy. A cadaveric study*

M. Inan, A. Gokçe, F. Ustunkan, H. Kucuk, I. Uzum

$08.55-09.01$

2. Ten year experience of periacetabular osteotomy in the treatment of hip dysplasia in adolescents**

J. Czubak, A. Niewiadomska, A. Sionek, M. Drwięga, M. Tyrakowski, S. Pietrzak, W. Przybysz

$09.01-09.09$

Discussion

$09.09-09.15$

3. Long-term results of Bernese periacetabular osteotomy to treat acetabular dysplasia M. B. Millis, C. Matero, D. Zurakowski, T. Matheney, Y. Kim

09.15-09.21 4. Changes in parameters describing gait in adolescents and young adults with dysplasia of the hip after Bernese periacetabular osteotomy. S. Pietrzak, A. Sionek, J. Czubak,

M. Syczewska

09.21-09.29 Discussion

Chairman: Mike Benson

Moderator: Pavel Dungl

$09.29-09.35$

5. Articulated hip distraction arthroplasty:

A treatment option for avascular necrosis of the child and adolescent femoral head

J. Gomez, D. P. Roye, D. S. Feldman, H. Matsumoto, H. J. van Bosse, J. E. Hyman, M. G. Vitale, S. Marangoz

*Predrag Klisic Best Basic Science Award nominee papers

**John Sharrard Best Clinical Science Award nominee papers
$09.35-09.41$

$09.41-09.49$

09.49-09.55

$09.55-10.01$

$10.01-10.07$

$10.07-10.20$

$10.20-10.50$

Coffee break

10.50-13.00 Session 2. Cerebral Palsy, Neuromuscular Disorders and Upper Extremities

Chairman: Reinald Brunner

Moderator: Marek Jozwiak

$10.50-10.55$

$11.01-11.09$

$11.09-11.15$

$11.15-11.21$
10. Botulinum toxin treatment of the biceps brachii muscle in children with cerebral palsy causes muscle tissue regeneration E.M. Pontén, S. Arnardottir, S. Gantelius

11. Long-term use of Botulinum Toxin type a (Btx-A) in children with cerebral palsy: Treatment consistency

G. Molenaers, A. Van Campenhout, E. Ortibus, J. De Cat, K. Desloovere, P. De Cock, P. Pauwels, V. Schörkhuber

\section{Discussion}

12. Distal rectus transfer in children with cerebral palsy. Does improvement persist long term?

F. Miller, A. Agarwalla, C. Church,

D. Thawrani, K. Dabney, N. Lennon, S. Marchesi

13. Treatment of stiff knee gait in children with cerebral palsy

A. Presedo, B. Ilharreborde, E. Morel, F. Fitoussi, G.F. Penneçot, M. Laassel, M. Mehrafshan, P. Souchet

Discussion 
11.29-11.35 14. Anthropometric measurements of growth of ambulant cerebral palsy children and adolescents

D.G. Pasparakis, C.M. Nestoridis, M.E. Pentarakis, M.N. Tziomaki,

N.G. Darras, N.M. Papandreou

11.35-11.41 15. Results of orthopaedic surgery when the surgeons follow the gait analysis recommendations in children with cerebral palsy

T. Terjesen, B. Lofterød

11.41-11.46 16. Effect of ankle foot orthoses on 3D trunk and pelvic motion during gait in children with cerebral palsy

A. Van Campenhout, E. Ortibus,

G. Molenaers, K. Desloovere,

P. Pauwels, P. Van de Walle

\subsection{6-11.59 Discussion}

Chairman: Tim Theologis

Moderator: Uri Givon

11.59-12.04 17. Characteristics of children with hip displacement in cerebral palsy

G. Hägglund, H. Lauge-Pedersen, P. Wagner

12.04-12.08 Discussion

12.08-12.14 Associations between orthopaedic findings, ambulation and health related quality of life in children with myelomeningocele

A. Danielsson, A. Bartonek, E. Levey,

H. Saraste, K. McHale, P. Sponseller

12.14-12.20 19. Orthopedic manifestations of congenital myotonic dystrophy during childhood

and adolescence

F. Canavese, M. D. Sussman

12.20-12.26 20. Slotted acetabular augmentation

for treatment of neuromuscular hip dysplasia in children and adolescents

M. Lugmair, C. Vuillerot, F. Chotel,

F. Sailhan, J. Berard, V. Cunin

\subsection{6-12.38 Discussion}

12.38-12.44 21. Operative treatment of the upper extremity in osteogenesis imperfecta

M. Rogalski, J. Zacher, M. Deja

12.44-12.50 22. Hoffer transfer may not achieve functional shoulder abduction and elevation in children with Erb's palsy

I. Ghanem, F. Dagher, K. Kharrat,

N. Choucair

\subsection{0-13.00 Discussion}

$13.00-14.00$

Lunch

$14.00-17.30$
Afternoon Tour
Friday, April 11

08.00-10.30 Session 3. Limb reconstruction, metabolic disorders, trauma and sports injuries

Chairman: Franz Grill

Moderator: Gamal Hosny

08.00-08.05 23. Prediction of limb length discrepancy during antenatal life. A radiographical study P. Kelly, A. Couture, A. Diméglio

08.05-08.09

Discussion

08.09-08.15 24. Femoral lengthening with the Albizzia nail

L. Peña, J. Monasterio, L. Arana,

O. Espinazo, R. Mongil

08.15-08.21 25. Congenital and acquired deformities in children corrected with the Taylor spatial frame

J. Horn, H. Steen, L. P. Kristiansen

08.21-08.27 26. Proximal femoral osteotomy in pediatric patients using monoaxial external fixation

R. Schiavon, A. Borgo, A. Micaglio, A. Rebeccato, S. Santini

$08.27-08.39$

Discussion

08.39-08.45 27. Corrective osteotomies and bone lengthening in $\mathrm{X}$-linked vitamin $\mathrm{D}$ resistant hypophosphatemic rickets (VDXLR)

L. E. Ramseier, G. Exner, S. F. Fucentese,

T. J. Neuhaus

08.45-08.51 28. Lengthening and axial correction of lower limb in Ollier disease

P. Koczewski, A. Koch, M. Jozwiak, M. Shadi

08.51-09.00 Discussion

\section{Trauma and Sports injuries}

Chairman: Pierre Lascombes Moderator:Manuel Cassiano Neves

09.00-09.05 29. Changes in paediatric trauma care A prospective epidemiological study. N. Francis, A. Diméglio, P. Kelly

09.05-09.09 Discussion

$09.09-09.15$

30. Treatment of radial neck fractures in children according to Métaizeau S. Endele, F. Fernandez, O. Eberhardt, T. Wirth

$09.15-09.21$

31. Physeal fractures of the distal radius and ulna: Long-term prognosis. F. Mancini, E. Ippolito, F. De Maio, V. Potenza 
$09.21-09.27$

32. Traumatic nerve lacerations

of the upper extremity in children

C. Plakogiannis, C. Papadopoulos,

D. Petratos, E. Manoukas,

G. Matsinos, I. Anastasopoulos

$09.27-09.39$

Discussion

$09.39-09.45$

$09.45-09.51$

$09.51-09.57$

$09.57-.10 .30$

$10.30-10.40$

\subsection{0-13.00 Session 4. Lower extremity and LCPD}

Chairman: Baxter Willis

Moderator: Hakan Omeroglu

\section{Lower extremity}

$10.40-10.45$

36. Fetoscopic release of amputation threatening extremity amniotic bands* F. Soldado, C. Amat, C. Fontecha, E. Carreras, J. Peiro, M. Aguirre, S. Arevalo, V. Martinez-Ibañez

10.45-10.51 37. Use of rhBMP-2 in congenital pseudarthrosis of the tibia B. S. Richards, C. E. Johnston, M. W. Shrader, R. D. Welch

\subsection{1-10.59 Discussion}

10.59-11.05 38. Development of human hip joint containment in second and third trimester of pregnancy. Cadaveric study* A. Masoń, A. Borowski, A. Grzegorzewski, J. R. Bowen, M. Synder, M. Topol

11.05-11.11 39. Accompanying pathologies, knee structures and treatment methods in congenital dislocation of the knee N. A. Okan, M. M. Orak

$11.11-11.19$

$11.19-11.25$

40. The treatment of the severe multi-planar deformity of the proximal part of the femur in children

A. A. Sakalouski, O. V. Kovalchuk, Y. V. Likcacheuski

11.25-11.29 Discussion

Chairman: Guy Fabry

Moderator: Marek Synder

\section{Legg Calve Perthes Disease}

$11.29-11.35$

$11.35-11.39$

$11.39-11.45$

$11.45-11.51$

$11.51-11.59$

$11.59-12.05$

$12.11-12.17$

$12.17-12.13$

$12.13-12.40$

$12.40-13.00$

$13.00-14.20$
41. Increased frequency of PAI-1 gene polymorphism in Legg- Calvé-Perthes disease: Preliminary results of a pilot study

D. Yazgan Aksoy, A. Gurgey, I. C. Haznedaroglu, M. Alpaslan, M. C. Aksoy

Discussion

42. Presentation and outcome of conservatively treated patients with Legg- Calvé-Perthes disease L. Froberg, F. Christensen, N. Wisbech Pedersen, S. Overgaard

43. Late onset Perthes disease T. Wirth

Discussion

44. Surgical treatment of Legg-Calvé-Perthes disease: Improved results for severe forms Catterall IV/Herring $\mathrm{C}$

A. Diméglio, F. Canavase, M. Ali, P. Kelly

45. Legg-Calvé-Perthes disease -The acetabular development following pelvic osteotomy.

A. J. Schreiner

46. Triple pelvic osteotomy in Legg-Calvé-Perthes disease using a single incision: A four-year review E. Conroy, D. McCormack, J. Harty, M. Timlin

47. Chiari osteotomy and shelf acetabuloplasty in the treatment of severe Perthes disease M. Batouty, W. Adl, Y. Kandeel

Discussion

Ceremony for Maximus Meritis awards and Honorary Members

Lunch 


\subsection{0-16.20 Session 5. Spine and Infection}

Chairman: Andre Kaelin

Moderator: Hans Pruijs

14.20-14.40 Plenary Lecture 3

Botulinum Toxin-A in the treatment of gait problems in cerebral palsy. Past, Present and Future

Guy Molenaers

\section{Spine}

14.40-14.45 48. Experimentally-induced scoliosis by costotransversectomy in mini pigs. Two models of spinal tensegrity breakage comparing midline versus posterolateral surgical approach*

O. Riquelme-García, A. González-Miranda, C. Barrios, C. Correa, E. Hevia, J. Burgos, J. González-López

14.45-14.51 49. Growing rod instrumentation and vertebral body growth. A radiological investigation in immature pigs*

G. Yilmaz, C. Ozkan, G. Demirkiran,

G. Huri, K. Daglioglu, M. Yazici

14.51-14.57 50. Fetal surgery of myelomeningocele by fetoscopy in the ovine fetus*

C. Fontecha, F. Soldado, J. Peiro, J. Sevilla, M. Aguirre, V. Martinez-Ibañez

14.57-15.09 Discussion

15.09-15.15 51. Natural history of early thoraco-lumbar kyphosis in children with achondroplasia

W. G. Mackenzie, A. Littleton,

B. Borkhuu, G. Chan

15.15-15.21 52. Scoliosis associated with congenital diaphragmatic agenesia. Report of a large series (41 cases)

A. Antaranyan, B. Dohin , C. Garin,

D. Gorduza, R. Dubois, R. Kohler

15.21-15.29 Discussion

Chairman: Dietrich Schlenzka

Moderator:Muharrem Yazici

15.29-15.35 53. Surgical correction of spinal deformities after heart transplantation: A case series report

D. P. Roye, B. Kaufman, J. A. Gomez,

J. E. Hyman, L. Addonizio, M. G. Vitale

15.35-15.51 54. Vertebral manifestations in CRMO

M. Langendoerfer, F. Fernandez, T. Hospach,

T. Wirth

15.21-15.29 Discussion

\section{Infection}

15.59-16.05 55. Prospective study into short-term intravenous antibiotic treatment of acute septic arthritis and acute osteomyelitis in children

R. Kanwar, C.E. Bache, H.K. Graham

16.05-16.11 56. Coxaplasty in hip reconstruction for type II late sequelae of septic epiphysitis:

A preliminary report

H.M. El Tayeby

16.11-16.20 Discussion

16.20-16.30 Coffee

\subsection{0-18.00 Session 6. Tumors}

Chairman: Gerard Bollini

Moderator: Bjarne Moller-Madsen

16.30-16.35 57. Musculoskeletal Langerhans cell histiocytosis in children: analysis of patients over three decades.

J. P. Dormans, A. Arkader, H. Hosalkar,

L. Moroz, M. Glotzbecker

16.35-16.41 58. Eeosinophilic granuloma of the bone: A diagnostic issue

A. Andreacchio, A. Brach del Prever,

A. Linari, A. M. Postini, C. Defilippi,

M. Berta, M. Chiavola, M. Pagano

16.41-16.49 Discussion

16.49-16.55 59. Diagnosis of osteoid osteomas with a 3D time-resolved MRI angiography P. Journeau, A. Lecocq, L. Mainard-Simard, M. Claudon, P. Lascombes, T. Haumont

16.55-17.01 60. Percutaneous resection of osteoid osteoma (73 cases). A 20 year experience

R. Kohler, B. Dohin, I. Canterino

17.01-17.09 Discussion

Chairman: Shlomo Wientroub

Moderator: Ruediger Krauspe

17.09-17.15 61. Epiphysial sparing techniques for malignant tumors in children using epiphysial distraction (Pamplona technique) and transepiphysial resections

G.U. Exner, S. Jürgen

17.15-17.21 62. Diaphyseal reconstruction after tumoral resection of long bones in children using induced membranes technique. Preliminary report of 5 cases

B. de Billy, B. de Courtivron, C. Bonnard, J. Langlais, M. L'Kaissi, M. Planchenault 
$17.21-17.27$

63. Complications of extendable prosthesis in children who survive longer than 10 years F. Chotel, 1. Jeys, R. Grimer, R. Tillman, S. Abudu, S. Carter

17.27-17.33 64. Management of limb deformities following use of vascularised fibula after tumour resection in skeletally immatures H. Abdel-Ghani, H. El-Barbary, W. Ebeid

\subsection{3-17.49 Discussion}

17.49-17.55 65. Results of treatment of Ewings sarcomas at a single institution in Switzerland

A. Krieg, F. Hefti, M. Paulussen

17.55-18.00 Discussion

20.00

Conference Dinner

Saturday, April 12

08.00-10.00 Annual General Assembly

10.00-10.30 Coffee

\subsection{0-13.00 Session 7. DDH and Foot deformities}

Chairman: Nando deSanctis

Moderator: Tomas Epeldegui

$10.30-10.50$

Plenary Lecture 4

Radial longitudinal deficiency

Leszek Romanowski

\section{$D D H$}

$10.50-10.55$

66. Discrete event simulation for policy decision making and optimized implementation in screening strategies for developmental dysplasia of the hip

S. Ramwadhdoebe, C. P. Uiterwaal,

E. Buskens, R. J. Sakkers

$10.55-11.01$

67. Bilaterally dislocated hips treated with the Pavlik harness are not at higher risk for failure

A. Borowski, A. Littleton, D. Thawrani, L. Grissom, M. Thacker

Discussion

11.09-11.15 68. The role of acetabular cartilage thickness on acetabular development in developmental dysplasia of the hip

O. Kalenderer, A. Bicimoglu, H. Agus,

H. Omeroglu, Y. Tumer

11.15-11.21 69. Evaluation of isokinetic muscle strength measurements in patients with unilateral developmental dysplasia of the hip treated with one-stage combined procedure

S. Yilmaz, A. Bicimoglu, E. Aksahin,

H. Muratli, H. Y. Yuksel, L. Celebi, M. Ersoz
$11.21-11.27$

Chairman: Henri Bensahel

Moderator: Marek Napipontek

\section{Foot deformities}

$11.39-11.45$

$11.45-11.51$

$11.51-11.59$

$11.59-12.05$

$12.05-12.11$

$12.11-12.19$

$12.19-12.23$

$12.23-12.29$

$12.35-12.47$

$12.47-13.00$

Discussion V. de Rosa

Discussion R. Seringe

Discussion study

Discussion
70. The MRI evaluation of iliopsoas and other hip muscles in patients with unilateral DDH treated with one-stage combined procedure H.Y. Yuksel, A. Bicimoglu, E. Aksahin, H. Muratli, L. Celebi, S. Duran, S. Yilmaz

71. Children tarsal coalitions: Results of resection-fat interposition

C. Bonnard, B. de Courtivron, C. Coulibali, F. Bergerault, L. le Nail, M. Planchenault

72. Talonavicular arthrodesis in patients with neurological disorders and flat foot deformity G. de Coulon, A. Kaelin, D. Ceroni,

73. Assessment of the result of club foot treatment : a new quantified kinematic method W. P. Wicart, B. Maton, J. Dubousset,

74. Leg muscle atrophy in idiopathic congenital clubfoot: Is it primitive or acquired?

F. De Maio, A. Orefice, E. Ippolito, F. Mancini, V. Potenza

75. Phases in gap healing following Ponseti type achilles tenotomies. An ultrasonographic

R. Kanwar, H. Prem, K. Johnson

76. The effectiveness of Ponseti method after failed orthopedic treatment

A. M. Ey Batlle, C. Alves, J. A. Abad

77. Early results of the Ponseti method for treatment of arthrogrypotic clubfoot S. Boehm, F. Alaee, M. Dobbs, M. F. Sinclair, N. Limpaphayom

Ceremony for Sorrento awards, Announcement of 2008 awards Adjourn 


\title{
EPOS - 27th Annual Meeting
}

\author{
Warsaw，9-12 April 2008
}

\section{ORAL PRESENTATIONS}

\section{1 \\ ENDOSCOPY ASSISTED PERIACETABULAR OSTEOTOMY: A CADAVERIC STUDY}

\author{
M. Inan, A. Gokçe, F. Ustunkan, H. Kucuk, I. Uzum
}

Purpose: The periacetabular osteotomy (PAO) was described for reconstruction of symptomatic dysplastic hips in adolescents and young adults. We believe that PAO may be performed with a mini incision under direct endoscopic control and the purpose of this study was to describe a minimal invasive periacetabular osteotomy technique by using endoscopy.

Methods: The three dimensional (Tri-D) computerized tomography (CT) of 14 patients by using a computer model of the pelvis were analyzed to identify safe zone of the periacetabular bone cut. By using these measurements, special curve shaped guides and osteotomes were developed. Four fresh-frozen cadaver pelves were used for this study.

Results: According to Tri-D CT measurements, the direction of the osteotome should not be exceeded more than $26^{\circ}$ in coronal plan and $12^{\circ}$ in transverse plan. No damage to vital structures was identified in any of the dissected cadavers. PAO was achieved without intraarticular fracture.

Conclusions: As a result, the current study demonstrated that PAO may be performed with a mini incision under direct endoscopic control. However, to suggest this procedure, animal study and clinical experience should be performed in detail.

Significance: The current study is the a preliminary study showing a periacetabular osteotomy technique assisted endoscopically, which can be a model for further study.

\section{2}

\section{TEN YEAR EXPERIENCE OF PERIACETABULAR OSTEOTOMY IN THE TREATMENT OF HIP DYSPLASIA IN ADOLESCENTS}

\section{J. Czubak, A. Niewiadomska, A. Sionek, M. Drwięga,} M. Tyrakowski, S. Pietrzak, W. Przybysz

Purpose: The aim of the study is to present our 10 years of experience and results of using PAO in the treatment of hip dysplasia in adolescents.
Methods: In the years 1998-2007, 362 PAO were performed in our hospital. In this group 40 girls and 3 boys were operated on the second decade of life (49 hips). The age at the time of PAO ranged from 12,6 to 19,5 years, av.16.8 years. PAO was the primary treatment in 27 cases. 16 patients the previously been operated in childhood for the treatment of hip deformity. The main indication for PAO was hip dysplasia with different degrees of subluxation. In this group the PAO was a single procedure. The previous treatment consisted of: open reduction in 10 hips, DVO in 14 hips, pelvic osteotomy (Salter, Dega, Chiari) in 8 hips, combined: open reduction + DVO + pelvic osteotomy in 10 hips, greater trochanter transfer in 3 hips, bone lengthening in 4 hips, acetabular cyst removal in 1 hip. The follow-up period ranged from 1 to 7,5 years av. 2 years. There were also hip deformity connected with sequaelae of septic artitis (3 hips), Perthes (2 hips) and CP (2 hips). In 31 hips the Smith-Petersen, and in 12 hips ilioinguinal approach were performed. In clinical pre-op, and post-op examination the following factors were considered: pain, limping, Trendelenburg sign, range of motion, length discrepancy. Radiographic pre-op and post-op examination consisted of: AP view of the pelvis, false profile and AP view with the leg in abduction. Classic and anterior CE angle were measured. Harris Hip Score and WOMAC classification were used at the last examination.

Results: Flexion slightly decreased from pre-op. $90-140^{\circ}$ (av. $118^{\circ}$ ) to $80-130^{\circ}$ (av. $104^{\circ}$ ) post-op. abduction remained unchanged 15-60 (av. $40^{\circ}$ ) and $15-60^{\circ}$ (av. $40^{\circ}$ ) respectively, adduction

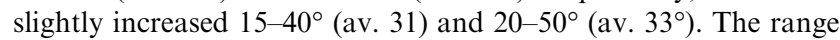
of rotation did not change after the operation. The sign of Trendelenburg was found in 27 hips before operation and in 8 hips post-op. Pain ocurred in 40 hips before operation and in 4 hips after the surgery. Both classic and anterior $\mathrm{CE}$ angle increased after the surgery in all cases from $-14^{\circ}$ to $34^{\circ}$ and from $-10^{\circ}$ to $35^{\circ}$, respectively. In 8 hips the following complications occurred: 1 overcorrection, 2 insufficient corrections, 2 urinary tract infections, and ectopic bone formation in 3 hips. We did not find any signs of AVN and infection of bone or soft tissue in our series. The av. time of operation and av. blood loss was not significantly different in comparison with older group treated with using PAO as a primary operation.

Conclusions: In cases previously operated and in males we strongly recommend Smith-Petersen approach extended into the frontal part of the hip.

Significance: We find the PAO as a safe and effective tool for treating hip joint pathology increasing treatment possibilities for hip joint preservation. 
3

\section{LONG-TERM RESULTS OF BERNESE PERIACETABULAR OSTEOTOMY TO TREAT ACETABULAR DYSPLASIA}

\author{
M. B. Millis, C. Matero, D. Zurakowski, T. Matheney, Y. Kim
}

Purpose: Report minimum 5 year results for Bernese PAO in treating symptomatic acetabular dysplasia.

Methods: Of more than 800 hips treated by us for symptomatic dysplasia since 1991, 160 hips with classic DDH were located and 135 were evaluated more than 5 years after surgery with WOMAC scores in all an radiographs and physical examination in most. PAO was done through an abductor-sparing direct anterior approach in $153 / 161$. Arthrotomy was performed in most. Solid internal fixation with multiple screws allowed early mobilization to crutches without casting. Age at surgery ranged from 9 to 48 years (mean 24). Followup was from 5 to 15 years(mean 9.5).

Results: 118 hips $(87 \%)$ were still preserved at mean of 9.5 years after surgery, with mean WOMAC pain score 4 out of 20 (good to excellent). 17 hips underwent THR at a mean of 6.1 years after PAO. Kaplan-Meier survivorship was $84 \%$ at 10 years. Multiple logistic regression analysis suggested 2 independent factors for conversion to THR: (1) Age at surgery $>35$ year (2) Preop joint space width $<2 \mathrm{~mm}$. With neither failure predictor present, failure risk was $4 \%$. With 1 predictor present (either age $>35$ OR joint space $<2 \mathrm{~mm}$ ), failure risk was $35 \%$ with both present, failure risk was $75 \%$.

Conclusions: Bernese PAO was effective in relieving symptoms and preserving function in the large majority of treated patients at a mean of 9 years after surgery. Even with some arthrosis, and even with some arthrosis in older patients, most hips did well. Either severe arthrosis or the combination of age $>35$ year and arthrosis should lead to caution in considering PAO to treat the symptomatic dysplastic acetabulum.

Significance: The large incidence of hip dysplasia, and its associated risk of causing osteoarthrosis, should lead to increased timely use of proven effective joint-preserving procedures such as PAO both to correct symptoms and preserve the hip.

\section{4}

\section{CHANGES IN PARAMETERS DESCRIBING GAIT IN ADOLESCENTS AND YOUNG ADULTS WITH DYSPLASIA OF THE HIP AFTER BERNESE PERIACETABULAR OSTEOTOMY}

\section{S. Pietrzak, A. Sionek, J. Czubak, M. Syczewska}

Purpose: Aim of the study was to describe changes in the gait pattern occuring in patients after Bernese periacetabular osteotomy performed because of hip dysplasia. The second one was to establish if there is any correlation between the final clinical assessment, morphological changes in the hips described by radiographic parameters and gait pattern changes in these patients after the surgery.

Methods: Records of 29 patients (4 men and 25 women) with dysplasia of the hip were reviewed. 25 patients had unilateral and 4 bilateral involvement of the hip. The mean age at surgery was 27 years (from 15 to 49), the mean follow-up was 18 months. Data prior to surgery and from the final examination were compared. Clinical examination included patients' complaints con- cerning pain, function, limping (acc. to Harris hip score form), range of motion and leg length. Radiological examination included center-edge angle (CE), migration percentage index (MPI) and anterior coverage angle (false profile-FP). The gait analysis was performed using six camera VICON 460 systems. Simultaneously the ground reaction forces (GRF) were recorded using Kistler force plates. Data from gait kinematics, GRF reaction forces were collected for statistical analysis. Wilcoxon test, Spearman rank order correlations and Student's $t$-test with a $P=0.05$ level of significance were used.

Results: A changed (in respect to normal) gait pattern was observed in majority of our patients before the surgery (step length, $\%$ of the stance phase altered significantly at the affected side; decreased pelvic tilt, increased pelvic obliquity, decreased hip range of motion in the sagittal and horizontal plane). After the surgery most of the temporo-spatial parameters became symmetrical on both sides. The kinematic data did not show an evident return into the normal pattern. FP is the only radiographic parameter which correlated with the intensity of patients' complaints and change of some of the gait parameters pre- and post-op.

Conclusions: The gait pattern observed in our patients prior to the surgery did not change at regular and evident way as it could be expected regarding the improvement of patients' radiographs and disappearance of their complaints. The changed gait pattern could be explained as a compensatory mechanism, perhaps of an antalgic character before the surgery, but in the follow-up although the hip improved, the gait remained disturbed, especially in the kinematic parameters.

Significance: Although the results of Bernese acetabular osteotomy are widely documented, there are few studies trying to describe the changes of the gait pattern in those patients.

\section{5}

\section{ARTICULATED HIP DISTRACTION ARTHROPLASTY: A TREATMENT OPTION FOR AVASCULAR NECROSIS OF THE CHILD AND ADOLESCENT FEMORAL HEAD}

J. Gomez, D. P. Roye, D. S. Feldman, H. Matsumoto, H. J. van Bosse, J. E. Hyman, M. G. Vitale, S. Marangoz

Purpose: To describe the clinical outcomes of adolescent patients treated with hip distraction arthroplasty for avascular necrosis (AVN) of the femoral head. Outcomes were examined in order to better understand the usefulness and indications of performing hip arthrodiastasis in this patient population.

Methods: Retrospective review was performed on 31 hips with femoral head AVN treated with hip distraction arthroplasty. Mean age at treatment was 14.2 years. Preoperative and follow-up pain, and physical limitations, as well as follow-up range of motion (ROM) were assessed.

Results: Follow up assessment was obtained at 18.3 years of age. Time of follow up was 55.3 months after distraction. The etiologies of AVN were: 11 Slipped Capital Femoral Epiphysis (SCFE); 5 Idiopathic AVN; 3 with hip dysplasia; and 12 others. There was a significant difference in pain preoperatively and postoperatively $(P<0.001)$, the majority of patients $(79.5 \%, n=23)$ had less pain after the treatment. Multivariate regression model demonstrated that patients with SCFE were likely to have less improvement in pain than patients with other etiologies (odds ratio: $24.8 ; P=0.035$ ). All patients had activity limitations be- 
fore the treatment; at the postoperative assessment more than half of our patients $(n=15)$ reported no limitations in their activities. Nine patients had minor complications with the fixator. At follow up, $5(16.1 \%)$ patients converted to total hip replacement or arthrodesis.

Conclusions: Hip distraction arthroplasty in adolescent patients with symptomatic AVN reduces the amount of pain and limitation in daily activities, at a follow up of 4.6 years. Arthrodiastasis is not the final solution to AVN, with longer follow up patient's symptomatology increases. Patients with AVN secondary to SCFE do not beneficiate of this procedure as much as other patients do. Hip distraction is a safe and appropriate procedure to perform in these patients. The procedure might be able to delay definitive surgical procedures at an early age, restoring function and improving the patient $\mathrm{s}$ quality of life.

Significance: This study confirms safety and implies efficacy. Complications were those associated with external fixators and with the osteotomies used to treat proximal femoral deformity. None of the reported complications had lasting impact on patient outcome. This review demonstrated that hip arthrodiastasis was safe, able to improve pain, able to improve function and was likely to delay the need for total hip or similar procedures in young patients.

\section{6}

\section{PELVIC SUPPORT OSTEOTOMY}

\section{G. Hosny}

Purpose: Hip instability in the young age is difficult to treat especially if it is accompanied by pain \& bone shortening. Subtrochanteric valgus osteotomy to support the pelvis was suggested in 1838.Ilizarov popularized the idea by adding another distal femoral osteotomy to correct the mechanical axis and compensate for length inequality. The aim of this study is to evaluate the rsults of this operation highlighting the predictability of the results.

Methods: From 1993 till 2005, 39 cases had been treated by pelvic support osteotomy in our institution.5 cases were excluded due to inadequate follow up data. Age of patients ranged from 10 to 26 years [average $14.5 \mathrm{y}$ ].There 20 females and 14 males. The operation was performed on the right side on 19 cases. The etiology was in 7 cases, paralytic hip dislocation in 9 cases, septic arthritis in 10 cases, OA in 2 cases, neglected fracture neck femur in 2 cases, proximal femoral focal deficiency in 3 cases and $\mathrm{TB}$ in one case. External fixation time ranged from 4-12 $\mathrm{m}$. All patients had a positive Trendeleburg test and 31 patients had hip pain preoperatively. Evaluation parameters include were pain, Trendelenburg test, limping, walking distance, ROM, L.L discrepancy and satisfaction of the patients.

Results: After an average follow up 6.5 y [1-13 y] there were 6 excellent, 23 good and 5 poor resuts. Trendelenburg sign disappeared in 20 patients. Pain disappeared in 28 patients. Magnitude of lengthening ranged from 3.5 to $12 \mathrm{~cm}$. Complications included: Somesort of pin tract infection in all cases, knee stiffness in 3 cases, fracture of the regenerate in 4 cases and knee subluxation in one case.

Conclusions: Early results of Ilizarov modification of pelvic support osteomy may be encouraging. However, the possibility of deterioration with time and the unpredictability of improvement of Trendelenburg gait should be considered.

Significance: Pelvic Support osteotomy is a salvage procedure with limited indications and guarded results.

7

\section{THREE-DIMENSIONAL (3-D) SHAPE OF GROWTH PLATE OF PROXIMAL FEMUR IN CHILDREN AND ITS SIGNIFICANCE IN ETIOLOGY OF SLIPPED CAPITAL EPIPHYSIS}

\author{
G. Kandzierski, A. Wójcik, J. Kaakucki, M. Kalita, \\ S. Piszczatowski
}

Purpose: The paper underlines age related changes of dimensional shaping (3-D) of growth plate of proximal femur and their significance in etiology of slipped capital epiphysis.

Methods: The research was based on X-ray, CT and IMR examination (total n. 180) of normal hips joints of children aged $2-12$ years. The age related changes in dimensional shaping of growth plate of proximal femur were analyzed. Moreover the authors conducted the X-ray, CT and IMR examination of "in vitro" specimens of proximal femurs acquired from human corpses ( 2 children). At all specimens the shape of growth plate was visualized by separation of femoral head and neck. Acquired data was used to elaborate biomechanical models of three dimensional distributions of stress forces reacting on the growth plate.

Results: The growth plate of proximal femur in children shows changeability of dimensional shape according to the age of child. The largest pleating is in age of 5-8 years and it is very specific. Apart from sinuous shape of the whole surface of growth plate there are numerous smaller immersions and eminences. The whole surface of the growth plate is thus larger than the actual diameter of femoral head. The type of pleating allows firm and efficient junction of femoral head and neck. The same phenomenon is known to carpenters while joining two layers of wood. The same pleating of growth plate is present at other long bone ends. It is known that total epiphyseal slipping is rather observed in very young children (23 year of age) when the growth plates are thick and without pleating. In older (over 5 years) children the slipping of physis is usually accompanied with fracture of metaphysis or/and epiphysis. After 10th year of life the growth plate of proximal femur in children gradually becomes more spherical and the lack of pleating (more regular shape) also enables occurrence of slipped capital epiphysis. The direction of slipping is dependable on the direction of reacting forces and "inconvenient mechanically" shaping of growth plate in children after 10th year of life. The femoral head is displaced backward and downward-which is caused by cutting forces reacting during the flexion of femur in the hip joint.

Conclusions: The changes of dimensional shaping of epiphyseal growth zone from pleated to more spherical enables occurrence of slipped capital epiphysis in children above 10th year of life.

Significance: The results of present study attribute to explanation of etiology and pathomechanics of slipped capital epiphysis. 
8

\section{TREATMENT OF CHRONIC ADOLESCENT EPIPHYSIOLYSES BY MEANS OF TRIDIMENTIONAL INTERTROCHANTERIC OSTEOTOMY}

\section{Georgiev, B. Vladimirov, I. Efremov, R. Kehayov}

Purpose: For an over 15 year period 42 cases of chronic adolescent epiphysiolysis with moderate severity have been treated and followed in the Department of Pediatric Orthopedics, Medical University - Sofia. As such we accepted the epiphysiolysis with posterior tilt angle (PTA) between 30 and $55^{\circ}$ and duration of complaints more than a month.

Methods: In all of them an original Bulgarian technique of tridimentional intertrochanteric osteotomy after Holevich-Djerov has been applied. In this technique the correction is achieved by a double angulated individual plate with built-in angles of correction of retroversion and varus dislocation of the epiphysis. Those angles are measured preoperatively on an AP radiograph - the angle of Gekeler and on a profile one - the PTA.

Results: The results were estimated statistically by means of Paired Samples Test (a), Chi-square tests and test statistics.The results were classified using the clinical classification of Heyman and Herdon, 1945, as well as the radiographic one of Boyer et al. 1981. For an average period of follow-up $9.92 \pm 3.93$ years we estimate, according to Heyman and Herdon classification, 19 excellent, 17 good, 5 fair and 1 poor clinical result. According to the radiographic classification of Boyer et al., we estimate 31 cases as zero grade, 10 cases as grade I and one as grade III result. There is only one complication in our series-avascular necrosis and three intraoperative trochanteric fractures, all with good final results.

Conclusions: In our opinion, the presented operative technique is the easiest type of intertrochanteric osteotomy for correction of chronic cases of SCFE.

Significance: The obtained results are comparable with a control group treated by Imhäuser-Southwick operation.

9

\section{TREATMENT METHOD AND ITS COMPLICATION IN SCFE}

\author{
M. Kamegaya, C. Mannoji, J. Nakamura, K. Takahashi, \\ M. Takazawa, T. Kenmoku, T. Saisu
}

Purpose: We investigated the relationship between treatment methods and the occurrence of complications (AVN or chondrolysis) in SCFE patients who have been treated with various surgical methods from 1985 to 2005.

Methods: One hundred and forty-seven patients (164 hips) were reviewed for this investigation. The average follow-up term was 6.5 years $(2-15)$. Seventeen patients were affected bilaterally, 113 patients were boys and 34 were girls. One hundred and eighteen hips were classified as stable type slips and 46 as unstable type slips. We divided the past 20 years into two periods due to treatment strategy. The first period was from 1985 to 1995 , when in-situ fixation (using two or three pins) was indicated if posterior slip angle (PSA) was $30^{\circ}$ or less, and various osteotomies such as Southwick osteotomy, simple flexion osteotomy and Dunn's osteotomy were indicated if it was more than 30 . The second period was from 1996 to 2005, when in-situ fixation (using single screw) was indicated if it was $40^{\circ}$ or less, and a Pre-Operative computed Tomography Assisting Flexion osteotomy (POTOF osteotomy, JPO-B 2004) was indicated if it was more than 40 . We created this type of osteotomy and have been performing it ever since. For unstable hips, manual reduction was carefully performed in the first period, but in the second period, gentle repositioning was performed if needed for adequate bone stock for internal fixation, and afterwards POTOF osteotomy was considered if patients complained of some difficulties. In each period, the occurrence of complications and their possible causes were analyzed.

Results: In the first period, 56 hips were treated with in-situ fixation, including 4 in post-manual reduction and 30 were treated with various osteotomies: 17 with Southwick osteotomies, 9 with simple flexion osteotomies and 4 with Dunn's osteotomies. AVN was found in 5 hips: 2 hips after manual reduction and 1 each after Southwick osteotomy, simple flexion osteotomy and Dunn's osteotomy. Chondrolysis was found in 7 hips: 4 hips after Southwick osteotomies and 3 after in-situ fixation. In the second period, 53 hips were treated with single screw fixation, including 5 post-manual reduction and 25 were treated with POTOF osteotomies. AVN was found in 3 hips after manual reduction. Chondrolysis was found in only one hip after POTOF osteotomy. No AVN was found after POTOF osteotomy.

Conclusions: Manual reduction aiming for perfect reduction in unstable type slips was the most dangerous method for AVN. Southwick osteotomies showed the possibility of chondrolysis and a little of AVN. Acute correction and excessive post-operative valgus were responsible for $\mathrm{AVN}$ in various osteotomies, except POTOF osteotomy.

Significance: Our present strategy is reasonably safe with single screw fixation and POTOF osteotomy for stable type slips and with gentle repositioning, followed by POTOF osteotomy if necessary, for unstable type slips. Manual reduction must be avoided.

\section{0}

\section{BOTULINUM TOXIN TREATMENT OF THE BICEPS BRACHII MUSCLE IN CHILDREN WITH CEREBRAL PALSY CAUSES MUSCLE TISSUE REGENERATION}

\author{
E.M. Pontén, S. Arnardottir, S. Gantelius
}

Purpose: Botulinum toxin is used for temporary chemical denervation of relatively over-active flexor muscles in children with cerebral palsy $(\mathrm{CP})$, with the aim of more balanced joints. This indication was initiated almost 10 years ago, and the popularity of the treatment is large and increasing. But, till now, no prospective studies have been made on the effect of botulinum toxin on spastic muscle tissue in humans.

Methods: During nitrous oxide anesthesia percutaneous muscle biopsies were taken from the biceps brachii muscle of children with unilateral spastic $\mathrm{CP}(n=6$, mean age 10 years, range $5-13)$. The biopsies were taken immediately before the first injection of botulinum toxin A (Botox ${ }^{\circledR}$, Allergan), and just before clinically needed reinjection at 3-6 months and 12-16 months. Cryosections were stained with Hemotoxyline-eosin for general morphology and ATPase stainings were made for fibre typing. Monoclonal antibodies were used to detect different myosin heavy chains, vimentin and N-CAM.

Results: Biopsies taken up to 16 months after a botulinum toxin injection had an increased variability of the fibre area. There was 
an increased expression of vimentin, a marker for regeneration. $\mathrm{N}$-CAM was increased in an abundance of newly formed fibers (activated satellite cells) and also in larger cells. There was a trend of more type 2 fibers at up to 6 months after an injection, but no difference in the ratio type 1 /type 2 fibers when the interval was 12-16 months.

Conclusions: Treatment with botulinum toxin induces regeneration in the spastic muscle, probably due to the temporary denervation. Even long after reinnervation is considered to have occurred, there are signs of regeneration in the muscle.

Significance: The benefits of botulinum toxin treatment of the upper limb in CP in children are not yet evidence based (Cochrane Rev). Regarding side effects, the treatment is safe (Naumann, Albanese et al. 2006). However, the long-term effect on the muscle tissue itself is important and remains to be determined.

\section{1}

\section{LONG-TERM USE OF BOTULINUM TOXIN TYPE A (BTX-A) IN CHILDREN WITH CEREBRAL PALSY: TREATMENT CONSISTENCY}

G. Molenaers, A. Van Campenhout, E. Ortibus, J. De Cat, K. Desloovere, P. De Cock, P. Pauwels, V. Schörkhuber

Purpose: The aim of the study was to evaluate the stability of dosage, treatment intervals and efficacy in long-term, multi-level, high-dosage treated children with cerebral palsy $(\mathrm{CP})$ and to study the evidence for safe and stable response to this treatment. It was hypothesized that (1) the mean total BTX-A dosage does not increase and (2) the mean intervals between treatments and (3) the efficacy do not decrease within the longitudinal follow-up.

Methods: This is a retrospective cohort study. Between 1996 and $2005,10 \%(N=106)$ of all patients treated with BTX-A $(N$ 1,020 ) at the multidisciplinary clinic for children with $\mathrm{CP}$ (referral centre), received at least 4 BTX-A treatment sessions. Information on diagnosis, age at treatment, dosage and target muscles was extracted for each patient and treatment session from the electronic patients files. Mean dosage, de/increase of dosage, treatment intervals for consecutive treatment sessions, and mean age at treatment sessions were evaluated for the whole treatment group and separated groups (di-, hemi-, quadriplegia). The Goal Attainment Scale (GAS) was defined for a subgroup of 38 patients, to evaluate the functional outcome of each treatment session. A mixed model procedure was used for repeated interval/ ratio data, taking diagnosis into account. Unpaired t-test was used to evaluate the difference between mean age at first treatment.

Results: Patients were followed 4.5 years on average (range 1.78.8 years) and received $4-12$ BTX-A treatment sessions. $79 \%$ of all treated patients had diplegia, 9\% had hemiplegia, and $12 \%$ quadriplegia. Patients were treated with a mean dosage of $23.5 \mathrm{U} / \mathrm{kg}$ bw $( \pm 5.2)$ at first treatment with stable subsequent values $(23.4 \pm 5.5$ at the second, $22.2 \pm 5.7$ at the third and $21.3 \pm 6.2$ at the fourth session) (NS, $P=0.1202$ ). The mean age at first treatment was 4.5 years, with a majority of patients $(76 \%)$ first treated within 2 and 4 years of age. Treatment intervals of 1.1 year $( \pm 0.7)$ remained stable within subsequent treatments. The majority of patients $(94 \%)$ received multilevel treatment. The strategy changed at the third and fourth treatment sessions, when multi-level treatment decreased in favour of targeted treatment including two proximal levels. The mean GAS T-score at each session was significantly higher than the expected mean of 50 , indicating a successful outcome.
Conclusions: Long-term (repeated), high-dosage, multi-level BTXA applications can be considered a safe, stable and successful treatment option for children with $\mathrm{CP}$, thereby indirectly indicating the non-development of antibody formation.

Significance: Additional information on long-term efficacy and safety of high-dosage, multi-level BTX-A is required in order to expand our current knowledge of BTX-A treatment in cerebral palsy.

\section{2}

\section{DISTAL RECTUS TRANSFER IN CHILDREN WITH CEREBRAL PALSY: DOES IMPROVEMENT PERSIST LONG TERM?}

\author{
F. Miller, A. Agarwalla, C. Church, D. Thawrani, K. Dabney, \\ N. Lennon, S. Marchesi
}

Purpose: Distal rectus transfer is commonly performed to improve knee flexion and toe drag in patients with spastic CP. This IRB approved retrospective cohort study examines long-term outcomes and aims to determine if pre-operative factors can predict success.

Methods: A gait analysis was performed on 54 patients (87 limbs; GMFCS score of I-IV) pre-operatively (mean age 7.35), postoperatively ( $<3$ years post), and long-term (mean 9.2 years after surgery). Multivariate statistical analysis was used to determine which pre-operative physical exam and gait parameters could predict outcome.

Results: Peak knee flexion in swing phase increased $6^{\circ}$ post-operatively, but returned to baseline levels at long-term follow-up $(P<0.001)$, while the range of knee flexion declined in the long term by $4.31^{\circ}(P<0.01)$. Pre-operative peak knee flexion was a predictor $\left(R^{2}=0.640, P<0.0001\right)$ for both change in peak and range of knee flexion post-operatively $(P<0.0001, r=-0.770$ and -0.449 respectively) and long-term $(P<0.0001, r=-0.680$ and $P=0.046, r=-0.304)$, while the Gross Motor Function Measure (GMFM) predicted timing of peak knee flexion in swing both long-term and short-term $(P<0.001, r=-0.53$ and -0.66 , respectively).

Conclusions: The regression of knee flexion in swing, despite its initial improvement, demonstrates the importance of long-term follow-up. Lower peak knee flexion in swing predicted greater long-term improvement, and a higher GMFM predicted a better long-term outcome. However, our analysis was able to detect only large effects, and we aim to increase our sample size to determine if other variables cause less-visible effects.

Significance: Increase in peak knee flexion in swing phase did not last long-term in patients with cerebral palsy who received a distal rectus transfer.

\section{3}

\section{TREATMENT OF STIFF KNEE GAIT IN CHILDREN WITH CEREBRAL PALSY}

A. Presedo, B. Ilharreborde, E. Morel, F. Fitoussi, G.F. Penneçot, M. Laassel, M. Mehrafshan, P. Souchet

Purpose: To evaluate the effectiveness of distal rectus femoris (RF) release versus transfer to treat gait abnormalities of the knee in ambulatory children with cerebral palsy. 
Methods: Ninety-three children were included in this study. Thirtytwo patients underwent RF transfer at a mean age of 11.8 years and 61 underwent distal RF release at a mean age of 12.5 years. Indications for surgery included RF contractures, abnormal RF activity during swing phase (EMG) and kinematic characteristics of stiffknee gait. All patients had pre- and post-operative $3 \mathrm{D}$ gait analysis and EMG at 1 year follow up. To evaluate outcomes, patients were grouped by pre-operative knee kinematics (swing-phase peak knee flexion (PKF) $<50^{\circ}$ or PKF $>50^{\circ}$ occurring later than $77 \%$ of the cycle). All data was analyzed statistically.

Results: For the group of patients with PKF $<50^{\circ}$, this value increased significantly after RF transfer $(P=0.005)$ and after RF release $(P=0.03)$. Children with PKF later than $77 \%$ of the cycle also showed significant improvement after both procedures $(P=0.001 ; P=0.02)$. All patients experienced a significant decrease of muscle contractures.

Conclusions: According to the results of this study, both RF transfer and release brought significant results. We opt for distal $\mathrm{RF}$ release, since is technically easier, particularly when one-stage multilevel procedures are being performed.

Significance: To get a better understanding of stiff knee gait treatment in children with $\mathrm{CP}$.

\section{4}

\section{ANTHROPOMETRIC MEASUREMENTS ON GROWTH OF AMBULANT CEREBRAL PALSY CHILDREN AND ADOLESCENTS}

D.G. Pasparakis, C.M. Nestoridis, M.E. Pentarakis, M.N. Tziomaki, N.G. Darras, N.M. Papandreou

Purpose: To examine the anthropometric features of ambulant CP children and adolescents in order to document skeletal growth deviations compared to a normal population.

Methods: There were 39 normal individuals, 83 diplegics and 31 quadriplegics (5-18 years of age) ambulant CP subjects. Physical growth percentile analysis of height and weight was conducted for CP subjects, using the standard NCHS norms. Descriptive statistics and ANOVAs were used to test for differences among groups. A cross-sectional study was contacted using data collected from ambulant CP subjects. Height and weight measurements were combined with detailed 3D photogrammetric measurements of all body segments. Head, Trunk, Arm, Forearm, Thigh and Shank were analyzed. Two different normalization methods were applied: normalization to body height and normalization to trunk length. Since CP subjects develop contractures influencing their standing height, normalization to the subject's trunk length was conducted. Results: Subject's characteristics (Mean \pm SD): Normal 39 (F33, M6), Age $26.7 \pm 7.6$, Height(m) $1.75 \pm 0.11$, Weight(kg) $73.6 \pm$ 13.1. Diplegics 83(F41, M42), Age $14.9 \pm 7.2$, Height(m) $1.47 \pm 0.18$, Weight $(\mathrm{kg}) 44.1 \pm 17.4$. Quadriplegics 30(F16,M14), Age $14.6 \pm 6.9$, Height $(\mathrm{m}) 1.43 \pm 0.19$, Weight $(\mathrm{kg}) 42.9 \pm 14.7 . \mathrm{CP}$ physical growth percentiles (Mean \pm SD): Diplegics 60 , Height $(m)$ $36.4 \pm 26.6$,Weight $(\mathrm{kg}) 46.9 \pm 33.6$. Quadriplegics 21 , Height $(\mathrm{m})$ $19.4 \pm 23$, Weight $(\mathrm{kg}) 38.7 \pm 28.9$. ANOVA analysis showed significant ratio differences: CP Head to Height $>\operatorname{Normal}(P \leq 0.001)$, CP Trunk to Height $>$ Normal $(P \leq 0.001)$, CP Thight o Height $<$ Normal $(P \leq 0.001)$, CP Shank to Height $<$ Normal $(P \leq 0.001), \mathrm{CP}$ Head toTrunk $>$ Normal $(P \leq 0.001), \mathrm{CP}$ Trunk toTrunk $>$ Normal $(P \leq 0.001), \mathrm{CP}$ Forearm to Trunk $>$ Normal $(P \leq 0.001), \mathrm{CP}$ Thigh toTrunk $<$ Normal $(P \leq 0.001)$, CP Shank toTrunk $<\operatorname{Normal}(P \leq 0.001)$. Non significant differences were found between Diplegic and Quadriplegic subjects in any of the segment ratios to Height or Trunk.
Conclusions: Mean height of ambulant CP individuals was found to be $17 \%$ decreased compared to NCHS norms but their weight $12 \%$ increased compared to the expected for their age and height values. Strong significant differences were found between Normal and CP subject's segment. Head and Trunk ratios are increased in $\mathrm{CP}$ subjects while Thigh and Shank ratios are decreased. These results indicated that cerebral palsy has a negative effect on growth of the lower limbs segments. Head, Trunk showed increased growth while upper limp segments did not show to be affected. Significance: The documentation of deviations of body segment proportions in ambulant CP children and adolescents can reveal the effect of cerebral palsy on body segments growth. It can also be documented weather these deviations are segment specific.

\section{RESULTS OF ORTHOPAEDIC SURGERY WHEN THE SURGEONS FOLLOW THE GAIT ANALYSIS RECOMMENDATIONS IN CHILDREN WITH CEREBRAL PALSY}

\section{T. Terjesen, B. Lofterød}

Purpose: During the last two decades it has become common to correct all abnormalities in one operation (multilevel surgery) in ambulant children with cerebral palsy. An essential condition is optimal preoperative evaluation and we use 3-dimensional gait analysis in that respect. The purpose of the present study was to assess the short term outcome in a setting where the orthopedic surgeons follow the recommendations from the preoperative gait analysis.

Methods: 55 ambulant children had been clinically examined for gait problems by orthopedic surgeons who proposed a surgical treatment plan including all the specific surgical procedures that were considered necessary. The children then underwent 3-dimensional gait analysis, which was evaluated by a multidisciplinary team (orthopedic surgeon, child neurologist, orthotist, and physiotherapist). Based on the gait analysis, treatment was recommended and the surgical procedures were specified. The orthopedic surgeons followed the recommendations from gait analysis in more than $90 \%$ of the procedures. All the operated children underwent gait analysis about 1 year postoperatively and the non-operated children had a follow-up analysis about 2 years after the initial one. Results: 47 children, aged 11 (5-19) years, underwent surgical treatment. Dependent on accordance between initial clinical examination, gait analysis, and subsequent surgery, three groups were analyzed. In group A there was agreement between clinical proposals, gait analysis recommendations, and subsequent surgery in 128 specific procedures. In group B no surgery had been proposed after clinical examination but 54 procedures were performed based on gait analysis. In group C 55 surgical procedures had been proposed after clinical examination but not performed because they had not been recommended by gait analysis. The kinematic results were satisfactory in all the three groups, with improvements in most of the kinematic gait parameters in children who had undergone surgery and no deterioration in children who were not operated.

Conclusions: Gait analysis was useful in confirming clinical indications for surgery, in defining indications for surgery that had not been clinically proposed, and for excluding surgery that had been clinically proposed.

Significance: We recommend 3-dimensional gait analysis in all ambulant children with cerebral palsy, provided that the recommendations from the analysis are followed by the orthopedic surgeons. 
16

\section{EFFECT OF ANKLE FOOT ORTHOSES ON 3D TRUNK AND PELVIC MOTION DURING GAIT IN CHILDREN WITH CP}

\author{
A. Van Campenhout, E. Ortibus, G. Molenaers, K. Desloovere, \\ P. Pauwels, P. Van de Walle
}

Purpose: The purpose of this study was to evaluate trunk and pelvic motion during barefoot gait and during walking with Ankle Foot Orthoses (AFOs), in children with CP. The specific objectives were two-fold: (1) to compare trunk motion of children with $\mathrm{CP}$, with age related normal controls, and (2) to evaluate the effect of AFOs on trunk motion in children with CP.

Methods: 30 children with CP (pathological group: 18 diplegia, 12 hemiplegia), as well as 51 age-related normal children (control group) were included in the study. All children received full-body $3 \mathrm{D}$ gait analysis in barefoot condition as well as with AFO's. This study mainly focused on the kinematics of trunk and pelvis (18 gait parameters). The AFOs were posterior leaf springs, applied bilaterally. In each condition, the mean and SD for all parameters were calculated for the total pathological and normal group, as well as separately for children with diplegia and hemiplegia. Further data analysis included three types of comparisons using Student's $t$-test: (1) CP with controls, (2) hemiplegia with diplegia, (3) barefoot walking with AFO walking.

Results: The averaged walking pattern of children with CP was characterized by increased ROM at the pelvis, spine and thorax, compared to normal controls. This pathological pattern is more pronounced for children with diplegia compared to hemiplegia, however, the asymmetry (pelvic and thoracic obliquity and retracted pelvis and protracted thorax) is increased in hemiplegics, compared to diplegics. Both, children with diplegia and hemiplegia, showed significantly increased frontal and transverse ROM at the spine and thorax $(P<0.0005)$ in AFO condition compared to barefoot condition. At the level of the pelvis, the transverse ROM significantly decreased in AFO condition compared to barefoot $(P<0.005)$.

Conclusions: Children with $\mathrm{CP}$ walked with abnormal orientation and increased ROM of trunk, compared to normal children, indicating for increased asymmetry and instability. Although AFO's reduced pelvic instability, trunk ROM significantly increased when walking with AFO's compared to barefoot. Increased trunk ROM with AFOs might be considered as a compensatory strategy.

Significance: Although pathological motor patterns at the level of the trunk are quite frequently observed in children with $\mathrm{CP}$, little attention has been focused on typical trunk motion during gait. More insight into trunk motion can help to recognize, define and evaluate optimal treatment strategies.

\section{7}

\section{CHARACTERISTICS OF CHILDREN WITH HIP DISPLACEMENT IN CEREBRAL PALSY}

\section{G. Hägglund, H. Lauge-Pedersen, P. Wagner}

Purpose: To analyze the characteristics of children with cerebral palsy (CP) who develop hip displacement, in order to optimize a hip surveillance program.
Methods: In a total population of children with CP in southern Sweden a clinical and radiological follow-up of the hips was carried out as a part of a hip prevention program started in 1994. The program includes a continuing standardized registration of passive joint motion, gross and fine motor function, clinical findings and treatment. The children are examined twice a year until the age of 6 years and then once a year. Measurement of passive joint of motion is done with a goniometer in stated and standardized positions and. The program also includes a standardized radiographic follow-up of the children's hips. The migration percentage (MP) is measured on all radiographs. The present study is based on 212 children born 1990-1997, followed until 9-16 years of age. Passive range of hip abduction, external rotation, internal rotation and extension was recorded from the examination performed at the same age as the first radiographic presentation of hip displacement. The Cox model was used for evaluating effects of range of hip motion on the risk of developing MP $>33$ or $>40 \%$.

Results: Of the 212 children, 38 (18\%) developed displacement with MP $>40 \%$ and further $19(9 \%)$ MP was between 33 and $39 \%$. Mean age at first registration of hip displacement was 4 years, but some hips showed MP $>40 \%$ already at 2 years of age. The passive range of hip motion at the time of first registration of hip displacement did not differ significantly from the findings in hips without displacement. The risk of hip displacement varied according to $\mathrm{CP}$-subtype, from $0 \%$ in children with pure ataxia to $79 \%$ in children with spastic tetraplegia. The risk of displacement (MP > 40\%) was directly related to the level of gross motor function, classified according to the gross motor function classification system, GMFCS, from $0 \%$ in children in GMFCS level I to $64 \%$ in GMFCS level V.

Conclusions: Hip displacement in CP often occurs already at 2 3 years of age. Range of motion is a poor indicator of hips at risk. Consequently radiographic follow-up is necessary. The risk of hip displacement varies according to both CP-subtype and GMFCS. It is sometimes not possible to determine subtype before 4 years of age, and at present several definitions and classification systems are used. GMFCS is valid and reliable from 2 years of age, and it is internationally accepted.

Significance: It is of the utmost importance that children with CP are identified early, and that children at risk are examined radiographically as early as possible. We recommend a hip surveillance program for children with $\mathrm{CP}$ with radiographic examinations based on the child's age and GMFCS level.

\section{8}

\section{ASSOCIATIONS BETWEEN ORTHOPAEDIC FINDINGS, AMBULATION AND HEALTH RELATED QUALITY OF LIFE IN CHILDREN WITH MYELOMENINGOCELE}

\author{
A. Danielsson, Å. Bartonek, E. Levey, H. Saraste, K. McHale, \\ P. Sponseller
}

Purpose: The aim of orthopedic treatment in patients with myelomeningocele is to minimize contractures and joint dislocations, thereby favoring ambulation. Better ambulatory function is thought to improve the management of activities of daily living. The focus for this study was to evaluate ambulatory function and health related quality of life (HRQoL) in children with myelomeningocele.

Methods: Thirty-eight patients (aged 4-16 years) with neurological deficit from myelomeningocele were examined in an unbiased follow-up. This included a physical examination using validated 
methods for ambulatory function and neuromuscular status, chart reviews and evaluation of radiographs in terms of hip dislocation and spine deformity. The Pediatric Evaluation of Disability Inventory (PEDI) was used to measure mobility, self-care and social function, and the Child Health Questionnaire (CHQ-PF50) for measuring HRQoL.

Results: $63 \%$ of the patients had quadriceps strength of 4-5 and $26 \%$ had spasticity around hip and/or knee joint muscles. Onefourth of the patients had some degree of mental retardation. Nineteen children were ambulatory and 19 non-ambulatory. Muscle function class, quadriceps strength, spasticity in hip and/ or knee joint muscles, hip dislocation, hip flexion contracture $>20^{\circ}$ or spinal deformity with Cobb angle above $30^{\circ}$ each significantly affected functional mobility/PEDI. Self-care/PEDI was significantly impaired by hip flexion contracture $>20^{\circ}$ and absence of functional ambulation. General health related quality of life was significantly lower in this patient group than for US norms. Both the Physical component summary score $(\mathrm{PhS})$ and the Psychosocial component summary score (PsS) of the CHQPF50 were significantly lower than age-matched norms, 31,5 vs. 53,0 $(P<0.0001)$ and 47,9 vs. 51,2 $(P=0.036)$ resp. Functional physical abilities were shown to be of importance for achieving a better quality of life; being ambulatory, having better functional mobility, self-care or social ability and being without learning disabilities each improved physical quality of life.

Conclusions: Children with myelomeningocele had significantly reduced quality of life compared to healthy individuals. Patients with hip dislocation, hip flexion contracture and/or those who were non-ambulatory had reduced functional mobility and/or selfcare, which correlated with a lower Physical Functioning subscale of the CHQ. These findings strongly support efforts to reduce hip flexion contracture, hip dislocation and scoliosis and to aim for getting patients ambulatory.

Significance: Children with myelomeningocele have reduced quality of life. Further impairment of physical function and quality of life might be prevented by treatment against hip dislocation, significant hip flexion contracture or scoliosis.

\section{9}

\section{ORTHOPEDIC MANIFESTATIONS OF CONGENITAL MYOTONIC DYSTROPHY DURING CHILDHOOD AND ADOLESCENCE}

\section{F. Canavese, M. D. Sussman}

Purpose: Congenital Myotonic Dystrophy (CMD) is a dominantly inherited disorder manifested in childhood by muscle weakness which can be profound at birth, but which progressively improves over the first few years. CMD represents the severe end of the spectrum of Myotonic Dystrophy, which in milder cases may not be diagnosed until adulthood. The goal of the study was to identify and quantitate the musculoskeletal deformities which may significantly affect the function of children with CMD.

Methods: A retrospective chart and radiograph review was performed after Institutional Review Board approval for all cases of myotonic dystrophy from 1987 to 2004 followed at a children's specialty orthopaedic hospital. Inclusion criteria were either a conclusive testing for CMD by gene testing, electromyography and/or muscle biopsy in the child or parent and the presence of a typical clinical picture. Skeletal manifestations were classified by body segment (upper extremity, hand, spine, hip, lower extremity, foot) and by the type of deformity. Surgical procedures and outcomes were also documented.
Results: 30 children and adolescents met the inclusion criteria. The male/female ratio was 1 ( 15 boys and 15 girls). In 27 cases the mother and in 2 cases the father transmitted the disease; in one case was impossible to reconstitute the family history of the child, who was adopted. The mean age at onset of gait was 29 months. The mean follow-up was 11.4 years (range: 3-20 years). 22 out of $30(73 \%)$ children underwent surgery for lower extremity, foot or spinal related deformities. No contractures or deformities were observed in the upper extremities. Spinal deformities affected 9 patients $(30 \%)$, and 3 of these required surgery. These spinal deformities when present usually had an early onset and included scoliosis as well as kyphoscoliosis. Problems at the level of the hips and knee were infrequent and included only two patients who had unilateral hip abduction contracture, and one patient who had significant bilateral fixed knee flexion contracture. Congenital clubfoot occurred in $17 \%$, and generally responded well following posteromedial release and recurrence occurred in only one case. Developmental equinus and equinovarus exclusive of clubfoot affected seven $(23 \%)$ patients, $70 \%$ of whom required surgery. Outcome following Achilles tendon lengthening was positive, and many of the children began walking soon after the Achilles lengthening, and recurrence did not occur.

Conclusions: Child with CMD is at high risk for musculoskeletal deformities of the spine and lower extremities. In our experience correction and improved function was likely following surgery.

\section{0}

\section{SLOTTED ACETABULAR AUGMENTATION (SAA) FOR TREATMENT OF NEUROMUSCULAR HIP DYSPLASIA IN CHILDREN AND ADOLESCENTS}

\section{Lugmair, C. Vuillerot, F. Chotel, F. Sailhan, J. Berard, V. Cunin}

Purpose: The reported prevalence of subluxation and dislocation of the hip in spastic cerebral palsy is very hight. The treatment goals of the neuromuscular hip is to re-establish a well-contained stable joint, to prevent further displacement of the non dislocated hip, to relieve pain, and to facilitate daily care, sitting posture and transferring.

Methods: During a 20-year period, we carried out the slotted acetabular augmentation (SAA) procedure, alone or as part of a combined one-stage approach, consisting of soft tissue lengthening and/or a proximal femoral osteotomy, for the treatment of 39 dysplastic hips in 36 patients with an underlying neurological disorder. The mean age at the time of operation was 9 years 7 months (range 6 years 10 months to 14 years 7 months) in the 12 patients (15 hips) with an open triradiate cartilage and 14 years 6 months (range 11 years 7 months to 19 years 11 months) in the 24 patients (24 hips) who were skeletally mature. Seven hips had flaccid paralysis and 32 were spastic. In 22 hips, preoperative radiographs showed major aspherical femoral head deformities. All had subluxation or dislocation of the hip and severe acetabular dysplasia. In a patient questionnaire, patients or parents/caregivers were further asked to assess the patient's preoperative and postoperative status. All patients were followed postoperatively by serial radiographs to assess specific measurements.

Results: Before operation the mean migration index was $62 \pm 18$ per cent, the mean acetabular index was $32 \pm 5^{\circ}\left(51 \pm 4^{\circ}\right.$ Sharp angle), and the mean centre-edge angle was $-5 \pm 13^{\circ}$. On immediate postoperative radiographs, they were $3 \pm 5$ per cent, $12 \pm 7^{\circ}$ (35 $\pm 5^{\circ}$ Sharp angle), and $43 \pm 11^{\circ}$. At final follow up, the 
average migration index was $13 \pm 14$ per cent, the mean acetabular index was $15 \pm 6^{\circ}\left(35 \pm 6^{\circ}\right.$ Sharp angle), and the mean centreedge angle was $40 \pm 12^{\circ}$. Nineteen hips required soft tissue lengthening and 18 hips had a proximal femoral osteotomy at the time of the shelf procedure. At the latest follow-up (mean duration, 6 years 7 months), 35 of the 39 hips remained anatomically reduced. Pain was eliminated in 18 and reduced in 2 of 20 patients who had preoperative pain. A review of the literature is given to assess efficacy and determine the role of SAA for treatment of neuromuscular hip dysplasia in comparison with other treatment options.

Conclusions: Our results suggest that SAA is a successful salvage procedure to treat neurogenic acetabular dysplasia with or without femoral head deformities in skeletally immature and mature patients.

Significance: We still recommend even in young patients, this straightforward technically safe and simple procedure, especially in case of major hip dysplasia with femoral head deformity.

\section{1}

\section{OPERATIVE TREATMENT OF THE UPPER EXTREMITY IN OSTEOGENESIS IMPERFECTA}

\section{Rogalski, J. Zacher, M. Deja}

Purpose: There are only little information about upper extremity dysfunction and indications for operative intervention in OI. Deformities of the upper extremity in OI are seen in nearly $40 \%$ of the patients. Greater deformities can compromise independence in daily life and mobility. Fractures of the upper extremity in OIC were found in more than $60 \%$. We analyzed, in which cases there was a need for an operative intervention.

Methods: Retrospective analysis of 13 of $33(19 \%)$ cases of OI, who showed clinical relevant deformities and problems of upper extremities. 10 of them have undergone operative treatment for the following indications: fracture, deformities and pseudarthrosis. The preoperative numbers of fractures per year were: 4 [1-13], the median age at 1st op.: 8.5 [6-17] years. The humerus was affected 16 times. The main localization was the distal third and main direction anteromedial (mean: $\left.54^{\circ}[15-80]\right)$. In 6 of 13 patients $(46 \%)$ the ulna was affected, bilaterally in 3 . The main ulnar deformity localization was the middle third and main direction voloulnar (mean: $32^{\circ}[15-$ $\left.\left.112^{\circ}\right]\right)$. The radius was affected in $6 / 13$ Patients $(46 \%)$, bilaterally in 3 . The main radial localization was the middle third and main direction: voloulnar (mean: $26^{\circ}\left[10-89^{\circ}\right]$ ). We performed 19 operations (humerus: 10, ulna: 7 and radius: 2). Osteosynthesis was done with Rush pins, K-wires, ESIN and cerclage (3 olecranon fractures). The postoperative treatment consists of initial short time immobilization and early functional treatment.

Results: We found an adequate consolidation of fractures and corrective osteotomies. Complications were seen in 4/19 $(21 \%)$ : $1 \times$ Pin-Penetration, $1 \times$ Infektion, $1 \times$ temporary radial nerve paresis and $1 \times$ recurrent fracture with dislocation. For the evaluation of function pre and post intervention, we used the Upper extremity Scale of Pediatric Outcomes Data Collection Instrument (PODCI). There was found a slight increase from 39 to 62 .

Conclusions: In comparison with lower extremity surgery in OIC operative interventions at the upper extremity are rarely indicated, mainly in Type III-cases. Operative fracture treatment allows an early restoration of function. A corrective osteotomy can be considered in case of affected function with a severe deformity. A good function with spontaneous correction of axial deviation can be achieved under conservative therapy with biphosphonate in hyperthrophic pseudoarthrosis. An atrophic pseudoarthrosis should be treated with spongiosaplasty and adequate retention. With the stabilizing effect of biphosphonates in OI patients we expect a further decreasing rate of necessary interventions. On the other hand can a stable bone offer new options for interventions like callus distraction and stepwise deformity correction also in patients with OIC.

\section{2}

\section{HOFFER TRANSFER MAY NOT ACHIEVE FUNCTIONAL SHOULDER ABDUCTION AND ELEVATION IN CHILDREN WITH ERB'S PALSY}

\section{Ghanem, F. Dagher, K. Kharrat, N. Choucair}

Purpose: To evaluate the risks and benefits of latissimus dorsi and teres major transfer to the rotator cuff in obstetrical brachial plexus palsy.

Methods: A retrospective review was conducted on 40 consecutive children with Erb's palsy who underwent a Hoffer procedure to improve shoulder abduction and external rotation. Mean age at surgery was 7.5 years (2-19.5 years). Active preoperative abduction was $69^{\circ}\left(20-90^{\circ}\right)$. The absolute gain in abduction and the achievement of functional active abduction-external rotation ( $>90^{\circ}$ active abduction, modified Mallet score) as well as loss in internal rotation (personal score) were recorded three and six months following surgery and at an average follow-up of 2.5 years (range 1-6 years). Age at surgery, severity of initial neurological deficit, preoperative active abduction angle and type of surgery were correlated to the end result. Patient (and/or parent) satisfaction was assessed.

Results: Active and passive external rotation improved in all patients. Active external rotation was good compared to the healthy side ( $<20^{\circ}$ difference) in $70 \%$ of patients. At last follow-up, the average absolute gain in abduction was $47^{\circ}\left(10-110^{\circ}\right)$ and $85 \%$ of the children presented functional abduction $>90^{\circ}$. The modified Mallet score (0-5) improved from 1.1 preoperatively to 2 three months postoperatively, four 6 months postoperatively and 4.3 at last follow-up. At final assessment, $51 \%$ of patients were able to bring their hand to the sacrum $(45 \%)$ or behind the back $(6 \%)$ without swinging. Parents were satisfied in $82 \%$ of cases. The absolute gain in abduction was independent from the severity of initial neurological deficit, preoperative angle of active abduction, and type of surgery. The chances of achieving final functional abduction $\left(>90^{\circ}\right)$ was 17 times greater when the preoperative abduction angle was $>60^{\circ}$. Mean age at surgery was 11 years for patients with poor final outcome for active abduction compared with 7 years for patients with good outcome.

Conclusions: Hoffer transfer is an effective procedure to improve shoulder abduction and external rotation. However, the achievement of a good functional result seems to be highly dependent on the initial angle of active abduction $\left(>60^{\circ}\right)$ and young age (3-8 years) at surgery. The greatest amount of functional improvement seems to be achieved during the first 6 months following surgery, an important issue for postoperative physical therapy. The major risk of this procedure remains the subsequent decrease in internal rotation which may significantly interfere with the simplest daily habits and/or activities.

Significance: Hoffer transfer for Erb's palsy achieves functional shoulder abduction and elevation in 3-8 year-old children with initial active abduction $>60^{\circ}$. 
23.

\section{PREDICTION OF LIMB LENGTH DISCREPANCY DURING ANTENATAL LIFE. A RADIOGRAPHICAL STUDY}

\author{
P. Kelly, A. Couture, A. Diméglio
}

Purpose: Lower limb buds appear during the second week of embryonic life and are well differentiated by the end of the 8th week. Primary ossification centres of femur and tibia appear by the end of the 8 th week and by 12 weeks the cartilaginous anlage is complete. By 14 weeks primary ossification is sufficient to allow accurate ultrasonographic measurement of femoral diaphyseal length. There are many established database for estimating fetal femoral length ultrasonagraphically. There is little data however on radiographical measurements of fetal femoral lengths. The aim of our study was to introduce radiographically measured fetal femoral lengths in order to improve our understanding of normal fetal femoral growth.

Methods: A group of 60 post-mortem fetal radiographs ranging from 14 to 39 weeks gestation were retrieved from our radiology department having excluded all cases with associated lower limb deformation. Femoral lengths were measured and plotted against gestational age. A standard growth curve was constructed and compared to the currently available data on ultrasonographic measurements. A growth velocity chart, growth remaining and an antenatal multiplier chart was also constructed.

Results: At 14 weeks the length of the femoral diaphyseal length was $14 \mathrm{~mm}$ and the tibia $11 \mathrm{~mm}$. At birth the femoral diaphyseal length was $75 \mathrm{~mm}$ and the tibia $62 \mathrm{~mm}$. The femoral diaphysis is $80 \%$ of total femoral length. Using the standard growth curve there was a strong correlation between our radiological measurements and previously published ultrasonographic measurements $\left(\mathrm{R}^{2}=0.9492\right)$. The femoral growth velocity curve demonstrated a rapid growth acceleration phase peaking at $12 \mathrm{~cm} /$ annum at 16 weeks, followed by a rapid deceleration phase reducing to a growth rate of $5 \mathrm{~cm} /$ annum at birth. The growth remaining was calculated for each week. At 24 weeks the growth achieved is $10 \%$ thus giving a multiplying factor of 10 . At birth the growth achieved is $20 \%$ giving a multiplying factor of 5 .

Conclusions: Previous studies on fetal femoral length have used ultrasound. This study verifies that radiographic measurement correlate with ultrasound measurements. We have further established a growth velocity curve for fetal femoral growth in order to further deepen our understanding of intra-uterine femoral growth. Significance: The understanding of normal intra-uterine growth is essential knowledge of a pediatric orthopedic surgeon when advising on antenatally detected deformities. This study allows us to make predictions throughout fetal life in relation to estimated limb length discrepancies.

\section{4}

\section{FEMORAL LENGTHENING WITH THE ALBIZZIA NAIL:} OUR EXPERIENCE

\section{Peña, J. Monasterio, L. Arana, O. Espinazo, R. Mongil}

Purpose: The purpose of this study is to evaluate the results of intramedullary nailing for femoral lengthening using the Albizzia Nail during a 10-year period (January 97-June 07).
Methods: Current methods of treatment for leg length discrepancy are based on distraction osteogenesis. This involves creating callus during gradual distraction using an external fixator or an intramedullary lengthening nail. We have reviewed the patients treated with the Albizzia femoral nail, during the period 1997-2007. We carried out femoral lengthening in 58 cases: bilateral in 22 patients, with 43 nails and unilateral in 15 patients.

Results: We have indicated limb lengthening for 2 types of conditions:

1. Inequalities in length of the lower limb, regardless of cause.

2. Constitutional short stature.

The operated patients ranged in age from 13 to 38 years, with an average age at the moment of surgery of 16 years. All the lengthened femurs healed without bone union complications or infections. We have not seen secondary bone axis deviation. On the other hand, we have had quite a few intraoperative complications, mainly in the very first cases.

Conclusions: In our experience, comparing with the External Fixators, the intramedullary nail has a few adventages: Decreased risk of infections, skin incisions are small with better cosmetic results, no secondary bone axis deviation, no osteoporosis, easier postoperative physiotherapy and rehabilitation. Nail lengthening requires a surgeon skilled and experienced, with a full understanding of the procedure and a difficult learning curve.

Significance: The paper presents the currently available possibilities for using an intramedullary nail in femoral lengthening.

\section{5}

\section{CONGENITAL AND ACQUIRED DEFORMITIES IN CHILDREN CORRECTED WITH THE TAYLOR SPATIAL FRAME}

\section{J. Horn, H. Steen, L. P. Kristiansen}

Purpose: The Taylor Spatial Frame is a circular external fixator, where the rings are attached to each other by 6 telescopic struts, creating a hexapod. Varying the strut lengths changes the orientation of the rings with respect to one another and can thus correct uniplanar or multiplanar deformities of length, angulations, translation or rotation. There are only few reports in literature about the use of the TSF in children. The aim of our retrospective investigation was to present and evaluate the use of the TSF in congenital and acquired deformities in children.

Methods: From October 2000 to August 2006, we operated 42 frames ( 19 femora, 23 tibiae) in 36 children ( 20 males, 16 females, mean age: 13, 5-16). Follow up after frame removal was at least 1 year. $30 \mathrm{TSF}$ were applied for congenital (Group I) and 12 for acquired deformities (Group II). Two patients were corrected for axial deformities without lengthening; one patient underwent femoral lengthening without any axial correction, while all other patients had complex deformities including axial defects and length differences (mean $37 \mathrm{~mm}$, range 7-80). Clinical and radiographic evaluation of all deformity parameters were done before surgery. Frames with specific strut lengths were built preoperatively according to deformity parameters, limb size and the planned mounting parameters. Deformity correction was done in either "chronic mode" (32) or in "total residual mode" (10), when mounting parameters had to be adjusted after surgery.

Results: Mean follow up after frame removal was 25 month (1272). All deformity parameters were corrected and the frames removed after bony consolidation. In 11 cases 1 or 2 additional "total residual" adjustments were necessary for fully correction of the deformities. Lengthening index was 2.3 months/cm in Group I 
and $1.9 \mathrm{month} / \mathrm{cm}$ in Group II. One patient in Group I received autologous bone transplantation to provide consolidation. Three patients in Group I sustained a fracture in the callotasis zone after frame removal, whereof two healed after internal and one after external fixation, and 2 patients had to be operated for extension contracture in the knee after femoral lengthening. No fractures or other major complications were observed Group II. Minor complications like superficial skin infections were observed in both groups.

Conclusions: The TSF is an excellent tool for the correction of complex deformities in children. The "total residual" mode allows precise correction of residual deformities. In our retrospective investigation, children with congenital deformities showed somewhat slower healing and a higher rate of complications compared to children with acquired deformities.

\section{6}

\section{PROXIMAL FEMORAL OSTEOTOMY IN PAEDIATRIC PATIENTS USING MONOAXIAL EXTERNAL FIXATION}

\section{R. Schiavon, A. Borgo, A. Micaglio, A. Rebeccato, S. Santini}

Purpose: Many orthopedic diseases and many disorders affecting the central and the peripheral nervous system may produce hip deformity and/or hip instability. When hip presents angular or rotational deformity of the femur, proximal femoral osteotomy could be needed. Osteotomy could be performed separately or in association to other surgical procedures. Plate and screws are commonly used to achieve stabilization of the osteotomy. Our purpose is to stress the possibility of using the external fixation to stabilize that osteotomy, focusing on advantages and problems of the technique.

Methods: We report 43 femoral osteotomies (34 patients) of proximal femur performed with monoaxial external fixation, 16 males and 18 females, mean age 8 years (from 3 to 11 years). In most of our cases, hip deformity were due to neurological diseases (23 ostetomies in cerebral palsy, 12 in spina bifida, 1 in posttraumatic paraplegia); we also had 4 operations in Perthes disease, 1 in Down syndrome, 2 in congenital hip dysplasia. Femoral osteotomy was performed alone in 24 cases, in 19 cases additional surgical procedures were performed (soft tissues surgery and/or pelvic osteotomy). Surgical approach for osteotomy and stabilization with external fixator is presented. All patients were followed up clinically and radiographically for an average time of 10 years.

Results: Correction of angular and torsional deformity was obtained in all patients. The average time for osteotomy healing and fixator removal was 3 months. Complications were: femoral fractures ( 1 case), delayed unions ( 2 cases), relapse of the deformity (3 cases), deep venous thrombosis (1 case), decubitus ulcer( 1 case), pin irritations (10 cases), pin osteolysis ( 5 cases).

Conclusions: Proximal femoral osteotomy accomplished by using the external fixation permits correction of the deformity with a mini-open surgical approach, surgical time of intervention is decreased, blood loss is minimized and no additional open surgery is needed for removing the fixator. That technique is useful when additional surgical procedures ad approaches are needed, locally or in more than one anatomical site (that is true especially in neurological child). On the other hand, it has to be declared that the learning curve is long, surgeons exposure to fluoroscopy is needed, nursing at home in necessary and several clinical and radiographic controls are carried out to focus on possible complications. Last but not least, external fixation could be not easy to accept by the child.

Significance: External fixation could be an effectual alternative to common surgical procedures to correct deformities of the proximal femur. In our opinion the technique is useful when surgical treatment with many approaches, i. e. during one-stage multilevel operation, is request. That is true especially in neurological patients, whose life is characterized by several surgical stages, both orthopedic and not.

\section{7}

\section{CORRECTIVE OSTEOTOMIES AND BONE LENGTHENING IN X-LINKED VITAMIN D RESISTANT HYPOPHOSPHATEMIC RICKETS (VDXLR)}

\section{E. Ramseier, G. Exner, S. F. Fucentese, T. J. Neuhaus}

Purpose: Vitamin D resistant hypophosphatemic rickets (VDXLR) is a metabolic disorder first described by Albright in 1937. Therapy consists of oral phosphate substitution and supplementation of active vitamin D compounds. Even in children with good compliance, there is a risk of developing rickets leading to bone deformities, pain and small stature. Corrections of bone deformities and lengthening procedures may be indicated for prevention of secondary problems as pain and degenerative arthropathy or cosmetic reasons. Problems related to surgical corrections include bone healing, need for 3-dimensional level procedure, type of osteosynthesis and the question of timing. A team-work approach is fundamental for successful treatment of patients with VDXLR.

Methods: 12 patients (3 males) with VDXLR were treated in our institution: 8 underwent surgical correction, 3 of them in combination with bone lengthening. In 3 patients the corrections were performed at the end of growth. Repeated osteotomies and lengthening were performed in 2 patients. Clinical endpoints were height, leg axis, and pain.

Results: The age of diagnosis of VDXLR ranged from birth to 11 10/12 year (median $39 / 12$ ). The median follow-up period was $78 / 12$ year (range 19/12-30). The age at last follow-up ranged from 2 to 30 year (median $136 / 12$ ). 8 of the 12 patients underwent surgery. 6 had a single bilateral surgical correction, while 2 had 3, respectively, 5 corrections. The age at first corrective intervention ranged from $35 / 12$ to 19 9/12 year (median 7 9/12). 3 patients underwent bone lengthening using an external fixator system. The operated patients were followed for a median period of $96 / 12$ year (range 3 10/12-30). The median follow-up from the last surgical intervention to the most recent consultation was $24 / 12$ years (range $13 / 12$ to $175 / 12$ ). At latest followup, 5 patients had a mature skeleton, and 7 including 4 treated surgically, were still growing. Indication for surgery was deformity in all operated cases, and in one patient also pain. No bone healing problems occurred. At the last follow up, 6 of 8 operated patients had physiological axis. 2 patients had mild genu vara, 1 complained of intermittent pain over the last $2 \mathrm{y}$. There was no radiological evidence of degenerative arthropathy.

Conclusions: In children with VDXLR, drug substitution remains the main pillar of therapy. In case of bone deformity, surgery can be safely performed, independently of bone maturation. The role of lengthening procedures to improve short stature needs further studies. Close cooperation between different disciplines and an individual approach for each single case is mandatory for optimal care and a high patient satisfaction. 


\section{8}

\section{LENGTHENING AND AXIAL CORRECTION OF LOWER LIMB IN OLLIER DISEASE}

\author{
P. Koczewski, A. Koch, M. Jozwiak, M. Shadi
}

Purpose: To evaluate the results of Ollier disease patients treated with Ilizarov method.

Methods: Material There were 10 patients ( 7 male, 3 female) at the age of 7 to 20 years (mean 12,8) in which 21 segments (10 femur, 11 tibia) were treated between 1996-2006. The indication for surgery in 19 segments was shortening ranged from 4 to $9 \mathrm{~cm}$ (mean 5,5), in 17 cases associated with axial deviation (varus 8, valgus 8 , procurvatio 2 ) in other 2 isolated axial deviation. Mean value of axial deviation was $18^{\circ}$ (from 10 to 35 ). In two cases short stature treatment was additional indication for surgery. Follow up ranged from 6 months to 10 years (mean 4 years 8 months). Ilizarov frame was used in 18 segments and monolateral EBI frame in other 3 . In 3 patients additional supramalleolar osteotomy for axial correction was done.

Results: Lengthening achieved ranged from 4 to $9 \mathrm{~cm}$ (mean 5.6). Treatment time (in frame) ranged from 4 to 10 months (mean 6.8) and lengthening index ranged from 1 to 1.6 months $/ \mathrm{cm}$ (mean 1.3). In all patients complete axial correction was achieved. Recurrence of the deformity was observed in most of the patients with active growth plates (4/6). Typical for external fixation methods superficial infection were observed while there were no major complications in analyzed material.

Conclusions: Ilizarov method in Ollier disease is safe and effective management of axial deviation and shortening. It allows correcting multilevel deformity correction in single procedure. In comparison to other etiologies lengthening index in Ollier patients is proportionally low. Axial correction is most often indication for surgery and needs multistage treatment during growth.

\section{9}

\section{CHANGES IN PAEDIATRIC TRAUMA CARE, A PROSPECTIVE EPIDEMIOLOGICAL STUDY}

\author{
N. Francis, A. Diméglio, P. Kelly
}

Purpose: In 1995 at the EPOS Symposium in Porto, Prof Mesquita Montes presented a proforma for pediatric fractures in order to establish a multicentre study on the epidemiology of children's fractures. This standardized proforma consists of 39 sections with 200 items.

Methods: From 1997-2007 we completed this proforma prospectively for each child presenting to our Emergency Department with a fracture. The data was collected and analysed on 11,899 fractures in 10,923 children in order to establish the epidemiology and change in management practices in our unit over the 10 year period.

Results: Our study correlated with previously reported studies in the literature. There was a predominance of upper limb fractures. The most frequent fracture overall was distal radial $(28 \%)$, hand $(13 \%)$, elbow $(12 \%)$ and forearm $(11 \%)$. The most frequent circumstances of the injury depended upon the age group. Under 2 years $91 \%$ were domestic, $<6$ years $55 \%$ were domestic accidents, $6-11$ years $33 \%$ occurred during outdoor play, $>11$ years $45 \%$ were sports related. There were 56 multiple traumas
$(0.005 \%)$. Epiphyseal fractures accounted for $18 \%$ overall but showed a pubertal peak. There were 27\% Salter I, 53\% Salter 2, 7.7\% Salter 3, 9\% Salter 4 and less than 0.5\% Salter 5, SalterRang and Peterson fractures. In terms of treatment we found a marked evolution during the 10 year period. The hospital admission rate reduced from $67 \%$ in 1997 to $32 \%$ in 2007 . The average length of stay for a femoral fracture reduced from 23 to 3 days. In terms of treatment, $65 \%$ required simple immobilization only, $23 \%$ required closed reduction and immobilization and $12 \%$ osteosynthesis (of which $55 \%$ were percutaneous). In relation to closed reduction, the number of patient required general anesthesia reduced from 50 to $14 \%$ over the 10 year period greatly reducing hospital admission rate. $30 \%$ of fractures were benign requiring no follow-up appointment.

Conclusions: Our unit serves a population of 3 million giving a pediatric population of 700,000 . Only $10 \%$ children are treated in a pediatric orthopedic unit with $90 \%$ being treated by adult orthopedic surgeons. The efficiency of a unit can be increased by identifying benign fractures reducing out-patient visits by $30 \%$, performing entonox reduction of appropriate fractures in the emergency room reducing the operative load by $56 \%$. Displaced supracondylar fractures, epiphyseal fractures and multiple traumas may be inappropriately treated in peripheral units and should be referred early to a specialised paediatric trauma unit.

Significance: This study has allowed us to optimize regional pediatric trauma care in collaboration with our adult orthopaedic colleagues, our administrators and our politicians. Significant reductions in hospital admissions, the rate of children needing general anaesthesia have allowed us to greatly increase the efficiency of our unit.

\section{0}

\section{TREATMENT OF RADIAL NECK FRACTURES IN CHILDREN ACCORDING TO MÉTAIZEAU}

\section{S. Endele, F. Fernandez, O. Eberhardt, T. Wirth}

Purpose: The treatment of dislocated radial neck fractures is difficult and therefore an arthrotomy of the elbow is often necessary. The intramedullary pinning according to Métaizeau allows closed reduction of dislocated radial neck fractures. The efficiency as well as the advantages and disadvantages of the technique according to Métaizeau are discussed in this clinical and radiological study.

Methods: 63 children with dislocated radial neck fractures were treated according to Métaizeau in the Orthopedic Department of the Olgahospital Stuttgart, Germany between 1993 and 2006. 55 children $(87 \%)$ with a mean age of 8 years were followed up. The average follow up time was 4 years. The radiological dislocation according to Judet, associated injuries of the elbow, the necessity of an arthrotomy and the occurred complications were documented. Range of movement, carrying ankle, pain and contentment were clinically followed up. Range of movement was classified under the elbow score according to Morrey et al. as well as the functional score according to Métaizeau et al. The results of the different dislocation types according to Judet were compared. Results: All Judet II (12), III (28) and 8 of 15 Judet IV fractures could be treated according to Métaizeau with closed reduction. $98 \%$ showed an excellent or good result according to Morrey et al., $90 \%$ to Métaizeau et al., two children had a fair and two a poor one $(10 \%)$. Reason for the failure was most often a limitation in pro-/supination. One had developed a synostosis, one a radial neck necrosis, one a Judet II fracture with complete translocation of the radial head. For one patient the reason for 
failure could not be found. The parents of the 13 children who could not be followed up clinically announced full range of movement and no restrictions in daily life matters. $40 \%$ of the patients had associated injuries (luxations of the elbow, ulnar/ radial epicondyles fractures, fracture of the olecranon or Monteggia like fractures).

Conclusions: All children with dislocated radial neck fractures but one had no restrictions in daily life matters and had no complains after being treated according to Métaizeau. The intramedullary pinning according to Métaizeau proved as a sufficient therapy for dislocated radial neck fractures being a minimal invasive technique. The technique allows an anatomical reconstruction and stable treatment of the fractures without arthrotomy and further soft tissue damage and therefore reduces severe complication as necrosis of the radial head or synothosis with consecutive limitation of movement.

\section{1}

\section{PHYSEAL FRACTURES OF THE DISTAL RADIUS AND ULNA: LONG-TERM PROGNOSIS}

\section{F. Mancini, E. Ippolito, F. De Maio, V. Potenza}

Purpose: The long-term prognosis of injuries to the distal physis of forearm bones, including complications such as radioulnar length discrepancy and styloid nonunion, has not been extensively studied. Radiographic prognostic criteria to predict physeal disturbance at trauma are also lacking. The aim of this study is to investigate both issues.

Methods: One hundred sixty-three lesions to the distal physis of the forearm bones in 157 patients were available for a long-term follow-up. Seventy-seven injuries of the distal radial physis were radiographically isolated, 54 were associated with a fracture of the ulnar styloid, and 26 with a fracture of the distal ulnar metaphysis. Of the six injuries of the distal ulnar physis, five were associated with a fracture of the distal radial metaphysis, and one was an isolated injury of the distal ulnar physis. All patients had both clinical and radiographic evaluation, with an average follow-up of 25.5 years (14-46 years). The average age of the patients at injury was 11.6 years $(5-17$ years), their average age at follow-up was 35.5 years (22-56 years).

Results: According to Salter and Harris, of the 157 radial lesions, 18 were type 1 and 139 type 2 . According to Ogden, 14 were type 1A, 4 type $1 \mathrm{C}, 84$ type $2 \mathrm{~A}, 13$ type $2 \mathrm{~B}, 17$ type $2 \mathrm{C}$, and 25 type $2 \mathrm{D}$. Of the 6 ulnar lesions, 2 were Salter and Harris type 1 (Ogden type 1A), 3 type 2 (Ogden type 2A), and 1 type 4 (Ogden type 4A). Fifty-four radiographically evident fractures of the ulnar styloid associated with injuries of the distal radial physis were classified as Ogden type 7A. At follow-up, all of our patients were fully asymptomatic, except for those who had forearm bone growth failure of more than $1 \mathrm{~cm}$. Shortening of the previously injured forearm bones ranging from 1 to $6.5 \mathrm{~cm}$ was observed in 2 open and subsequently infected lesions as well as in 5 uncomplicated lesions of the 157 distal radial physeal injuries, and in 3 of the 6 distal ulnar physeal injuries. Shortening of $1 \mathrm{~cm}$ or more was observed in the uncomplicated lesions of radial physeal injury with Ogden type $1 \mathrm{C}, 2 \mathrm{~B}$, and $2 \mathrm{D}$ lesions, and in ulnar physeal injuries Ogden type 1A, 2A, and 4A. Thirty-eight additional patients had radioulnar length discrepancy that ranged from 2 to $9 \mathrm{~mm}$, and 53 patients had styloid nonunion, but all of them were asymptomatic.

Conclusions: None of the patients at follow-up, including those with radioulnar length discrepancy of less than $1 \mathrm{~cm}$ and those with styloid nonunion, complained of any symptom related to their previous injury. Of the 10 patients with either radial or ulnar shortening of more than $1 \mathrm{~cm}$, only 2 with radial growth arrest and marked radioulnar length discrepancy had severe functional problems.

Significance: Growth disturbances of more than $1 \mathrm{~cm}$ following distal radial physeal injury occurred only in Ogden type 1 and 2 lesions, whereas in distal ulnar physeal injuries, growth disturbances occurred regardless of the Ogden classification type.

\section{2}

\section{TRAUMATIC NERVE LACERATIONS OF THE UPPER EXTREMITY IN CHILDREN} C. Plakogiannis, C. Papadopoulos, D. Petratos, E. Manoukas,
G. Matsinos, I. Anastasopoulos

Purpose: Nerve injuries are uncommon in children, hence not many studies exist in the literature dealing with this issue. We conducted a retrospective study aiming to evaluate the results of primary nerve repair in children and the factors affecting the clinical outcome.

Methods: From 1986 to 2004, 35 children from 1 to 13 years of age were treated in our department with lacerated ulnar and median nerves in the forearm and the wrist. 14 children were either visitors in our city or referred to our department from another part of the country and postoperatively were followed up in their local hospital. 19 children were followed up for 6 to 26 months and their medical records were available for review. We contacted 9 of these children, which were adults at the time of our study, and had a telephone interview with them. All children were operated within $24 \mathrm{~h}$ from the injury by 4 pediatric orthopedic surgeons and epineural neurorhaphy was performed. Limbs were immobilized in a long arm cast for 4 to 6 weeks (depending on the concomitant injuries). No formal rehabilitation regime was followed.

Results: There were 11 boys and 8 girls with a mean age of 9,2 years. The ulnar nerve was lacerated in 8 cases and the median nerve in 11. 9 injuries involved the left limb and 10 the right. All but one injury were caused by broken glass, in the house. In 6 cases there was no associated tendon or vascular injury (excluding isolated injury to palmaris longus). Using the MRC grading system recovery was excellent (M5, S4) in 7 children, very good in 8 ( $3 \mathrm{M} 5, \mathrm{~S} 3$ and $5 \mathrm{M} 4, \mathrm{~S} 3$ ), and good $(\mathrm{M} 3, \mathrm{~S} 3)$ in 4 cases. The most important factor affecting the outcome was the extent of the injury and the structures that needed to be repaired. Factors such as the age and the proximity of injury were not found to influence the outcome. The 9 adults that had a nerve injury during childhood and were interviewed over the telephone did not report any significant impact to their life from it. After an average of 12,9 years after their injury none had any additional reconstructive surgery. All coped well with the activities of daily living, six were employed as manual workers and three were office employees. Three complained of cold intolerance and one for an ugly scar. There was no essential change in their symptoms from what was documented in their notes at their last visit in the hospital.

Conclusions: Results of nerve repair in children are strikingly good. It seems that their increased regenerating potential together with the "plasticity" of their nervous system and its ability to adapt with changing conditions that occur following a peripheral nerve injury makes outcome so different compared to adults. Reeducation of a child is much easier and effective. 
Significance: Nerve injury of the upper limb may have devastating effects to the life of a child. Nevertheless early anatomic repair usually has very satisfactory results in this age group.

\section{3}

\section{TREATMENT OF OPEN FEMUR FRACTURES IN CHILDREN: COMPARISON BETWEEN EXTERNAL FIXATOR AND INTRAMEDULLARY NAILING}

\section{E. Ramseier, A. Bhaskar, A. Howard, W. G. Cole}

Purpose: Open femur fractures in children are uncommon and usually associated with other injuries. In adults there is a current trend to treat open fractures with intramedullary devices. The goal of this study was to compare external fixator (EF) to intramedullary devices (IM) in the treatment of open femur fractures in children.

Methods: Diaphyseal femur fractures without growth plate involvement were included. A total number of 35 patients (12 IM; $23 \mathrm{EF})$ were identified. Age, hospital stay, polytrauma, mechanism of injury, and Gustilo-Anderson Grade were recorded. Follow up was at least until the fracture was clinically and radiographically healed.

Results: Patients with external fixators were 5.2 times more likely $(95 \%$ CI $1.05,25.5)$ to have any complication. Excluding pin track infections, patients with external fixators were 2.7 times as likely $(.567,13.2)$ to have a complication Refractures occurred only in the external fixator group ( 6 of $23,26 \%$ ) and not in the IM nailing group $(P=0.062$, Fischer Exact Test). These were associated with varus malunions - all three of the external fixator group with more than $15^{\circ}$ of varus at fracture union suffered a refracture.

Conclusions: Treatment of open femur fractures in children is a challenging problem. Treatment with IM devices had fewer complications than the external fixator. We think when ever possible, especially grade I open injuries the use of IM devices for the treatment of open femur fracture in children should be considered. If external fixators are used, avoiding varus malunion my decrease the refracture rate and secondary change to an IM device should be considered.

\section{4}

\section{DISTAL TIBIAL PHYSEAL INJURIES; HOW COMMON IS PREMATURE PHYSEAL CLOSURE?}

\section{Thacker, A. Littleton, D. Thawrani}

Purpose: The incidence of premature physeal closure (PPC) in distal tibial physeal injuries is a topic of controversy. PPC has been reported in as low as $2 \%$ to as high as $38 \%$ cases. The aim of this study was to assess the incidence of PPC in distal tibial physeal injuries in our institution.

Methods: This was an IRB approved, retrospective cohort study of radiographs and clinical records of patients with distal tibial physeal fractures from January 2000 to June 2006. Of 632 ankle injuries treated during that time, 250 involved the distal tibial physis. Of these, 119 had average 16 months (6-82 months) follow up and were included in our study.

Results: The average age at injury was 12.2 years. The overall distribution of Salter Harris (SH) fractures was: SHI: 12, II: 29, III: 45, IV: 33. Sixty-one fractures were treated non-operatively and 58 required surgical intervention. The requirement of surgical intervention (I:0, II:5, III:30, IV:23) and incidence of PPC (I:0, II:2, III:3, IV:5) was higher in SH Type III and IV compared to Type I and II $(P<0.05)$. Ten of 119 patients $(8.4 \%)$ developed growth abnormalities (6 LLD and 4 angular deformities) despite near anatomic reduction. Of these 2 needed osteotomies for varus correction and one a contralateral epiphysiodesis for LLD $(1.8 \mathrm{~cm})$.

Conclusions: The incidence of PPC after physeal injuries requiring a secondary surgical intervention $(2.5 \%)$ is low. As expected, the incidence of PPC was highest in SH IV injuries (Triplane fracture). The high incidence of surgical intervention may explain these findings.

Significance: Premature physeal closure following distal tibial physeal injuries is low $(8.4 \%)$ with secondary surgical intervention needed in about $1 / 3$ of these.

\section{5}

\section{THE VALUE OF THE SONOGRAPHIC EVALUATION IN TRAUMATIC PATELLAR DISLOCATION IN ADOLESCENTS}

\section{J. M. Felus, B. Kowalczyk, T. Lejman}

Purpose: Traumatic patellar dislocation (TPD) may be followed by serious injuries determining treatment strategy. Superficial localization of the knee extensor apparatus structures and articular cartilage make them accessible to sonographic evaluation (USG). The purpose of this study is to analyze the value of knee sonographic evaluation in patients after TPD, prospectively.

Methods: 26 knees were examined sonographically after 1-14 days from the acute TPD. Sonographic evaluation followed clinical and radiographic examination. In all knees dynamic ultrasound examination of the patellar cartilage, as well as the medial patellofemoral ligament (MPFL) supplemented standard knee examination protocol. Based on USG findings the operative or conservative treatment was applied (17 and 9 cases, respectively). The criteria for surgery included: (1) statement of the presence of the floating loose body inside the joint cavity, (2) evidence of the complete rupture of the MPFL along with its functional insufficiency, or (3) significant MPFL edema in static examination and its distension in dynamic sonographic evaluation. In 2 cases treated conservatively the knee CT scans were taken to confirm sonographic diagnosis.

Results: In 19 knees the avulsive fractures of the patellar insertion of the MPFL were stated at sonographic evaluation. In 18 knees the lesions of the medial patello femoral ligament (MPFL) fibres were sonographically evident. Injuries of the femoral attachment of the MPFL or intramuscular hematoma of the VMO were present in 8 joints. 13 free osteochondral bodies and 1 nondisplaced osteochondral fracture were detected in 13 knees. Corresponding osteochondral lesions were localized in all cases. The presence of osteochondral loose body identified in USG was confirmed intraoperatively or in CT scan in $92,8 \%$, the osteochondral lesion localization in $85,7 \%$, avulsive fracture of the femoral attachment of the MPFL in $100 \%$. Generally the USG findings were confirmed by other methods in $84,2 \%$.

Conclusions: Ultrasound knee examination is an efficient method in the precise diagnosis of the extent of the injuries following traumatic patellar dislocation in adolescents. Additional data, crucial for decision making process are contributed by dynamic real time assessment of the articular surface of the patella and 
MPFL efficiency. The injuries following TPD are often multiple, therefore detailed evaluation of all the structures of the extensor apparatus as well as articular cartilage is mandatory.

\section{6}

\section{FETOSCOPIC RELEASE OF AMPUTATION THREATENING EXTREMITY AMNIOTIC BANDS}

F. Soldado, C. Amat, C. Fontecha, E. Carreras, J. Peiro, M. Aguirre, S. Arevalo, V. Martinez-Ibañez

Purpose: To report a case of a successful intrauterine limb salvage after extremity amniotic band (EAB) release of a leg at risk of amputation.

Methods: A 21 week gestational age fetus with amniotic band syndrome diagnosed by ultrasound and MRI was treated by fetal surgery. A minimally invasive surgery technique was used. Uterus was approached by single port fetoscopy. Amniotic band was released by direct fetoscopic vision and ultrasound guide with a longitudinal extremity incision, sectioning the constriction band, using a Nd:Yag-Laser.

Results: Prenatal Ultrasounds and Echo-doppler follow-up showed a progressive increase of limb arterial flow and decrease of distal edema. The fetus was born at 28 weeks of gestational age after a spontaneous preterm delivery showing a viable limb with a severe leg constriction. Definitive surgery was performed at 4 weeks after birth with residual band excision and multiple Z-plasties.

Conclusions: EAB release is a feasible temporary procedure to avoid an intrauterine amputation. Definitive band excision would be performed after delivery. Premature rupture of membranes and prematurity would be the main complication of this treatment.

Significance: Selected cases of antenatally diagnosed EAB are amenable of fetal surgery.

\section{7}

\section{USE OF RHBMP-2 IN CONGENITAL PSEUDARTHROSIS OF THE TIBIA}

\section{B. S. Richards, C. E. Johnston, M. W. Shrader, R. D. Welch}

Purpose: To determine the outcomes of patients with congenital pseudarthrosis of the tibia treated with rhBMP-2.

Methods: Seven patients (age range 1.9-11.7 years) underwent Williams intramedullary rod fixation and autogenous bone grafting. Collagen sponges, saturated with rhBMP-2, were placed circumferentially around both the nonunion site and bone graft. The dose of BMP-2 ranged from 4.2 to $12 \mathrm{mg}$. Per body weight, the doses ranged from 0.16 to $1.11 \mathrm{mg} / \mathrm{kg}$. Postoperative cast immobilization averaged 15 weeks (range 9-29 weeks). Four of the 7 patients had neurofibromatosis. Two of the 7 patients had undergone previous surgery in earlier attempts to repair the pseudarthrosis.

Results: Follow-up averaged 2.2 years (range 6 months5.9 years). Four rods crossed the ankle joint and three were fixed in the distal segment only (augmented with interlocked $2 \mathrm{~mm}$ screws). Postoperatively, there were no adverse effects from the BMP-soaked collagen sponge. Radiographic and clinical union was achieved in five of the seven patients (BMP dose range $4.2 \mathrm{mg}-12 \mathrm{mg}$ ). Time to radiographic union in which the fracture site was remodeled averaged 29 weeks (range 16-53 weeks). One of these five patients subsequently re-fractured through a site distal to the original pseudarthrosis. Revision rodding, grafting, and BMP-2 augmentation in that patient again resulted in healing 18 weeks later. Of the remaining 2 patients (BMP dose 8.4 and $12 \mathrm{mg}$ ), one is 10 months postoperative and without solid radiographic union. The other had persistent nonunion after further surgery and eventually underwent amputation.

Conclusions: Success was achieved in five of seven patients who underwent Williams intramedullary rod fixation, autograft, and augmentation with rhBMP-2 on a collagen sponge. The BMP and collagen sponge are well tolerated at the site of the congenital tibial pseudarthrosis. The $6-8.4 \mathrm{mg}$ dose seems to be the appropriate range for most patients using the collagen sponge carrier. With the additional use of rhBMP-2, the time needed to achieve a radiographically confirmed union is noticeably reduced when compared to the current literature using Williams intramedullary rodding and bone graft alone.

Significance: The use of rhBMP-2 on a collagen sponge to augment Williams intramedullary rodding and bone grafting for congenital pseudarthrosis of the tibia appears to be beneficial.

\section{8}

\section{DEVELOPMENT OF HUMAN HIP JOINT CONTAINMENT IN SECOND AND THIRD TRIMESTER OF PREGNANCY; CADAVERIC STUDY}

\author{
A. Masoń, A. Borowski, A. Grzegorzewski, J. R. Bowen, \\ M. Synder, M. Topol
}

Purpose: The potential etiology of developmental dislocation of the hip still remains as a dilemma. Previous studies described development of the hip joint in their first and second fetal period; however, there are no reports in literature of dislocated hips in fetuses earlier than third trimester. To better understand etiology of developmental dysplasia of the hip we tried to study pre-natal development of human hip joint during their second and third trimester of pregnancy. The aim of this study is to determine hip joint morphology in fetus, and containment changes during pregnancy.

Methods: To study the development of hip joint during the fetal period, histologic serial sections were made through 23 cadaver infants (46 hips). Measurements of neck shaft angle (NSA), femoral antetorsion angle (FAA), acetabulum anteversion angle (AAA) and acetabulum slope angle (ASA) have been performed. All measurements above were performed on cadaver species photography taken on femur and pelvis. To standardize pelvis position, $\mathrm{K}$ wire was inserted through obturator foramen, next to the anterior obturator tubercle, on the obturator crest of the superior ramus of the pubis. Femur was positioned on the table in a regular manner to detect femoral version with both femur condyles placed on a flat surface. The femoral shaft length (FSL) and proximal and distal epiphysis width were cared out on the basis on $\mathrm{X}$ ray in order to calculate appropriate fetus age. Finally, to asses pre-natal development of human hip joint, FSL/NSA ratio, FSL/FAA ratio, FSL/ASA ratio and FSL/AAA ratio were calculated.

Results: The mean lunar age was 7.6 month, ranged from 5 to 10 months. The hip developmental ratios were plotted for all cadaveric species. The NSA did not show any significant changes with time. The average NSA was 119.8. SD 26.2. The slope of FSL vs. NSA was $-0.628, \mathrm{R}^{2}=0.0091$. Correlation index was -0.095 . It was a plateaus slope pattern. The FAA ranged from 15 to $33^{\circ}$. The slope of FSL vs. FAA was $0.197, \mathrm{R}^{2}=0.13$. Correlation 
index was 0.36 . It was an upward slope pattern. The AAA ranged from 6 to $35^{\circ}$. The slope of FSL vs. AAA was $0.3966, \mathrm{R}^{2}=151$. Correlation index was -0.133 . It was a downward slope pattern. The ASA ranged from 51 to $85^{\circ}$. The slope of FSL vs. ASA was $0.397, \mathrm{R}^{2}=0.152$. Correlation index was 0.388 . It was a downward slope pattern.

Conclusions: The NSA does not change significantly during second and third pregnancy trimester. The FAA increases during pregnancy, correlates with age. The AAA as well as ASA shows trends to decrease during second and third pregnancy trimester, but do not correlate with age.

Significance: Only the FAA's changes correlates with age during second and third trimester of pregnancy. The AAA and ASA do not play important role in hip joint containment during second and third trimester of pregnancy.

\section{9}

\section{ACCOMPANYING PATHOLOGIES, KNEE STRUCTURES AND TREATMENT METHODS IN CONGENITAL DISLOCATION OF THE KNEE}

\section{N. A. Okan, M. M. Orak}

Purpose: Our aim in this study is to discuss the accompanying pathologies, knee structure and applied treatment methods in our cases of congenital dislocation of the knee (CDK).

Methods: 9 patients with $\mathrm{CDK}$ were consulted to us within 1 year. 7 of them were newborns. There were 5 girls and 4 boys. 12 knees were affected.6 patients' 9 knees were type 1;3 patients' 3 knees were type 2 .We have not observed type $3 \mathrm{CDK}$ in our series. 3 patients were with arthrogryposis multiplex congenita having teratologic hip dislocations; one had both sided clubfeet either. Two had spina bifida with one sided and both sided clubfeet respectively. One hemihypertrophy, one congenital short femur with fifth finger aplasia at the same side; one clubfoot and one calcaneovalgus deformity at the reverse side were seen. Mothers of two CDK had uterus didelphus. We performed manual streching for type 1 and serial casting for the arthrogrypotics and type 2 cases followed by orthosis application ranging 3-6 months long.MRI were taken for type 2 knees. One patient with arthrogryposis underwent quadricepsplasty. Minimum follow-up time was 1 year.

Results: Type 1 patients were treated by manual stretching within 2 weeks- 1 month and the result was excellent. After 2-6 cast applications, full range of motion was achieved in type 2 cases and $70-90^{\circ}$ knee flexions were maintained in arthrogrypotics. For the prevention of hyperextension tendency in type 2 and for continuation of the achieved knee flexion in arthrogrypotics, we applied knee orthosis 3-6 months after casting. Type 2 knees were stabilized well. Arthrogrypotics were refractory. We performed quadricepsplasty to one of our arthrogrypotic patients. Result was successful with the maintenance of $90^{\circ}$ flexion. MRIs have shown normal structure in type 2 knees.

Conclusions: Foot anomalies were not the most common pathologies accompanying CDK in our series. Neither intrauterine malpositions, nor DDH were seen except the ones with arthrogryposis. Maternal uterus abnormalities were seem to be the cause of CDK. Type 1 cases were easy to treat by manual stretching and type 2 by serial casting at newborn period. Because of the hyperextension tendency after maintaining full range of motion in type 2,we recommend orthosis application with extension stop at least 3 months for stabilization.CDK with arthrogryposis were refractory to the conservative treatment. Early surgical treatment should be planned for them. Knee structures of type 2 cases have shown no abnormalities in MRI in contrast with the literature. Significance: We emphasize that CDK could be treated easily by conservative methods at newborn period except the ones with arthrogryposis. Without intrauterine malpositions, DDH may not accompany CDK so frequently. Maternal uterus didelphus should be examined carefully as a cause of CDK. Pathologies in knee structures might be the result of some delay in treatment.

\section{0}

\section{THE TREATMENT OF THE SEVERE MULTI-PLANAR DEFORMITY OF THE PROXIMAL PART OF THE FEMUR IN CHILDREN}

\author{
A. A. Sakalouski, O. V. Kovalchuk, Y. V. Likcacheuski
}

Purpose: The treatment of the severe multi-planar deformity of the proximal part of the femur is a very difficult orthopedic problem Methods: We used intertrochanteric osteotomy with posterior 45$90^{\circ}$ or anterior $45-70^{\circ}$ rotation of the proximal part of the femur proposed by A.M. Sakalouski in 114 cases (112 patients): posterior -103 and anterior -11 . Surgical treatment was performed in cases of residual deformity of the femoral head for dysplasia-69 cases, Perthes disease-19, postseptic deformity-13, slipped capital femoral epiphysis - 6, posttraumatic avascular necrosis -4 and another disease - 3 . The age of our patients ranged from 2 years 10 months to 17 years. The indications for surgery were: severe damage or deformity of the superior segment of the femoral head and severe multi-planar deformity of the proximal part of the femur. The aims of the surgical intervention were elimination of load on the damaged or deformed superior segment of the femoral head, restoration of the femoral head centralization, restoration of articular surface congruity, normalization of the greater trochanter position, lengthening of the femoral neck and the limb.

Results: We used different methods of assessment for different diseases, but in most cases clinical results were graded according to McKay's system and radiological results-Severin's system, as modified by Zionts and MacEwen. Good and excellent results were registered in $87 \%$ of all the cases.

Conclusions: The posterior or anterior rotation osteotomy has a following merits: reorientation of the femoral head in three dimensions, absence of negative influence on the growth plate of the femoral head, and moreover, positive influence on the femoral head growth plate of the due to restoration of normal biomechanical conditions, absence of angle deformities, lengthening of the femoral neck and the limb.

Significance: Thus, unlike traditional osteotomies, rotational osteotomy permits to change the femoral head position in three dimensions and is very helpful in many severe cases which may seem hopeless at first sight.

\section{1}

\section{INCREASED FREQUENCY OF PAI-1 GENE POLYMORPHISM IN LEGG-CALVE-PERTHES DISEASE: PRELIMINARY RESULTS OF A PILOT STUDY}

D. Yazgan Aksoy, A. Gurgey, I. C. Haznedaroglu, M. Alpaslan, M. C. Aksoy

Purpose: The etiopathogenesis of Legg-Calve-Pethes disease is stil not clear. A self-limited process which results in the avascular 
necrosis of the femoral head causes chronic disabilities. Many hypothesis including inflammation, infection, trauma, hypercoagulation had been postulated in the past. Plasminogen activator inhibitor 1 (PAI-1), a single-chain glycoprotein is the primary inactivator of tissue plasminogen inhibitor which converts the inactive protein plasminogen into plasmin; the critical molecule in fibrinolysis, cell migration and epithelial cell differentiation. PAI-1 limits the production of fibrin and increased risk of bleeding. A common functional deletion/insertion polymorphism $(4 \mathrm{G} / 5 \mathrm{G})$ in the promotor of of the PAI-1 gene is associated with elevated expression of PAI-1 gene. The prevelance of $4 \mathrm{G}$ allele was found to be higher in disorders like coronory heart disease, severe preeclampsia, pulmonary thromboembolism, osteonecrosis and some forms of arterial thrombosis. We previously demonstrated increased tissue factor pathway inhibitor and thrombomodulin levels along with increased global fibrinolytic capacity in this unique disease which could only be explained as a defense mechanism against ongoing hypercoagulation. The aim of this study was to determine if there is a genetic defect in PAI-1 gene in Legg-Calve-Perthes Disease.

Methods: 17 patients with LCPD and sex and age-matched healthy controls were included in the study.

Results: 13 of them is found to be $4 \mathrm{G} / 5 \mathrm{G}$ heterozygote $(76 \%), 3$ $4 \mathrm{G} / 4 \mathrm{G}(18 \%)$ homozygote and $15 \mathrm{G} / 5 \mathrm{G}(6 \%)$ homozygote. The percentages were $40 \%$ for $4 \mathrm{G} / 5 \mathrm{G}, 26 \%$ for $4 \mathrm{G} / 4 \mathrm{G}$ and $34 \%$ for $5 \mathrm{G} / 5 \mathrm{G}$. $4 \mathrm{G} / 5 \mathrm{G}$ prevalence was significantly high in LCPD $(P<0.001)$.

Conclusions: The association between PAI-1 gene polymorphism and thrombosis is still controversial. The high prevalence of the $4 \mathrm{G} / 5 \mathrm{G}$ variant may be an explanation for underlying possible thrombosis in LCPD which might indirectly result in increased parameters of fibrinolysis.

Significance: This study may enlighten the reason of thrombosis in LCPD.

\section{2}

\section{PRESENTATION AND OUTCOME OF CONSERVATIVELY TREATED PATIENTS WITH LEGG-CALVÉ-PERTHES DISEASE}

\section{Froberg, F. Christensen, N. Wisbech Pedersen, S. Overgaard}

Purpose: We aimed to compare the modified lateral pillar classification with the results of the Stulberg outcome defined by Herring.

Methods: From 1941 to 1962, 167 patients with LCP disease were treated at The Community of Disabled in Kolding, Denmark. 143 patients had unilateral involvement and 24 patients had bilateral involvement. Retrospectively the medical records of the patients were retrieved and gender and age at debut was registered. All hips were treated conservatively by a Thomas splint. Patients with bilateral involvement $(n=24)$ or insufficient or missing radiographs $(n=69)$ were excluded. For 74 patients (61 boys and 13 girls) with unilateral involvement the original radiographs still exist. The average age at onset of the disease was 6.2 years. Radiographs from the fragmentation phase of the disease were classified according to the modified Herring lateral pillar classification and head-at-risk (HAR) signs were noted. At skeletal maturity radiographs were classified according to the Stulberg classification system.

Results: In group A and B there were 35 Stulberg I or II results and 12 III, IV or V. In group $\mathrm{B} / \mathrm{C}$ and $\mathrm{C}$ there were 15 Stulberg I and II, and 12 Stulberg III, IV and V. The association between Herring class and Stulberg outcome was significant $(P=0.041)$. The Herring class and Stulberg group were analyzed with respect to age at debut. We did not find a significant association of age at onset and Herring group $(P=0.411)$, nor could we find a significant association of age at onset and Stulberg group $(P=0.060)$ though there seemed to be a tendency of children older than 9 years at debut to end in Stulberg III, IV or V. $71 \%$ of patients in group A have HAR signs, $80 \%$ in group B, $100 \%$ and $93 \%$ in group $\mathrm{B} / \mathrm{C}$ and $\mathrm{C}$ respectively. The mean number of HAR signs was increasing the worse Herring group $(P=0.02)$. There were not a significant association between number of HAR signs and Stulberg group $(P=0.594)$. $64 \%$ of the boys was classified to Herring A and B and $70 \%$ in Stulberg I and II. In the female patients $62 \%$ were Herring $\mathrm{A}$ and $\mathrm{B}$ and $62 \%$ ended in Stulberg I and II. There was no significant association between gender and Herring class $(P=0.346)$ or Stulberg group $(P=0.094)$.

Conclusions: The Herring class correlated to the Stulberg group. A significant association between patient age at onset and Stulberg outcome was not found. The small sample sizes in this study raise the possibility that significant results were missed. We regard presents of HAR signs to be a potential risk to end in a poor Herring group more than an indicator for a poor Stulberg outcome. Gender did not influence on the prognosis.

Significance: Linear regression analysis was used to determine which combination of factors affected the probability of a certain Stulberg outcome. A significance level of $p<0.05$ was chosen for all calculations.

\section{3}

\section{LATE ONSET PERTHES DISEASE}

\section{T. Wirth}

Purpose: Patients who develop Legg-Calvé-Perthes disease (LCPD) at a relatively old age are known to have bad results at the end of the growth period due to limited remodeling potential. We evaluated the outcome of treatment in patients suffering from LCDP with a late onset beyond the age of 9 years at the end of growth.

Methods: From 1999 to 200422 patients were identified to have late onset LCDP beyond the age of 9 years. They were followed throughout their disease and finally evaluated at skeletal maturity with use of the Stulberg classification and the functional outcome by use of the Harris hip score. According to Joseph et al. the radiographs of all patients were grouped as follows: classical course of the disease, segmental collapse, and head destruction without ability of regeneration. The treatment modalities were recorded.

Results: Among the 22 patients 20 were male and 2 female. The age at onset of the disease was 9 years in 1,10 years in 3, 11 in 5, 12 in 7, 13 in 4 and 15 and 16 in one patient each. We found 9 patients with a classical course, 8 with segmental collapse and five hips with head destruction. There were 25 surgical procedures performed: Containment treatment in 9, valgisation osteotomies in 5 , trimming of the femoral head in 4 , drilling in 1 and distraction by external fixation in 2 patients. One patient had a Chiari osteotomy, 3 patients needed total hip replacement and 4 were treated conservatively. The overall clinical outcome was bad with an average Harris hip score of 45 points. The Stulberg classification revealed one patient in Group II, three patients in 
Group III, seven patients in Group IV and eleven patients in Group V.

Conclusions: Late onset LCPD is one of the most difficult diseases to treat in adolescence. The result of any hip preserving treatment is disappointing in most patients. Only those patients who follow the natural course of the disease have a chance to get acceptable results.

Significance: All treatment options for patients with late onset LCPD lead to unsatisfactory outcomes in most patients. More detailed classifications are needed to adapt the best treatment for the patients. We may actually deal with a separate orthopedic entity.

\section{4}

\section{SURGICAL TREATMENT OF LEGG-CALVÈ-PERTHES DISEASE; IMPROVED RESULTS FOR SEVERE FORMS CATTERALL IV/HERRING C}

\section{A. Diméglio, F. Canavase, M. Ali, P. Kelly}

Purpose: The purpose of our study was to analyse our results of surgical management of severe forms of Legg-Calvè-Perthes disease.Catterall has reported that the natural history for untreated severe forms of Legg-Calvè-Perthes disease, Catterall III/IV, is associated with a poor result in $91 \%$ of cases. For Herring surgical treatment of does not influence the outcome of Herring $\mathrm{C}$ type hips. Conway has shown that isotope bone scans can detect severe forms of Perthes disease earlier than with conventional radiographs.

Methods: A selective series of 69 hips between 6 and 10 years of age classified as Herring $\mathrm{C} / \mathrm{Catterall} \mathrm{IV} \mathrm{with} \mathrm{total} \mathrm{head} \mathrm{necrosis}$ were analyzed and reviewed at skeletal maturity. There were two treatment groups; conservative group; treated with range of movement exercises and non-weight-bearing until the reconstructive phase and the surgical group; treated with a pelvic osteotomy. There were 27 hips in the conservative group and 42 hips in the surgical group. All hips were classified as Catterall IV/ Herring $\mathrm{C}$ and Conway $\mathrm{B}$ or $\mathrm{C}$ on isotope bone scan. Both groups were comparable in relation to age and sex. All hips were reviewed at skeletal maturity and classified according to the Stulberg classification.

Results: There was a significant improvement in outcome between the surgically treated group and the conservatively treated group $(P<0.005)$. In the conservative group there were 12 $(44.5 \%)$ Stulberg I/II, 5 (18.5\%) Stulberg III and $10(37 \%)$ Stulberg IV/V. In the surgical group there were $25(59.5 \%)$ Stulberg I/II, 8 (19\%) Stulberg III and 9 (21.4\%) Stulberg IV/V. Further analysis of our 42 cases treated surgically revealed 17 hips that had surgery less than 7 months following diagnosis. This early surgical group had 14 Stulberg I/II, 3 Stulberg III with no cases of Stulberg IV/V.

Conclusions: In severe forms of Perthes disease, we have shown that surgery can alter the natural history and produce better results than conservative management. Isotope bone scan can detect cases earlier than conventional radiology.

Significance: Treatment of Herring C/Catterall IV hips with a pelvic osteotomy can lead to superior radiological and clinical results at skeleatal maturity if performed early, prior to femoral head deformation. Serial isotope bone scans allows early detection of severe forms.

\section{5}

\section{LEGG-CALVÉ-PERTHES DISEASE: THE ACETABULULAR DEVELOPMENT FOLLOWING PELVIC OSTEOTOMY}

\section{A. J. Schreiner}

Purpose: Pelvic osteotomies with the aim of containment increase are a central pillar of the operative LCPD management. The effect of pelvic osteotomies on the outcome in the statistical overview has been studied several times. Occasionally surprised by the unexpected development of the acetabulum following a pelvic osteotomy I studied systematically not only the femoral but also the respective acetabular evolution in individual cases.

Methods: Longstanding X-ray evolutions of patients in two major pediatric orthopedic centers in Austria and Switzerland were studied. There were 45 hips fulfilling the criteria of a completed disease process. The observation protocol included the sphericity of the femoral head as a matter of course, but also the inclination of the acetabular roof, the reasons for salvage operations and the effect of operations carried out in the course of the active phase of the disease.

Results: In 22 of those 45 hips an operation was done for sequel of the disease. 12 of those operations consisted in a pelvic osteotomy in order to correct an increased inclination of the acetabular roof in cases of Stulberg III. In 3 of the 12 patients being operated on for increased inclination of the acetabular roof another pelvic osteotomy for the purpose of containment increase was done before during the active phase of the disease. The effect of the operation on the acetabulum had resolved completely in the further course of the disease.

Conclusions: The purpose of pelvic osteotomies as a part of the containment increasing strategy is the prevention of a steep acetabular roof as a consequence of the acetabulum's adaptation to the expanding femoral head. In the observed cases the increase of this inclination could not be prevented by a pelvic osteotomy. This study shows that there are cases when nature does not only decline to follow the intention of the operators with respect to the effect of the increased containment on the femoral head, but also with respect to the effect on the acetabulum itself.

Significance: Studies on management outcome have made it clear that there is only a particular group of patients to benefit of containment increasing operations in form of pelvic osteotomies. This study shows that we cannot even be sure whether our pelvic osteotomy meets the aim of containment increase.

\section{6}

\section{TRIPLE PELVIC OSTEOTOMY IN LEGG-CALVE-PERTHES DISEASE USING A SINGLE INCISION: A FOUR-YEAR REVIEW}

\section{E. Conroy, D. McCormack, J. Harty, M. Timlin}

Purpose: Femoral head incongruency at skeletal maturity is associated with the development of osteoarthritis in early adulthood. Containment of the femoral head provides a larger surface area for remodelling of the collapsed femoral head and the development of spherical congruency. Triple pelvic osteotomy has a role to play in Legg-Calve-Perthes disease by improving femoral 
head containment and preventing subluxation. This is traditionally a two incision approach with significant associated morbidity. In our unit we perform triple osteotomies through a single anterolateral incision. We retrospectively reviewed clinical and radiographic outcome of children who had triple osteotomies carried out through a single incision over a 4 -year period.

Methods: In our unit from 2003 to 2006 we performed eight triple osteotomies through a single incision in children aged between 6 and 12 years with Legg-Calve-Perthes disease. The procedure was performed through a single anterolateral incision made beneath the middle of the iliac crest and carried forward as per a Salters osteotomy. Image intensification was used to confirm iliac, pubic and ischial cuts. After performing a standard Salter's osteotomy the acetabular fragment was free to rotate anteriorly and laterally. None of the children were casted and all were allowed immediate mobilization non weight bearing with crutches for 6 weeks.

Results: Clinical results and hip function were measured pre and post operatively using the modified Harris hip score. Average length of hospital stay was 4.7 days. None of the children had a non union. The centre edge angle of Wiberg was measured on all pre and post operative AP pelvic radiographs. In all our patients there was an improvement in the centre edge angle of Wiberg and in the modified Harris hip score. The pre operative modified Harris hip scores ranged from 38 to 60 and postoperatively ranged from 77 to 92 . The pre operative centre edge angle of Wiberg ranged from 9 to 24 while post operative scores ranged from 25 to 46 .

Conclusions: Triple osteotomy has been advocated in Legg-CalvePerthes disease following closure of the triradiate cartilage. Using a single incision is a safe alternative to the traditional two-incision approach.

Significance: We believe that the single incision approach reduces operative time and potential morbidity associated with the Steel triple osteotomy with comparable clinical and radiographic outcomes.

\section{7}

\section{CHIARI OSTEOTOMY AND SHELF ACETABULOPLASTY IN THE TREATMENT OF SEVERE PERTHES' DISEASE}

\author{
M. Batouty, W. Adl, Y. Kandeel
}

Purpose: This paper compares the clinical and radiographic results of Chiari osteotomy and shelf acetabuloplasty for treatment of severe Perthes' disease.

Methods: Twenty-five children suffering from unilateral severe Perthes' disease with hip pain, limping, and deformed uncontainable femoral heads were included in this study. Treatment in 13 children was by Chiari osteotomy, and in 12 children by shelf acetabuloplasty. The mean age at surgery was 8.9 years for Chiari osteotomy, and 9.16 years for shelf acetabuloplasty. The clinical and radiographic data of the two groups were reported at the time of surgery and at follow-up after surgery.

Results: There were no significant differences of the preoperative clinical and radiographic data between the two groups. The mean follow-up was 4.38 years for Chiari osteotomy, and 4.08 years for shelf acetabuloplasty. Of the 13 Chiari osteotomies, 10 had good results by Robinson et al. clinical grading, 1 had fair result , and 2 had poor results. There were highly significant improvements in Sharp's angle and the percentage of acetabular cover $(P<0.001)$. The changes in the femoral head size ratio, the acetabular depth index, the height index, and the total depth index were insignificant $(P>0.05)$. For the 12 shelf operations, there were 8 good and 4 fair results. There were highly significant improvements in Sharp's angle and the percentage of acetabular cover $(P<0.001)$. The changes in the femoral head size ratio, the acetabular depth index, and the height index were insignificant $(P>0.05)$. The shelf length and the total acetabular depth index decreased significantly $(P<0.001)$ at the final follow-up.

Conclusions: Both Chiari osteotomy and shelf acetabuloplasty improved hip pain and mobility and provided adequate femoral head coverage. Both techniques did not interfere with the growth of the acetabulum. Although the acetabular shelf reduced over time, but the final femoral head coverage provided by both techniques was not significantly different.

Significance: Both Chiari osteotomy and shelf acetabuloplasty are effective salvage procedures for treatment of severe Perthes' disease with deformed uncontainable femoral head.

\section{8}

\section{EXPERIMENTALLY-INDUCED SCOLIOSIS BY COSTOTRANSVERSECTOMY IN MINIPIGS. TWO MODELS OF SPINAL TENSEGRITY BREAKAGE COMPARING MIDLINE VERSUS POSTEROLATERAL SURGICAL APPROACH}

\author{
O. Riquelme-García, A. González-Miranda, C. Barrios, \\ C. Correa, E. Hevia, J. Burgos, J. González-López
}

Purpose: Costotransversectomy has probed to be a reliable method for induction of scoliotic deformities in animals with research purposes. An alteration of the spinal tensegrity has been recently proposed as the pathogenic mechanism for curve progression. Two different models of spinal tensegrity breakage through either midline or posterolateral surgical approaches were investigated.

Methods: Seven minipigs (mean age 6-week) underwent costotransversectomy at 5 consecutive vertebral segments. In 4 animals ribs removal (T7-T11) was performed through a posterior midline approach and in other 3 by a posterolateral approach (T6-T10) without desinsertion of paraspinal muscles. Thoracic spine deformity was assessed by standard X-rays at the immediate postoperative period, and at 2 and 4-month follow-up. Anatomic specimens were also macroscopically studied just at sacrifice 5 moths after surgery.

Results: All 4 animals operated on by midline posterior approach developed structural spinal deformity with curve convexity at the side of rib removal. Mean Cobb angle was found to be $34,6^{\circ}$ (2741). Vertebral rotation was clearly evident in these 4 animals. Animals operated on by posterolateral approach without paraspinal muscle desinsertion did not develop any significant spinal deformity.

Conclusions: As in smaller experimental animals, costotransversectomy was a reliable method for induction of scoliosis in minipigs. Scoliotic deformity showed a similar appearance than human idiopathic curves. Damage of the posterior muscle-ligamentous structures around costotransverse joints breaking of the spine tensegrity is mandatory to induce scoliotic deformity. Rib removal alone appeared to have less scoliotic inductive implication.

Significance: To our knowledge no previous studies have focused breakage of the spinal tensegrity by an experimental model in which costotransverse joints are removed with minimal damage of the paraspinal muscles. The conclusion that muscle-ligamentous structures are more decisive than rib resection for curve progression questions previous knowledge on scoliosis etiopathogenesis. 


\section{9}

\section{GROWING ROD INSTRUMENTATION AND VERTEBRAL BODY GROWTH. A RADIOLOGICAL INVESTIGATION IN IMMATURE PIGS}

\section{G. Yilmaz, C. Ozkan, G. Demirkiran, G. Huri, K. Daglioglu, M. Yazici}

Purpose: Distraction forces applied on growth plate of appendicular skeleton stimulate longitudinal growth. However the effect of distraction forces on axial skeletal growth has not been fully investigated yet. The aim of this study is to evaluate the vertebral body growth under distraction forces in immature pigs treated with growing rod technique.

Methods: Eight 8 week-old domestic pigs were used in this experimental model to simulate growing rod instrumentation technique. Cranially T12-L1 and caudally L4-L5 vertebrae were instrumented by pedicle screws bilaterally, while L2 and L3 were skipped. Distraction between pedicle screws was applied at index surgery. The rods were then lengthened twice in a month interval. All subjects were evaluated with lateral spinal X-ray preoperatively, postoperatively and at the final follow-up. The vertebral body heights of distracted segments (HD $=$ L2 and L3) and control segments $(\mathrm{HC}=\mathrm{T} 9, \mathrm{~T} 10$ and $\mathrm{T} 11)$ were measured. Average vertebral body heights and the percent increase in the vertebral body heights were compared among control segments $(n=11)$ and distracted segments $(n=8)$.

Results: Four subjects were lost during the immediate postoperative period. The preoperative vertebral body height was similar in two groups (HC: $10.97 \mathrm{~mm}$, HD: $11.27 \mathrm{~mm}, P>0.05$ ). At the final follow-up; the average vertebral body height in distraction group was significantly higher than the control group (HC: $16.92 \mathrm{~mm}$ HD: $18.56 \mathrm{~mm}, \mathrm{p}<0.05)$. The percent increase in vertebral body height was higher in distracted segments but there was no statistically significant difference between the two groups Conclusions: The vertebral growth continues during growing rod instrumentation. Distraction forces might stimulate also apophyseal growth of axial skeleton.

Significance: The distracted segments during growing instrumentation in early onset scoliosis continue their longitudinal growth. Their longitudinal growth might be even more than non-distracted segments due to distracted forces applied to growth plates of vertebrae.

\section{0}

\section{FETAL SURGERY OF MYELOMENINGOCELE BY FETOSCOPY IN THE OVINE FETUS}

\section{Fontecha, F. Soldado, J. Peiro, J. Sevilla, M. Aguirre, V. Martinez-Ibañez}

Purpose: To asses the utility of prenatal fetoscopic coverage of the defect and show that fetoscopic treatment can reproduce the same results than the open surgery, but with fewer rates of complications.

Methods: We created a surgical myelomeningocel defect in the spine of 27 sheep fetuses, at the middle of the gestation, resecting the posterior elements of the 3 lumbar vertebras and opening the dura mater. Eighteen animals (group A) underwent only this surgical procedure. Nine animals (group B) underwent fetoscopic coverage of the defect using inert material 3 weeks later.

Results: Survival rate was $50 \%$ in group A and $56 \%$ in group B. Animals from group A showed a defect of closure in the lumbar area, leakage of spinal fluid, paraplejia of lower limbs, incontinence of sphincters and Chiari II malformation. Animals from group B showed a small scar in the lumbar area, normal neurological status of limbs and sphincters and normal cranio-cervical junction.

Conclusions: Foetoscopic coverage of myelomeningocel improves the neurological situation at birth in lambs. This technique is a good alternative to prenatal open reparation.

Significance: Minimally invasive surgery can expand the indication for fetal surgery in MMC.

\section{1}

\section{NATURAL HISTORY OF EARLY THORACO-LUMBAR KYPHOSIS IN CHILDREN WITH ACHONDROPLASIA}

\section{W. G. Mackenzie, A. Littleton, B. Borkhuu, G. Chan}

Purpose: Thoracolumbar kyphosis (TLK) is a frequent skeletal deformity in patients with achondroplasia. Limited studies have been published on the natural history of thoracolumbar kyphosis and sagittal alignment in patients with achondroplasia.

Methods: We retrospectively reviewed the medical records and radiographs of 45 children with achondroplasia. Patients were included if they were referred for initial evaluation before age 2 with specific concerns regarding kyphosis. All lateral radiographs were analyzed for the thoraco-lumbar kyphosis, thoracic kyphosis (T5-T12), lumbar lordosis (below TLK-S1), both the height and width of apical vertebral wedging, and apical vertebral translation. Medical records were reviewed for gender, the age of the onset of walking, presence of ventricular-peritoneal shunt, presence of developmental motor delay, and brace treatment (if any). Two groups were created based on the presence or absence of spontaneous resolution (TLK $<20^{\circ}$ ) of the deformity.

Results: There were 31 patients ( 15 male and 16 female) who resolved spontaneously by 3 years of age and 17 patients ( 5 male and 12 female) who did not. Fourteen patients were braced due to concerns of kyphosis. Patients who resolved began walking at an average age of 17.9 months compared to 22.7 months for those who did not $(P=0.017)$. Developmental delay was present in $56 \%$ of patients who did not resolve as compared to $23 \%$ who did $(P=0.049)$. Wedging of the apical vertebral height was $78 \%$ in the group that did not resolve, and $60 \%$ in the group who did resolve $(P=0.016)$. Apical vertebral translation was present in significantly more patients who did not spontaneously resolve $(41 \%)$ compared to those who did $(6 \%)$. Lumbar lordosis below the kyphosis was $67^{\circ}$ in the group that did resolve and $75^{\circ}$ in the group that did not $(P=0.036)$.

Conclusions: Spontaneous correction of thoraco-lumbar kyphosis is seen in patients with achondroplasia. Persistent thoraco-lumbar kyphosis is most common in patients who begin to walk late, have developmental delays, have apical vertebral wedging, and apical vertebral translation.

Significance: Spontaneous correction of thoraco-lumbar kyphosis is seen in patients with achondroplasia. Persistent thoraco-lumbar kyphosis is most common in patients who begin to walk late, have developmental delays, have apical vertebral wedging, and apical vertebral translation. 
52

\section{SCOLIOSIS ASSOCIATED WITH CONGENITAL DIAPHRAGMATIC AGENESIA REPORT OF A LARGE SERIES (41 CASES)}

\section{A. Antaranyan, B. Dohin , C. Garin, D. Gorduza, R. Dubois, R. Kohler}

Purpose: Agenesia or aplasia of the diaphragm is one of the possible congenital defects of the diaphragm. Improvement of anesthesiology and intensive care of newborns led to a drop in death rate in the early childhood, which in its turn brought about the necessity to supervise the natural history of the disease. One problem is the development of deformation of the spinal column which is rarely reported.

Methods: Out of 89 patients with diagnosis of diaphragmatic hernia, 41 patients with the agenesia/aplasia of the diaphragm were treated and supervised in our department between 1987 and 2007. 21 of the 41 patients were males, 20 were females. 34 cases were diagnosed as agenesis of the diaphragm upon pre-natal ultrasound examination, and 7 cases upon the post-natal radiological examination. The infants were born at the age of $27-41$ gestation weeks. The surgery was performed on $0-8$ post-natal days, generally on the 1st-2nd day. More often, the defect was closed with a muscular flap, and in case of doubts about surgical suture tension, the suture was armored by VICRIL ${ }^{\circledR}$ mesh. 18 of them died. The 23 surviving children were followed with particular interest on spinal deformities. The follow-up was performed by pediatric surgeons, including radiological exams at 6 month and 1 year of age, and hereafter every other year. In case of spinal problems shown by radiological examination, the patient was examined by orthopedic surgeons.

Results: 10 patients were under periodic supervision by an orthopedist, 6 of whom were treated with the help of Milwaukee and TLSO types of brace, as well as plaster casts. The mean follow up was 10.1 years (1 to18 years). The mean angle of curvature before treatment was $23^{\circ}\left(8-45^{\circ}\right)$ and $25^{\circ}\left(8-58^{\circ}\right)$ at the end of treatment. The supervision of 4 patients who did not receive conservative treatment was limited to a radiological examination. 2 patients with respectively $55^{\circ}$ and $60^{\circ}$ of spinal curvature were operated on. A posterior arthrodesis of spine by using the CotrelDubousset expandable system was accomplished.

Conclusions: The rate of development of scoliosis is quite important and requires careful observation. We advocate a multidisciplinary follow up (pediatric surgeons, orthopedic surgeons). When scoliosis appears conservative treatment should be performed which may prevent surgical correction.

Significance: Children suffering of agenesia or aplasia of the diaphragm must be carefully observed by pediatric surgeons and orthopaedic surgeons in order to diagnose deformation of the spinal column.

\section{3}

\section{SURGICAL CORRECTION OF SPINAL DEFORMITIES AFTER HEART TRANSPLANTATION: A CASE SERIES REPORT}

\author{
D. P. Roye, B. Kaufman, J. A. Gomez, J. E. Hyman, \\ L. Addonizio, M. G. Vitale
}

Purpose: Several studies have demonstrated the relationship between congenital heart disease and scoliosis. More recent research has shown that children who undergo solid organ transplantation are also at an increased risk for developing scoliosis. It is important to review the cases of patients undergoing scoliosis correction after orthotopic heart transplantation and establish any necessary changes in their perioperative care to ensure good surgical results. Methods: Between 1995 and 2007, six heart transplant recipients with diagnosis of scoliosis required corrective spinal surgery. Two patients had congenital heart disease, 2 had congenital cardiomyopathy and one had Marfan's syndrome. Through a retrospective chart review preoperative cardiac status as well as preoperative Cobb angles where obtained from the surgeon's notes. Intraoperative and follow-up information was also reviewed. All of the patients included in the study received orthotopic heart transplantation, four patients underwent a posterior spinal fusion and instrumentation, one patient underwent a posterior segmental instrumentation without fusion and the last patient had an anterior release and posterior fusion with instrumentation.

Results: The average age at time of heart transplantation was $7.8 \pm 4.5$ years. These children underwent surgical correction for their scoliosis at a mean age of $13.3 \pm 5.6$ years. Average length of follow-up after the scoliosis surgery was $21.5 \pm 14.7$ months. Two of the patients in the cohort are deceased, as a result of their cardiac condition. Three patients had complications; one of them fractured a single rod construct another patient had a device migration after initial surgery and one patient required treatment for a wound infection. None of the complications resulted in adverse sequelae. Appropriate curve correction and control was achieved. The mean preoperative major curve was $70.2^{\circ}$, and at follow up the largest mean curve was $22.6^{\circ}(P<0.10)$.

Conclusions: This multiple case report demonstrates that spine deformity surgery can be successfully performed in children and adolescents who have undergone a heart transplant. This is accomplished with meticulous preoperative planning, intraoperative and post operative team care all performed in an environment where multidisciplinary cooperation and care of this difficult group is routine.

Significance: As demonstrated by this group of patients, spine deformity surgery can be successfully performed in children and adolescents who have undergone a heart transplant; this is accomplished with meticulous preoperative planning, intraoperative and post operative team care all performed in an environment where the care of this difficult group is routine.

\section{4}

\section{VERTEBRAL MANIFESTATIONS IN CRMO}

\section{Langendoerfer, F. Fernandez, T. Hospach, T. Wirth}

Purpose: Chronic non-bacterial osteomyelitis is an aseptic form of osteomyelitis with several synonyma such as CRMO and SAPHOSyndrom describing an inflammatory disorder of unspecified origin. Manifestations of CRMO in the spine appear to be quite frequent, ranging from 'silent' affection to severe alterations of the vertebral body, ending in a 'vertebra plana'.

Methods: Between 2002 and 200725 children with spinal manifestations due to CRMO were seen. Some were primarily treated because of their osteomyelitic vertebral manifestations, some showed minor vertebral affections in addition to their main osteomyelitic foci. All patients were diagnosed with conventional radiographs in two planes and with MRI of the spine or full bodyMRI. The diagnosis of the CRMO was always established by a biopsy, usually at relevant, easy-to-reach sites. 
Results: In 25 patients treated due to CRMO, spinal manifestations of varying degree were found. Three different types of vertebral manifestations were found: Type I with a mild manifestation of osteomyelitis, mostly clinically 'silent'. Type I is characterized by significant signal alterations in MRI, yet showing no anomalies in conventional radiographs. This pattern was found in 9 out of 25 patients. Type II shows a moderate manifestation of osteomyelitis, which was diagnosed by significant signal alterations and signs of destruction of the vertebral body in the MRI, as well as by anomalies in the conventional radiographs. In 10 out of 25 cases irregularities in the end plates or compression deformity of the vertebral body under $10^{\circ}$ were found. One of these cases showed atypical, isolated sclerosis of a vertebral body like in Garré's disease with MRI signal alterations of several other vertebral bodies at the same time. Most of the patients showed moderate degrees of back pain and were sufficiently treated with naproxen. Type III shows a severe manifestation of osteomyelitis, which was found in 6 out of 25 patients. The conventional radiographs and the MRI showed compression of the vertebral bodies with more than $10^{\circ}$ wedging. Two cases developed a total collapse of the vertebral body, e.g., a 'vertebra plana'. Treatment was a combination of bisphosphonates, TNF-alpha-antagonists and temporary bracing.

Conclusions: Vertebral manifestations of CRMO show a great variation in their clinical appearance. In many cases they may happen even unnoticed or may be described as a 'silent' focus in MRI. Whereas most of these 'silent' Type I manifestations vanished in later MRIs, some of these may progress into Type II or III manifestations. These should be monitored more closely, to prevent severe vertebral body destruction by early and adequate pharmacotherapy.

Significance: There is a strong correlation between conventional radiographs, MRI and clinical appearance of the vertebral manifestations of CRMO suggesting three types or groups in the given collective.

\section{5}

\section{PROSPECTIVE STUDY INTO SHORT-TERM INTRAVENOUS ANTIBIOTIC TREATMENT OF ACUTE SEPTIC ARTHRITIS AND ACUTE OSTEOMYELITIS IN CHILDREN}

\section{R. Kanwar, C.E. Bache, H.K. Graham}

Purpose: Septic arthrits and osteomylelitis have traditionally been managed by intravenous antibiotics for 4-6 weeks. This requires a prolonged in patient stay, inconvenience to parents, morbidity and unnecessary cost. A number of authors have suggested that shortened course of intravenous antibiotics 7-10 days are effective. Our aim was to evaluate a shortened 3 day course of intravenous antibiotic regime.

Methods: In 2001 we started to prospectively evaluate a shortened 3 day regime of intravenous antibiotics. We prospectively treated 36 cases of acute osteomyelitis and 30 cases of acute septic arthritis in children. These were confirmed by positive blood culture, positive aspirate culture, raised WCC in joint aspirate for septic arthritis or positive bone scan/culture for osteomyelitis. These patients were treated with a shortened course (3 days) of intravenous antibiotics following surgical drainage when required. Serial measurements of inflammatory markers and clinical status were recorded. On day 4 of admission if clinical and biochemical parameters improved patients commenced high dose oral antibiotics. If no improvement they continued IV abx and consideration for repeat washout given. Patients were discharged on a 3 week course of antibiotics. They were followed up at 1 week, 3 weeks, 6 weeks, 3,6 and at 12 months following discharge. Inflammatory markers were checked in OPD until normal. Serial X-rays to survey growth disturbance and arrest. Discharge at 1 year most infection if no ongoing problems. Outcome measures that were analyzed: duration of IV administration, inpatient stay, readmission/reoccurrence.

Results: 43 of the $66(66 \%)$ patients received were discharged by day 5 after receiving 3 full days of intravenous antibiotics. Mean inpt stay was 5.5 days. There was one re-admission for intolerance of high dose antibiotics. 6 septic patients required a repeat washout (day 4-7 of admission). At 3 months there were no patients with ongoing infection. (Based on inflammatory markers). At 1 year no child had evidence growth arrest of radiological examination.

Conclusions: We suggest the vast majority of acute suppurative skeletal infection can be managed safely with shortened course of intravenous and oral antibiotics following surgical drainage (in the case of intra articular infection). About $25 \%$ of patients will need longer courses of antibiotics and possibly repeat washout. Significance: Shorter impatient stay is preferable due to cost reasons, decreased morbidity of Intravenous access, inconvenience to patient and parents. Our study highlights a subgroup of patients that can be identified by careful clinical evaluation and measurement of inflammatory markers and managed with a shortened 3 day regime of intravenous antibiotics.

\section{6}

\section{COXAPLASTY IN HIP RECONSTRUCTION FOR TYPE II LATE SEQUELAE OF SEPTIC EPIPHYSITIS: A PRELIMINARY REPORT}

\section{H.M. El Tayeby}

Purpose: To suggest different procedures tailored for hip reconstruction in type II late sequelae of septic epiphysitis. The severely deformed subluxated or dislocated head is reshaped in accordance to radiographic and on table assessment.

Methods: Material and methods: 16 hips in 13 patients ( 3 being bilateral) were the subject of this study. They were affected during the first few weeks from birth (all were incubated). Age at operation was 2-12 years. The period of follow up ranged from 2 to 5 years. Plain radiographs, CT, and multi-slice CT with reconstruction views were beneficial in analyzing the problem preoperatively. Approach: A modified Smith Peterson approach was used and will be described to adequately expose the iliac bone (for acetabular reconstruction) and the hip and upper half of femur (for femoral shortening, capsular fashioning, global femoral head reshaping). Debridement and capsular fashioning: Meticulous dissection to well preserve the amalgamated capsule should be planned for later adequate capsulography. Debridement of the acetabulum from fibrous tissue is attempted. Head and neck reconstruction (Coxaplasty): the deformed-hypertrophied (coxa magna) femoral head was addressed by a carefully planned reshaping in a manner not disturbing the usually unhealthy superior weight bearing surface (Red zone). This will allow easy containment in the reconstructed acetabulum. Associated subluxation or dislocation will dictate adequate shortening (up to $5 \mathrm{~cm}$ ) as an essential step for a successful containment. Acetabular reconstruction: Acetabular dysplasia is usually circumferential needing Dega acetabuloplasty or less commonly anterolateral needing Salter osteotomy. Tripple acetabular osteotomy for better caping was performed in older children. 
Results: According to the criteria proposed by Hunka a satisfactory result is considered when a stable pain free hip is achieved with flexion arc more than 70 and flexion contracture less than $20^{\circ}$. This was true in 13 hips. A final improvement in the range of motion should not be expected before 6-12 months. Intensive physiotherapy to overcome post operative stiffness is required.

Conclusions: A carefully planned coxaplasty + adequate femoral shortening + appropriate acetabular reconstruction in hips with type II sequelae of septic epiphysitis will secure an unexpected stable, painless, more or less fully mobile hip. To achieve such a result certain criteria should be respected during dissection and reconstruction.

Significance: Coxaplasty for hip reconstruction should be attempted as a salvage procedure. This will achieve a stable painless more or less mobile hip , and will facilitate hip replacement if ever needed in the future.

\section{7}

\section{MUSCULOSKELETAL LANGERHANS CELL HISTIOCYTOSIS IN CHILDREN: ANALYSIS OF PATIENTS OVER THREE DECADES}

\section{J. P. Dormans, A. Arkader, H. Hosalkar, L. Moroz, M. Glotzbecker}

Purpose: To analyze the clinical presentation, features, treatment, and prognosis of primary musculoskeletal disease in children with Langerhans cell histiocytosis.

Methods: A database of $\mathrm{LCH}$ cases diagnosed at a tertiary referral center between 1972 and 2003 was retrospectively reviewed to identify children with primary bone involvement. Cases were categorized as spinal involvement or extraspinal involvement. Spinal lesions with collapse were further classified as Grade I ( $<50 \%$ collapse) or Grade II (50-100\% collapse), and subclassified as A (symmetric) or B (asymmetric). Extra-spinal involvement was categorized by the number of bones involved $(1,2$, or 3 and more), and whether or not there was systemic involvement. All children had a bone scan or skeletal survey to search for additional sites.

Results: Among $160 \mathrm{LCH}$ cases, 70 children had primary musculoskeletal disease. There were 26 children with primary spine involvement and 46 with extraspinal involvement. The mean age of the spine group was 8.2 years. Fifteen children had single vertebral involvement while 11 had multiple. There were 20 IA lesions, 3 IB, 10 IIA, and 9 IIB. The most common presenting symptom was pain (17 patients) followed by neurologic symptoms (3 patients). Ten children were treated with chemotherapy, four with radiation therapy, and two with both. Four children developed a spinal deformity, two of whom required fusion. The mean age of the 46 patients in the extraspinal group was 7.6 years. A single bone was involved in 32 cases, 2 bones in 4 cases, and 3 or more bones in 10 cases. Eleven of 46 patients $(24 \%)$ had systemic involvement, and of these, three patients were diagnosed with diabetes insipidus. Presenting symptoms in this group included pain (32), a palpable mass (5), high fever (3), torticollis (2), otitis media (2), and pathologic fracture (2). Seventeen cases were treated with curettage, 8 with chemotherapy, one with radiation therapy, and 15 with a combination of chemotherapy and radiation therapy.

Conclusions: There is variability of presentation in musculoskeletal lesions of LCH. Biopsy is almost always indicated to confirm the diagnosis. Although the natural history for most lesions is that of gradual healing, simultaneous curettage and grafting is often indicated to accelerate the healing process. Internal fixation for stability is occasionally necessary in the appendicular skeleton. Chemotherapy is usually used for multisystem disease, and radiotherapy is contraindicated and no longer used.

Significance: To describe the presentation, treatment evolution, and outcomes of patients with primary musculoskeletal Langerhans cell histiocytosis over three decades.

\section{8}

\section{EOSINOPHILIC GRANULOMA OF THE BONE: A DIAGNOSTIC ISSUE}

\author{
A. Andreacchio, A. Brach del Prever, A. Linari, A. M. Postini, \\ C. Defilippi, M. Berta, M. Chiavola, M. Pagano
}

Purpose: Langerhans cells' Histiocytosis is a proliferative disorder of reticuloendothelial system of inflammatory nature. The disease involves bone, skin, lymph nodes or parenchymal organs as liver, spleen, lungs, bone marrow and causes endocrinological disorders or neurological deficit.There is a clinical spectrum ranging from solitary bone lesion to severe multisystem involvement. Monosystemic disease (typically involving bone) can regress spontaneously (especially monosthotic) or can need specific treatment but normally it has good prognosis. On the other hand multisystemic forms have a worst prognosis and it is necessary systemic treatment with chemotherapy and steroid drugs.

Methods: Bone is often involved: $60 \%$ of patients affected present at least one bone localization, especially for flat and short bones of the trunk, followed by long bones of upper and lower limbs.It is difficult to differentiate eosinophilic granuloma (solitary skeletal lesion) from other bone diseases or systemic diseases with secondary bone involvement. At diagnosis common is pain, associated to dysfunction or not. Conventional X-ray shows osteolysis area which destroys osseous trabeculae and erodes the cortex; typical imagine is "a stampo" similar to a cockade but sometimes it can resemble malignant lesions: for instance in diaphysial localizations, periosteal reaction can produce an "onion's bulb" imagine which is to differentiate from Ewing's sarcoma or osteomyelitis. When the vertebral body is involved and X-ray shows typical "vertebra plana" aspect, it is fundamental follow the lesion evolution in order to have a correct diagnosis. On the onset the lesion aspect is lytic without collapse of vertebral body, then partial or complete compression of the body occurs until the stabilization of the lesion.It is necessary do not forget that similar aspects occur in Gaucher's disease, lymphomas, undifferentiated sarcomas and in osteogenesis imperfecta as well. CT scan and MRI have lower specificity compared to radiography; bone scintigraphy has lower sensibility. Biopsy permits the definitive diagnosis: Langerhans histiocytes are characterized by the presence of CD1A and S100 Antigens and at electronic microscopy Birbeck's granules are visible as well.

Results: Between June 1968 and May 2007 in our Centre 118 patients have been followed: 77 males $(65 \%)$ and 41 females (35\%).In $74 \%$ of cases the disease involved a single system: $63 \%$ of these had monofocal bone lesions, $20 \%$ multifocal ones; on the other hand, $61 \%$ of multisystemic patients had almost one bone localization. $4 \%$ of monofocal and $35 \%$ of multifocal bone patients presented relapse of disease. $16 \%$ of patients, all monofocal, have been followed with "wait and see"; $19 \%$ (monofocal or multifocal) underwent curettage, the others chemotherapy. Conclusions: All the patients with monofocal and multifocal bone disease are alive at 10 years from diagnosis. 
Significance: Bone disease has good prognosis by specific treatment.

\section{9}

\section{DIAGNOSIS OF OSTEOID OSTEOMAS WITH A 3D TIME-RESOLVED MRI ANGIOGRAPHY}

P. Journeau, A. Lecocq, L. Mainard-Simard, M. Claudon, P. Lascombes, T. Haumont

Purpose: 3D time-resolved MRI angiography is a non invasive method of imaging which enables the diagnosis of osteoid osteomas with $100 \%$ certitude related to the specificity of the arterial curve. Our series concerns 10 consecutive osteoid osteomas evaluated with this method.

Methods: A prospective study of 10 children suspected of osteoid osteomas of the long bones was performed. The children had a 3D dynamic angio-MRI. All the patients had both clinical symptoms and radiological signs which led to suspect osteoid osteomas. The study of the arterial vascularisation of the tumour permitted us to determine its special pattern. In order to validate the method, the patients had a CT scan and a bone scan te99 examinations.

Results: In every case, the pre operative diagnosis of osteoid osteoma was suspected because of the characteristic aspect of the MRI. It was always confirmed by pathological study. The specificity of the angio MRI is the early arterial vascularisation of the nidus, below some milliseconds. Then, the peripheral inflammatory reaction to the tumour appeared. Thus, it was possible to describe a specific pattern of the arterial vascularisation of the osteoid osteomas.

Conclusions: The arterial curve of the nidus is specific in angio MRI and is reproducible in all the prospective studied cases. This method is reliable and precise. Another advantage is the capacity to have a mapping of an osteoid osteoma, to precise its relations with the peripheral anatomical structures.

Significance: The specificity of the arterial curve pattern in angio MRI of osteoid osteomas leads to the diagnosis of osteoid osteomas. This allows the clinician to avoid other examinations such as CT scan and/or bone scan.

\section{0}

\section{PERCUTANEOUS RESECTION OF OSTEOID OSTEOMA (73 CASES) A 20 YEAR EXPERIENCE}

\section{R. Kohler, B. Dohin, I. Canterino}

Purpose: Osteoid osteoma is a benign tumor requiring excision because of pain, usually severe. Treatment by percutaneous drill resection with $\mathrm{CT}$ scan guidance has been developed for 20 years as an alternative method to the classical "en bloc" resection (DOYLE, VOTO, KOHLER). This is a report of a series of 73 cases, the largest one reported so far.

Methods: The procedure is performed in the CT scan room by the orthopaedic surgeon, assisted by the radiologist for CT guidance. The lesion (nidus) is thus localized on an appropriate slice, after correct positioning of the patient. A special set of cannulated instruments (drill, trephine, reamer) are introduced in succession along a Kirschner wire through a short skin incision. A small cylinder of bone is removed for pathological analysis. 73 patients were treated from June 1987 through June 2007. There were 58 children and adolescents (2.5-18 year) and 15 adults (19-35 year). All patients had clinical and radiological evidence of osteoid osteoma (hyperfixation on scintigraphy and visible nidus on CT scan according to Campanacci criteria). The nidus was mostly located in the lower limb (66 cases) especially the femoral neck (26), femoral shaft (14), tibial shaft (21).

Results: No severe complications were observed: slight skin burn of the leg in 4 cases, transitory palsy of extensor hallucis longus in one case. No fracture was to be deplored. The nidus in the removed specimen was isolated in $60 \%$ of the cases. Clinical healing (after a 1 year follow up) was observed in all but 3 cases: 1 "true" recurrence and 2 technical failures - for which a second attempt was successful.

Conclusions: Percutaneous resection has now clearly demonstrated its safety and effectiveness. Benefits for patients are evident: quick recovery and immediate full weight bearing, consequence of minimal bone resection. The method is now indicated in most cases, while "en bloc" resection should be considered only for some vertebral or superficial localizations of the nidus. Alternatively, other techniques quite similar to resection (destruction by thermo coagulation or photocoagulation) have been reported: they are all non invasive interventional procedures but seem more costly and a higher rate for recurrence is reported.

Significance: Quality and consistency of results on such a large series encourage recommending percutaneous resection for treating osteoid osteoma.

61

\section{EPIPHYSIAL SPARING TECHNIQUES FOR MALIGNANT TUMORS IN CHILDREN USING EPIPHYSIAL DISTRACTION (PAMPLONA TECHNIQUE) AND TRANSEPIPHYSIAL RESECTIONS}

\section{G.U. Exner, S. Jürgen}

Purpose: Joint sparing is a prerequisite for biologic reconstructions allowing for permanent healing in bone tumors. The physis not crossed by vessels in children can provide a safe margin for tumor resection. In selected patients we have performed joint sparing procedures either by transepiphysial resection or by epiphysial distraction as introduced by Canadell and San Julian.

Methods: 8 children (1 Ewing tumor, 8 osteosarcomas [1 multiple localizations]) with open physes ( 3 distal femurs, 6 proximal tibiae) were treated for metaphysial tumor localizations touching but not crossing to the physis were treated. In 4 localizations epiphysial distraction was used, in the others transepiphysial resection. Reconstructions were performed with vascularized fibula alone in 4 cases, with vascularized fibula transfer and allograft in 1 patient, in the others only intercalary allografts were used. Results: $\mathrm{F} / \mathrm{u}$ is $2-11$ years. No local recurrence occurred. One intercalary allograft failed for infection after irradiation; this was salvaged by a modified rotation plasty. One patient with fibula reconstruction of the femur needed reosteosynthesis due to lack of fusion with a finally excellent result at 3 years $f / u$, in one the fractured allograft needed be replaced by autologous bone, but the epiphysis could be retained. All functions well except for the growth deficiency due to the loss of the physis. One patient already had lengthening for the correction of leg length discrepancy. Conclusions: Epiphysial sparing tumor resection can be successful oncologically if patients are properly selected and surgery is respecting the tumor margins. 
Significance: This technique allows joint sparing, when other procedures (e.g., endoprosthetic replacement) need to sacrifice a joint thus allowing for true biologic reconstructions.

\section{2}

\section{DIAPHYSEAL RECONSTRUCTION AFTER TUMORAL RESECTION OF LONG BONES IN CHILDREN USING INDUCED MEMBRANES TECHNIQUE. PRELIMINARY REPORT OF 5 CASES}

\author{
B. de Billy, B. de Courtivron, C. Bonnard, J. Langlais, \\ M. L'Kaissi, M. Planchenault
}

Purpose: The aim of the study is to present the first cases of diaphyseal reconstruction in two steps with the technique describes by Masquelet, after tumoral resection. Though this technique was initially described for traumatic loss of substance, we applied this procedure to tumoral pathology.

Methods: Five children (one girl and four boys) with a mean age of 7,9 years $(3-11,5)$ underwent diaphyseal reconstruction. Diagnoses were Osteosarcoma in one case and Ewing Tumor in four cases. Localizations were humerus in one case, femoral diaphysis in three cases and neck and upper extremity of the femur in another case.

Results: After tumoral resection a spacer was inserted (one metallic spacer and four cement spacers hold by elastic pins). Total weight bearing was allowed after 6 weeks. The second step was performed after 9 months $(5-15)$ for the lower limbs localizations and after 21 months in the case of humeral tumor. Spacers were removed and bone graft from tibia and iliac crests were placed. Stabilization was obtained by pins ( 2 cases) centromedullary locked nail ( 2 cases) and LCP plate ( 1 case). Total weight bearing was allowed after 2 months except for the femoral neck reconstruction. Consolidation allowing material removal was obtained within 2 years. Complications were: pins replacement in 2 cases, pseudarthrosis of the proximal part of the graft in 2 femoral proximal reconstructions. They have been treated by new graft. One of the femurs was fractured after material removal. Leg length discrepancy were seen in 2 cases.

Conclusions: These preliminary results show a good rate of consolidation. In this short series complication rate seems to be equivalent other techniques of diaphyseal reconstruction like vascularized fibula transfer. Quality of bone seems very interesting

Significance: We would like to emphasize the simplicity of this new procedure and the absence of morbidity of donor site.

\section{3}

\section{COMPLICATIONS OF EXTENDABLE PROSTHESIS IN CHILDREN WHO SURVIVE LONGER THAN 10 YEARS}

\section{F. Chotel, 1. Jeys, R. Grimer, R. Tillman, S. Abudu, S. Carter}

Purpose: Extendable endoprosthesis allow maintenance of equal limb length in skeletally immature patients with primary bone tumours. We report the results and complications of 82 patients who have survived their cancers at a minimum of 10 years following implantation of extendable prostheses.

Methods: Between 1979 and 1996 a total of 134 skeletally immature patients received extendable endoprosthesis at our centre.
This study is a review of 82 patients who have survived their disease for longer than 10 years after surgery. All the patients have reached skeletal maturity. The rest of the patients were excluded because 49 had died from disease and 3 were alive but had early amputation because of local recurrence. The mean age at time of surgery was 9.7 years (range $3-15$ ). The sex ratio male/ female was 50:32. The initial diagnosis was osteosarcoma for 50 patients (pts) and Ewing sarcoma for 23. All prosthesis were cemented and 22 of them had a HA collar. The location was distal femur (43), proximal femur (7), total femur (1), proximal tibia (22), proximal humerus (6), and total humerus (3). Three types of extension mechanisms have been used: Ball bearing (23), C-Ring (31) and Minimal invasive (28). The total number of lengthening procedures was 576 , with a mean of 5.3 procedures per prosthesis. Sixteen prosthesis were routinely revised because at end of the lengthening mechanism.

Results: The mean follow-up was 14 years (range 10-25 years). $82 \%$ of the patients had undergone one or more revision, $48 \%$ of patients had two or more revision, and $16 \%$ three or more. The average patient had 1.7 surgical procedures for treatment of complication. 139 surgical procedures were required for complications including aseptic loosening (40), septic problems with one or 2 stage revision (32) fracture of implant or adjacent bone (21), mechanical problem due to the prosthesis (19) and rebush (12), and fixed flexion deformity or extensor problems (14). The long term rate of infection was $29 \%$ (24 patients). Seven patients $(8.5 \%)$ were amputated because of uncontrolled infection (5) or continuous pain unexplained (2). The introduction of hydroxyapatite collar has led to significant reduction of aseptic loosening and/or fractures of the implants from 80 to $57 \%$. Infection rate in the minimally invasive prosthesis was similar to the older designs. Conclusions: The rate of complications after extendable prosthesis remains very high but the limb salvage is still achieved in more than $90 \%$ of the patients who have survived their disease.

Significance: Infection remains a concern even with the minimally invasive design. Perhaps the non-invasive design will reduce the risks and complications.

\section{4}

\section{MANAGEMENT OF LIMB DEFORMITIES FOLLOWING USE OF VASCULARISED FIBULA AFTER TUMOUR RESECTION IN SKELETALLY IMMATURES}

\section{H. Abdel-Ghani, H. El-Barbary, W. Ebeid}

Purpose: In this study we present our experience in management of non union, mal union or LLD or combination of these problems that may complicate free vascularised fibular graft (VFG) or pedicled fibular graft (PFG) done to reconstruct long bone defects of the lower limbs after tumor resection. The line of management of these problems is not well established. Little if any literature discussed these problems.

Methods: We operated on 11 patients, average age 13 year, 5 males and 6 females with history of osteosarcoma of distal femur (6 cases), proximal tibia (4 cases) and distal tibia ( 1 case). The cases were treated with perioperative chemotherapy, tumor resection (radical resection in 9 cases with fusion of the nearby joint and condylar sparring resection around the knee in 2 cases) and primary reconstruction with either VFG or PFG. All the patients had LLD and mal alignment of the lower limbs with either mal union or non union of the fibular graft. We used Ilizarov fixator for lengthening and to correct mal alignment with either closed reduction or compression of non union sites or 
corrective osteotomy at sites of mal union avoiding doing osteotomy through the fibula.

Results: Solid union was achieved in all non union cases. Leg length equalization was obtained in condylar sparing resection cases and indented LLD of $1-2 \mathrm{~cm}$ shortening in knee and ankle fusion cases. Complications included, pin tract infection, delayed bone consolidation and stress fracture of the fibula that occurred in 4 cases that healed well within 3 months.

Conclusions: Difficult fixation of vascularised fibula in immature patients after tumor resection makes it liable to some complications. Excessive scarring and disturbed anatomy in these patients mandated surgery with minimal exposures. We should avoid doing osteotomies or inserting wires or half pins through the fibula. Closed alignment and fixation of non union sites and compression with lengthening of nearby bone is satisfactory to achieve union. Acute correction of mal alignment was easily achieved in all cases. Intramedullary fixation with thin nails in conjunction with the Ilizarov helps to maintain alignment and facilitate the operative procedure. Prolonged protection in brace is mandatory to avoid stress fracture of the non hypertrophied fibula.

\section{5}

\section{RESULTS OF TREATMENT OF EWING'S SARCOMAS AT A SINGLE INSTITUTION IN SWITZERLAND}

\section{A. Krieg, F. Hefti, M. Paulussen}

Purpose: We made a retrospective analysis of 32 Patients that were treated for Ewing's Sarcoma (ES) in a multidisciplinary approach between 1988 and 2006 at our institution. The purpose of the study was to assess the survival rates and to identify prognostic factors of patients with ES in comparison with other reports.

Methods: All tumors were resected by the same surgeon. Surgery was combined with radiotherapy in $56.2 \%$ of the patients ( $n /$ Emphasis $>=18)$. Neo-adjuvant chemotherapy was administered in all patients according to the actual international protocol: CESS $86(n=12)$, EICESS $92(n=8)$, EURO-EWING 99 $(n=12)$. 5-year and overall survival rates were calculated by using the Kaplan-Meier method. Median age at diagnosis was 16.4 years $(3.5-59.5) .53 \%$ of the tumors were located at the trunk. Results: The average follow-up time for all patients was 6.3 years (7.3 years for survivors, 3.4 years for non-survivors). Good response to chemotherapy was found in $78.1 \%(n=25)$ compared with $21.9 \%$ non-responders $(n=7) .5$ year-survival was $79.0 \%$ and the overall survival rate was $75 \%$. There was a significant difference between patients with metastases $(n=10)$ compared those without $(n=22 ; P=0.02)$. Clinical relevant predictors are the chemotherapy protocol, the response to chemotherapy and the age.

Conclusions: The survival rate was superior to those reported in multicentric studies using the same protocols with a comparable patient collective. This is typical for a single institution report with an experienced surgeon. Presence or absence of macroscopic metastases was the major prognostic factor, response to chemotherapy and age was less predictive while (in contrast to other reports) large tumor size and location at the trunk were not negative factors.

Significance: There are only a few reports in the literature but single center/single surgeon seems to be a good prognostic factor. The survival rate is superior to those reported in multicentric studies.

\section{DISCRETE EVENT SIMULATION FOR POLICY DECISION MAKING AND OPTIMIZED IMPLEMENTATION IN SCREENING STRATEGIES FOR DEVELOPMENTAL DYSPLASIA OF THE HIP}

\section{S. Ramwadhdoebe, C. P. Uiterwaal, E. Buskens, R. J. Sakkers}

Purpose: Previously routine ultrasound (US) screening for developmental dysplasia of the hip (DDH) was proven to be cost effective in the Netherlands. However, actual implementation of new strategies in (health care) organizations is an additional hurdle. A new strategy may imply organizational changes and possibly has external effects. To analyze potential problems before a "trial and error" implementation scenario commences, preferably a system analysis should be performed. Quite efficiently obstacles and bottlenecks may be identified and alternatives explored. A tried method for performing system analyses is to develop a simulation model. We will use a simulation model to evaluate the cost-effectiveness of the implementation of a new US screening strategy for detecting $\mathrm{DDH}$, while simultaneously identifying an optimal organization of care.

Methods: Over 4500 children will be screened with US in youth health care organizations in different regions in the Netherlands. Thus data are collected on mutually interactive system characteristics such as location, travel time, number and type of professionals and number and type of appliances required while also taking into account a rural setting as opposed to an urban setting. A so called discrete event simulation model (DES) will be used to calculate the cost effectiveness in the various environments. An important feature of DES is the possibility to explicitly incorporate the interaction between events and to analyze the process in more detail, e.g., in terms of cues (waiting times), resource use and effects thereof on clinical outcome.

Results: With the use of DES and the data of the implementation study of general US screening for DDH in more than 4,500 children in various environments in the Netherlands we will not only be able to calculate the costs and the possible cost-effectiveness of this general US screening for DDH in other parts of the Netherlands but also be able to determine the capacity required in terms of personnel and locations. Moreover, also the funds required for national implementation may be estimated.

Conclusions: DES will provide relevant information that will ultimately enable health authorities and local decision makers to change the screening policy towards DDH on the basis of accurate qualitative and quantitative simulation data.

Significance: With DES, implementation strategies for detecting dysplasia of the hip (DDH) can be optimalized.

\section{7}

\section{BILATERALLY DISLOCATED HIPS TREATED WITH THE PAVLIK HARNESS ARE NOT AT HIGHER RISK FOR FAILURE}

\section{A. Borowski, A. Littleton, D. Thawrani, L. Grissom, M. Thacker}

Purpose: Bilateral hip dislocations have been perceived to be associated with a higher risk for failure of Pavlik harness treatment. The purpose of this study is to evaluate the efficacy of Pavlik harness treatment in patients with bilaterally dislocated hips, and to compare them to cases with unilaterally dislocated hips treated with the same protocol. 
Methods: Twenty-nine patients (58 hips) met the following inclusion criteria: presentation with bilateral dislocated hips with no prior treatment, and dynamic Ultrasound (US) examination. The comparison group consisted of thirty-eight patients (38 hips) with unilateral hip dislocation treated with the same protocol. US examination was used to determine the reduction and stability within 3-6 weeks post Pavlik harness application. Successful treatment was defined as relocation/reduction of the hips within 3 weeks of harness application. Lack of reduction needing open/ closed reduction was considered a treatment failure. Standard demographic data and complications were recorded.

Results: The average age at the time of Pavlik harness application was 16 days in the bilateral group and 24 days in the unilateral group. The average duration of Pavlik wear was 109 days in the bilateral group and 92 days in the unilateral group in patients successfully treated with the harness. $17 / 29$ patients $(59 \%)$ in the bilateral group ( 7 bilateral, 10 unilateral) and 22/38 patients $(58 \%)$ in the unilateral group failed harness treatment.

Conclusions: Patients presenting with bilaterally dislocated hips treated with the Pavlik harness are at no greater risk for failure than patients presenting with unilateral hip dislocation. There was no difference in the duration of harness wear for successful patients regardless of presentation.

Significance: Patients with bilateral hip dislocations were not found to have a higher risk of failure of harness treatment in this study.

\section{8}

\section{THE ROLE OF ACETABULAR CARTILAGE THICKNESS ON ACETABULAR DEVELOPMENT IN DEVELOPMENTAL DYSPLASIA OF THE HIP}

O. Kalenderer, A. Bicimoglu, H. Agus, H. Omeroglu, Y. Tumer

Purpose: In this study, effect of the morphological structure of the acetabular cartilage on the acetabular development were tried to be determined by ages group and measuring the thickness of acetabular cartilage on the artrographies performed during the reduction of dysplastic hips through medial approach.

Methods: This prospective and multicentre study includes 106 hips of 78 children with developmental hip dysplasia which were treated by medial approach. None of the cases had any evidence of avascular necrosis of the femoral head and thickness of acetabular cartilage could be measured on hip artrographies for all cases. The difference of osseous acetabular index and cartilaginous acetabular index measured on artrographies were accepted as acetabular cartilage thickness. Hips of the children who were younger than 12 months during the operation were classified as group I and hip of the children who were between 12 and 18 months old during the operation as group II. Osseous asetabular index measured during the first and third years after operation and sharp angle measured during the last control were compare statistically.

Results: Mean follow up was 72 months (R: 47-135 months). Acetabular cartilage thicknesses were not statistically different in Group I and Group II. Mean acetabular cartilage thickness was $19.62^{\circ}$ and $19.77^{\circ}$ in group I and II respectively. However, OAI values measured at the end of the first and third postoperative years were significantly different. Sharp angle values of both groups measured during their last controls were statistically similar.
Conclusions: The thickness of acetabular cartilage should not affect development of acetabulum and prognosis of the hip. However, there was a relationship between age of reduction and acetabular development for the first 3 years. As a result, we conclude that in early reduced hips acetabulum would continue to develop as functional congruency maintained without any complication occurrence and secondary reconstructive osseous procedures should be delayed during this period as we mentioned before.

Significance: The thickness of acetabular cartilage should not affect of acetabular development.

\section{9}

\section{EVALUATION OF ISOKINETIC MUSCLE STRENGTH MEASUREMENTS IN PATIENTS WITH UNILATERAL DEVELOPMENTAL DYSPLASIA OF THE HIP TREATED WITH ONE-STAGE COMBINED PROCEDURE}

\author{
S. Yilmaz, A. Bicimoglu, E. Aksahin, H. Muratli, H. Y. Yuksel, \\ L. Celebi, M. Ersoz
}

Purpose: The patients treated with one-stage combined operations after walking age for developmental dysplasia of the hip (DDH), and whose follow-up revealed both clinical and radiological complete healing underwent flexor and extensor isokinetic muscle strength (IMS) measurements of the hip and results were evaluated in comparison with the contralateral hips.

Methods: A total of 22 patients with unilateral DDH and treated with one-stage combined operations after walking age were included in the study. In their last follow-up visit, all patients were functionally excellent in accordance with the Barrett's Modified McKay Criteria and according to the Severin's Classification for radiological grading of the hip all cases were type I. IMS of hip flexors and extensors were tested by Biodex 3 Pro isokinetic test device at 120 and $240^{\circ} / \mathrm{sc}$. In all patients, peak torque (PT), peak torque angle (PTA), total work (TW), and average power (AP) values of operated and non-operated hips were measured at both angular velocities and recorded separately for flexors and extensors. For comparative evaluation, values of the operated and nonoperated hips were used for determining the differences in IMS (DIMS), total work (DTW), and average power (DAP). In statistical assessment; Student's $t$ test, paired $t$ test, and Spearman's Rank correlation analysis were used. All significance levels were set at $\mathrm{p} \leq 0.05$.

Results: The mean age of patients were $12.8 \pm 2,9$ (9-18) years old. At the last control visit, the mean value of follow-up periods were $112.6 \pm 32,0$ (68-159) months. Parameters like age, age at the time of operation, and the length of postoperative follow-up period showed no statistical relation with IMS measurements $(P>0.05)$. For flexors, TW was lower at the operated hip when compared with the non-operated hip at 120 and $240^{\circ} / \mathrm{sc}(P$ 0,001 and $\mathrm{p}=0,002$, respectively). AP was lower at the operated hip at 120 and $240^{\circ} / \mathrm{sc}(P=0,011$ and $\mathrm{p}=0,003$, respectively). PT was lower at the operated hip $(22,5 \pm 11,3)$ when compared with the non-operated hip $(27,1 \pm 12,1)$ only at $120^{\circ} / \mathrm{sc}$ $(P=0,001)$. For extensor muscles, PT, TW, AP, and PTA showed no statistically significant difference $(P>0.05)$. For flexors, the DIMS between operated and non-operated hips at 120 and $240 \%$ sc were measured as $-15,3 \pm 22,2 \%$ (median; $-14,4$ ) and $-8,0 \pm 21,4 \%$ (median $-2,5)$, respectively.

Conclusions: In operated DDH patients, IMS measurements revealed that the flexor muscle strength of the operated hip was still 
weaker than the non-operated hip. At $120^{\circ} / \mathrm{sc}$, which represented evaluation against higher resistance, DIMS, DWF, and DAP were higher when compared with $240^{\circ} /$ sc.

Significance: The patients treated with one-stage combined procedure that had functional and radiological excellent results had hip flexors weak in activities like sports, which require more resistance.

\section{0}

\section{THE MRI EVALUATION OF ILIOPSOAS AND OTHER HIP MUSCLES IN PATIENTS WITH UNILATERAL DDH TREATED WITH ONE-STAGE COMBINED PROCEDURE}

\author{
H.Y. Yuksel, A. Bicimoglu, E. Aksahin, H. Muratli, L. Celebi, \\ S. Duran, S. Yilmaz
}

Purpose: The iliopsoas and other flexor-extensor muscles in operated and contralateral hips were evaluated comparatively by magnetic resonance imaging (MRI) in patients with developmental dysplasia of the hip (DDH).

Methods: A total of 22 patients with unilateral DDH after the walking age and treated with one-stage combined surgery were analyzed. All patients were operated by the same surgeon with complete tenotomy of iliopsoas muscle hindering open reduction. All patients had functionally excellent results in accordance with the Barrett's Modified McKay Criteria in their last follow-up visits and according to Severin's classification all cases were type 1. The imaging was performed by 1,5 T GE Excite MRI device at the supine position, without contrast material and sedation. The sagittal sections for iliopsoas muscle and T2-W FSE axial images for flexor and extensor muscle groups were used. The operated and contralateral sides were compared. Student's t test, paired t test, and Spearman's Rank correlation analysis were used for statistical assessment. All significance levels were set at $p$ $\leq 0.05$.

Results: The mean age was $12.8 \pm 2.9(9-18)$ years old. The mean postoperative follow-up period was $112.6 \pm 32,0$ (68-159) months. The reattachment of the iliopsoas to trochanter minor was observed in 7 patients, with no significance in terms of age, postoperative follow-up period, and the duration of postoperative period $(P>0,05)$. The atrophy in the operated side was significant in the length of iliopsoas muscle section area $(P=0.0001)$; and the section areas of rectus femoris $(P=0,002)$, tensor fascia lata $(P=0.0001)$, and gluteus maximus $(P=0.0001)$. No significance was detected in sartorius muscle section area $(P=0,886)$. However, unlike other muscles; the ratio of operated versus contralateral side mean muscle section areas was above 1 $(1.1 \pm 0,3)$ for the sartorius muscle. Iliopsoas muscle reattachment was not significant for ratios of the other muscles' operated versus contralateral side muscle section areas $(P>0.05)$. The atrophy was significant for the second $(P=0.03)$ and the third $(P=0,022)$ section's diameter ratios in the non-reattachment versus reattachment group for the iliopsoas muscle.

Conclusions: Following complete iliopsoas tenotomy, the expected compensatory hypertrophy in other flexor hip muscles was not detected. At the operated side, all evaluated muscles were atrophic except for the sartorius muscle. The atrophy of iliopsoas muscle was significant for the operated hip with non-reattachment to insertion site versus reattachment group.

Significance: The reattachment of the iliopsoas muscle to trochanter minor after complete tenotomy was observed in $32 \%$ of patients. Even at this nearly 10 years of follow-up period, patients treated with one-stage combined procedure had atrophy at hip flexor (except the sartorius) and extensor muscles.

\section{1}

\section{CHILDREN TARSAL COALITIONS: RESULTS OF RESECTION-FAT INTERPOSITION}

\author{
C. Bonnard, B. de Courtivron, C. Coulibali, F. Bergerault, \\ L. le Nail, M. Planchenault
}

Purpose: Tarsal coalitions in children are responsible of painful and rigid foot. Surgical treatment is often required. A conservative solution is resection with fat interposition witch is an alternative to arthrodesis and its complications. Results seem to be better if resection is made before 13 years. The purpose of this study was to evaluate the clinical results of resection-fat interposition and determinate if the age of resection, the sex or the localisation of the coalition had an impact on the final result.

Methods: 25 children with tarsal coalition were treated surgically by coalition resection and fat interposition between 1992 and 2006 (13 girls and 12 boys witch represents 32 feet). They were reviewed with a mean follow up of 41,5 months (14-126). The average age of resection was 12.5 years $(10,8-17,8)$ for the girls and 11,8 years $(5,2-18,2)$ for the boys. Clinical evaluation before and after operation was made with Comfort (1) scale (pain, activities, subtalar joint mobility). All patients had standard radiographs and scans for those who had talocalcanear coalition before resection and at follow up.

Results: We found 27 excellent results, 3 goods, 1 fair and 1 bad. With talocalcanear coalition, we found 15 excellent results and 1 good. With calcaneonavicular coalition, there were 10 excellent, 1 good and 1 fair result. At last, we had 2 excellent results with calaneocuboidian coalition and 1 good result with talonavicular coalition. There was no recurrence or secondary arthrodesis in our series. Moreover, no significant difference relationship was found between result and age of resection $(P=0.24)$, sex $(P=0,27)$, localization of coalition $(P$ $0.6)$ and size for the subtalar ones $(P=0.4)$.

Conclusions: Orthosis treatment of symptomatic tarsal coalition may be a waiting solution but resection in youngest child give better result.

Resection-fat interposition gives good results in child without recurrence. As a consequence, there is no indication for primary arthrodesis for us, even for talocalcanear coalition as some authors had written. Arthrodesis may be indicated in case of failure of resection.

Significance: We found no significance difference relationship between result and age or sex even with age corrected probably because our cohort of patient was all teen aged. It was the same for subtalar coalition size witch was mainly around $33 \%$ of the total joint.

\section{2}

\section{ARTHRODESIS TALO NAVICULARIS IN PATIENTS WITH NEUROLOGICAL DISORDERS AND FLAT FOOT DEFORMITY}

\section{G. de Coulon, A. Kaelin, D. Ceroni, V. de Rosa}

Purpose: Several techniques have already been described to solve the problem of flat foot deformity in children with neurological 
disorders (calcaneal lengthening, subtalar arthrodesis). These result sometimes in over or under correction of the deformity. The purpose of the study is to evaluate the effectiveness of the talo-navicular arthrodeses in the treatment of planovalgus foot deformity in non-ambulatory children with neurological disorders.

Methods: Methods and results: we retrospectively evaluated 16 cases of arthrodesis of the talus navicularis in 10 patients with neurological disorders.

Results: There were 5 boys and 5 girls. They exhibited the following diagnoses: Neurological syndromes: 4, Myopathy: 1, Diplegia: 4, Dystonia: 1. Average age at surgery was 11,2 years (7-19 years old) and the last follow up was performed at 3.8 years on average (range 1-9 years). Three feet were painful preoperatively and none after last follow up. Six patients were able to walk longer distance after surgery, the other 4 showed no changes. Applying the score for clinical outcome used by Won Yoo(1), 14 feet had a satisfactory result and two were unsatisfactory. Weight bearing radiographs measuring preoperative talo-1st metatarsal front and lateral view, talo-horizontal and talo-calcaneo angles were compared with post operative and last follow up X-ray. The reduction of the hind foot achieved post operatively was maintained at last follow up.

\begin{tabular}{llrl}
\hline & Pre op & Post op & Last follow up \\
\hline Talo-1st meta front view & 21.9 & 1.55 & 2.83 \\
Talo-1st meta lateral view & 42.9 & 7.84 & 5.16 \\
Talo horizontal & 51.18 & 22.18 & 19.56 \\
Talo-calcaneal lateral view & 52.56 & 33.31 & 28.2 \\
\hline
\end{tabular}

Two clinically unsatisfactory results were found: the first one was a patient with a hemiplegic disorder having a dystonic component were the evertors muscle were not balanced during surgery. Moreover, the operative fusion could not maintain the reduction as called for, thus fusion was not achieved in the desired position, requiring a later triple arthrodesis and tendon transfer. The second case was a Myopathic hypotonic patient with hyperlaxity that developed fore foot abduction with a stable hind foot.

Conclusions: This surgery seems to achieve a reliable hind foot fixation, without the complications of over and loosening correction seen with other techniques.

Significance: To our knowledge, results with talo-navicular arthrodesis in flat foot deformity in patients with neurological disabilities have not been described before.

\section{3}

\section{ASSESSMENT OF THE RESULT OF CLUB FOOT TREATMENT: A NEW QUANTIFIED KINEMATIC METHOD}

\section{W. P. Wicart, B. Maton, J. Dubousset, R. Seringe}

Purpose: The multiplicity of scores used in order to qualify the results of idiopathic clubfoot $(\mathrm{CF})$ treatment reveals the difficulty of this task. One of the sequelea of CF is triceps surae amyotrophy and insuffiency. This may induce an alteration of equilibrium control during swing phase of gait when the support foot is a $\mathrm{CF}$, particularly at heel contact (HC) which is the instant of maximal instability. It has been shown than vertical acceleration of the centre of gravity at $\mathrm{HC}(\mathrm{Z}$ 'HC) is an index of the capacity to control equilibrium, which depends on Triceps surae. Its positivity reflects an efficient control with absorption at $\mathrm{HC}$ and its negativity highlights an unrestrained fall of the centre of gravity. The goal of this paper is to qualify the usefulness of this parameter to assess the result of $\mathrm{CF}$ treatment.

Methods: Ten children with unilateral CF treated conservatively and 10 healthy children participated in the experiments. Mean age was 10.5 years old $(8.8-11.9)$. The values of Z'HC, centre of gravity forward velocity (V) and step length were calculated during gait.

Results: Positive Z"HC was found in $85 \%$ of healthy children trials. Positive Z"HC occurred in only $71 \%$ of children with $\mathrm{CF}$ trials when the support foot was the normal foot and 59\% of trials when the support foot was the CF. The rate of positive $\mathrm{Z}^{\prime \prime} \mathrm{HC}$ for children with $\mathrm{CF}$ differed from that of healthy children. It did not differ in the group of children with unilateral $\mathrm{CF}$, depending on the character pathological or not of the support foot. A positive correlation was found between $\mathrm{Z}$ "HC and V for healthy children, but was not observed in the group of children with unilateral $\mathrm{CF}$ whether the support foot was the normal or pathological one. Conclusions: The rate of positive $\mathrm{Z}^{\prime \prime} \mathrm{HC}$ was lower for children with CF. In healthy children group Z"HC increased proportionally to $\mathrm{V}$. This highlights a physiologic adaptation to a higher instability induced by longer step performed in order to increase $\mathrm{V}$. This correlation was not observed for children with unilateral $\mathrm{CF}$, when the support foot was the clubfoot. Triceps surae insufficiency may be responsible for the inability to control equilibrium when the support foot is the CF. Less expected was the absence of difference in the group of children with unilateral $\mathrm{CF}$, whether the support foot was the $\mathrm{CF}$ or the normal one. A hypothesis may be that the brain gives greater importance to symmetry than to the optimal use of the muscular system.

Significance: These data, inaccessible to clinical observation, highlight the effects of $\mathrm{CF}$ on equilibrium control. They provide an objective and quantitative evaluation of function and treatments result (rate of positive $Z^{\prime \prime} \mathrm{HC}$, slope of the correlation between $Z^{\prime \prime} \mathrm{HC}$ and V). This study describes original data about locomotor control alteration induced by a CF. The phenomenon of symmetry had never been described.

\section{4}

\section{LEG MUSCLE ATROPHY IN IDIOPATHIC CONGENITAL CLUBFOOT: IS IT PRIMITIVE OR ACQUIRED?}

\author{
F. De Maio, A. Orefice, E. Ippolito, F. Mancini, V. Potenza
}

Purpose: To investigate whether atrophy of the leg muscles present in congenital clubfoot (CCF) is primitive or secondary to the management of the deformity.

Methods: MRI of both legs was taken in: (a) 8 newborns with CCF before starting treatment (age range 1-2 weeks); (b) 8 infants who had Ponseti treatment, 2 months after Achilles tendon subcutaneous tenotomy (age range 4-5 months; 4-5 plaster casts applied); (c) 8 adults who had been corrected by manipulation, plaster cast and bracing, according to Ponseti, and limited posterior release (age range 19-22 years). All the patients had unilateral CCF. Muscles were measured on transverse MRI sections of the leg taken at the level of the maximum calf circumference, using a computer program (AutoCad2002LT). The same program was used to measure leg muscles on the histological sections of two fetuses with unilateral CCF aborted at 13 and 17 weeks of gestation. 
Results: The ratio between the muscle size measurement of the normal leg and that of the leg on the clubfoot side was very similar from fetal life to adulthood. No statistically significant difference was found between leg muscle atrophy in fetuses and untreated newborns and leg muscle atrophy in treated infants and adults.

Conclusions: In CCF, atrophy of the leg muscles has been described in several pathologic studies as well as in various clinical studies, but most authors still believes that atrophy Is secondary to prolonged immobilization in a plaster cast and brace as well as to surgical treatment. Our study shows that CCF treatment does not cause leg muscle atrophy, which appears to be a primitive pathologic component, already present during the early stages of fetal CCF development.

Significance: In CCF, leg muscle atrophy is a primitive pathologic condition, and it might play a role in the pathogenesis of the deformity.

\section{5}

\section{PHASES IN GAP HEALING FOLLOWING PONSETI TYPE ACHILLES TENOTOMIES - AN ULTRASONOGRAPHIC STUDY}

\section{R. Kanwar, H. Prem, K. Johnson}

Purpose: To assess the healing pattern of Achilles tendons across the gap created by a percutaneous tenotomy and maintained by cast in club feet.

Methods: 21 tenotomies in 16 patients (age range 12 weeks36 months) were monitored with dynamic and static ultrasonographic studies Ultrasounds were performed before and immediately after tenotomy and at approximately 3, 6 and 12 weeks post tenotomy. Cast removal was done at 3 weeks. Two patients above age of two were casted for 6 weeks.

Results: The healing pattern went through different phases although they were not distinctively exclusive from each other and did show considerable overlap. First phase showed formation of a bulbous mass with some continuity of scar tissue across tendon gap. The transition zone between new fiber and the original tend quite distinct. However dynamic ultrasound showed the Achilles tendon moved as a single unit. Second phase showed fibers resembling normal tendon crossing the gap and reduction of bulbous mass the transition zone was still discernible. Final stage demonstrated more homogenous fibers of Achilles tendon with an indistinct transition zone. Two older children showed a distinctly longer process of healing. One child showed an irregular mass of fluid and soft tissue structures in the gap at 6 weeks The other child demonstrated a relative reduction in the proportion of tendon fibers across the gap At 12 weeks there was evidence of continuation of tendon fibers, but transition zone partly visible. Conclusions: Ours study demonstrates when cast immobilisation is discontinued; the tendon is in mid phase of healing. It is safe to consider percutaneous tenotomy in children up to 3 years of ages provided the period of immobilization extended.

Significance: When cast immobilisation is discontinued there may be a positive effect on continued improvement in dorsiflexion while using boots and bars.

\section{6}

\section{THE EFFECTIVENESS OF PONSETI METHOD AFTER FAILED ORTHOPEDIC TREATMENT}

Purpose: The aim of this study is to evaluate the effectiveness of the Ponseti method in children with idiopathic clubfeet presenting after 3 months initially unsuccessfully treated by other conservative methods

Methods: We report 94 clubfeet of 64 patients, treated in two institutions between 1997 and 2004 with the Ponseti method. All of them had previous treatment with casting for a minimum of 3 months and some of them had received other minimum procedures ( 5 tenotomy and 4 open Achilles lengthening). The original orthopedists of 34 patients advised posteromedial release. 12 clubfeet could be described as complex. Patients who had neuromuscular and syndromic clubfeet or previous extensive surgery were excluded.

Results: Mean follow-up was 65 months (range115-39 m). We needed a medium of 3,6 casts (from 1 to 8 ) per clubfoot to achieve correction. $86 \%$ required a percutaneous tenotomy of the Achilles tendon. Relapse rate was 13\% (12 feet in 8 patients). We treated 3 relapses by restarting the use of orthosis, 2 with recasting, 7 with anterior tibial tendon transfer.We have compared this results with other similar series. We did not need to perform any posteromedial release to achieve correction.

Conclusions: Previous unsuccessful treatment attempts by other conservative methods did not adversely affect our results. Our results show that the Ponseti technique is reproducible and effective in children from 3 months $\backslash$ to 30 months. We have obtained similar results to other groups that have studied the effectiveness of Ponseti method in older children. It can also produce good correction in children presenting with complex deformities. Extensive joint releases can be avoided in such cases. Significance: Most pediatric orthopedists think that successful clubfoot casting depends on treatment started immediately after birth and in case of failed. Orthopaedic treatment they only can be solved by extensive joint surgeries (posterior or posteromedial release).Our series that analyze 94 previous treated clubfeet has obtained similar results to other consecutive series of Ponseti technique and with the use of Ponseti protocol all our cases have been solved without extensive surgery.

\section{7}

\section{EARLY RESULTS OF THE PONSETI METHOD FOR TREATMENT OF ARTHROGRYPOTIC CLUBFOOT}

\section{S. Boehm, F. Alaee, M. Dobbs, M. F. Sinclair, N. Limpaphayom}

Purpose: Clubfoot occurs in approximately 1 in 1,000 live births and is one of the most common congenital birth defects. Although there are multiple reports of successful treatment of idiopathic clubfeet with the Ponseti method, the use of this treatment in nonidiopathic clubfeet has not been reported. This purpose of this study was to evaluate early results of the Ponseti method for the treatment of clubfeet associated with distal arthrogryposis.

Methods: Twelve consecutive infants with clubfoot ( 24 clubfeet) associated with distal arthrogryposis were treated with the Ponseti method and retrospectively reviewed. Four patients had casting treatment prior to referral. The severity of the foot deformity was classified according to the grading system of Dimeglio. The number of casts required to achieve correction was compared to published data for idiopathic clubfeet. Any recurrent clubfoot deformities or complications during treatment were recorded. All patients were followed for a minimum of 2 years.

Results: The clubfeet of all twelve patients (24 clubfeet) were graded as Dimeglio grade IV. Initial correction was achieved in all 
patients with a mean of $6.75 \pm 0.86$ casts (range, two to ten casts), which was significantly more compared to the number needed in a published cohort of idiopathic clubfeet treated with the Ponseti method $(P<0.003)$. Three patients (six clubfeet) had a relapse after initial successful treatment. All relapses were related to non-compliance with brace wear. No relapses occurred in the cohort of patients who were initially treated with the new dynamic foot abduction orthosis (eight patients). Two of the three patients with clubfoot relapse were successfully treated with repeat castings and/or tenotomy; the remaining patient (two clubfeet) was treated with extensive soft-tissue release surgery.

Conclusions: Our data supports the use of the Ponseti method in patients with distal arthrogryposis based on success rates approaching that for idiopathic clubfoot. Maintaining correction is perhaps the most difficult aspect of management. Parental teaching and early attention to brace complications are helpful techniques to improve parental compliance.

Significance: Treatment of relapses to date has been with repeat manipulations and castings as is the case for idiopathic clubfoot. Extensive soft-tissue release surgery for idiopathic clubfoot has been shown to have many negative long-term consequences in terms of foot pain, ankle and foot osteoarthritis, and quality of life. We anticipate that minimizing the amount of extensive softtissue release surgery for correction of arthrogrypotic clubfoot will lead to a more flexible foot with less long-term complications.

\section{Poster Presentations}

\section{8}

\section{AMBULATION IN CHILDREN WITH MYELOMENINGOCELE: NEUROLOGICAL, MUSCULOSKELETAL AND SOCIOECONOMIC ASPECTS}

\author{
A. Danielsson, Å. Bartonek, E. Levey, H. Saraste, K. McHale, \\ P. Sponseller
}

Purpose: Modern principles for treatment of patients with myelomeningocele include early closure of the neural tube defect, neurosurgical treatment of hydrocephalus and treatment aimed at minimizing contractures and joint dislocations. The aim is to achieve a better survival rate and a better quality of life in which the ambulatory function has a role. The focus for this study was to evaluate which factors, including the social environment, might affect the ability to ambulate in a child with paralysis due to myelomeningocele.

Methods: Forty patients (aged 4-16 years) with neurological deficit from myelomeningocele were examined in an unbiased followup. This included a physical examination using validated methods for ambulatory function and neuromuscular status, chart reviews and evaluation of radiographs in terms of hip dislocation and spine deformity. Socioeconomic characteristics of the family as well as need of educational support of the child were collected through parental interviews.

Results: $65 \%$ of the patients had quadriceps strength of 4-5 and $25 \%$ had spasticity around hip and/or knee joint muscles. Nine patients $(22 \%)$ had some degree of mental retardation. 21 children were ambulatory and 19 non-ambulatory. Existence of spasticity around knee or hip, contracture of resp. hip $>20^{\circ}$, knee $>20^{\circ}$ or foot $>15^{\circ}$, as well as hip dislocation or spinal deformity with Cobb angle above $30^{\circ}$ were significantly more frequently seen in the non-ambulatory group. $19 / 21(90 \%)$ of ambulatory patients had located hips, while $11 / 13 \quad(84 \%)$ patients with hip dislocation(s) were non-ambulatory, $\mathrm{p}=0.0028$. When controlling for each of all factors significantly associated to ambulation (incl. muscle function of lower limbs and strength of quadriceps) hip dislocation was still found to be significantly more frequent among non-ambulatory patients. Ninety-five per cent of the patients lived with one or both of their parents. In $32 / 40$ families at least one parent worked outside their homes. 18 patients $(47 \%)$ had no parent at home during daytime. The family situation, education of parents or whether one parent was at home during daytime did not affect ambulatory function.

Conclusions: Physical issues, specifically spasticity around the hip and/or knee joint, hip dislocation and scoliosis affected ambulation negatively. In contrast, social environment did not seem to affect the possibility to ambulate.

Significance: As physical issues were shown to affect ambulation, efforts in order to improve the patients physical capacity to ambulate, seem to be warranted.

\section{9}

\section{MANAGEMENT OF NEUROPATHIC ARTHROPATHY IN CHILDREN}

\section{G. A. Hosny}

Purpose: Neuropathic arthropathy is characterized by relatively painless swelling together with extensive damage in bones and joints.It is rarely seen in children and may be found in association with neurological disorders (Lokiec et al. 1998). Deformity and instability are the main problems encountered in these patients which has to be treated essentially by education and bracing. However, failure of conservative treatment in children may lead to marked restriction of activity and serious complications. We report our experience in treating seven patients after failure of conservative treatment.

Methods: From 2000 till 2006, 7 cases of neuropathic arthropathy of the ankle or the knee in children were referred to our center. There were 4 girls. The procedure was performed bilateral in 5 cases and unilateral in 2 case ( 9 ankles and 3 knees).The etiology was diabetes in 6 cases and congenital insensitivity to pain in one case. The age of patients ranged from 8 to 12 years old. The patients were referred after a period of conservative treatment ranging from 6 months to 18 months. The operative procedure for the Charcot ankles was application of circular fixator and alternating cycles on compression, distraction to gain fusion. For the case with bilateral Charcot knee and severe valgus deformity, we corrected the deformity gradually through 2 osteotomies in the lower femur and upper tibia. The patients were evaluated according to stability, progression of joint destruction, functional activity and satisfaction of the patients.

Results: The follow up period ranged from 9 months to 3 years 6 months. There were six good and one poor result. Time in the External fixator ranged from 4 to 6 months. Partial gradual recurrence of the deformity developed in one knee. Complications included: 1-Pin tract infection in all cases. 2-Common peroneal nerve palsy in one case.

Conclusions: Up to the best of our knowledge surgical managent of neuropathic arthropathy in children has not been previously reported. Gradual correction of deformities may delay progressive joint destruction. Bloodless arthrodesis is possible using circular fixators.

Significance: Treatment of neuropathic arthropathy of the ankle or the knee in children using external fixator is a valid option. 


\section{0}

\section{BACLOFEN PUMP IMPLANTATION AND SPINAL FUSIONS IN CHILDREN: DOES ORDER OR TIMING INFLUENCE COMPLICATIONS?}

\section{A. Borowski, A. Littleton, F. Miller, K. Dabney, S. Shah}

Purpose: A common treatment for spasticity in children with cerebral palsy is intrathecal baclofen therapy (ITB). This same population also has a high incidence of spinal deformities requiring posterior spinal fusion (PSF). The goal of this research is to report the technical considerations and complications associated with ITB in patients with PSF.

Methods: There are four groups: A, 26 patients with PSF prior ITB; B, 11 patients with PSF and ITB concurrently; C, 25 patients with PSF after ITB; and Control group: 103 patients with ITB only. Complications and infections were tabulated from retrospective chart review. Analysis of Variance (ANOVA) was used to compare the rate of complications and infections between groups. Patients with healed PSF who are to have ITB are positioned in side lying. The subperiosteal exposure of the fusion mass is preformed and a burr is used to open a hole in the fusion mass. The catheter is passed and secured. The bone hole is closed with Cranioplast or To-Seal. To implant a pump at the time of PSF, the intrathecal catheter is implanted prior to wound closure. The catheter is brought out through the paraspinal muscles, anchored to the fascia, then a two centimeter incision is made at mid-lateral abdomen and the catheter is pulled into this pocket rolled up and the wound is sutured. After wound closure the patient is turned supine and pump implanted. PSF in patients with a pump have the catheter exposed, cut and removed, and tucked into a lateral subcutaneous pocket. After the spine fusion the catheter is reinserted.

Results: The outcome by group showed: Group A with 5 catheter malfunctions, 2 infections at pump. Group B had 2 catheter malfunctions, 1 pump hypermobility, and 1 infection at spine. Group $\mathrm{C}$ had 3 catheter malfunctions, 1 infection at pump, and 1 infection at spine. Control group of 103 patients had 23 catheter malfunctions, 5 pump failures, 8 infections at the pump, and 1 infection at spine. ANOVA showed no difference in the rate of infection or device complications between any of the groups.

Conclusions: The rate of ITB therapy complications is not increased despite PSF in any order of the procedures. There are technical details in each situation that require attention.

Significance: By understanding the appropriate techniques ITB pumps can be implanted and managed with no increased complication rate before, after or during spinal fusion surgery.

\section{1}

\section{DEEP WOUND INFECTION AFTER SPINAL FUSION IN CEREBRAL PALSY, DOES ANTIBIOTIC-LOADED ALLOGRAFT MATTER?}

\section{F. Miller, A. Borowski, A. Littleton, B. Borkhuu, K. Dabney}

Purpose: Previous studies evaluating wound infection rates after spinal fusion surgery in children with cerebral palsy report a $4.1 \%$ deep wound infection rate. The concept of using antibiotic-loaded bone graft to provide local antibiotics has been explored in high risk patients, such as those with osteomyelitis. There have been no reports of using antibiotic-loaded bone graft prophylactically in spine surgery. Our purpose is to determine and compare the infection rate after spinal fusion with unit rod instrumentation and bone allograft with or without Gentamycin in children with cerebral palsy.

Methods: After IRB approval, the medical records of 220 children with cerebral palsy (CP) who had spinal fusion with unit rod instrumentation for a primary spinal deformity between January 2000 and December 2006 in single institution were retrospectively reviewed. We evaluated the incidence of postoperative wound infection in patients with antibiotic-loaded bone graft (AbBGF) and those without (BGF).

Results: One hundred fifty-four patients received AbBGF during spinal fusion surgery with 6 patients $(3.9 \%)$ complicated with a deep wound infection. Ten of the 66 patients $(15.2 \%)$ without antibiotic-loaded bone graft developed a deep wound infection. The difference between groups was statistically different $(P$ 0.003). The mean age at surgery, preoperative Cobb angle, correction rate, operative time, and estimated blood loss were not statistically different between the two groups $(P>0.05)$. The length of hospital stay was less in AbBGF group $(P<0.05)$.

Conclusions: The incidence of deep wound infection for children with $\mathrm{CP}$ undergoing spinal fusion decreased from 15 to $4 \%$ with the use of prophylactic antibiotics in the corticocancelluos allograft.

Significance: The incidence of deep wound infection after spinal fusion in 220 children with CP decreased from 15 to $4 \%$ with the use of prophylactic antibiotics in the corticocancellous allograft.

\section{2}

\section{SPINAL CORD CHANGES DURING SCOLIOSIS SURGERY}

J. Burgos, C. Barrios, G. De Blas, G. Pizà, I. Sanpera, O. Riquelme

Purpose: The spinal cord in scoliosis is placed on the concave side of the spine. Correction of the deformity has been said to modify position. The present study assesses the variation of position of the spinal cord after surgery.

Methods: The study was divided in two sections: (a) Clinical section: 6 girls with AIS (Adolescent Idiopatic scoliosis), average age 14.7, with a curve Lenke type 1 of curve average of $63^{\circ}$, were instrumented using pedicular screws and VCA correction technique, the apex was not instrumented. Pre and postoperative MRI was used to assess spinal cord position. (b) Experimental group: 6 industrial pigs with a experimentally produced scoliosis (by previous costo-transvesrectomy), with an average Cobb of $34^{\circ}$. The pig had a posterior approach with resection of posterior elements. The pedicles were instrumented and the VCA applied.

Results: The clinical group had a correction of the curve of $81 \%$. No changes in the distance pedicle-spinal cord, or pedicle-dura were observed between the pre and the postoperative MRI. In the experimental group no changes in the distance pedicle-dura were observed between the pre and post-reduction position.

Conclusions: Contrary to previous belief, scoliosis reduction does not change the position of the spinal cord. This finding will explain why the pedicular screws on the convexity are safer and with less complications than those on the concavity. This could also be an explanation for the different electrophysiological values of the screws in the convexity and concavity. Long term studies will be needed. 
Significance: This is a prospective clinical study, assessing the changes in position of the spinal cord, after clinical and experimental correction of spinal deformity. Evidence would suggest that screws on the convexity are safer than those in concavity.

\section{3}

\section{CORONAL PLANE RESULT OF SURGERY IN PATIENTS WITH SCOLIOSIS TYPE 5 AND 6 OF LENKE}

\section{B. Jasiewicz, D. Zarzycki, M. Tesiorowski, T. Potaczek, W. Kacki}

Purpose: Scoliosis as a triplanar deformity leads to some changes in trunk and disturbs its symmetry. Basic rules of surgery in scoliosis consist of: curve correction in frontal plane and normal sagittal alignment restoration. Goals of work: 1 . How changes the coronal plane alignment after surgery in idiopathic scoliosis, type 5 and 6 according to Lenke classification. 2. What are the factors that influence the end result?

Methods: Material consists of 52 patients. The mean age at the time of surgery was 16 years with the follow up time of 4 years. There were 29 patients in first group with Lenke type 5 and 23 patients in the second group with type 6 . The Cobb angle was in Lenke 5 group $52.50 \pm 5.9$ and in Lenke 6 group $-54.4 \mathrm{o} \pm 8.4 \mathrm{in}$ thoracic spine and $66.3 \mathrm{o} \pm 11.9$ in lumbar spine. The mean distance between $\mathrm{C} 7$ and central sacral line was $20.8 \mathrm{~mm} \pm 11.1$ in Lenke 5 group and $14.9 \mathrm{~mm} \pm 12.4$ in Lenke 6 group. Angle of the fourth lumbar vertebra was $24.9 \mathrm{o} \pm 5.4$ in first and $28.2 \mathrm{o} \pm 7.4$ in second groups. Trunk shift was greater in first group: $-14.5 \mathrm{~mm} \pm 10.7$ in comparison with $-7.8 \mathrm{~mm} \pm 9.5$ in the Lenke 6 group. All the patients underwent posterior fusion with derotational instrumentation with or without pedicle screws in lumbar spine. Radiological assessment was performed using postero-anterior and lateral radiograms. Own scale of treatment result evaluation was introduced.

Results: During control examination the curve angle was $19.7^{\circ} \pm 10.2$ in first group and in second thoracic angle was $27.3^{\circ} \pm 9.2$ and lumbar was $25.0^{\circ} \pm 9.6$. Final correction in lumbar spine was greater than in thoracic spine: $62.2 \%$ in comparison with $49.8 \%$. During follow-up, the mean distance between $\mathrm{C} 7$ and central sacral line was $9.5 \mathrm{~mm} \pm 6.4$ in first group and $7.9 \mathrm{~mm} \pm 6.1$ in second group. Angle of L4 was $9.2^{\circ} \pm 7.6$ and $8.7^{\circ} \pm 5.5$ respectively. Trunk shift diminished and during control examination equaled $-8 \mathrm{~mm} \pm 8.6$ and $-6 \mathrm{~mm} \pm 10.7$. Good results in frontal plane were noted in 20 cases $(69 \%)$ from Lenke 5 group and 15 cases $(65 \%)$ from Lenke 6 group. The presence of pedicle screws in lumbar spine was bound with significantly better end result $(P<0.005)$. Fusion up to L3 vertebra gave bad result in frontal plane. Fusion to L4 was connected with significantly better result when: lumbar Cobb angle $>60^{\circ}$; elongation lumbar angle $\geq 25^{\circ}$ and L4 angle $>10^{\circ}$. The end result depends on preoperative planning including Cobb angles, type of scoliosis, elongation lumbar angle nad L4 angle.

Conclusions: (1) Own method of describing result in coronal plane allows better assessment of coronal balance. (2) Final correction in lumbar spine is greater than in thoracic spine. (3) The end reslut depends on preoperative planning including Cobb angles, type of scoliosis, elongation lumbar angle and L4 angle.

Significance: (1) Intruducing an own method of describing the coronal balance after spine surgery. (2) Establishing preoperative risk factors of bad result.

\section{4}

\section{EFFECTIVENESS OF ADOLESCENT IDIOPATHIC SCOLIOSIS TREATMENT WITH A NON-RIGID BRACE-EARLY RESULTS}

\author{
T. Potaczek, B. Jasiewicz, E. Lipik, K. Lokas, M. Zarzycka, \\ W. Kacki
}

Purpose: Adolescent idiopathic scoliosis is a three-dimensional deformation of the spine, treatment depends on many factors, with curve magnitude (Cobb angle) and skeletal maturity (Risser sign) being the most important. In progressing curves $<40^{\circ}$ Cobb angle, bracing is recommended. SpineCor dynamic brace is a system of elastic bands designed to directly correct the spinal column deformity without restricting motion of the spine. Aim of this paper is to present early results of adolescent idiopathic scoliosis treatment with the SpineCor brace.

Methods: Inclusion criteria for brace application in our study group were presence of a progressing curve in a skeletally immature child and no previous treatment. The SpineCor brace was applied according to the principles, patients were supposed to wear the brace $20 \mathrm{~h}$ /day. Study group consisted of 42 patients, 36 girls, 6 boys. Mean age at brace application was 11,9 years. Mean follow-up was 11 months. We evaluated the curve magnitude before treatment and during follow-up. Study group was then divided in three subgroups depending on the curve size: under $25^{\circ}$ $(n=10)$, between $25^{\circ}$ and $40^{\circ}(n=25)$ and curves over $40^{\circ}$ $(n=7)$ Cobb angle. Results were classified as correction (decrease of curve size $\geq 5^{\circ}$ ), stabilization (curve change $\pm 5^{\circ}$ ) or progression (increase of curve size $\geq 5^{\circ}$ ). Mean initial curve size in the whole group was $33.1^{\circ}$ in the thoracic spine and $29,4^{\circ}$ in the lumbar spine.

Results: Mean curve size at final follow-up was $29,7^{\circ}$ in the thoracic spine and $26,5^{\circ}$ in the lumbar spine. 15 patients improved $(35.7 \%), 21$ had curve stabilization $(50 \%)$ and 6 progressed $(14,3 \%)$. Best results were achieved in curves lower than $25^{\circ} \mathrm{Cobb}$ angle $(P<0.05), 60 \%$ improved, $40 \%$ were stabilized and none progressed. In contrary the over $40^{\circ} \mathrm{Cobb}$ angle group only 1 patient improved and 4 progressed.

Conclusions: SpineCor brace seems to be a good alternative for rigid braces; especially in low curves it gives very good results. In curves greater than $40^{\circ}$ rarely improvement is observed. This type of brace is easily accepted by young patients. Further follow-up is needed to present long term results.

Significance: (1) Evaluation of non-rigid brace effectiveness. (2) It is a beginning of a bigger prospective study.

\section{5}

\section{THE MEASUREMENTS AND STANDARDIZATION OF HUMEROCONDYLAR ANGLE IN CHILDREN}

N. Simanovsky, N. Hiller, N. Simanovsky, R. Lamdan, S. Porat

Purpose: Supracondylar fractures of the humerus in children are very common. However, the literature regarding measurements of normal anatomical relationships of the distal humerus in sagital plane is sparse.

Methods: We reviewed radiographs of normal elbow joints in 142 children treated in our hospital over 2 years. No history of previous trauma of distal humerus was found. The children were separated into three age groups (under $5,5-10$, and $10-15$ years) 
and measurement of the humerocondylar angle (HCA) in sagittal plane was performed.

Results: The mean age of children in group 1 was 3 years one month, in group 2 was 7 years eight months, and 12 years in group3. There were 99 boys and 43 girls. The mean HCA was $41.6^{\circ}$ (range $30^{\circ}-70^{\circ}$ ). No statistically significant influence on HCA by age, gender or side was found. We found a small number of extreme variants in $\mathrm{HCA}$ (down to $30^{\circ}$ and up to $70^{\circ}$ ) in children without any history of previous trauma and having a normal range of elbow motion.

Conclusions: We found that HCA is close to the well-accepted figure of $40^{\circ}$. Interestingly, this value remains the same in all age groups. That means that the geometry of the distal humerus in sagittal plane is established very early during the growth and remains constant. Due to significant individual variations of HCA it alone can not be sufficient for final decisions in evaluation and treatment of supracondylar fractures.

Significance: First evidence based quantific evaluation of the elbow in sagittal plane that standardized measurements of humerocondylar angle in children.

\section{6}

\section{DIAGNOSTIC IMAGING OF THE KNEE IN CHILDREN WITH ARTHROGRYPOSIS AND KNEE EXTENSION OR HYPEREXTENSION CONTRACTURE}

\author{
A. Borowski, A. Littleton, L. Grissom, M. Donohoe, M. King, \\ S. JayaKumar
}

Purpose: Amyoplasia (type I), characterized by quadrimelic limb involvement, is one of the most common types of artrogryposis. In children with hyperextension or extension contracture of the knee, subluxation or dislocation of the knee joint with an associated subluxation or dislocation of the patella is frequently seen. Patellar malpositioning may be secondary to congenital hypoplasia of the patellar groove of the femur and dysplasia of the patello-femoral joint cause patellar instability. A contracted quadriceps tendon and illiotibial band as seen in extension contracture of the knee in arthrogryposis also contributes to Patella alta and lateral subluxation of the patella. The aim of our study is to determine the position of the patella in children with quadrimelic arthrogryposis and knee extension contracture.

Methods: The inclusion criteria for this study were: (1) A patient should have arthrogryposis multiplex congenita, group I. (2) Have a knee extension contracture from birth. (3) Follow up from birth or early childhood. (4) At least yearly physical examinations. (5) Have a knee ultrasonography, or magnetics resonance imaging. Patellar position was determined and the cartilaginous femoral sulcus angle (CSA) as well as the osseous femoral sulcus angle (OSA) was measured.

Results: Clinically, in none of these patients could we palpate the patella prior to surgery. Patella was displaced superio-laterally in $16 / 16$ ultrasonography revealed knees. The average CSA was $161^{\circ}$ (normal $146^{\circ} \pm 3.7^{\circ}$ ), and ranged from 164 to $156^{\circ}$. The average OSA was $168^{\circ}$, range from 158 to 170 (normal values range from 180 to 165 by the age of 5). An MRI also shoved the patella to be displaced.

Conclusions: All patients in our series with extension contracture of the knee and type I artrogryposis had a patella that was superior and lateral to the patellar groove. We found that, quadricepsplasty and relocation of the patella improved knee flexion. All patients regained active knee extension in 6 months post surgery. We prefer to perform quadricepsplasty around
3 years of age, so that the patients could cooperate with splinting and physical therapy.

Significance: Patients in our study presents extension contracture of the knee and type I artrogryposis with patella that was superior and lateral to the patellar groove with dysplasia of femoral sulcus.

\section{7}

\section{SHELF ACETABULOPLASTY USING INNER TABLE ILIAC BONE GRAFT}

\section{W. G. Mackenzie, A. Littleton, D. Thawrani, P. Woratanarat}

Purpose: A new technique of shelf acetabuloplasty has been devised and implemented for the treatment of inadequate acetabular coverage. Several techniques of shelf acetabuloplasty have been described. The use of inner table iliac graft has several advantages in that a large, continuous graft with a cortical surface can be harvested, the graft has a natural dome shape, and there is no need to elevate the abductors.

Methods: Shelf acetabuloplasty was performed on 20 hips in 16 patients, using inner table iliac wing graft, between 1995 and 2006. Four patients with Morquio syndrome underwent bilateral shelf procedures and 12 patients with Legg-Clave-Perthes disease (LCPD) underwent a unilateral procedure. The average age at the time of surgery was 9.8 years (range, 5.4-12.9) with an average follow up of 42.7 months (range, 9.2-137.2 months). Hips were approached through a standard Modified Smith Peterson approach using a bikini incision. The graft for the shelf was harvested from the inner table iliac wing. Radiographic evaluation included preoperative, postoperative and follow up assessments of standing AP radiographs.

Results: All patients showed bony union at the osteotomy site. No adverse effects with respect to range of motion, pain, muscle weakness and stability as compared to the pre-operative status of the patient were observed. Improvement was seen in the angle of sharp (from $47^{\circ}$ to $35^{\circ}$ ), acetabular depth (from 24 to $29 \%$ ), percentage of femoral head coverage (from 68 to 103\%) and center edge (CE) angle (from $14^{\circ}$ to $49^{\circ}$ ). Computerized Topographic images revealed satisfactory global coverage of the femoral head and good graft incorporation.

Conclusions: We describe the technique and present the results of patients who underwent shelf acetabuloplasty utilizing inner table iliac bone graft. The inner table iliac wing is an ideal source of bone graft for shelf acetabuloplasty due to its intrinsic shape, cortical surface, minimal secondary impact at the harvest site, and abundant supply.

Significance: The inner table iliac wing is an ideal source of bone graft for shelf acetabuloplasty due to its intrinsic shape, cortical surface, minimal secondary impact at the harvest site, and abundant supply.

\section{8}

\section{LONG TERM ASSESSMENT OF UNILATERAL DEVELOPMENTAL DYSPLASIA OF THE HIP AFTER THE WALKING AGE USING QUANTITATIVE GAIT ANALYSIS: KINEMATIC HIP DATA}

H. Y. Yuksel, A. Bicimoglu, E. Komurcu, G. Yavuzer, H. Muratli, L. Celebi, S. Yilmaz 
Purpose: To quantify the gait characteristics of children with unilateral developmental dysplasia of the hip (DDH) after the walking age; treated by one-stage combined procedure in terms of kinematic characteristics using quantitative gait analysis.

Methods: Fifteen patients with unilateral DDH after the walking age; treated with one-stage combined procedure were included in this study. The kinematic parameters of operated and non-operated hips were evaluated by quantitative gait analysis. Quantitative gait data was collected with Vicon 370 (Oxford Metrics, Oxford, UK). Two force plates (Bertec, Colombus, Ohio, USA) were used for kinetic analysis. All time-distance (walking velocity, cadence, step time, step length, double support time), kinematic (joint rotation angles of pelvis, hip, knee and ankle in sagittal, coronal and transverse planes) and kinetic (ground reaction forces, moments and powers of hip, knee and ankle) data were processed using Vicon Clinical Manager software package. A typical walk was selected for each limb on the basis of consistency of temporo-spatial characteristics for assessments. Collected data were compared to our normal database for children. The Wilcoxon test was used for statistical analysis. All significance levels were set at $\mathrm{p} \leq 0.05$.

Results: The mean ages of patients were 12.3 (8-17) years old. The mean follow-up duration was 8.6 years (range 6-13 years). In all patients, the range of motion was complete. When the time-distance parameters (walking velocity, cadence, step time, step length, double support time) after the gait analysis were evaluated, no statistical difference was detected. When the kinematic hip data were evaluated, no statistical difference was detected between the maximum extension value in the stance phase $(P=0.733)$ and the maximum flexion value in the swing phase $(P=0.730)$ of normal and operated hips in the sagittal plane. A statistically significant difference was detected between the maximum adduction values $(P=0.002)$ of the normal and operated hips in the coronal plane. When related arithmetic means were analyzed, hip adduction of the non-operated hip was significantly increased with regard to the operated hip $(13.2 \pm 4.2>2.5 \pm 5.8)$.

Conclusions: Patients keep their non-operated hip at adduction during stance and swing phases. This results from avoiding their operated hip. The reason for the maintenance of this adduction was believed to depend on the disturbance in the lever arm.

Significance: The quantitative gait analysis revealed the current disturbance in the kinematic hip data belonging to patients treated with one-stage combined procedure who had excellent functional and radiological results.

\section{9}

\section{LEGG-PERTHES-CALVÉ DISEASE: DO GIRLS REALLY HAVE A POOR PROGNOSIS?}

\section{F. Canavese, A. Dimeglio, M. Ali}

Purpose: Girls tend to develop Legg-Perthes-Calvé disease (LPC) four times less than boys and seem to have a poorer prognosis: this is felt to be because girls reach skeletal maturity sooner than boys and have a shorter femoral head remodeling period. Other hypotheses have suggested that the late onset of the disease and/or the higher frequency of severe forms could be responsible for the poor prognosis. The aim of this study is to evaluate the evolution and the effects of treatment in girls with LPC that have reached skeletal maturity.
Methods: A retrospective review of the records and roentgenograms of all patients was performed of all patients with LPC who had been seen at our institutions during the period 1970-2005. The hips were graded according to the Catterall's classification, the lateral pillar classification, and the Salter-Thompson's classification. Mild forms were not actively treated. Patients with severe forms received active treatment: orthopedic traction, wheelchair with abductor device and/or surgical intervention. When a lateral extrusion of the femoral head was present our preferred surgical treatment consisted of Innominate or Triple Osteotomy. The outcome at skeletal maturity was evaluated according to Stulberg's classification. Statistical ( $\chi^{2}$ test) analysis was performed. Results: This is a homogenous series of female patients with LPC treated by the same team, following the same indications and procedures. 505 hips were identified: 244 hips developed the disease before the age of 6,201 between 6 and 9, 60 over the age of 9 . $21.6 \%$ of patients were girls. 72 out of 109 girls ( 79 hips) had reached skeletal maturity and were included in the study.40 hips developed the disease before the age of 6,28 between 6 and 9 years of age, and 11 after 9 years of age. The average age at the onset of symptoms was 6.07 years (range: $2.5-12$ years). 29 severe forms underwent conservative treatment and 32 had surgical treatments. Overall results showed $81,1 \%$ of Stulberg I-II in girls with less than 6 years of age, $77.7 \%$ in girls between 6 and 9 , and $45.5 \%$ in girls over age 9.Stulberg III-V were $18.9,22.3$, and $55.5 \%$ in the respective age groups.

Conclusions: The disease is more common in boys $(\mathrm{M}: \mathrm{F}=4.7: 1)$ and does not spare younger children as in both sexes $50 \%$ of cases develop before age 6 years and severe forms represent $2 / 3$ of cases in all age groups. Mild forms have a good prognosis in both sexes but if the age at onset is over age 9 years the outcome is frequently unsatisfactory. The outcome of severe forms is related to patient age at time of onset and amount of femoral head involvement: older age and whole femoral head involvement are poor prognostic factors. Surgery should be reserved to those hips that tend to subluxate and Salter or Triple Osteotomy may improve the femoral head containment and outcome in girls with later onset of the disease. The gender does not influence the outcome of the disease.

\section{0}

\section{SURGICAL TREATMENT OF HEALED PERTHES DISEASE IN THE ADOLESCENT: RECENT EXPERIENCE WITH BOTH INTRAARTICULAR AND EXTRAARTICULAR PROCEDURES TO TREAT FEMORO-ACETABULAR IMPINGEMENT}

\author{
M. B. Millis, J. Kasser, M.D., Y. Kim
}

Purpose: To report our experience with advanced imaging to improve the understanding of femoroacetabular impingement in symptomatic hips with post-Perthes deformity; and to report our early and middle-term experience with combinations of intraarticular and extraartcular surgery to treat the lesions thus diagnosed.

Methods: Plain radiography was supplemented with both MR imaging (intravenous or intraarticular gadolinium enhancement) using radial sequences; and with CT scanning (3 dimensional reconstructions) to optimize the understanding of the abnormal mechanics in symptomatic hips with post-Perthes deformity. This allowed rational selection of intraarticular and/or extraarticular corrective surgery based on the contemporary concepts of femoroacetabular impingement. 
Results: More than 50 symptomatic hips with post-Perthes deformity have been analyzed preoperatively with advanced CT and MR techniquues to optimize treatment selection. Surgical treatment of this group has included surgical dislocation with proximal femoral ostochondroplasty, acetabular rim osteochondroplasty, microfracture, labral debridement(either open or arthroscopic), labral takedown and refixation(open), intertrochanteric osteotomy, relative femoral neck lengthening, shelf osteoplasty, Chiari osteotomy, and periacetabular osteotomy. In our $6+$ year experience with the surgical dislocation approach, WOMAC scores reflect improved results over prior purely extraarticular methods.

Conclusions: Advanced imaging allows improved assessment of the intraarticular deformity in post-Perthes hips. Employing the improved knowledge of the deformity with contemporary powerful corrective intraarticular surgery seems to give improved clinical results over historical methods.

Significance: Post-Perthes deformity is a common cause of arthrosis in young active patients. Delaying or eliminating the need for arthroplasty by timely treatment of the symptomatic adolescent should be an important concern of the pediatric orthopedist.

\section{1}

\section{ARTHRODIASTASIS OF THE HIP WITHOUT SOFT TISSUE RELEASE}

\section{G.A. Hosny, K. Eldeeb, M. Fadel}

Purpose: Arthrodiastasis has been described as a valid option for these cases with Perthes disease where poor results are expected from other treatment modalities. Recently, there were many reports stating promising results. However, soft tissue release was performed in most cases. In this study we report our results after application of athrodistasis without soft tissue release.

Methods: From 1994 till 2006, 29 cases with Perthes disease were treated with arthrodiastasis in our institution. 3 cases were excluded due to inadequate follow up data. All cases were older than 7 years old.The age of patients ranged from 7 to 14 years. There were 18 males.13 patients had previous operations [range 1-3].All patients were assessed clinically and radiogrphicaly before and after the operation. Clinically,the presence and degree of pain,gait ,ROM, satisfaction of the patients were recorded. The patients were classified according to Herring lateral pillar classification and Catterall head at risk signs. Ilizarov fixator was applied to the supra-acetabular area and the femur and gradual distraction usually starts 3 days afterwards without any soft tissue release. The average fixation period was 3 months $[2-4.5 \mathrm{~m}]$

Results: 22 patients showed improved clinical results with 7 excellent and 18 good outcome. There was improvement of the degree of pain in all cases except 2. Complication include pin tract infection specially in the acetabular half pins, knee stiffness in one case, chondrodiastasis occurred instead of arthrodiastasis in one case with resultant lengthening of the femoral neck

Conclusions: Arthrodiastasis without soft tissue release is a valid option of treatment in cases with Perthes disease who would normally expect to have poor prognosis with conventional treatment.

Significance: Treatment of selected patients with perthes disease [high risk patients] in the early phase with arthrodiastasis alone give promising results.

\section{2}

\section{PONSETI VS. FRENCH PHYSIOTHERAPY FOR CLUBFEET:} WHICH IS BETTER?

B. S. Richards, C. E. Johnston, D. J. Sucato, J. A. Herring, J. G. Birch, K. E. Rathjen, L. A. Karol, S. Faulks, S. Signall

Purpose: The Ponseti and French physiotherapy methods for clubfoot correction have been successful in reducing the need for surgical releases. The purpose of this prospective study was to compare results of these two methods at one institution in patients having a minimum 24 months follow-up.

Methods: Beginning in 2001, patients under 3 months of age with previously untreated idiopathic clubfeet were enrolled. Parents selected the method of treatment. Ponseti's and Dimeglio's treatment protocols were strictly adhered to. Before treatment, each foot was rated using Dimeglio's numeric scoring system and grouping (moderate, severe, very severe). Outcomes were classified as good (plantigrade foot \pm TAL), fair (limited posterior release or tibialis ant transfer), or poor (complete PMR).

Results: Follow-up averaged 48.2 months (range 24-73 mos). The average pre-treatment Dimeglio rating of feet was similar between Ponseti (12.9) and French (12.7) [p = NS]. Overall, Ponsetitreated feet tended to fare better but this was not statistically significant. The difference between the two groups was significant (in favor of Ponseti) in the "severe" category, which comprised the majority of feet in each treatment method [p $<0.03]$.

\begin{tabular}{llll}
\hline Overall outcomes & Good & Fair & Poor \\
\hline Ponseti (209 feet) & 151 feet $(72 \%)$ & 26 feet $(13 \%)$ & 32 feet $(15 \%)$ \\
PT (100 feet) & 63 feet $(63 \%)$ & 21 feet $(21 \%)$ & 16 feet $(16 \%)$ \\
\hline
\end{tabular}

Conclusions: At nearly 4 years follow-up, both methods significantly reduce the need for PMR. Overall, the outcomes for both groups were similar but in the "severe" category, the Ponsetitreated feet, which routinely receive a heel-cord tenotomy, achieved significantly better outcomes than the physiotherapy method, which here-to-fore did not include routine heel-cord tenotomy.

Significance: If physiotherapy is utilized, more frequent use of percutaneous heel-cord tenotomy may decrease the need for future posterior releases.

\section{3}

\section{THE ULTRASONOGRAPHIC EVALUATION OF THE PROCESSES OF RECONSTRUCTION-REGENERATION OF AN ACHILLES TENDON AFTER BEING TREATED BY PONSETI'S METHOD FOR CONGENITAL TALIPES EQUINOVARUS}

\section{A. Sionek, C. Jarosaw, G. Bartomiej, S. Chaberek, S. Pietrzak}

Purpose: The aim of this study was a comparative ultrasonographic evaluation of the dynamics of reconstruction-regeneration of Achilles tendons after treatment of idiopathic clubfoot at infants by means of Ponseti's technique.

Methods: The prospective examination was applied to 25 patients $(6$ girls and 19 boys) with unilateral clubfeet, at who after serial casting with the minimum 6 casts placed, a percutaneus tenotomy of the 
Achilles tendon was performed. The age of the patients at the moment of the intervention was between 6 and 10 weeks (average 8 weeks). The control group consists of Achilles tendons in feet which were not deformed. The ultrasonographic examination was done in prone position with the use of linear probe of the $10 \mathrm{MHz}$ frequency. The head of the transducer was put along the long axis of the Achilles tendon in its distal part, obtaining a longitudinal cross section. The first ultrasonographic examination was done on the day proceeding the tenotomy, the subsequent examinations were done in the $6,12,24$ and 60 weeks after the tenotomy. There was registered a digital picture of the sonograms with the marked examined area corresponding to a distal part of the Achilles tendon. The examined area was defined mathematically by using a parameter called fractal dimension which is a measure of self-alikeness and order of the examined structure.

Results: It was noticed an increase in value of the fractal dimension in the subsequent examinations, being a proof of the progressive processes of regeneration-reconstruction in Achilles' tendons. The difference was statistically significant $(P<0.05$; Student's $t$ test). The most intensive process of reconstruction was noted between 6 and 12 week dating from the operation performed. In the following examinations ( 24 and 60 week) was observed gradual decrease in the growth of the value of marked fractal dimension which might indicate a decrease in the dynamics of reconstruction of the area of the examined Achilles tendon.

Conclusions: By means of quantity method of the analysis of the sonographic picture, it was demonstrated that after 60 weeks the structure of the treated Achilles tendon is not fully reconstructed. Significance: Although the results of Achillles tendon reconstruction-regeneration are widely documented ultrasonographically and histologically, we did not find the studies using fractal dimension for evaluating this process.

\section{4}

\section{COMPLICATIONS OF THE DISPLACED FRACTURES OF THE RADIAL NECK IN CHILDREN: 96 CASES TREATED WITH ELASTIC INTRAMEDULLARY NAILING}

\section{P. Journeau, J. Métaizeau, N. Moh-Ello, P. Lascombes, T. Haumont}

Purpose: Fractures of the radial neck in children have a good prognosis in non- and moderate displacements. Nevertheless, the severe displacements lead to complications, mainly after open reduction. The aim of this study is to present the complications of the displaced fractures treated by the Metaizeau's technique, described in 1981.

Methods: This retrospective study is related to 96 fractures in 95 patients treated by elastic intramedullary nailing (ESIN) between 1979 and 2004. 77 fractures were treated exclusively by ESIN, 13 were treated by ESIN associated with percutaneous pinning, and 6 were treated by open reduction and ESIN. According to Metaizeau's classification, 9 grade II, 47 grade III and 40 grade IV (over $80^{\circ}$ tilt). Mean age was 10 years $(4-15) .36$ patients presented 45 associated injuries of the elbow including fracture of the olecranon (24), ulna (8), elbow luxation (7), fracture of forearm (3), medial epicondyle (2) and coronoid (1). Results were evaluated with functional (range of motion, pain) and radiological (residual tilt at FU) criteria, as well as study of the complications.

Results: The mean follow-up is 14.5 months ( 2 months to 5 years). At FU, the functional results were excellent $(82.2 \%)$, good $(11.1 \%)$, fair $(2.2 \%)$ and bad $(4.4 \%)$. 6 cases were lost for FU. The radiological results were excellent $(65.5 \%)$, good
$(17.7 \%)$, fair $(2.2 \%)$ and poor $(14.4 \%) .13$ bad results were related to one residual tilt over $40^{\circ}, 10$ fractures of the radial neck associated with other fractures including 3 open reductions, and 2 isolated fractures with an open reduction. The complications were 5 necroses, 3 non-unions, 3 peri articular ossifications, 2 epiphysiodeses, 1 hypertrophy of the radial head and 1 tilt $40^{\circ}$.

Conclusions: The principal factors of bad results in our survey were the initially associated osseous injuries and especially the open reduction. The 6 cases with an open reduction were associated with major complications. However, when reduction was possible with a $\mathrm{K}$-wire, the quality of the results was greatly improved. The main advantage of ESIN is the possibility to obtain an excellent reduction and good stability of the fracture. This method is recommended in conjunction with $\mathrm{K}$ wire reduction in severely displaced fractures.

Significance: Complications of fractures of the radial neck in children are related to the associated injuries of the elbow and to surgical open reduction which must be avoided as often as possible. The sequelae were dominated by radial head necrosis and non union. Use of the K-wire combined with the ESIN decreases the severity of the complications.

\section{5}

\section{INTERMEDIATE TO LONG TERM RESULTS OF A MINIMALLY INVASIVE TECHNIQUE FOR THE TREATMENT OF UNICAMERAL BONE CYSTS}

\section{J. Dormans, A. Arkader, A. Manteghi, G. Mik}

Purpose: Unicameral bone cysts (UBCs) are benign bone lesions commonly encountered in pediatric patients. Several treatment methods have been described (e.g., observation, steroid injection, bone marrow injection, curettage and bone grafting) with variable results and high recurrence rate. We previously reported the shortterm results of a minimally invasive technique that include the novel combination of intra-medullary decompression and percutaneous grafting with medical-grade calcium sulfate pellets (MGCS), with excellent outcome and low recurrence rate. The purpose of this study was to review the intermediate to long-term results.

Methods: The technique consists of aspiration and cystogram, curettage and removal of cyst lining, frozen section is perform whenever the diagnosis is uncertain, intramedullary decompression, and grafting with MGCS. This was a retrospective evaluation of this technique with a minimum follow-up of 24 months. Complete response (CR) was defined as cortical thickening and opacification of greater than $95 \%$ of the cyst; partial response (PR) as cortical thickening and opacification greater than $80 \%$; incomplete healing $(\mathrm{IH})$ as opacification of less than $80 \%$ with no cortical thickening.

Results: We identified 55 patients with an average age of 10.8 years (range 1.3-18). There were 36 males. 41/55 lesions occurred in the humerus and femur. The average follow-up was 37 months (range 24-70), 35 patients were clinically and radiographically followed for an average of 36 months (range 24-70), while 25 patients were clinically followed for an average of 47 months (range 24-84). 44/55 (80\%) patients had a CR or PR following initial surgery; 7 obtained a CR or PR after a repeat surgery (cumulative healing rate (CHR) of $94 \%$ ); 2 needed a 3 rd surgery (CHR 98\%); 1 needed a 3rd repeat surgery (CHR 100\%). There were no major complications associated with the procedure. Two patients had a superficial infection that resolved with oral antibiotics. There were no adverse reactions to the MGCS. 
Conclusions: This study provides an intermediate and long-term outcome of a minimally invasive technique for the treatment of UBCs. Although some patients required a repeat procedure, we were able to obtain $100 \%$ of complete or partial response at minimum 24 months follow up.

Significance: UBCs are very common benign lesions in children. Although several treatments have been described, the reported recurrence rate is high. We obtained excellent long-term results with low recurrence and complication rates with a minimally invasive technique.

\section{6}

\section{LONG-TERM PROGNOSIS OF EXTREMITY LOCATED EOSINOPHILIC GRANULOMAS IN CHILDREN MANAGED ONLY BY AN OPEN BIOPSY}

\section{B. Erol, B. Kocaoglu, H. A. Akgulle, O. Ofluoglu}

Purpose: Various treatment methods including curettage, intralesional steroid injections and radiotherapy have been used for local control of eosinophilic granuloma. In this prospective study, we evaluated the long-term prognosis of eosinophilic granulomas located in the extremity bones of children that were managed only by an open biopsy procedure.

Methods: Between 2000 and 2005, in two different hospitals by two seperate surgeons, an open biopsy was applied to 13 children (seven girls/six boys, mean age 5; range 2-9) with a preoperative diagnosis of eosinophilic granuloma. The differential diagnosis primarily included Ewing's sarcoma and osteomyelitis, but some other specific benign and malignant bone tumors as well. The location of the lesions was pelvis (4), tibia (3), proximal femur (2), femoral diaphysis (2), clavicula (1) and ulna (1). Pain, local tenderness, mild swelling, and limping were the main clinical findings. Systemic findings including fever, elevated ESR and leukocytosis were present in five patients. Besides the evaluation of primary lesion by radiographs and MRI, involvement of other skeletal sites (by bone scan or radiographs) and internal organs (gastrointestinal and respiratory systems, skin) were also searched. An open incisional biopsy with a frozen section was applied to all lesions. All children had regular clinical and radiographic followup (by radiographs and BT when required) postoperatively; the average follow-up was 34 months (range 27-83 months).

Results: None of the cases had involvement of other skeletal sites or internal organs. All biopsies were consistent with the diagnosis of "eosinophilic granuloma". None of the children required an additional surgical procedure after the biopsy. The patients with pelvis and lower extremity involvement were completely asymptomatic and able to bear full-weight at 6 weeks after the operations. Full range of motion of the upper extremities was possible in fourth postoperative week following clavicula and ulna biopsies. Gradual radiographic reconstruction of the destructive areas was completed at the end of 1 year for all lesions. None of the patients experienced recurrence and at the final follow-up they all had complete clinical and radiographic healing.

Conclusions: Eosinophilic granuloma has a high potential for spontaneous healing. Our study showed that an open biopsy to confirm the diagnosis is the only required procedure for the management of eosinophilic granulomas located in the extremities of children.

Significance: Open biopsy to confirm the diagnosis is the only required procedure for the management of eosinophilic granulomas located in the extremities of children.

\section{THE LONG TERM FOLLOW-UP RESULTS OF RECONSTRUCTION OF DEFECTS FOLLOWING BONE TUMOR RESECTIONS BY DISTRACTION OSTEOGENESIS}

\section{Yildiz, I. Cicek, M. Basbozkurt, M. Kurklu, Y. Yurttas}

Purpose: Survival after resection of musculoskeletal tumors continues to increase, thereby making limb salvage an important alternative for the restoration of functions. Reconstruction of bone defects following en bloc resection of malign or aggressive benign bone tumors is one of the major problems in orthopedic surgery. The aim of this study is to report our long-term follow-up experience in the reconstruction of bony defects following en bloc resection of bone tumors by Ilizarov's distraction osteogenesis using bone transport method on which there was limited report in the literature.

Methods: We performed en bloc resections in 9 patients with bone tumors from October 1991 to January 2000. Two patients were female and 7 were male. The average age of the patients was 11.3 (7-15). Surgical staging, which determines treatment, was done according to diagnostic algorithm. Histological diagnosis was osteosarcoma in 4 cases, Ewing's sarcoma in 2, giant cell tumor (aggressive) in one, osteofibrous dysplasia (latent) in one and osteoblastoma (aggressive) in one. Uniplanar external fixator was applied to one case and circular external fixator to eight cases. The average followed up period was 94.1 months (62-164). The function of the affected limb was assessed according to MSTS functional scoring system in the follow-up periods.

Results: We observed three pathologic fractures and these were treated with AO plate osteosynthesis. One patient was dead because of brain metastasis. The functions were excellent in five patients, good in four according to MSTS functional scoring system.

Conclusions: Ilizarov bone transport technique is an alternative method for the restoration of bone defects formed following en bloc resection in the treatment of bone tumors. Despite the high disabling complication rates seen in the patients who were reconstructed with the other methods, tumoral defect closure by distraction osteogenesis has limited complication rate in the long time follow-up period.

Significance: There were limited reports in the literatures about the long-term follow-up experiences in the reconstruction of bony defects following en bloc resection of bone tumors by Ilizarov's distraction osteogenesis using bone transport method.

\section{E-Poster Presentations}

\section{8}

\section{DEGA PERICAPSULAR ACETABULOPLASTY FOR THE TREATMENT OF SUBLUXATED AND DISLOCATED NEUROLOGIC HIPS: LONG TERM FOLLOW UP}

A. Khairouni, A. Presedo, B. Ilharreborde, E. Morel, F. Fitoussi, G. F. Penneçot, K. Mazda, P. Souchet

Purpose: The aim of this study was to determine if the relocated hips remained stable and well-contained at skeletal maturity, after Dega acetabuloplasty. 
Methods: Sixty-five patients underwent Dega pericapsular acetabuloplasty between 1994 and 2006, at a mean age of 8 years. Fifteen patients that had reached skeletal maturity at latest follow up were included in the study. The operation was indicated for dislocated or subluxated hips with increasing Reimers percentage observed on subsequent radiographs. Anteroposterior pelvis radiographs were evaluated preoperatively, postoperatively and at final follow-up. We measured the acetabular index (AI), the center-edge angle (CEA), the Reimers percentage (RP) and the neck-shaft angle (NSA).

Results: All patients with cerebral palsy but one underwent bilateral femoral shortening varus-derotation osteotomy and Dega pericapsular acetabuloplasty. One patient with poliomyelitis and another patient with myelomeningocele had isolated acetabuloplasty. Mean follow up was 8 years. Mean preoperative Reimers percentage was $54 \%$, mean acetabular index was $29^{\circ}$, mean center-edge angle was $-9^{\circ}$ and mean neck-shaft angle was $159^{\circ}$. At final follow up the mean value of these parameters were: Reimers percentage $16 \%$, acetabular index $13^{\circ}$, center-edge angle $24^{\circ}$ and neck-shaft angle $129^{\circ}$. At final follow up, 20 hips remained stable and painless. No cases of avascular necrosis were observed. Two patients recovered ambulation.

Conclusions: Dega pericapsular acetabuloplasy allows correction of posterolateral acetabular deficiency in neurologic hips. The restoration of a correct acetabular shape prevents reccurrence of subluxation despite increase of the femoral neck-shaft angle. In our experience, femoral shortening avoids open reduction and prevents avascular necrosis of the femoral head.

\section{9}

\section{TRANSFER OF PECTORALIS MAJOR MUSCLE IN ARTHROGRYPOSIS-RESULTS AND FACTORS INFLUENCING THE OUTCOME 8-11 YEARS AFTER SURGERY}

\section{J. Chomiak, P. Dungl}

Purpose: Transfer of 3 distal parts of pectoralis major muscle was used to restore active elbow flexion in patient with arthrogryposis. The purpose of this study was to evaluate the results $8-11$ years after the surgery and factors influencing the results.

Methods: From 1996 to 1999, elbow flexion was reconstructed in 9 upper extremities by 5 patient aged 4.3-9 years. The patients were clinically evaluated according to the same subjective and objective assessment and examined electromyographically before the surgery and during the follow up. Last clinical examination was provided 8-11 years after the surgery in patients aged $14-18$ years. Results: 3-4 years after pectoralis major transfer, 6 very good and good results were achieved, because the average active ROM of elbow ranged from 15 to $95^{\circ}$. The power of elbow flexion was graded as 4 and $4+$. This method was unsuccessful in the remaining 3 cases, when the patients were not able to reach the mouth with the hands because of limited elbow flexion. 8-11 years after the surgery, the results were similar to the short-term results. 5 extremities remain very good and good, one deterioration was obvious. The ROM of elbow changed, namely the extension was mostly reduced even in very good and good evaluations (the average decrease was $13.8^{\circ}$, the range -20 to $+10^{\circ}$ ). The active flexion was not changed in 5 elbows, or it was increased (the average increase $4,4^{\circ}$, the range $0-15^{\circ}$ ). The final limitation of extension $\left(30-50^{\circ}\right)$ does not restrict using the hands for perineal hygiene and the final active flexion $\left(85-100^{\circ}\right)$ allows elevating the hands to the head for feeding and toilet.
Conclusions: From the analysis of unsuccessful results 3-4 years after the surgery it was obvious that all cases were related to very limited preoperative passive flexion of the elbow; restricted movement of the shoulder and failed distal fixation of the transferred muscle. After 8-11 years, the active elbow flexion and extension for raising the hands to the mouth and for toilet needs, respectively, remain in majority previously successful cases. Because most children reached the skeletal maturity, no further shortening of the transferred muscle and limitation of extension is expected. These findings do not concur with the literature reports. According to our results, the transfer of the pectoralis major represents the efficient method for permanent restoring of bilateral active elbow flexion with the remaining functional extension. The ROM does not change significantly after having reached the plateau 2 years postoperatively.

Significance: The prerequisites for successful results are a minimum of $90^{\circ}$ of passive flexion of the elbow before the surgery, the active shoulder abduction of $80-90^{\circ}$, long term rehabilitation and successful fixation of the transferred muscle to the forearm.

\section{0}

\section{DO ANKLE FOOT ORTHOSES IMPROVE BALANCE IN CHILDREN WITH SPASTIC DIPLEGIA?}

\author{
D. Jackson, D. M. Eastwood, E. Main, M. Mayston
}

Purpose: In neuromuscular conditions, ankle foot orthoses (AFO) are thought to prevent deformity and improve functional balance by increasing the base of support, stabilizing the ankle joint and influencing the kinematics of more proximal joints. This study was designed to evaluate the role of fixed AFOs on balance in children with spastic diplegia.

Methods: Twelve children (age 7-15 years) with spastic diplegia were recruited. All were community ambulators (GMFCS II/III). All had used AFOs for 12 months. Each child was asked to walk four times along the GAITRite electronic walkway at their preferred speed: barefoot, in shoes alone and in AFOs with shoes. The order of the walks was randomized limiting the effects of fatigue and confidence. Normalized velocity, cadence, stride length and percentage of gait cycle in single leg support were selected as surrogate measures of stability. The child's balance during other functional activities was assessed with the paediatric balance scale (PBS). All data was collected on the same day by the same therapist. A two-way analysis of variance (ANOVA) was conducted to explore any differences in gait between the various walking conditions and post hoc comparisons tested where these differences lay. Friedman's test tested for differences in PBS scores between subjects and between conditions.

Results: Significant improvements were seen in mean values for normalized velocity $(P=0.02)$, stride length $(P<0.01)$ and percentage of gait cycle in single leg support $(P<0.01)$ in footwear with AFOs compared to barefoot. Whilst there were also improvements in mean values for these parameters in shoes alone compared to barefoot, only the difference in stride length reached statistical significance $(P<0.01)$. There were no significant differences in PBS scores in shoes alone or in combination with AFOs compared with walking barefoot.

Conclusions: AFOs improved balance during gait but, in children with spastic diplegia, had no significant effect on balance during other activities as reflected by the PBS. Whilst the shoes themselves had a positive impact on the child's gait, the most significant effects were seen in the AFO-footwear combination. 
Significance: A wide variation in the child's responses to shoes and AFOs emphasizes the need for the ongoing evaluation of orthotic use. Current advice regarding the use of AFOs and the choice of footwear should consider the benefits they offer in gait as well as prevention of deformity.

\section{1}

\section{FIFTEEN YEARS EXPERIENCE WITH DEGA ACETABULAR OSTEOTOMY IN CHILDREN WITH CP}

\section{A. Borowski, A. Grzegorzewski, B. Pruszczyński, M. Synder}

Purpose: Untreated hip joint in cerebral palsy children leads to subluxation or dislocation caused by muscle imbalance often with associated painful arthritis. The forces gradually changing the shape of the acetabulum, which becomes more elliptical, more disposed for femoral head dislocation. To improve coverage of the femoral head (posteriorly and laterally) by the acetabulum, acetabular pelvic Dega osteotomy was performed in children with spastic hip disease. The aim of this study is to assess the long-term results of this procedure.

Methods: Between 1992 and 2000, 136 cerebral palsy children (diplegia/quadriplegia) were treated by multilevel soft tissue surgery. Among them 95 children (49 boys and 46 girls) required surgical interventions for hip problems. In 18 cases, (mean age 11.2 years) with limitation of adduction of $20^{\circ}$ and migration index (MI) above $60 \%$ (average $87 \%$ ), femoral varus osteotomy combine with Dega pelvic osteotomy was performed. The study was based on clinical examination, parents' satisfaction questionnaire and radiological findings. Mean follow up was 10 years. Results: The functional improvement was observed in children with bone surgical procedure. Significant correction of the position of femoral head into acetabulum allowed for sufficient abduction of the leg with mean increase of $18^{\circ}(P<0.05)$. In ambulatory patients, gait pattern had change to less energy pattern. In non-ambulatory patients, ability of perineal care improved. In all children decrease in hip pain was observed. The unsatisfactory results were seen in 2 children, among non ambulatory patient, 6 years after surgery-wind blown deformity with recurrent dislocation. No bone fracture and avascular necrosis of the femoral head had been noticed.

Conclusions: With a properly planned approach, bone surgery can bring good functional results. Dega's acetabular osteotomy allows for an excellent correction of head coverage in superior, lateral and posterior deficiency of the acetabulum.

Significance: Dega acetabular osteotomy is one the best adequate surgical procedure to improve coverage of the femoral head in spastic hip.

\section{2}

\section{ADDUCTOR MYOTOMY VERSUS TRANSFER TO THE ISCHIADIC TUBER IN THE PROPHYLAXIS HIP DISLOCATION. IS IT WORTH TO EXPAND THE SURGERY?}

\section{A. Borowski, A. Grzegorzewski, E. Pogonowicz, M. Synder}

Purpose: Spastisity in cerebral palsy leads to limitations of range of motion (ROM) and gradual contractures of the joints. The muscle imbalance results in subluxations or dislocations of the hip. There are various methods of soft-tissue surgery offered to prevent the hip dislocation. The aim of the study is to compare clinical (ROM) and radiological (migration index-MI) results of the myotomy of the hip adductor muscles versus the adductor muscles transfer to the ischiadic tuber in the treatment of the subluxation and prophylaxis of the dislocation of spastic hip.

Methods: The study includes 36 ambulatory children (69 hips) with spastic diplegic pattern involvement who had underwent surgery between 1987 and 2002. In 21 patients (41 hips) myotomy of the adductor longus, adductor brevis and gracilis without neurectomy of the anterior branch of the obturator nerve were performed (group A). The transposition of the adductor muscles tendons (adductor longus, adductor brevis and gracilis) to ischiadic tuber was done in 15 patients, 28 hips (group B). The average age at the time of surgery in group A was 10 years, ranged from 3 to 17 . In the group B, the average age was 8 years, ranged from 3 to 16 . The study consists on clinical examination, gait pattern and radiological findings. Hospital stay and time to recovery after surgery were compared as well as manner and time of immobilization. The average follow up in the group A was 5.6 years (5-8), in the group B the average follow up was 11.9 years (7-20).

Results: There was no difference in ROM in both groups before surgery. As well as at the last follow up. ROM improved significantly in both groups; however, equally decreased with time. There was no difference in radiological results between these two groups before and at the final follow up. There was significant difference in hospital stay time and recovery time between both groups (group A, average hospital stay was 6 days, recovery 3 months, group B, average hospital stay was 9 days, recovery 4 months). There was no hip joint immobilization in the group A, in the group B a cast was used to forced abduction after surgery for 6 weeks.

Conclusions: Concerning the results above, we found out that the adductor myotomy was superior because of easier way of management, shorter time of hospital stay, shorter time of recovery and the hip joint immobilization was not necessary.

Significance: Adductor myotomy in the prophylaxis of spastic hip joint subluxations is superior to adductor transfer.

\section{3}

\section{CEREBRAL PALSY RESULTS IN HIGHER EXPRESSION OF THE FAST BUT FATIGABLE MYOSIN HEAVY CHAIN IIX ISOFORM IN BOTH WRIST FLEXORS AND WRIST EXTENSORS IN COMPARISON WITH HEALTHY CHILDREN}

\section{S. Gantelius, E. Pontén, Y. Hedström}

Purpose: Children with spastic cerebral palsy (CP) often show a typical pattern in the upper extremity with a flexion contracture of the wrist even though the stretch reflex often is increased in both flexors and extensors. The myosin heavy chain (MyHC) isoforms give the muscle fiber different characteristics such as fast or slow contraction velocities and different endurance. For example, the MyHC IIx isoform contracts fast but fatigue easily. The purpose of this study was to examine if there is a different expression of the MyHC isoforms in wrist flexors compared to wrist extensors in children with $\mathrm{CP}$ in comparison with healthy children.

Methods: Muscle biopsies were taken from wrist flexors (FCU, FCR) and wrist extensors (ECRB, ECU) during tendon transfer surgery in children with $\mathrm{CP}(n=9)$. Control biopsies were taken 
from the same muscles in healthy children $(n=7)$ during open reduction of forearm fractures. The expression of the MyHC I, IIa and IIx isoforms were determined on silver-stained $6 \%$ SDSPAGE.

Results: Significantly higher expression of the MyHC IIx isoform was seen in wrist flexors than in wrist extensors in both healthy children ( 16 vs. $3 \%, p=0.01)$ and children with CP (40 vs. $20 \%$, $\mathrm{p}=0.01)$. Children with $\mathrm{CP}$ had a significantly higher expression of the MyHC IIx isoform in both wrist flexors (40 vs. $16 \%$, p 0.001 ) and wrist extensors ( 20 vs. $3 \%, p=0.05$ ) in comparison with healthy children.

Conclusions: The higher expression of the fast MyHC IIx isoform in flexors than extensors of the wrist probably reflects a different use of the muscles during grasp and grip, although both are wrist stabilizers. Decreased use of the hand could in CP account for the increased occurrence of the MyHC IIx isoform in the spastic muscles.

Significance: Treatment of flexion deformities in children with CP is one of the challenges in pediatric orthopedic surgery. Knowledge about the contractile properties in spastic muscle could open up to new treatment protocols. Training might decrease the expression of MyHC IIx isoform.

\section{4}

\section{PERCUTANEUS SAN DIEGO OSTEOTOMY}

\section{G. de Coulon, A. Kaelin, D. Ceroni, V. de Rosa}

Purpose: Subluxated and luxated hip in cerebral palsy patients is frequently treated by San Diego osteotomy. Those patients consistently have anterior and/or posterior and/or lateral insufficiency of the acetabulum. The surgical procedure can provide good redirection of the acetabulum. This surgery is usually performed with a modified Smith Petersen approach. We would like to describe the technique and present our results done with this new approach.

Methods: The patient is in decubitus dorsalis, we first carry out the varus and derotation osteotomy of the proximal femur. Subsequently - with the image intensifier and a Kirschner wire-we project the right place for the acetabulum osteotomy and we draw the first line on the skin. A 2nd line will start off at the greater trochanter and will proceed equidistant between the posterior and anterior iliac spine in proximity. The place for the incision is determined by the point at which these two lines cross each other: it will be between 2 and $3 \mathrm{~cm}$ long. Making use of a Cobb, we go through the fascia lata and the gluteus up to the external iliac cortex. We scrape the muscle from the sciatic notch to the antero-inferior iliac spine to free the area were the osteotomy will be performed. This procedure is supervised with the image intensifier with alar and obturatric views. Osteotomy sets off with a $1 \mathrm{~cm}$ chisel following the San Diego procedure. The bone previously taken during the femur osteotomy is now wedged and crossed with a $2 \mathrm{~mm}$ Kirschner wire. Subsequently — with a $1.5 \mathrm{~cm}$ wide chisel - we bend the acetabulum roof and push the graft inside the osteotomy with a trocar to accomplish the right position.

Results: Eighteen patients have been treated with this technique revealing a Reimers index of $68 \%$ pre op to $6 \%$ post op and an acetabulum index of $32^{\circ}$ pre op to $14^{\circ}$ post op. All these patients have been followed until bone consolidation. Neither during the procedure nor in the follow-up has no complication been observed. Conclusions: This approach is more straightforward and implies less muscle scraping.
105

\section{KINEMATIC DEVIATIONS OF THE INVOLVED \& THE SOUND LIMB IN HEMIPLEGIC INDIVIDUALS WITH CEREBRAL PALSY}

D.G. Pasparakis, C.M. Nestoridis, M.E. Pentarakis, M.N. Tziomaki, N.G. Darras, N.M. Papandreou

Purpose: The further investigation of movement disturbances of both the hemiplegic and the unaffected limb in hemiplegic CP subjects based on Gait Analysis graphs.

Methods: The kinematic details of 17 hemiplegic subjects were analyzed from the ELEPAP Gait Analysis Center database. The mean age was $12.1 \pm 4.6$ (7 Male and 10 Female, 10 rights and 7 left). The kinematic analysis was performed using BIOKIN motion analysis system. A deviation matrix was created in order to locate graphs of the lower limb joints on the sagittal plane that are not following the Normal waveform.

Results: In $82 \%$ of the subjects, an asymmetrical movement of the pelvis in the transverse plane was documented with the hemiplegic side to follow the uninvolved side. In $12 \%$ of the subjects, the pelvis movement was normal, while $6 \%$ presented the hemiplegic side to precede the unaffected side. No statistically significant difference was found between the step length proportions in one stride (Hemiplegic step length $48 \% \pm 3.3$ - Uninvolved side step length $52 \% \pm 5.7$ ). However, a significantly shorter duration of stance phase on the hemiplegic side was found (Hemiplegic limb $60.8 \% \pm 3.5$, Uninvolved limb $64.4 \% \pm 3.1 \mathrm{p}=0.001)$. The graph deviation matrix on the sagittal plane revealed the following frequency of abnormalities for the lower limb joints:

\begin{tabular}{llll}
\hline Hemiplegic limb & Hip 64.7\% & Knee 58,8\% & Ankle 74.5\% \\
Uninvolved limb & Hip 70.6\% & Knee 47.1\% & Ankle 35.3\%
\end{tabular}

Conclusions: Deviations of both lower limb joints normal motion were located. The hemiplegic subjects move asymmetrically with the affected side to follow the uninvolved side. On the hemiplegic side the duration of support is shorter and the ankle movement in the sagittal plane is more involved than the hip and the knee. The uninvolved limb compensations are more proximal situated, influencing mostly the hip and in a lesser degree the knee and the ankle. Significance: Various types of hemiplegic kinematic behavior, based on Gait Analysis graphs, have been identified; however, movement disturbances of both the hemiplegic and the unaffected side, are still rather lacking. Treatment interventions to improve the walking quality of hemiplegic cp individuals should focus not only on the hemiparetic limb but on the functional limitations of the sound limb as well.

\section{6}

\section{ARE RECURRENT DISLOCATION AND AVN FOLLOWING PROXIMAL FEMORAL VARUS OSTEOTOMY IN CHILDREN WITH CEREBRAL PALSY PREVENTABLE?}

\section{Ghanem, F. Dagher, K. Kharrat, R. Khalifeh, S. El Hage}

Purpose: The purpose of this study was to report mid-term results of femoral varus osteotomy FVO in children with cerebral palsy, 
and to identify risk factors for complications, particularly avascular necrosis AVN and recurrent dislocation RD.

Methods: A retrospective review was conducted on 50 cerebral palsy children, who underwent FVO, with or without derotation or open reduction, in 89 hips, at an average age of 7.4 years. The pattern of cerebral palsy, the severity of functional disability, age at surgery, type of surgery and radiographic measurements were recorded preoperatively, postoperatively, and at an average follow-up of 6 years (2-13 years), and correlated (when applicable) with the development of AVN, and/or RD.

Results: Sixty-five hips were treated surgically for subluxation, 12 for dislocation and 12 to establish symmetry. Among the hips treated for subluxation, 12 presented moderate residual subluxation postoperatively (Reimers index RI $42 \%$ ) which progressively stabilised (RI 22\%) at last follow-up. Average age at surgery in these patients was 5.6 years. Among the 53 well reduced hips postoperatively, a new subluxation developed in 12 , with a mean postoperative neck-shaft angle of $135^{\circ}$ (compared to $119^{\circ}$ for the remaining 41 hips of this group) and $145^{\circ}$ at last follow-up; These 12 new subluxations had an average preoperative acetabular slope of $32^{\circ}$ at surgery, but did not undergo pelvic osteotomy. Two of the 12 dislocated hips were subluxed at last follow-up. Those operated to establish symmetry were all stable. Avascular necrosis was observed in 33 hips (37\%): 26 minor and seven severe. The two statistically significant risk factors for AVN were a high preoperative RI, $63 \%$ for the AVN group v/s $44 \%$ for unaffected hips, $\mathrm{p}<0.05$ ), and older age at surgery, 8.7 years for the AVN group v/s 6.3 years for unaffected hips, $\mathrm{p}<0.05$ ).

Conclusions: The main risk factors for secondary dislocation following proximal femoral varus osteotomy despite respect of symmetry seem to be insufficient correction of pre-existing valgus and uncorrected acetabular dysplasia. Secondary improvement of residual subluxation may be the result of remodeling. The risk of AVN following proximal FVO is relatively high and depends on the severity of initial dislocation and old age at surgery.

Significance: Sufficient correction of pre-existing valgus and acetabular dysplasia is essential to prevent dislocation or subluxation recurrence. The risk of AVN following proximal femoral varus osteotomy is relatively high and depends on the severity of initial dislocation and old age at surgery.

\section{7}

\section{APPLICATION OF GAIT ANALYSIS FOR EVALUATION OF GAIT PATHOLOGY IN YOUNG CEREBRAL PALSY CHILDREN}

\section{Bonikowski, K. Sakawska, . Grabczewski, Z. Kalinowski}

Purpose: Widespread of BTX-A treatment during last years and orthosis development enables taking early interventions in cases of spastic cp. Gait analysis is an important tool enabling gait abnormality diagnosis even in small children and taking up proper treatment.

Methods: $30 \mathrm{cp}$ children were examined in our gait lab. 20 with spastic diplegia aged $22-36$ months and 10 with spastic hemiplegia aged 19-36 months. All patients with hemiplegia were able to walk independently. All but two patients with diplegia were able to walk with assisted by other person. No patients had limited passive range of motion (ROM). All of the children were covered with NDT or Vojta rehabilitation programms from the first months of life. Before the examination none of them used orthosis, neither were they treated pharmacologically (BTX-A). The examination was performed with the Sybar 2.0 (Noldus
Holland) gait analysis system using surface EMG-Porti 16 (TMS Holland). Aspects assessed were kinematics of gait, angles were measured by the build in goniometer witch measures the angle formed by body segments in the 2-dimension space of the monitor window. Recording from the surface EMG were recorded from the following muscles: gastrocnemius medialis; tibialis anterior; semitendinosus, vastus lateralis.

Results: In cases of all patients with hemiplegia pathologic gait pattern was stated, type 2 according to Winters or type 3 according to Becher-knee hyper extension in midstance with heelrise, with true equinus, an absence of 1 st and restriction of 2 nd rocker and an excessive plantarflexion/knee extension couple with abnormal activation of the triceps surae muscle and prolonged activation of the vastus lateralis muscle. In cases of patients with diplegia one observed gait pattern Type 3 according to Becher: Knee extension in midstance with heelrise or gait pattern Type 4: knee-flexion in midstance with heel-rise (Becher) - a gait pattern characterized by hip and knee flexion in mid stance with abnormal activity of the Gastrocnemius, Hamstrings and Psoas muscles. It should be stressed that even in children aged 19 months secondary deformities like hyperextension of the knee joint during stance face were observed.

Conclusions: This study suggests that clinical gait analysis can be used successfully to diagnose gait pathology and qualify for early intervention in young children with $\mathrm{CP}$. We encountered no problems resulting from small children's anxiety or reluctance, preparation for the examination (placing the electrodes), as well as the examination itself, were treated as a play or game.

Significance: Gait disorders appear in cases of very young $\mathrm{cp}$ children, including secondary abnormalities, like knee hyper extension. Thus early treatment using proper methodology is very important.

\section{8}

\section{EPIDEMIOLOGY OF HOSPITALIZATION AMONG PATIENTS WITH CEREBRAL PALSY IN THE UNITED STATES FROM 1979 THROUGH 2003}

\section{H. Matsumoto, D. P. Roye, J. E. Hyman, M. G. Vitale}

Purpose: The purpose of this study is to examine trends in incidence and characteristics of hospitalization for Cerebral Palsy (CP) patients in the United States from 1979 through 2003.

Methods: The National Hospital Discharge Survey (NHDS) was utilized to investigate trends in hospitalization among $\mathrm{CP}$, characteristics of patients and procedures performed. By aggregating the weights on approximately 350,000 yearly discharges in both children and adults, roughly 40 million annual discharges were obtained.

Results: An incidence of hospitalization among CP patients decreased over the 24-year period from 4.2 cases to 2.5 cases per 100,000 . The average age of the patients increased from 14.0 years to 24.5 years. Male patients had higher incidence of hospitalization than female patients (mean annual relative risk $=1.3$ ). Blacks had higher rates of hospitalization than did Whites (mean annual relative risk $=2.0$ ). Quadriplegia was the most commonly reported form of $\mathrm{CP}$ in the last 15 years. Medicaid and private insurances were the two major pay sources; however, after 1995, Medicaid became the most common primary payer. The top five inpatient procedures performed in the average annual frequencies were orthopedic $(54.8 \%)$, neurogenic $(8.6 \%)$, radiological $(8.5 \%)$, gastrointestinal (GI, 7.8\%), and ear/nose/throat (ENT)-Dental (3.4\%). There was a downward trend in orthopedic 
inpatient procedures over the 24-year period. Common procedures through out the study period were tendon lengthening/transfer, hip osteotomy/adductor tenotomy, and other soft tissue procedures. There was an increasing trend in neurological inpatient procedures. In 1988, Dorsal rhizotomy first appeared on the database and became a common procedure along with other procedures such as application of intrathecal baclofen. There were decreasing trends in the average length of hospital stay and the deaths.

Conclusions: These data indicate a decreasing incidence of hospitalization which can be explained by expanded use of ambulatory treatments. An increasing trend in average age of hospitalized patients is presumably a result of the increase in CP survival rate. Over the 24 year period, orthopedic procedures remained the most common procedures performed. There seem to be, however, a downward trend in orthopedic inpatient procedures which is most likely due to increases in ambulatory surgeries. Further studies need to be done in order to explore the relationships between decreasing incidence of hospitalization and orthopedic procedures and increasing use of ambulatory treatments.

Significance: Although this study does not reflect the incidence of $\mathrm{CP}$ nor outpatient procedures performed on $\mathrm{CP}$ patient, these data adds important elements to establish health care policy and to allocate health care resources.

\section{9}

\section{TREATMENT OF UNICAMERAL BONE CYST BY SINGLE INJECTION OF ALPHA-BSM}

\section{E. Johnston, C. Tsai}

Purpose: To evaluate healing of unicameral bone cyst (UBC) following a single percutaneous injection of calcium triphosphate/ collagen $(\alpha$-BSM) reconstituted with saline, by using a new modified Neer classification and measurement of the lytic area radiographically.

Methods: 14 patients ages 6-19 treated $2002-2005$ by a single percutaneous lavage and $\alpha$-BSM injection were reviewed. Technique: following cystogram to outline the extent of the cyst, vigorous saline barbotage via two Jamshidi needles to "disrupt" the cyst wall was performed, followed by injection of $\alpha$-BSM "paste" until the cyst is filled. Outcome (mean f/u 25 mo., range 13-46) was graded by xray measurement of the change of area $\left(\mathrm{mm}^{2}\right)$ of residual cyst, width of the cyst wall cortex, plus clinical symptoms (e.g., pain, refracture) requiring further treatment.

Results: 9 UBC's were active, 5 latent. 13/14 lesions decreased in size, with mean area $=28.6 \%$ (range $0-104)$ at $\mathrm{f} / \mathrm{u}$ compared to pre-treatment area. Latent cysts resolved better, being only $7 \%$ (range 0-30) of pre-treatment size at $\mathrm{f} / \mathrm{u}$, compared to $40 \%(0$ 104) in active lesions. Larger cysts $>2,000 \mathrm{~mm}^{2}$ tended to decrease in area less than cysts $<2,000 \mathrm{~mm}^{2}$. Using the new classification, 5 cysts were grade 1 (complete healing), 4 grade 2 (residual lysis $<50 \%$ of bone diameter + thicker cyst cortex), 4 grade 3 (residual lysis $>50 \%$ bone diameter + thicker cortex), and 1 grade 4 (no effect, re-fracture $=$ failure). All patients except the one grade 4 refracture in a proximal femoral lesion were clinically asymptomatic.

Conclusions: 13/14 lesions were improved or healed (decreased cyst area, cortical thickening) with resolution of symptoms by this technique.

Significance: Multiple serial injections of steroid or marrow aspirate are no longer necessary to treat UBC.

\section{0}

\section{INTRAOPERATIVE RADIOTHERAPY IN BONE TUMORS IN CHILDREN: OUR EXPERIENCE}

A. J. Villa-Garcia, A. Garcia-Martin, A. M. Martinez-Serrano, J. L. Gonzalez-Lopez, O. G. Riquelme-Garcia

Purpose: Intraoperative radiotherapy is a type of external radiotherapy that combines, in sensible neoplasms, precision and high dose radiotherapy. Experience in bone tumors in children with this procedure is limited.

Methods: We report 6 children treated of musculoskeletal tumors (Ewing's Sarcoma, synovial sarcoma) since 1997, 5 boys and 1 girl with a median age of 14 years (range, 13-15 years). All patients were treated with chemotherapy, surgical excision and radiotherapy. After surgical excision of the tumor, with the operatory field exposed, all patients' received high dose radiotherapy focused in tumor area. The irradiation field included the tumor area plus an adequate wide margin and excluded the major vessels and nerves.

Results: The median follow-up was 43 months. 2 patients died. 1 patient presented pulmonary metastases. No significant wound complications were reported.

Conclusions: Intraoperative radiotherapy is an alternative to conventional external radiotherapy where available. It can be used in almost any tumor locations, allows ensuring surgical resection margins and protects external tissues from high-dose radiation.

Significance: High-dose Intraoperative radiotherapy combined with surgical excision and chemotherapy appeared to be a promising quality-of-life-oriented alternative to treating patients with sensible musculoskeletal tumors in chilhood.

\section{1}

\section{SURGICAL OUTCOME IN PATIENTS TREATED FOR HEMANGIOMA DURING INFANCY, CHILDHOOD AND ADOLESCENCE. A RETROSPECTIVE REVIEW OF 44 CONSECUTIVE PATIENTS}

\section{F. Canavese, B. C. Soo, J. I. Krajbich, S. K. Chia}

Purpose: Hemangiomas are the most common tumors in infancy and childhood, and account for $7 \%$ of benign soft tissue tumors. Diagnosis is usually made in infancy or childhood. There are only a few reports on the surgical treatment of these lesions, likely due to the fact that the lesions are quite vascular, have a tendency to infiltrate into the muscle and other tissues, and the recurrence rate is quite high.

Methods: We reviewed the outcome of surgical treatment on 44 consecutive children and adolescents with 47 surgically treated hemangiomas. Hemangiomas involving the face and the head were excluded.

Results: 11 lesions were surgically treated before the age of five, 20 lesions between the age of 5 and 12 years, 16 lesions were treated after the age of 12. Eleven lesions had intralesional resection, 33 lesions underwent marginal resection, 2 lesions were managed with wide resection and one lesion underwent radical resection. The overall recurrence rate was $22.2 \%$.

Conclusions: In the pediatric population it is desirable to perform definitive treatment whenever possible, thereby minimizing 
morbidity, both functional and psychological. Asymptomatic lesions should be monitored to confirm the diagnosis and to look for signs of progression. Symptomatic lesions can be treated with surgical excision if this does not result in significant functional impairment. A marginal resection may be used to treat most superficial soft-tissue tumors. Intramuscular hemangiomas pose a more difficult therapeutic problem. When the lesion is limited in size, it may be treated with wide local excision. However, if the lesion is more diffusely spread into the muscle unit, the morbidity created by extensive muscle resection has to be weighed against the morbidity of the condition or alternative treatment methods. Whenever possible a wide marginal resection should be carried out in order to minimize the risk of recurrence and avoid further surgical procedures. We recommend prolonged follow-up of these patients, as $7 \%$ of the patients included in this study required follow-up for more than 10 years due to multiple recurrences of these lesions.

\section{2}

\section{OUR EXPERIENCE IN TREATMENT OF BENIGN LYTIC BONE LESIONS IN THE LIMBS BY THERMOABLATION IN CHILDREN}

\section{F. M. Lotito, A. De Caro, V. Iaccarino}

Purpose: We would like to emphasize the efficacy, through a percutaneus approach, of the thermoablation in lytic bone benign lesions in children. The excellent results obtained in Osteoid Osteoma (O.O.) allow us to perform the same treatment even in other benign lytic bone lesions. We point out the necessary attentions in order to obtain excellent results using this miniinvasion technique.

Methods: From 2003 and 2006 we treated 15 children affected by osteolytic lesion of the limbs. O.O. was diagnosed through clinical and imaging assessment in 13 cases. Response at acetylsalicylic drugs and typical aspects of the "nidus" at the TC_Scan, gave us high confidence to diagnose an O. O. while in two cases, despite the fact that the lesions were not typical, their radiological and clinical aspect appeared a benign lesion. 12 male and 3 female with a mean age of 11 years old (min.3-max.18) were our cohort. The lesion site was in 5 cases the upper femur, in 2 cases femur shaft, in 3 cases the tibia, 3 cases the body of the talus and 2 cases the humerus. Delay time to rich a diagnosis, from the initial symptoms, was a mean of 11 months (min 6 months-max 24 months). In one child, a second lesion in a tibia appeared after a previous surgical ablation in a femur 3 years before. In another case it was a recurrence. All the patients were treated in general anesthesia, under TC-Scan guidance. We did not perform any histological assessment if the lesion had typical aspects of the O.O., while in the two not-typical lesions; we drew a sample for a histological diagnosis, just before performing thermoablation. Chondrobalstoma was the further diagnosis. Thermoablation was done by a Radionics Equipment, using a special "cold tip" needle with a length between 7 and $10 \mathrm{~mm}$. The exposure time was $6 \mathrm{~m}$ '. The correct position of the needle was obtained by using a manual $\mathrm{K}$-wire drill, checking it by C.T-scan. The tip of the needle must be in a core of the lesion to obtain the maximum of thermoablation efficacy.

Results: All the cases had excellent results, with pain relive in $24 \mathrm{~h}$ and complete bone healing in three months. In two cases of chondrobalstoma, the symptoms disappeared while the healing of bone lesion is still in progress. Two complications was observed, a transient sciatic neuroapraxia and a "myalgia" of quadriceps recovered in 4 months. No case of recurrent lesions has been observed at a mean of 2 years follow up (min.1 years-max.4 years).

Conclusions: Mini-invasive thermoablation therapy of the Osteoid Osteoma is a gold standard in this benign bone lesion by now, overall in limb localizations. Even in other clearly benign lesions, this treatment could be performed; A histological examination must be performed and the two procedures can be made in the same session. Follow up have to be more frequent.

Significance: Enlargement of indication of thermoablation in benign bone tumors.

\section{3}

\section{TREATMENT OF THE PATHOLOGIC FRACTURES THROUGH UNICAMERAL BONE CYSTS IN CHILDREN AND ADOLESCENTS}

\author{
P. Šponer, G. P. Mathew, K. Karpaš, K. Urban
}

Purpose: The aim of this study was the evaluation of children and adolescents with pathologic fractures through unicameral bone cysts treated by operative procedures.

Methods: Fifteen boys and one girl with pathologic fracture through bone cyst operated on between 1999 and 2004 were included in this study. Twenty-two cases of pathologic fracture were identified in patients younger or equal to 16 years old $(2$ repeated pathologic fractures were recorded in 4 children and 3 repeated pathologic fractures in one patient). The primary cyst locations were the proximal humerus in 11 patients, the humeral diaphysis in 1 case, the proximal femur and the distal tibia in 2 cases each. The patients were followed up clinically for an average of 6 years (range, 18 months -9 years). The used treatment protocols were analyzed retrospectively and classified according to Dormans and Flynńs criteria (type I-IV). Radiological evaluation was based on the standard X-ray images taken at the latest follow up and assessed according to the classification system of Neer(excellent result, residuum, recurrence).

Results: Twenty pathologic fractures were primary treated conservatively (type II of treatment protocol), than steroid injection were successfully used in 4 children, delayed curettage of bone cyst and filling with autogenous or allogenous bone grafts or resorbable bioactive ceramics (Poresorb, LASAK, Prague) were performed in 10 children. Two pathologic fractures of lower extremity were treated primary by one stage surgery (type III of treatment protocol). There were performed osteosynthesis, curretage and filling of bone cyst with allogenous bone grafts. The clinical examination showed that all patients were free from subjective complaints. No inflammatory changes of soft tissue were found. Passive and active motions of the adjacent joints were possible in the full range and the treated limb retained its complete function in all patients. Radiological examination showed excellent results (no residuum or cysts recurrence) in 11 cases. A residual lesion was present in 5 patients with active unicameral bone cyst of proximal humerus.

Conclusions: The simultaneous operative treatment of the fracture and bone cyst used for lesions in weightbearing bones and the delayed specific treatment of the unicameral bone cyst after the fracture is healed (used in non-weightbearing bones) are our preferred treatment protocols for pathologic fractures through unicameral bone cysts in children and adolescents.

Significance: Different approaches to the operative management of the pathologic fractures though unicameral bone cysts can be 
used. Specific treatment of the bone cyst is usually delayed until the fracture is well healed. However, if the fracture is located in a weightbearing bone, the one-stage operative treatment of the fracture and lesion is required.

\section{4}

\section{SIMPLE BONE CYST TREATMENT WITH NORIAN SRS}

\section{J. De las Heras, P. Villanueva}

Purpose: Simple bone cyst is a benign lesion with a good spontaneous evolution, but with a risk of a pathologic fracture complication during childhood and adolescence. Several treatment methods have been advocated, some of them too aggressive for this type of lesion, and others not too resolutive in one, or at least few attempts of treatment. We have developed a new treatment, simple, safe and effective, for this type of lesions.

Methods: A prospective study has been developed in 20 patients with simple bone cysts treated with percutaneous filling with a remodelable bone cement (Norian SRS). The mean age was 11 years and most cysts were located in the proximal femur, proximal humerus and calcaneus, with a mean follow up of 4 years.

Results: The use of Norian SRS provided immediate structural support and good functional and radiographic results with a low aggressive technique. The postoperative recovery is fast and with minor symptoms. The radiological follow up shows progressive remodeling but with persistence of the cement. There is a learning curve to obtain a complete filling of the cyst and the rate of complications is low.

Conclusions: Norian SRS is a safe and useful method of treatment of simple bone cysts with a minimally invasive technique and with resolutive results. The advantages are the percutaneous procedure, the immediate structural support in one setting and throught the time, with progressive remodeling within the bone and the quick postoperative recovery.

Significance: This new method of treatment of symple bone cyst with the use of Norian SRS offers good results performed percutaneously, decreasing immediatly the risk of fracture and with progressive remodeling within the bone.

\section{5}

\section{THE EFFECT OF DILATION OF PEDICLES ON PULL-OUT STRENGTH OF THE SCREWS IN IMMATURE PIG MODEL}

\author{
G. Yilmaz, A. Alanay, C. Ozkan, G. Demirkiran, K. Daglioglu, \\ M. Pekmezci, M. Yazici
}

Purpose: Pedicle screws have become the implant of choice in spine surgery. Pull out strength of a pedicle screw increases as the diameter of the screw gets larger. Small pedicles of pediatric patients prevent the use of large size pedicle screws in this age group. As shown in an in vitro study one option to overcome this problem is to dilate and use larger screw in immature spine. But dilation reduces the pull out strength of pedicle screws. The aim of this study is to evaluate the biomechanical stability of screws placed in expanded pedicles and to compare with the non-expanded ones in an in vivo model and to investigate the effect of possible pedicle remodeling on screw stability.

Methods: Six-week old 2 pigs were used. After posterior subperiosteal exposure of lumbal segments $4.0 \mathrm{~mm}$ diameter and $20 \mathrm{~mm}$ long pedicle screws were placed on the left lumbal pedicles between L1and L2 (including L1 and L2). The right pedicles were dilated with stainless steel dilators with smooth surface until $4.2 \mathrm{~mm}$ and instrumented with screws of same dimension. At the end of three months the pigs were scarified and the pull out strength of each screw were measured.

Results: The pedicle screws were tested using pullout testing with Instron materials testing machine (Instron E 4301, Instron Ltd., Buckinghamshire, England). A constant rate of $1 \mathrm{~mm} / \mathrm{s}$ of pullout force was applied in line with screw. The average pull out strength of the expanded and non-expanded screws was $378.7 \pm 73 \mathrm{~N}$ (542-335) and $397 \pm 80 \mathrm{~N}$ (535-289), respectively. There was no statistically significant difference between the two groups $(P=0.54)$.

Conclusions: This study demonstrated that the biomechanical stability of the screws at the expanded and non-expanded pedicles is the same after three months. Even dilation of the pedicle decreases the pull out strength of the screw at the beginning; the pull out strength of both sides became equal after three months. The remodeling capacity of pedicles and overgrowth of bone on screws may have an effect on this result.

Significance: Dilation and using larger pedicle screws without scarifying their biomechanical stability might be an option for pedicle fixation in pediatric patient population. Even the patients may need an external support during immediate postoperative period they wont need external support after 3 months.

\section{6}

\section{PREOPERATIVE CORRELATION OF SAGITTAL, CORONAL, AND AXIAL DEFORMITIES TO PULMONARY FUNCTION IN AIS}

\author{
C. Johnston, B. S. Richards, D. J. Sucato, K. H. Bridwell, \\ L. G. Lenke, M. A. Erickson
}

Purpose: To determine the extent of respiratory impairment in severe idiopathic scoliosis, and identify ${ }^{\circ}$ of deformity in 3 planes which predict respiratory impairment.

Methods: 430 patients with Lenke types 1-4 scoliosis from the SDSG data base were evaluated. Preop measurements included: sagittal T5-12 Cobb; main thoracic curve Cobb; apical rotation (Nash-Moe). Standard preop PFT's (FEV1, FVC) were correlated with the X-ray measures, alone and in combination. FEV1 or FVC $<65 \%$ pred, T5-12 hypokyphosis $<10^{\circ}(\mathrm{HK})$; main thoracic scoliosis $>70^{\circ}(\mathrm{S} 70)$; Nash-Moe rotation 3 or $>$ (NM3) were defined as "severe" as opposed to "moderate".

Results: Sagittal: severe HK had mean FEV1 $=65.6 \%$ and $\mathrm{FVC}=71.4 \%$, while moderate $\mathrm{HK}$ had $78.4 \%$ and $82.2 \%$ respectively (both $\mathrm{p}<0.001$ ). In patients with FEV1 $<65 \%$, $35.7 \%$ were HK, while only $12.0 \%$ with FEV1 > $65 \%$ were HK $(P<0.00001)$. For FVC $<65 \%, 40 \%$ were HK, while only $12.4 \%$ with FVC $>65 \%$ were HK $(P<0.00001)$. Coronal: S70 had mean FEV1 $=66.7 \%$ and FVC $=71.7 \%$, compared to 78.3 and $82.3 \%$ respectively in non-S70 (both $\mathrm{p}<0.001$ ). $36.7 \%$ with FEV $1<65 \%$ had S70 scoliosis, only $13.6 \%$ with FEV1 $>65 \%$ had S70 curves $(P<0.001) .36 .0 \%$ with $<65 \%$ FVC had S70 curves vs. $15.2 \%$ with $\mathrm{FVC}>65 \% \quad(P<0.001)$. Axial 
$(n=412)$ : NM3 had mean FEV1 $=71.7 \%$ compared to $76.9 \%$ for non-NM3 $(P<0.03)$, with no difference in FVC between NM3 (77.8\%) and non-NM3 (80.8\%). NM3 was also n.s. between severe FEV1/FVC vs. moderate. Combining parameters: Patients with HK + S70 ("severe") deformity had mean FEV1 = 58.6\% and $\mathrm{FVC}=64.6 \%$ preop, compared to 80.7 and $84.3 \%$, respectively in non-HK $+\mathrm{S} 70$ ("moderate") cases (both $\mathrm{p}<0.001$ ). For $\mathrm{HK}+\mathrm{S} 70,69.2 \%$ have FEV $1<65 \%$ while only $12.5 \%$ with moderate have FEV1 $<65 \%(P<0.001)$. Similarly FVC $<$ $65 \%$ occurred in $53.8 \% \mathrm{HK}+\mathrm{S} 70$, but in only $8.7 \%$ of moderate cases $(P<0.001)$. Addition of NM3 to the HK + S70 tidemarks also produced significant differences in preop PFT's, this severity was observed in only $3 / 256$ patients.

Conclusions: Preop PFT impairment is significantly correlated with T5-12 HK $<10^{\circ}$ and main thoracic curve $>70^{\circ}$, alone and in combination. NM3 was less significant and rare $(n=3)$ in combination so its importance to PFT data is unclear.

Significance: Surgical attention particularly to "severe" sagittal and coronal deformities to improve pulmonary function is suggested by this data, but must await 2 year f/u PFT and X-ray data for confirmation.

\section{7}

\section{OPERATIVE TREATMENT OF HIGH-GRADE SPONDYLOLISTHESIS IN CHILDREN}

\section{G. Chan, D. Spiegel, J. Dormans}

Purpose: The purpose of this paper is to review our experience in the treatment of children with high grade spondylolisthesis comparing reduction and fusion with in-situ fusion.

Methods: A review of surgically treated cases of spondylolisthesis was reviewed from 1991 to 2006 . A total of 28 cases diagnosed with high grade spondylolisthesis were reviewed however only 17 cases were found to have adequate follow-up. Eight were treated with a reduction and fusion of the slip (group 1), 9 cases were treated with fusion in-situ (group 2). Radiographic parameters (slip angle, grade of the slip, lumbar lordosis and pelvic incidence) were evaluated. Clinical outcomes and complications on latest follow-up were also reviewed.

Results: On evaluation of radiographic parameters, correction of the slip angle and correction of the grade of the slip was superior in group 1. The correction was well maintained over time. The correction of lumbar lordosis was similar for both groups. Seven of eight cases $(87.5 \%)$ in group 1 were asymptomatic on followup, 3 cases developed post-operative neurologic deficits $(37.5 \%)$, two resolved without further problems while one case had persistent foot drop, two of these cases had documented pre-operative neurologic deficits. In group 2, 4 cases $(44 \%)$ had pain with activity on follow-up. Two cases $(22 \%)$ were noted to have a pseudoarthrosis and required revision surgery. Two cases $(22 \%)$ were seen to have progression of deformity.

Conclusions: In conclusion, excellent correction of sagittal balance was seen with the reduction group. This correction was well maintained over time. High grade spondylolisthesis can be treated safely with a reduction and fusion with relatively low complication rates.

Significance: Controversy still exists regarding the optimal treatment for children with high grade spondylolisthesis. This study compares outcomes and describes our treatment protocol to treat this difficult surgical problem to determine optimal management in children with high grade spondylolisthesis.

\section{8}

\section{MEP/SSEP MONITORING IN SPINAL DEFORMITY SURGERY: INCIDENCE AND FACTORS LEADING TO SIGNIFICANT ELECTROPHYSIOLOGICAL EVENTS}

\author{
M. G. Vitale, D. Moore, D. P. Roye, H. Matsumoto, \\ J. A. Gomez, J. E. Hyman
}

Purpose: Despite advances in surgical technique, neurological injury remains a potentially devastating complication of spinal deformity correction surgery. The purpose of the study is to describe surgical and patient factors associated with "electrophysiologic (EP) events" and neurogenic deficits.

Methods: A retrospective chart review, looking at "EP events" during surgery, was conducted on 162 patients who received surgical treatment of their pediatric spine deformity from 1999 to 2004. Results: Ninety three percent of cases $(n=151)$ were successfully monitored by either somatosensory evoked potential (SEP) or motor evoked potential (MEP) monitoring. All three neurologic deficits that occurred in this study cases were successfully detected by $\mathrm{EP}$ monitoring $(0.02 \%, \mathrm{p}=0.002)$. In those 151 cases that were successfully monitored, "EP events" were occured in twenty $(13.2 \%)$ cases. The most common cause was systemic change $(45 \%)$ and curve correction $(40 \%)$. In those 20 cases, when corrective actions were made $(n=15)$ "EP events" reversed to baseline values in all cases. When no corrective actions were taken $(n=5)$ there was no reversals of "EP events" to baseline. Patients with kyphosis had a trend toward significantly higher rates of "EP events" $(P=0.174)$ and patients who had cardiopulmonary co-morbidities had significantly higher rates of "EP events" $(P=0.007)$.

Conclusions: MEP and SSEP monitoring are valuable tools for the spine surgeon during scoliosis correction. MEP monitoring, although less likely to be successful than SEP monitoring for all types of scoliosis, is still successful in the majority of cases. A "significant EP" change likely represents a neurologic insult that may lead to a postoperative neurologic deficit. A "corrective action" in response to a "significant EP change" has a high likelihood of reversing that EP change and failure to make a "corrective action" is likely lead to "significant EP changes" that remain abnormal at the completion of the case.

Significance: Consistent with existing literature, the EP monitoring was successful in the vast majority of deformity surgeries. "EP events" were able to be reversed with corrective action and to predict neurologic deficits. Our study found that patients with kyphosis and/or cardiopulmonary co-morbidities have higher risk of significant "EP events" during the surgeries.

\section{9}

\section{USE OF THE VACUUM ASSISTED CLOSURE IN PEDIATRIC SCOLIOSIS PATIENTS WITH DEEP SPINAL INFECTIONS}

\section{F. Canavese, J. I. Krajbich, K. Emara, S. Gupta}

Purpose: Post-operative deep infections are still relatively common in instrumented spinal deformities of children affected by spinal dysraphism or other chronic debilitating conditions and they can result in significant morbidity, costs, and compromise of the desired correction. The purpose of the present study is to review the 
efficacy of the wound Vacuum-Assisted Closure system (VAC) in the treatment of early onset deep spine infection following spinal instrumentation and fusion in children and adolescents.

Methods: 14 patients (10 girls, 4 boys) with deep post operative spinal infections treated with VAC were reviewed. All patients had inflammatory marker evaluation, intraoperative culture, thorough lavage, removal of all macroscopic contamination, devitalized tissue and loose bone graft and application of VAC. Patients received antibiotics for a period of at least 6 weeks based on the results of the wound culture sensitivities. Length of follow-up, need for hardware removal, infection eradication, spinal pseudoarthrosis, need for additional surgery after VAC application, and any loss of correction or hardware loosening were evaluated.

Results: The average age was 13.4 years (range 3-19). Twelve patients had thoracolumbar curvatures, one had an isolated thoracic curve and one had kyphosis. Combined anterior release and posterior fusion was performed in 7 patients, and posterior fusion alone in 7. Standard segmental instrumentation was used for non neuromuscular curves and segmental instrumentation into the pelvis for most of the neuromuscular curves. Allograft was used to in 13 of 14 patients. The average surgical time was 417 min (range 124-715). The average number of levels fused was 10 (range 6-16). All patients presented with discharge from part of the wound and constitutional symptoms were present in $57 \%$ of cases. No patients had exposed hardware at the time of initial presentation. The VAC was applied at initial surgical debridement in 10 out of 14 patients. The length of application ranged from 5 to 42 days (average 21 days) and the VAC dressing was changed twice weekly on average. The average follow up was 3.6 years months (range 2-6). The average number of surgeries required for change of VAC was 3.4 (range 1-10). All patients successfully completed their wound VAC treatment regime. None required removal of the main instrumentation, had significant loss of correction or had recurrence of the infection. The laboratory indices have normalized in all patients.

Conclusions: Wound VAC technique is a useful tool in the armamentarium of the spinal surgeon dealing with patients susceptible to wound infections, especially those with neuromuscular diseases. It allows for retention of the instrumentation and maintenance of the spinal correction. It's reliable and easy to use.

\section{0}

\section{PROSPECTIVE COMPARISON OF SUPINE BENDING, PUSH PRONE, AND TRACTION UNDER GENERAL ANESTHESIA RADIOGRAPHS IN PREDICTING CURVE FLEXIBILITY AND POSTOPERATIVE CORRECTION IN ADOLESCENT IDIOPATHIC SCOLIOSIS}

\author{
R. W. Liu, A. L. Teng, C. Poe-Kochert, D. G. Armstrong, \\ G. H. Thompson, J. P. Son-Hing
}

Purpose: Preoperative supine side-bending radiographs have been recommended for routine use in Adolescent Idiopathic Scoliosis (AIS), to guide selection of fusion levels and to classify curves. The purpose of this study was to prospectively compare the flexibility of side bending, push prone, and traction under general anesthesia (UGA) radiographs.

Methods: Fifty-four consecutive AIS patients with 47 main thoracic (MT) and 13 thoracolumbar/lumbar (TL/L) structural curves were studied prospectively. Mean age was $14.2 \pm 1.9$ years. All patients had PA/lateral standing, AP supine bending, PA push prone and AP traction radiographs UGA preoperatively. The latter were obtained by manual traction via the head and ankles. All patients had a posterior spinal fusion (PSF), while two patients also had an anterior release. Cobb angles were measured, and ratios calculated between the flexibility radiographs and the preoperative radiographs to determine curve flexibility, and between the standing postoperative and the preoperative radiographs to determine correctability. Comparisons were performed using twotailed $t$ tests.

Results: The flexibility ratios for supine bending, push prone, and traction UGA for MT curves were 0.49, 0.36, and 0.54, while the postoperative correctability ratio was 0.64 . Larger values indicate greater flexibility or correctability. For MT curves there was no significant difference between supine bending and traction UGA. Both demonstrated significantly greater flexibility than push prone. Ultimate correction was significantly greater than that expected from any of the preoperative flexibility radiographs. The flexibility and correctability ratios for the TL/L curves were 0.61 , $0.43,0.60$, and 0.62 , respectively. For the $\mathrm{TL} / \mathrm{L}$ curves, there was no significant difference between flexibility shown by supine bending and traction UGA. Push prone was significantly less effective. Ultimate correction could be estimated from supine bending and traction UGA.

Conclusions: Traction UGA and supine bending radiographs demonstrate more flexibility than push prone radiographs in AIS. Surgical correction of MT curves cannot be predicted by these preoperative flexibility radiographs. For TL/L curves correction can be estimated with traction UGA and supine bending radiographs.

Significance: Traction UGA and supine bending demonstrate more flexibility than push prone radiographs. None of our flexibility radiographs were able to predict postoperative correction in MT curves, while traction UGA and supine bending did estimate postoperative correction in TL/L curves. Traction UGA films show both structural and compensatory curves on the same radiograph and therefore may provide an estimate of ultimate spinal balance.

\section{1}

\section{FACIAL ASYMMETRY ANALYSIS IN CHILDREN WITH CONGENITAL MUSCULAR TORTICOLLIS AFTER OPERATIVE TREATMENT}

A. Masoń, A. Grzegorzewski, E. Zmysowska, I. Pieszyński, J. Dziewierz, M. Synder, R. Lebiedziński

Purpose: Congenital muscular torticollis is caused by fibromatosis within the sternocleidomastoid muscle and is seen in $0.3-$ $0.5 \%$ of all live births. In spite of frequent checkup this congenital malformation is not diagnosed in neonates in every case, what is the reason of delayed conservative and operative treatment? Late treatment can cause malocclusion, deformities of vertebrae and facial asymmetry. The aim of this study was a digital analysis of facial asymmetry in children with congenital muscular torticollis.

Methods: The study group consisted of 16 patients with congenital muscular torticollis treated surgically by sterno-cleidomastoideus myotomy at the age of 1-35 years (mean age 5.7 years), the follow up was performed from 2 to 26 years after the surgery (mean 13,4 years). The control group consisted of 13 persons age 7-29 years with no torticollis or other dysfunctions connected with facial asymmetry. Both groups were matched with regard to age and gender. En face photographs of every person were taken and analyzed by mean of Facial Asymmetry 
Index computer program created for this study. Three observers independently matched 12 characteristic points on every photo - six on the right and six on the left side of analyzed faces (medial and lateral angle of the eye, most lateral part of wing of the nostril, angle of the mouth, lower attachment of an auricle and angle of the mandible). On the basis of their position, average horizontal deviation, average vertical deviation, maximum horizontal deviation and maximum vertical deviation indexes were calculated. Additionally facial asymmetry in patients operated before and after age of 3 was compared. We employed the following methods for statistical analysis: Wilcoxon test, Shapiro-Wilk test, measures of central tendency, variability.

Results: Facial average horizontal deviation (AHD) and maximum horizontal deviation (MHD) in study group were statistically significantly less than in control group (average AHD 2,288 vs. 2,586 asymmetry index [ai] with confidence $90 \%$; average MHD 7,22 vs. 26,28 [ai] with confidence $95 \%$ ). There were no statistical significances of average and maximum vertical deviations between groups. There was no statistical significances of facial asymmetry in children treated surgically before and after age of three, but the average of AHD and MHD were less in study group (2,52 and 21,18 vs. 2,6525 and 31,38 [ai]).

Conclusions: (1) Facial asymmetry caused by muscular torticollis is permanent. (2) Facial asymmetry concerned mainly horizontal axes. (3) Facial asymmetry concerned mostly children operated after 3 years of age, but the differences are not statistically significant.

Significance: Children with muscular torticollis should have regular follow up, to prevent further deformation.

\section{2}

\section{INTRAMUSCULAR HYDATID CYSTS PRESENTING AS UNUSUAL SOFT-TISSUE MASSES IN CHILDREN}

\section{B. Erol, B. Kocaoglu, H. A. Akgulle, O. Ofluoglu}

Purpose: In this prospective study, we aimed to determine important factors in diagnosis and present a standard treatment regimen for this rare entity.

Methods: Between 2000 and 2005, in two different hospitals, nine children with a diagnosis of intramuscular hydatid cyst were treated. The presenting symptom was a painless growing mass that has been recognized in last three to six months. The location of the lesions was the thigh-different compartments (4), calf-gastrocnemius muscle (2), low-back-paraspinal muscles (2) and backinfraspinatus muscle (1). Physical examination of the lesions usually revealed a moderate/large, deep-seated, fluctuant mass, mostly without well-defined margins. Radiological evaluation of the masses was mainly performed by magnetic resonance imaging (MRI), but all patients had plain radiographs and ultrasonography of the involved area as well. Chest, abdomen and cranial computed tomography (CT) studies were also done to search for hydatid cysts in other locations. Specific antibodies were studied by serological tests. Aspiration or biopsy was not performed in order not to spread cyst contents into the systemic circulation. The lesions were excised with wide surgical margins and all patients received adjuvant albendazole treatment $(10-15 \mathrm{mg} / \mathrm{kg}$ day $\left.{ }^{1}\right)$ in the pre- and post-operative period. All patients had regular clinical and radiological (with MRI) follow-up postoperatively; the average follow-up was 36 months (range 29-81 months).

Results: MRG was diagnostic in all cases; due to the developmental stage of the disease different morphological features and signal intensities was observed on MRG. Ultrasonographic examination demonstrated cystic nature of the masses and supported the diagnosis of intramuscular hydatid cyst. Plain radiographs were not diagnostic. In two patients cranial CT scan revealed asymptomatic intracranial cysts; these two patients initially had neurosurgical procedures, followed by excision of the intramuscular lesions. Serological tests were positive only in three cases. None of the patients had perioperative (spreading of the cyst contents) or postoperative (neurovascular damage, infection) complications. There was no recurrence. All patients returned to their preoperative activity level within four weeks, without any significant morbidity.

Conclusions: The preoperative radiological evaluation, particularly MRG, is very important to avoid a biopsy or to prevent improper intraoperative management of the intramuscular hydatid cysts. The same surgical principles as for malignant tumor resection must be employed for intramuscularly located hydatid cysts, and an attempt made to achieve wide surgical margins in order not to spread cyst contents into the systemic circulation. Significance: The same surgical principles as for malignant tumor resection.

\section{3}

\section{SHORTER ANTIMICROBIAL TREATMENTS FOR CHILDHOOD SEPTIC ARTHRITIS}

\section{Pääkkönen, H. Peltola, M. Kallio, P. Kallio}

Purpose: In a prospective study, to find out the optimum duration of antimicrobial treatment in childhood septic arthritis. In a retrospective setting, to reevaluate the indications for surgery (arthrotomy, arthroscopy).

Methods: 130 children at age 3 months to 15 years with culturepositive (joint and/or blood) septic arthritis were randomized to receive antimicrobials for 10 versus 30 days. Except initially for $2-$ 4 days intravenously, medication was oral. We did not perform routine arthrotomies/arthroscopic lavages. Indication for surgery was evaluated individually in cases with unusually slow response to antimicrobial treatment. The follow-up lasted for at least a year.

Results: Sixty-three children were in the short, 67 in the long treatment group. More extensive surgery than percutaneous joint aspiration was executed only in 20 patients (15\%). All children made full clinical and radiographic recovery without any late sequelae, irrespective of the duration of antimicrobials and whether surgery was performed or not. One patient in the longer treatment group had recurrent septic arthritis, with no permanent joint damage.

Conclusions: Large doses of well absorbing oral antimicrobial (clindamycin, first generation cephalosporin, or ampi/amoxicillin) administered for less than 2 weeks, starting intravenously, suffice in most cases of uncomplicated childhood septic arhritis, provided the clinical response is good and the C-reactive protein normalizes. Routine arthrotomy or arthroscopic lavage is not recommended.

Significance: Shorter antimicrobial treatments and less surgery than current recommendations can guarantee similar results in uncomplicated childhood septic arthritis with a reduction in treatment costs. Especially if the result can be transformed to developing countries, this would provide improved treatment in a setting where only scarce resources are available. 


\section{4}

\section{SUBACUTE OSTEOMYELITIS OF THE PATELLA}

\author{
T. Terebessy, G. Szőke, M. Szabó, S. Kiss
}

Purpose: Subacute osteomyelitis accompanied by slight complaints and diverse radiological manifestations usually causes diagnostic problem. Radiological signs are not eligible for definite diagnosis of an osteolytic lesion of the patella. Previous studies about subacute osteomyelitis of the patella are not found in the literature. The aim of this study was to assess the clinical and radiological signs that are used to confirm diagnosis and to evaluate the efficacy of therapy in patellar subacute osteomyelitis. Methods: Fever, laboratory parameters, sclerotic margin on the $\mathrm{X}$-ray and the penumbra sign on the T1 weighted MRI images were assessed in 6 cases with anterior knee pain due to a small osteolytic lesion in the patella. Excochleation, bacterial analysis and hystological evaluation were done in all cases. $3 \times 300 \mathrm{mg}$ clindamycin or $2 \times 500 \mathrm{mg}$ cefuroxim was administered for 6 weeks after surgery.

Results: No fever occured and inflammatory laboratory parmaters were within normal range in all cases. 4 of the lesions had sclerotic margin on the X-ray. The penumbra sign were present in 3 cases on the T1 weighted MRI images. Microbiological analysis was negative in all cases. Subacute osteomyelitis was diagnosed by hystology, based on the presence of polymorphonuclear leukocites and lymphocites in fibrous tissue. Excochleation and - even in the absence of positive bacterial culture - 6 weeks of antibiotic treatment resulted in complete clinical remission in all 6 cases.

Conclusions: The usefulness of the radiological signs is questionable, surgery and histology is essential for the correct diagnosis of subacute osteomyelitis of the patella. Treatment includes debridement and postoperative antibiotics.

Significance: Subacute osteomyelitis of the patella is a rare cause of anterior knee pain; however consequent complaints should lead to X-ray imaging where the presence of an osteolytic lesion should be followed by surgery.

\section{5}

\section{CONGENITAL RADIOULNAR SYNOSTOSIS: A NEW OPERATIVE TECHNIQUE FOR OBTAINING FUNCTIONAL SUPINATION-PRONATION RANGE, A REPORT OF TWO CASES}

\section{A. S. Allam}

Purpose: Evalution of the degree of forearm rotation improvement with this new operative technique.

Methods: Two children; 5 and 6 years old respectively with CRUS , Wilkie type I, with fixed full pronation deformity were managed by the new ALLAM'S OPERATION which is a one stage intervention including separation of the bony fusion, with special cementation technique of the ulnar side of the osteotomy, double osteotomy of the radius and a single osteotomy of the ulna (all of the 3 osteotomies were done percutaneously) with intramedullary $\mathrm{K}$. wire fixation of osteotomies at the mid-prone position and above elbow casting for 6 weeks.

Results: Excellent significant functional range of forearm rotation was obtained with no significant complications after a follow-up period of 28 and 15 months, respectively.
Conclusions: A functional supination-pronation range could be obtained in cases with congenital radioulnar synostosis.

Significance: This technique might be a hope to obtain a functional supination-pronation range in cases with congenital radioulnar synostosis.

\section{6}

\section{THORACIC OUTLET SYNDROM IN CHILDREN}

\section{Mickel, G. Weigel, W. Girsch}

Purpose: According to literature thoracic outlet syndrome (TOS) is not to be seen before the second decade of life. We report two cases of 11 year old girls suffering from TOS due to cervical ribs bilaterally.

Methods: Starting at an age of 9 years the symptoms of painful ulnar nerve entrapment in one case and painful shoulder and upper arm syndrome in the other case were iatrogenically neglected due to normal electrophysiological values. Two years later an investigation in our department yielded the diagnosis of TOS, based on the finding of cervical ribs and stenosis of the subclavian arteries. Consecutively the resection of the right cervical rib was performed in both patients. Due to increasing symptoms on the left side the procedure was repeated six months later in one patient.

Results: The resection of the cervical rib was performed successfully via single supraclavicular incision in all cases. Procedure and postoperative course were without complications, with complete preserved motor function and transient slightly reduced sensory function. Both girls are pain free and without any symptoms of nerve compression.

Conclusions: TOS is well documented in literature, focusing on clinical symptoms as the main indication for surgery. TOS in children has not been reported up to now, but we could confirm the clinical diagnose by radiological findings. The presented cases of our girls show, that "nerve pain" must not be neglected in children.

\section{7}

\section{CONGENITAL RADIAL CLUB HAND; LENGTHENING BY A MONO-PLANE DEVICE, IN TYPE IV PATIENTS WITH PREVIOUS WRIST CENTRALIZATION}

\section{A. S. Allam}

Purpose: Evaluation of the results of bone lengthening in congenital radial club hands using a mono-plane fixator.

Methods: Eight patients with type IV congenital radial club hand-with previous wrist centralization-were operated upon. The patients' average age was 6 years. Average ulnar shortening was $6.2 \mathrm{~cm}$ with mean angular deformity of $40^{\circ}$. A one stage procedure including acute correction of the deformity via an ulnar distal closing wedge osteotomy and the application of a mono-plane lengthening device was done. Lengthening was started - from the osteotomy site - 10 days later.

Results: Ulnae were lengthened $30-62 \%$ of their original length with a healing index of 36 days in average (range 28-43 days). The angular deformity was completely corrected in all patients; with mild recurrence of the deformity in one patient later during late follow up. 
Conclusions: This one stage procedure of correction of the deformity and lengthening of the ulna using a mono-plane lengthening device is a simple technique with good results and few reported complications.

\section{8}

\section{SCREW EPIPHYSEODESIS AROUND THE KNEE (AXIS AND LENGTH CORRECTION)}

\section{P. Marschalkó, M. K. Szabó}

Purpose: The aim of the study is to evaluate the effectiveness of screw epiphyseodesis around knee.

Methods: Bicondyler distal femoral epiphyeodesis was performed in 11 patients three of which had bicondyler epiphyseodesis of the tibia at the same time (Group A) and unicondylar distal femoral epiphyseodesis in 31 patients (Group B). In Group A we detected the limb length discrepancy in millimeters on scanogram before the surgery and after the removal of screws. In Group B we mesured the angle between the knee joint line and the longitudinal axis of the femur (degree of the valgus deformity) evaluating the change from the beginning to the removal of screws and 1 year later.

Results: Axis correction rate was $0,39^{\circ}$ per month (average of total change was $8^{\circ}$ ) and in all cases valgus deviation of the knee was observed. There was no epiphyseal arrest after the removal of screws. The planned amount of the correction was performed in every case. Slight recidivation was observed 1 year later (approximately $2^{\circ}$ ). The length correction rate was $0.56 \mathrm{~mm}$ per month. In spite of the late registration of these patients half of the total limb length discrepancy was corrected (average discrepancy $30 \mathrm{~mm}$ ). We removed all screws after the skeletal maturity. Axial deviation of the femur and/or the tibia or overcorrection was not observed.

Conclusions: The screw epiphyseodesis is a minimal invasive, safe and easy method to correct the limb length discrepancy and the axial deviation of the femur and/or the tibia. The timing of these procedures is the most important factor. This can lead the patient to complete correction or can detain our effort. We tend to apply new methods e.g., the eight plate technique but the low cost is a telling argument.

\section{9}

\section{MOLECULAR ANALYSIS IN PFFD PATIENTS}

\section{Frydrychová, D. Tvrdík, J. Chomiak, P. Dungl}

Purpose: To study gene expression of osteogenesis and angiogenesis in physiological and pathological bone tissue (pseudoarthrosis in PFFD patients) and identify the differences in these processes and their consequences.

Methods: The piece of physiological or pathological bone was taken during the elective surgical procedure after the inform agreement of the patient. The specimen was put to the RNAse free water and was frozen. The RNA was isolated and prepared according to protocol for oligoarray analysis (SuperArray Bioscience Corporation). 113 genes of osteogenesis and 113 genes of angiogenesis were analysed.

Results: The differences in quantity and representation of the genes were noted. Some genes were considered as over-expressed in PFFD tissue vs. control (f.e. the gene for calcitonin receptor, collagen XII, collagen I alpha 2, collagen II, collagen IX, FGFR2, fibronectin, integrin) and some of the genes as under-expressed (f.e. the gene for annexin A5, collagen XVIII alpha1, collagen I alpha1, cathepsin K, FGFR1, FGFR3, IGF2, VEGFB) In general, in PFFD tissue were expressed less genes of osteogenesis and especially angiogenesis comparing with physiological tissue.

Conclusions: The study is still running. The other analyses (Real Tome PCR, Western blot, micro dissection) are designed for the next years. Also the analysis of parents' DNA (in PFFD patients) by $\mathrm{CGH}$ (comparative genomic hybridization) is on the program. Significance: Statistic methods have not been used yet, the study is still running.

\section{0}

\section{EXPERIMENTAL STUDY OF MECHANICAL EFFECTS IN THE PATHOGENESIS OF AMNIOTIC BAND SYNDROME IN THE RABBIT}

\author{
C. Amat, C. García Fontecha, F. Soldado, J. Nardi, J. Sevilla, \\ M. Aguirre
}

Purpose: Amniotic band syndrome is a congenital pathology characterized by limb constrictions. The disorder lacks precise definition, and its exact pathogenesis is unknown. Though there have been theories advanced to explain the origin of the condition, none have been scientifically validated. The "exogenous" theory, popularized by Torpin, is the most widely accepted. It suggests that early amniotic rupture leads to the formation of amniotic strands, which then induce extremity bands and other abnormalities. In this disorder, histological examination of the excised bands demonstrates them to be composed of fibrous tissue. Our hypothesis is that annular external compression in a fetal lagomorph (rabbit) limb will not produce a band of subcutaneous fibrous tissue characteristic of amniotic band syndrome as the fetus develops. Methods: We operated on one limb of 10 different lagomorph fetuses, each at 21 days of gestation. The extremity was ligated with a nylon suture at the infracondylar level. At 30 days gestation, each fetus was delivered by caesarean section. The mechanically constricted zone and the limb distal to it were analyzed histologically using different techniques.

Results: Histological analysis did not show subcutaneous fibrous tissue in the mechanically constricted zone. The distal segment showed dilatation of lymphatic vessels and edema of soft tissue. Conclusions: By applying an external band, we cannot induce formation of tissue characteristic of amniotic band syndrome, as would be expected according to the exogenous theory of the syndrome's etiology.

Significance: No fibrous tissue was developed, and therefore this experimental study does not support a mechanical exogenous etiology for amniotic band syndrome.

\section{1}

\section{TEMPORARY HEMIPHYSEAL STAPLING FOR THE TREATMENT OF GENU VALGUM IN CHILDREN}

\section{T. Kučera, K. Karpaš, P. Šponer}

Purpose: A moderate degree of genu valgum is a physiologic finding in children 2-6 years of age. Sometimes genu valgum persists or increases in preadolescent years and it can cause 
functional and cosmetic problems. We evaluated the results of temporary stapling of the medial femoral physes in children with genu valgum.

Methods: This retrospective study evaluated pre- and post-operative findings in children following to the skeletal maturity. The condition was assessed by measuring clinically the intermalleolar distance and radiologically the tibiofemoral angle and the mechanical axis. The indications for the surgery were patients complaints in children older than 11 years of age for girls and 12 years of age for boys, the intermalleolar distance more than $8 \mathrm{~cm}$ and the lateral position of the mechanical axis. The surgery consisted in temporary stapling of the medial femoral physes following Blounts technique by three staples. The staples were removed when the position of the mechanical axis was physiologic. We followed the children to the skeletal maturity and we evaluated possible complications including rebound phenomenon. Results: We evaluated 46 patients (92 knees), 24 boys with an average age at the time of the surgery 13 years and 22 girls with the average age 12 years. All children had gait disorder; they were easily fatigued with an irregular pain in the thigh and calf. 16 children $(35 \%)$ had patellofemoral complaints. The intermalleolar distance was on the average $10.4 \mathrm{~cm}$ and the tibiofemoral angle was on the average $11.6^{\circ}$ before the surgery. We let full weightbearing between second and third week after surgery. The staples were removed on the average 10 months after the surgery. As for complications there was one knee infection, one mechanical failure of the staples with necessity of reimplantation, both with good results. 42 children were satisfied with improvement in gait and with a decrease in clinical symptoms. Four children observed during 1.5 year after staples removal the recurrence of the valgus deformity on the average to $45 \%$ of the initial value. No premature physeal closure was observed.

Conclusions: Temporary hemiphyseal stapling for the treatment of the genu valgum is less magnitude procedure with early weightbearing and satisfactory results. Rebound phenomenon was the only problem in four children.

Significance: Temporary hemiphyseal stapling for the treatment of the genu valgum in children is a reliable method with satisfactory results and few complications.

\section{2}

\section{PELVIC DEFORMITY IN RECURRENT BLADDER EXSTROPHY}

\section{Rogalski, J. Zacher, K. Fliesenberg, M. Deja}

Purpose: We saw a 3 years old Libyan girl with congenital bladder exstrophy. The incidence is $1 / 40,000$ live births. These girl suffered from recurrence after $2 \times$ proceeding OP in Libya (1. turn-in-OP, 2. cerclage of symphysis).

Methods: The patient presented a limited walking distance, early fatigue and typical waddle gait with external food progression and external rotation of the hip, reduced IR und widened pelvic shape. There was an exstrophy of a hypoplastic bladder, incontinence and anamnestic recurrent infections. The musculosceletal deformities: the symphysary diastasis was $6.5 \mathrm{~cm}$ with anterior unstable pelvic ring, shortening of pubic rami $(30 \%)$ and abnormal external rotation of posterior pelvic segment. There was a bilateral acetabular retroversion and diastasis of abdominal chest muscles. Our aim was the repair of pelvic deformity as a prerequirement for secure genitourinary reconstruction-procedures and normalisation of gait and improvement of childs mobility. 1st step: Realisation of a Mainz-Pouch - impossible turn-in of an insufficient hypoplastic bladder (volume $<50 \mathrm{ml}$, hypertrophy wall $>2 \mathrm{~cm}$ ).
Second step: Orthopedic treatment with bilateral anterior horizontal innominate osteotomy and bilateral posterior derotating vertical iliac osteotomy according to Sponseller and genitourinary reconstruction. Postoperative immobilization was achieved by Buck-Skin-Extension. 1 week later we started with an additional approximation of symphysis via external fixator. After 6 weeks we found a consolidation of osteotomies with a symphyseal distance of $1.0 \mathrm{~cm}$. A pin infection was successfully cured with antibiotics. The external fixator was removed and symphysis was augmented with an autologue semitendinosis-plasty. The mobilization started in the 7 th week.

Results: We achieved day continence with intermittent catheterization via Mainz-Pouch, free standing and gait with parallel food progression, full active and passive ROM of hip joints. There was no sign of recurrent symphyseal diastasis. So she could return to her country. In the meanwhile we treated two other children in the same manner.

Conclusions: In cases of recurrent bladder-exstrophy we recommend an interdisciplinary approach of treatment. It includes the reconstruction of a continent neo-bladder, abdominal wall closure and three-dimensional correction of pelvic deformity (via bilateral anterior horizontal innominate- and posterior derotating vertical ilium - osteotomy with consecutive approximation of symphysis) as a long-lasting solution.

\section{3}

\section{CONGENITAL DISLOCATION OF KNEE}

\section{Y. A. Rady}

Purpose: Congenital subluxation and dislocation of the knee is a rare event with incidence estimated to be about $1 \%$ as common as developmental dysplasia of the hip, it is usually recognized at birth, but it can diagnosed prenatally by ultrasound. The first description of this event was by Chanssier in 1812 and Chatelaine in 1822 , but the first report in the English literature was by Shattock in 1891. Its etiology is not fully discovered, it is probably partly genetic and partly developmental due to extrinsic mechanical factors, the causes believed to be responsible include; congenital absence or hypoplasia of cruciate ligaments, arthrogryposis, laxity combined with intrauterine breech position, and paralysis of the knee flexors. The dislocation is anterior one, and may be associated with other abnormalities. The purpose of this work was to investigate the results of manipulation and splinting in newly borne cases, and the surgical reduction with new reconstructive procedure to the extensor knee mechanism.

Methods: Five cases with 9 knees were managed by different methods according to their age and grades. Two cases were treated, during the first week of life, by repeated manipulation to flex the knees. The other 3 cases ( 5 knees) their surgical treatment were begun after 3 years by releasing the quadriceps tendon from the proximal patellar pole and the retinaculae from both vasti, the anterior knee capsule by transverse incision, flex the knee, and reconstructing a new extensor knee mechanism.

Results: The results obtained were reduced stable knees, with flexion range above $120^{\circ}$, and full active extension without lag in all cases.

Conclusions: The treatment of CDK in the first week postnatal conservatively is successful. In neglected cases surgical release is mandatory, and with this reconstructive procedure to the extensor knee mechanism full active extension can achieved and the child walk unsupported. 
Significance: The use of malleable aluminum splint facilitates the care of the baby and allows repeated manipulation into more flexion every 3 days. As a result, these will shorten the period of treatment. The use of these reconstructive procedures is essential to overcome the wide gap present after anterior release and flexion of the knee in neglected cases.

\section{4}

\section{ARTHRODESIS OF THE HIP: AN OPTION IN THE TREATMENT OF SEVERELY DAMAGED HIPS IN ADOLESCENTS AND YOUNG ADULTS}

\section{K. Lisiecka, J. Czubak}

Purpose: Aim of the study is to present the arthrodesis as a method of management of severely damaged hips in adolescents and young adults.

Methods: Records of 16 patients operated in our hospital were reviewed. The age at surgery ranged from 12 to 26 years. Arthrodesis of the hip was performed in these patients because of pain, significant dysfunction of the limb caused by contractures and limited range of motion in the hip. The source of this malfunction was always a severe deformation of the joint surfaces, which made any other surgical solution very difficult to apply. No matter what the etiology of the deformity was (DDH, neonatal septic arthritis, traumatic lesions), an intraarticular or combined intra- and extraarticular arthrodesis was performed. Plaster cast immobilization was applied in all cases. Follow-up ranged from 6 months to 4 years. Harris hip score and Womac scale were used to evaluate the results.

Results: In all our patients the results were similar to those observed after hip replacement.

Conclusions: The artrodesis of the hip may be considered as a treatment option for severely damaged hips in young patients for whom total hips replacement is impossible or controversial.

\section{5}

\section{STAHELI ACETABULAR SLOTTED AUGMENTATION: RESULTS OF TREATMENT}

\section{Shadi, A. Koch, M. Napiontek, P. Koczewski}

Purpose: To present the result of Staheli procedure in the treatment of femoral head coverage deficient due to different etiology. Methods: We analyzed 8 patients at the age of 7-20 years (mean 12.2) in whom 10 hips were treated because of hip dysplasia (3), Perthes disease (3), pseudoachondroplasia (2), multiple epiphyseal dysplasia (1), and femoral neck exostosis (1). Follow-up time was 3.3 years (1-6). The evaluation includes the analysis of the X-ray of the hip joints in a-p view before operation, immediately after surgery and at follow-up. The relation of the femoral head to the acetabular was evaluated in all X-ray by Wiberg angle (CE), acetabular index (AI), Reimers migration index (RM). The range of hip joint motion at the same time was also evaluated.

Results: Mean Wiberg angle improved from $17^{\circ}$ before augmentation procedure ( -10 to 26 ) to $42^{\circ}$ after operation and at the last follow-up (28-63). Significantly less changes observed in acetabular index in which the mean angle was improved from $29^{\circ}$
(16-47) to $21^{\circ}(9-50)$, and in the last follow-up to $22^{\circ}(9-50)$. In two cases this angle was deteriorated by $4^{\circ}$ and $6^{\circ}$. The most significant changes were observed in the range of RM index in which the mean value before treatment was $40 \%$ (29-59), after treatment decreased to $6 \%(0-22)$ and in the last follow-up to $11 \%(0-29)$. In all patients no hip range of motion deterioration observed after surgery and in the last follow-up. There was no statistically relationship observed between the improvement of radiological parameters and patient age at operation or the etiology.

Conclusions: Acetabular slotted augmentation of Staheli procedure is effective method to improve the non spherical femoral head coverage because of different etiology. The most sensitive radiological parameter indicating changes in the femoral head coverage were Reimers migration index and $\mathrm{CE}$ angle of Wiberg.

\section{6}

\section{NAIL FEMORAL LENGTHENING IN CHILDREN}

\section{Bonnard, J. Perlès, Y. prezelin}

Purpose: Gradual limb lengthening with currently used external fixation techniques can result in less optimal outcomes, with complications including infection, stiffness of adjacent joints, and secondary axial deviation of the extremity. The Albizzia nail seems to be an alternative to external fixation for progressive lengthening of the femur. We have analyzed the results and complications obtained with this nail on 17 femur.

Methods: Between 1997 and 2006, 17 femora (14 patients) underwent limb lengthening with an Albizzia nail to treat a congenitally short extremity ( 8 patients), post-traumatic limb-length discrepancy ( 5 patients), post-infection limb-length inequality ( 3 patients), or developmental problems (1 patient). Eleven patients (11 femora) underwent unilateral surgery to equalize the limb lengths, and 3 (6 femora) underwent bilateral surgery to correct short stature. The mean age was 16 years and one month (range, 13-18.5 years). After intramedullary corticotomy of the diaphysis of the femur, an Albizzia nail was inserted in an antegrade fashion. Fifteen alternating internal and external rotation maneuvers of the lower limb elongated the nail by $1 \mathrm{~mm}$. The outcomes were assessed clinically and radiographically at a mean of 58.5 months postoperatively.

Results: The gain in femoral length averaged 55,6 mm (range 30$82 \mathrm{~mm}$ ). The healing index was 40 days $/ \mathrm{cm}$. There were 15 excellent results, 1 good and 1 poor. In the 15 excellent results, one patient developed a pseudarthrosis which required further surgery and one presented a failure of the nail which required open femoral osteotomy. The one good and one fair result were 2 deep infections which need to remove the nail and stop the lengthening. In these 2 cases the lengthening obtained was at least $3 \mathrm{~cm}$ less than had been projected. Eight patients underwent ratcheting under general anaesthesia. At the time of follow-up, no patient had axial deviation of the limb secondary to lengthening or knee and hip stiffness.

Conclusions: Femoral lengthening with use of the minimally invasive Albizzia technique provides a reasonable alternative to external fixation that is well tolerated by patients and results in excellent function with little or no distortion of body image.

Significance: The Albizzia nail decrease severe complication rate, which compares with the complications rates of lengthening with an external fixator. But when a complication occurs, this is more severe with high risk of deep infection. 


\section{7}

\section{DELAYED DIAGNOSIS IN SLIPPED CAPITAL FEMORAL EPIPHYSIS (SCFE)}

\section{Bollmann, T. Wirth}

Purpose: Slipped capital femoral epiphysis is one of the most common hip disorders in adolescence. Often enough the diagnosis is not made straight away after first onset of symptoms. This study underlies the patient's career from onset of symptom to final diagnosis.

Methods: From 2000 to 200791 patients were treated for SCFE and included in the study. The patients were classified as follows: stable or unstable slip. The extent of slippage was graded mild $\left(>30^{\circ}\right)$, moderate $\left(30-50^{\circ}\right)$, severe $\left(>50^{\circ}\right)$. We recorded the time interval from the first onset of symptoms to the first medical examination. It was documented if the first medical contact was with a non-orthopedic or orthopedic physician. We also paid attention to the type of symptoms, the patient had initially.

Results: From 91 patients there were 71 with a stable and 20 with an unstable slip. 55 patients had a mild, 26 patients a moderate and 10 patients a severe degree of slippage. All unstable slips were diagnosed within 0.72 weeks from onset of symptoms. 37 patients had a significant delay of diagnosis from the initial medical visit $(40.6 \%)$. Among those were $36 \%$ of all patients with a mild, $35 \%$ of patients with a moderate and $80 \%$ of patients with a severe slip. The delay of diagnosis was 3.3 weeks in patients with a mild slip, 6,5 weeks in cases with moderate slips and 23,6 weeks in those with severe slips. The delay after a visit with a non orthopedic physician was 8.2 weeks at average and 3.68 weeks after initial contact with an orthopedic surgeon. If patients complained of knee pain initially there was a significant delay in diagnosis which was 10.4 versus 4.5 weeks after hip pain complains.

Conclusions: There was a significant delay in diagnosing stable SCFE. The severity of slippage correlates well with the time of delay of diagnosis.

Significance: Initial contact with non-orthopedic physicians results in a higher likelihood of diagnostic delays.

\section{8}

\section{SLIPPED UPPER FEMORAL EPIPHYSIS: A RETROSPECTIVE STUDY OF FIXATION WITH A SINGLE SCREW: THE IMPORTANCE OF CORRECT SCREW PLACEMENT TO OBTAIN GOOD RESULTS}

\section{R. Kanwar, C. E. Bache, P. Glithero, R. Bushan, V. Killampalli, V. Vallamshetla}

Purpose: To evaluate the existing practice of managing SUFE with in-situ pinning using a single cannulated screw.

Methods: 56 children (79 hips) with SUFE were included in this retrospective study. There were 23 bilateral cases. Prophylactic pinning in 15 cases. The slips were graded in severity; 18 preslip, 37 mild, 19 moderate and 9 severe. All hips pinned in situ. Partially threaded screws used in 45 hips, fully threaded screws in 34. Average follow up was 4 years (2-8). Radiologically position of screw in the capital femoral epiphysis and number of threads across physis critically analyzed. The head of femur was classified into three zones on both AP and lateral views; Central, Intermediate and outer. Fixation considered good if the pin was in central zone on both AP and lateral views. The fixation was satisfactory if the pin was in intermediate zone and poor if pin in the outer zone.

Results: Following the above criteria, 27 hips were good, 33 satisfactory and 19 poor. 37 hips had 4 threads across the physis, 27 had 3, 11 had 2 and 4 hips had 5 threads across physis. Overall $88 \%$ had no pain at long term follow up. Slip was controlled in $96 \%$. In three hips screw penetrated joint. In three hips, screws backed out. AVN was encountered in one hip and sub-trochanteric fracture in another. Three patients had revision surgery, six proceed to imhauser osteotomy Central screw placement had predictably good outcome. Screws with less than three threads crossing the epiphysis or in non central positions were associated with complications.

Conclusions: Pin placement is critical for obtaining good results. Ideally a minimum of three threads are needed to cross the physis in both views. This is best achieved with central pin placement on both AP \& lateral views.

Significance: Pin placement is critical for obtaining good results.

\section{9}

\section{COMPLICATIONS AT SCREW REMOVAL IN SLIPPED CAPITAL FEMORAL EPIPHYSIS BY CANNULATED TITANIUM SCREWS}

\section{F. Fernandez, K. Parsch, O. Eberhardt, T. Ilchmann, T. Wirth}

Purpose: Various modes of fixation are proposed for the fixation of slips on the capital femoral epiphysis. We describe our experience with the removal of a new, cannulated titanium screw (Asnis III, Stryker ${ }^{\circledR}$, Howmedica).

Methods: In the 4 years period from 2001 to 2004 single cannulated titanium screws had been inserted in 76 patients (141 hips). These screws were used for in situ fixation of minor chronic slips in 69 patients and for prophylactic fixation on the contralateral side in patients with open physis in 72 patients.

Results: The insertion of these screws was achieved without any problems. When we started to remove the pins the problems began. Screw retrieval was attempted in 51 patients with 97 screws. The average surgical time for removal was double the average time of insertion with $56 \mathrm{~min}(26-240 \mathrm{~min})$. The average interval between insertion and retrieval of screws was 23 months (640 months). All screws are fixed very well. 2 patients sustained a subtrochanteric fracture after screw removal, 16 patients needed extensive chiselling. 11 pins could not be totally removed. $4 \mathrm{~Pa}-$ tients show a lyse around the screw. We did not find statistically correlation between the operating time for screw retrieval and the time interval between insertion and attempted removal or the age at screw retrieval.

Conclusions: Due to the considerable disadvantages encountered in our series about the high morbidity during screw retrieval, we conclude that the complications encountered with the Asnis III screws are unacceptale. Asnis III screws should not be used any more for pinning of slipped capital femoral epiphysis, unless one plans to leave them in for the rest of life.

Significance: Asnis III screws should not be used any more for pinning of slipped capital femoral epiphysis, unless one plans to leave them in for the rest of life. 
140

\section{APPLICATION OF FUNCTIONAL KNEE-SPICA-CAST FOR TREATMENT OF CONGENITAL HIP DISLOCATION WITH CHILDREN OLDER THAN 6 MONTHS}

\section{S. Mikhovich, V. Anosov}

Purpose: Improvement of conservative treatment of DDH. Methods: Femoral head centralization is one of the most important conditions for dysplastic hip joint treatment. Since a child starts to seat Pavlik harness do not provide constant abduction and sufficient centralization of femoral head. A number of devices are introduced for this age group. But the majority of known orthodevices do not provide the desired hip abduction and internal rotation, necessary for the right centralization of the head in acetabulum. Without required hip internal rotation femoral head cannot be centered. According to our method, hip torsion degree is measured with ultrasound. Femoral neck position is studied in two planes. The desired internal rotation angle for treatment in spica-cast is measured with sagittal ultrasound.

Results: For the latest 20 years we have been using a special cast for DDH treatment with children older than 6 mo. Due to this cast the desired and constant abduction and internal rotation are provided. A child can easily sit and walk with the cast. Also this cast allows physiological motion in hip joint, which is very important for treatment. Cast consists of two knee elements connected by a spreader. Fixation time in the cast depends on the age of a child and the severity of dysplasia. The minimum period of fixation is 2 months. During the fixation period a rehabilitation program was conducted (massage, electrical stimulation of gluteal muscles, thermal procedures, electrophoresis, which facilitate strengthening the blood flow of hip joint and optimize bone ossification).

Conclusions: Hip joint development is controlled by ultrasound and X-ray (if necessary). This tactics allows us to obtain good results in majority of the cases.

Significance: This tactic aloud us to increase amount of good treatment results in our clinic.

\section{1}

\section{THE RESULTS OF OPEN AND CLOSED SALTER OSTEOTOMY IN TONNIS TYPE 1 AND TYPE 2 DEVELOPMENTAL DYSPLASIA OF THE HIP}

\section{A. Eren, A. Dost, B. Akman, M. Çakar, M. Guven}

Purpose: We evaluated the results of Salter osteotomy which was performed with or without capsulotomy in patients older than 18 months of age with Tonnis Type 1 and Type 2 Developmental Dysplasia of the Hip (DDH).

Methods: One hundred eighty-nine children older than 18 months of age were operated because of DDH. Preoperative grading of dislocation was based on Tonnis classification. Eighty-six children $(45.5 \%)$ had Tonnis Type 1 and Type 2 dislocations. Twenty-four of them (Group 1) were operated by closed (without capsulotomy) Salter osteotomy, and the remaining 62 (Group 2) were operated by open (with capsulotomy) Salter osteotomy. Nineteen group 1 (18 girls, one boy; 14 Type 1 and 5 Type 2) and 55 group 2 patients (48 girls, 7 boys; 7 Type 1 and 48 Type 2) with at least 2 years follow-up were included in the study. The average age of patients was 25.7 months (range 18-60 months) in group 1 and 24.1 months (range 18-72 months) in group 2. The mean acetabular index angle was $34^{\circ}$ (range $28^{\circ}-42^{\circ}$ ) in group 1 and $39.3^{\circ}$ (range $28^{\circ}-55^{\circ}$ ) in group 2 preoperatively. Arthrography was not used in any patients during the operations. Antero-posterior X-ray views of the pelvis were obtained with $\mathrm{C}$-arm machine after iliopsoas tenotomy. The decision of Salter osteotomy without capsulotomy was made for the following reasons: concentric hip joint reduction in $20^{\circ}$ of abduction; no femoral head lateralization compared with counter lateral side; the absence of superolateral capsular laxity and hip joint instability. The statistical analysis was performed by using "SPSS 14.0 for Windows Evaluation Version" program. The results were analyzed using the Student- $t$ and Chi-square tests. A p value of $<0.05$ was considered to be statistically significant.

Results: The average follow-up time was 2.9 years in group 1 and 2.5 years in group 2 patients (range $2-7$ years). None of the patients in both groups had redislocation. The acetabular index angle in the early postoperative period was $23.5^{\circ}$ (range $20^{\circ}-32^{\circ}$ ) in group 1 and $28.1^{\circ}$ (range $15^{\circ}-42^{\circ}$ ) in group 2 on average $(P>0.05)$. The mean acetabular index angle at the final follow-up decreased to $17.8^{\circ}$ (range $10^{\circ}-26^{\circ}$ ) in group 1 and $20.6^{\circ}$ (range $10^{\circ}$ $\left.32^{\circ}\right)$ in group $2(P>0.005)$. There was not any avascular necrosis of the hip joint in patients operated with closed Salter osteotomy. However, four $(7.2 \%)$ patients which had Tonnis Type 2 DDH in group 2 had avascular necrosis $(P<0.05)$.

Conclusions: Salter innominate osteotomy without capsulotomy can be performed in Tonnis Type 1 and Type 2 DDH. The intraoperative assessments of concentric hip joint reduction, femoral head lateralization and capsular laxity under image intensifier are the key factors for the decision of closed Salter osteotomy.

Significance: The vascular structures on the inferomedial part of the joint capsule can be intact and consequently iatrogenic avascular necrosis can be avoided with closed Salter osteotomy.

\section{2}

\section{ACETABULAR COVERAGE PROCEDURES AFTER CONSERVATIVE TREATMENT OF DDH}

\author{
O. Adamec, J. Chomiak, M. Frydrychova, M. Ostadal, P. Dungl
}

Purpose: To analyze outcomes of conservative treatment of DDH using overhead traction in sequence with coverage procedures of the hip.

Methods: During last 12 years a total 166 patients (197 hips) were treated for hip dislocation using the modified over head traction technique. The average age at the beginning of this treatment was 5.3 months, bilateral dislocation was founded in 31 patients. The final follow-up for re-dislocation was 6 months and for consecutive coverage procedure until 6 years of age. Treatment was performed during 5-6 weeks, after initial horizontal traction we moved to a 4-week over head regimen wherein we increased the abduction by $10^{\circ}$ every 5 days. After reduction verified by arthrography therapy continued with spica plaster cast for 6 weeks follows by Pavlik harness.

Results: From this group of patients described conservative treatment was successful in 168 of $197(85.3 \%)$ hips and closed reduction was permanent. For insufficient acetabular growth and coverage the reconstructive procedures as Salter pelvic osteotomy (27 cases) and Pemberton acetabuloplasty (11 cases) were performed. The time for indications according $\mathrm{AC}$ and $\mathrm{CCE}$ angle ranging from 2.5 
to 6 years of age. The most of described operations were performed on hips grade IV and III B acc. to Graf and in the patients treated after 4 months of age.

Conclusions: The natural acetabular growth is depending on early and tender reduction of dislocated hip in DDH and the after treatment till normal sonographic decision of hip appearance increased necessity of later surgical procedures.

\section{3}

\section{TREATMENT OF DEVELOPMENTAL DYSPLASIA OF THE HIP (DDH) IN CHILDREN BETWEEN THE AGES OF 13 AND 18 MONTHS: A COMPARATIVE STUDY OF CLOSED REDUCTION VERSUS OPEN REDUCTION}

\author{
H. Ghoneem, A. M. El-Sayed
}

Purpose: This study aims at comparing the results of treating two groups of children with DDH aged between 13 and 18 months by closed reduction versus open reduction.

Methods: A series of 40 hips with DDH was prospectively studied. These children were randomly allocated to one of two treatment groups: either closed reduction or hip spica cast (20 hips) or open reduction (20 hips). Both groups were followed up for a mean period of 28 months. We looked at the incidence of redislocation, the incidence of avascular necrosis, radiographic results and clinical results. Radiographic results were classified according to the criteria established by Severin. Clinical results were determined by using a modification of McKay criteria.

Results: Avascular necrosis occurred in 2 out of 20 hips in the open reduction group $(10 \%)$, compared to none in the closed reduction group (the difference was statistically significant). In the closed reduction group, there was one hip with a failed reduction in which subsequent open reduction and pelvic osteotomy was required. In the first year after reduction, the acetabular angle improved rapidly, especially in the younger children. In the open reduction group, there was one hip with redislocation. The treatment time was significantly shortened in the open reduction group.

Conclusions: DDH in children aged between 13 and 18 months can be safely and effectively treated by closed reduction and hip spica cast. Gentle reduction, avoiding extreme abduction, proper immobilization time and adequate compliance of patients significantly improved our results.

Significance: Based on our study, closed reduction and spica cast as a treatment for DDH in children aged 13 and 18 months should be tried by an experienced surgeon in all cases. Open reduction should be done only in those hips that cannot be reduced by closed methods.

\section{4}

\section{ULTRASONOGRAPHIC SCREENING OF DDH IN CLINICALLY SUSPECTED CASES}

O. T. Eren, C. Sever, H. B. Sezer, M. F. Erol, R. Armagan, U. Kuzgun

Purpose: DDH is a silent condition which can be missed easily. Early diagnosis of DDH is essential for early treatment. In this study the use of ultrasonography in clinically suspected cases is evaluated. Methods: 182 babies are bilaterally examined ultrasonographically by Graf's method who were referred from pediatry or obstetrics clinics with clinical suspection of DDH according to first examination and birth feature (pili asmmetry, limited hip abduction, breech presentation, etc.).

Results: Dysplasia is found in 24 babies (\% 13), 5 babies with bilateral involvement. 14 rights, 15 left hip dysplasia detected. Of those 1 was type 4, 4 were type 3 and 22 were type 2 according to Graf's classification. 6 babies treated with Pavlik bandages conservatively.

Conclusions: The use of hip ultrasonography in DDH screening has been subject of debate and confusion but we think that ultrasonography is a good solution to clarify the condition Significance: We highly recommend hip ultrasonography for neonates in suspicious cases with risk factors.

\section{5}

\section{PAIN AND FUNCTION IN CHILDREN WITH AVASCULAR NECROSIS OF THE HIP}

\author{
A. Roposch, A. Hashemi-Nejad, A. Offia, D. Eastwood, \\ J. Hartley, L. Liu
}

Purpose: To determine the relationship between radiographic appearance and clinical severity of AVN of the hip.

Methods: 34 patients who were identified to have radiographic signs of AVN of the hip were assessed at a mean age of 14.4 years (SD 2.1). Radiographic severity of AVN was graded according to Bucholz and Odgen (grades 1-4) by 2 observers in consensus. We hypothesized that, on average, patients with Bucholz grade 4 would have more severe AVN than subjects with Buchholz grade 1 , and that should be reflected in the mean function scores. The CHOHES is a disease-specific measure for hip disorders in children with 3 domains - pain, function, physical exam. It was administered by a single physiotherapist, who demonstrated excellent reliability (ICC $>0.75$ ). The ASK is a self-reported measure of physical function for children. The maximum score for both instruments is 100 and higher scores are favorable.

Results: The mean CHOHES and ASK scores were $82.91(95 \% \mathrm{CI}$ $78.91,86.90)$ and $93.92(95 \%$ CI $91.30,96.54)$, respectively. Radiographically, 8 children had grade 1 (mild) AVN, 6 children had grade 2, and 10 children equally had grades 3 and 4 (severe) AVN. The mean CHOHES scores differed significantly across all 4 radiographic grades $(P=0.004)$. In contrast, the mean ASK scores did not $(P=0.935)$. The mean pain score was $34.41 \pm 6.60$ ("mild to no pain") and it was not significantly different across all 4 Bucholz grades $(P=0.086)$. CHOHES and ASK scores did not correlate $(\mathrm{r}=0.15)$.

Conclusions: Hip function was limited in children with AVN of the proximal femoral epiphysis. Children with more severe radiographic forms of AVN showed less favorable findings. Interestingly, their overall physical function was only mildly limited. Significance: Children with AVN are coping well in terms of their overall physical function despite limitations in their hip function.

\section{6}

MODIFIED TREATMENT STRATEGY OF CONGENITAL DISLOCATION OF THE HIP-IS IT ADVISABLE TO DO AN OPEN REDUCTION IN CDH DURING THE FIRST YEAR OF LIFE, IF CLOSED REDUCTION FAILS?

O. Birke, F. Hefti, R. Brunner 
Purpose: Treatment algorithms in the current literature concerning congenital dislocation of the hip $(\mathrm{CDH} / \mathrm{DDH})$ generally recommend the use of open reduction after failed closed reduction, even in the first year of life. The rates of avascular necrosis (AVN) are higher after open versus closed reduction. The best time of reduction however is discussed with controversy: Is it better to have the benefit of the superior acetabular remodeling, healing and developmental potential during the first few months of life? Or should we wait for the presence of the ossific nucleus within the cartilaginous femoral head? Or should we even wait with an open reduction until it can be safely combined with a pelvic osteotomy? Methods: We conducted a retrospective study with 22 congenital hip dislocations (21 patients) treated with open reduction in our institution from 1996-2005 to analyze the requirement of secondary procedures after early open reduction alone vs. open reduction in combination with a pelvic osteotomy. As of 2006 we no longer performed open reductions in the first year of life. Instead we patiently persisted with closed reduction measures including repeated overhead-extension and abduction of more than $60^{\circ}$ in the hip spica cast if necessary for reduction and retention.

Results: From 1996 to 2005 early open reduction without pelvic osteotomy was performed in 10 hips, $60 \%$ (6/10 hips) required a secondary procedure. In the same period of time only 1 patient $(8 \%$, $1 / 12$ hips) required a secondary procedure after open reduction in combination with a pelvic osteotomy. On the basis of this experience, we modified our treatment strategy: As of 2006 we managed a closed reduction of all dislocated hips in the first year of life, including six hips that had been branded "impossible" elsewhere - these had been planned to undergo open reduction in other pediatric orthopedic centers. Illustrative cases will be presented. Conclusions: In our experience a closed reduction is possible in almost if not all cases of congenital hip dislocation in the first year of life. The benefits of an earliest possible closed reduction outweigh its risks by far. AVN rates as well as clinical and radiologic outcome are better after early closed vs. early open reduction. The reoperation rate after early open reduction alone is unacceptably high. In contrast, there are excellent results after open reduction in combination with a pelvic osteotomy. We therefore wait with an open reduction until it can be safely combined with a pelvic osteotomy at the age of about 1.5 years.

Significance: The study questions a currently widely used treatment practice.

\section{7}

\section{LOSS OF CORRECTION AFTER TRIPLE STEEL OSTEOTOMY FOR ACETABULAR DYSPLASIA IN ADOLESCENTS}

\section{I. Šmigovec, D. Antičević, M. Hašpl, T. Ćuti, T. Đapić}

Purpose: To evaluate radiologic parameters after modified Steel osteotomy performed for developmental dysplasia of the hip in adolescents and young adults.

Methods: Radiographic observations were made preoperatively, immediately after surgery, at 2 years and at final review 4-9 years (mean 6.6) after surgery. Radiographic assessment was by plain anteroposterior radiographs and included: Centre-edge angle of Wiberg, the acetabular angle of Sharp and femoral head coverage. Results: Sharp angle and femoral head coverage improved after surgery and no statistically significant loss of correction was noted during follow-up. The mean Centre-edge angle improved immediately after surgery. Statistically significant (Paired Wilcoxon test $\mathrm{P}=0.0019$ ) decrease in mean CE angle 2 years postoperatively was noted suggesting loss of correction. Further loss of correction was not significant.

Conclusions: We assume that loss of correction 2 years after surgery is due to an autograft resorption and further investigations regarding body weight influence, early full weight bearing and other possible factors that could contribute to loss of correction are needed.

Significance: Loss of correction could be avoided in future by delaying full weight bearing, instructing patients to reduce body weight, encourage them not to gain weight or using a different osteotomy like Ganz or similar in selected cases.

\section{8}

\section{ARE THERE ANY GAIT DEVIATIONS AFTER PERTHES DISEASE?}

\section{B. Westhoff, A. Petermann, R. Krauspe}

Purpose: In Perthes disease the final outcome is dependant on the deformity of the femoral head and the congruity of the hip joint. Till now parameters influencing the severity of head-involvement and the final outcome are poorly understood. Follow-up-evaluations are analyzing only subjective results, clinical parameters like ROM-measurements and radiological changes. There are no studies evaluating the functional impairments during gait.

Methods: 12 children $(8 \hat{\jmath}, 4$, average age $9.7 \pm 3.7$ years. were included. Inclusion criteria were: 1 . unilateral hip involvement in late reparation or final stage 2. Age $>6$ years. 3. No previous surgical treatment at the hips 4 . No other disorder leading to gait deviations. All children were investigated clinically and radiographically. 3D-gait-analysis was performed with a VICON 512 system. Patients walked at a self-selected speed-barefoot. Results were compared to a group of normal children $(n=30,14$ ऽ, 16 우, average age 8.1 years). Sagittal plane deviations were analyzed by the modified "hip flexor index" (HFI) according to Schwartz et al. and frontal plane deviations according to Westhoff et al. The X-rays were categorized according to the Index of Heyman and Herndon. Results: The gait analysis showed in $33.3 \%$ of the patients a normal gait pattern in sagittal and frontal plane. A pathological gait pattern was found in $16.7 \%$ only in sagittal plane, in $16.7 \%$ only in frontal plane and in $33.3 \%$ in sagittal and frontal plane. The radiological evaluation revealed in $25 \%$ excellent, $25 \%$ good, $41.7 \%$ moderate and in $8,3 \%$ bad results. The correlation of the results showed, that all the patients with a normal gait pattern in sagittal and frontal plane had a good or excellent radiological outcome. Three of 4 patients who showed a pathological gait pattern in frontal and sagittal plane had a moderate radiological outcome.

Conclusions: Two thirds of patients after Perthes disease are showing gait deviations despite subjective lack of discomfort. Further studies have to demonstrate whether therapeutical efforts - conservatively or surgically - are able to normalize the movement patterns and to improve long-term prognosis.

Significance: Gait deviations after Perthes disease are correlated with a moderate radiological outcome.

\section{9}

\section{FEMORAL VALGUS OSTEOTOMY FOR ADVANCED FEMORAL HEAD OSTEONECROSIS AND PERTHES DISEASE IN CHILDREN AND ADOLESCENTS}

M. G. Vitale, B. Kaufman, D. P. Roye, H. Matsumoto, J. A. Gomez, J. E. Hyman 
Purpose: The current literature lacks a truly comprehensive examination of the use of a valgus osteotomy to treat osteonecrosis of the femoral head and Perthes disease in the pediatric and adolescent population. Owing to the severity of the pathology, a retrospective examination of the success of valgus osteotomies in treating avascular necrosis and Perthes disease in children and adolescents is warranted.

Methods: Twenty-four patients with diagnosis of osteonecrosis of the femoral head or Perthes disease treated between 1995 and 2007 with a proximal femoral valgus osteotomy were identified. The causes of avascular necrosis were Perthes $(n=14,60.9 \%)$, slipped capital femoral epiphyses $(n=4,17.4 \%)$, femoral neck fracture $(n=2,8.7 \%)$, hip septic arthritis $(n=2,8.7 \%)$ and developmental delays and idiopathic osteonecrosis $(n=1$, $4.3 \%$ ). The average age of receiving a femoral valgus osteotomy was $10 \pm 3.6$ years. The average age at follow-up was $12.2 \pm$ 4 years, mean time of follow-up from the initial valgus osteotomy to last assessment was $23 \pm 15.3$ months.

Results: Postoperative pain improved significantly compared to preoperative pain $(P=0.002)$. Seventy five percent of patients $(n=9)$ who had limitations in their daily activities preoperatively reported that they no longer experienced them after the treatment. External rotation $(P=0.005)$ and abduction $(P=0.003)$ improved significantly at follow up. Postoperative Ficat \& Arlet classification was significantly better when compared to the classification before valgus osteotomy $(P=0.007)$. Univariate analysis showed that sixty percent of patients $(n=6)$ who had Perthes had improved in their Ficat \& Arlet stage whereas one $(14.3 \%)$ patient with a different diagnosis improved $(P=0.082)$. Complications from the surgery were reported in 5 of the $23(21.7 \%)$ cases.

Conclusions: The results of this study suggest that proximal femoral valgus osteotomy is a safe and effective treatment method for osteonecrosis of the femoral head in the pediatric population. Perthes patients improved their femoral head shape more than all other etiologies; there was a trend toward statistical significance in this change. Proximal femoral valgus osteotomy may improve pain and activity limitations in children with osteonecrosis of the femoral head. It is also effective at improving range of motion and femoral head X-ray appearance of the femoral head for these children.

Significance: These findings have leaded the researchers to conclude that a proximal femoral valgus osteotomy is a valid, safe and successful treatment option for a child afflicted with an osteonecrotic femoral head. Specifically for patients with Perthes disease which require the procedure, results demonstrated it's effectiveness over other etiologies.

\section{0}

\section{MID TERM RESULTS OF OPERATIVE AND CONSERVATIVE TREATED LEGG-CALVE-PERTHES DISEASE WITH FOCUS ON QUALITY OF LIFE AND ABILITY TO COMPETE IN SPORTS}

\section{J. Jung, D. Kohn, F. Bachelier}

Purpose: Legg-Calve-Perthes disease is one of the most important diseases in children orthopedic surgery. Little is know about the quality of life and the ability to do sports after Legg-CalvePerthes disease in the mid turn results.

Methods: In this retrospective Study we were able to fully investigate 44 patients with 29 surgically treated and 29 conservative treated hip joints. Only patients with complete X-ray history were included. We performed clinical examination, questionnaire and $\mathrm{X}$-ray control. We obtained data with an average follow up of 6,5 years. We also were able to get full data for Harris Hip Score, Merle D́Aubigne Score, Tegner Activity Score, SF 36 and Stulberg and Harring + Catteral Classification. Plus an own made questionnaire for sports ability.

Results: We were able to show that quality of life and the ability to do sports is significantly reduced even before arthritis causes pain. These results show that not only arthritis on the long term like Stulberg advocated is causing problems. The children suffer long time before arthritis arises.

Conclusions: It is important - especially in cases with bad prognosis to lead parents and children - so children are able to find a job where they can work and to define realistic aims in sports.

\section{1}

\section{LONG TERM FOLLOW UP OF PERTHES DISEASE TREATMENT BY MODIFIED SALTERS OSTETOMY}

\section{K.H. El-Deeb}

Purpose: Salter's innominate osteotomy is originally a redirectional rather than a containment one. Pulling distal pelvic ligament anteriorly and keeping the osteotomy site closed posteriorly is the main philosophy of the procedure. To change this osteotomy to a containment one we applied few modifications. Displacement of the pelvic fragment was anterolateral instead of being anterior, the osteotomy site was opened posteriorly \& the triangular graft was jammed obliquely to keep the anterolateral displacement.

Methods: 80 hips in 76 patients were operated upon. Their ages ranged 3.5-14 years. Their radiological typing according to Catterall classification was as fellows: 46 hips Catterall IV, 29 types III and 5 type II. The cases were followed up for 9-27 years (mean 15.8 years). The results were assessed by a modification of Muller and Seddon point system both clinically and radiologically. At the end of follow up, 71 hips were rated satisfactory and 9 unsatisfactory.

Results: Factors that were found to affect the results were: (1) Age at onset of the disease: the younger the age the better were the results. (2) Radiological type: type II and III Caterall had significantly better results than type IV. (3) Head shape: using Mose template, heads with more than $4 \mathrm{~mm}$. differences in both views had significantly worse results than those having $2 \mathrm{~mm}$ or less. (4) Head cover: as measured by Wiberg angle, hips achieving more than $25^{\circ}$ significantly better results. (5) Neck shaft angle: the mean angle in the satisfactory group was significantly higher than in the an satisfactory.

Conclusions: Modified Salter's innominate osteotomy proved to be a good contribution to the treatment of Perthes disease with $89 \%$ satisfactory results.

\section{2}

\section{MANAGEMENT OF TIBIAL HEMIMELIA}

\section{G. A. Hosny}

Purpose: Congenital deficiency of the tibia or tibial hemimelia is very rare. The incidence in the United States is about 1 per million 
live births (Brown 1971). Ablative procedures are the standard treatment for these cases. An early amputation is accepted by the child as "congenital amputation" (Fernandez Palazzi 1998). Amputation is not an acceptable way of treatment in our community. Therefore the families prefer to leave the affected child without treatment which is a very handicapping condition.This report represents our way of reconstruction of these cases.

Methods: From 1993 till 2005, 23 cases of tibial hemimelia: congenital hypoplasia, type Ia, Ib, II, IV Jone's classification had been treated in our centre. 4 cases of type Ia were bilateral but the operation was performed on one side only in two of them. In 4 cases the knee was fused in flexion elsewhere. All cases were presented to us late as the age of patients ranged from 1.5 years to 29 years. The follow up period ranged from 1 to 8 years with an average of 3 years.

Results: The patients and their families were satisfied in all cases. The four cases type I who were not ambulating before the operation, could walk using knee ankle foot orthosis as there was residual instability of the knee and ankle joints. The 6 cases type II could walk independently and perform normal daily activities without pain. There was $1.5 \& 2.5 \mathrm{~cm}$ shortening in two cases which had been compensated by residual equinus foot deformity. The period of treatment ranged from 12 months to 21 months with an average of 16.5 months. Complication: (1) Pin tract infection (All cases). (2) Cutting through of calcaneal wire ( 2 cases). (3) Knee flexion deformity (5 cases). (4) Fracture of the femoral regenerate ( 1 case). (5) Fracture of the transferred fibula (2 cases). (6) Fracture of the tibial regenerate in one case 7 . Fracture related to the upper half pin of the femoral frame in one case 8. Fracture of the lower femur due to direct trauma during treating a type IV cases not related to the procedure.

Conclusions: Reconstruction of these cases described here is difficult, lengthy and required experience. Amputation is an easy and effective solution but we could not compare it with our results due to the different parameters used in evaluation.

Significance: We believe the method of treating tibial hemimelia described in this series can be justified to reconstruct these cases which refuse amputation as marked functional improvement can be expected.

\section{3}

\section{ROTATION PLASTY WINKELMANN TYPE IIIA FOR PROXIMAL FEMORAL FOCAL DEFICIENCY (PFFD) AND TUMORS WITH ABSENCE OF COXAL FEMORAL END}

\section{G. U. Exner, A. Schchurovski, L. Ramseier, W. Winkelmann, Y. Reiland}

Purpose: Rotation plasty with fixation of the distal femur to the pelvis replacing the hip joint with the knee joint has been used to treat PFFD with severely deficient coxal end of the femur. Because of the frequently associated deficiency of the anterior cruciate ligament and hypoplasia of the lateral femoral condyle the knee joint frequently fails at long term. The lack of rotation is also an important draw back. Winkelmann has therefore introduced a technique to position the rotated tibia into the hip joint. Initially this was used in tumor cases, when complete removal of the femur is mandatory and applied this also for PFFD. We want to communicate 3 cases, where we have applied this technique in a child with PFFD or tumor problem.

Methods: (1) PFFD: This boy was followed since birth. The femur was unstable throughout. At the age of 6 years only the femoral head anlage and the distal femoral stump were present in imaging studies. Needing a pelvic support for the orthoprosthesis made hygiene difficult for the child. From the small bony structures developed it appeared impossible to achieve a stable fusion between the femoral head and the distal femoral structures. It was chosen to remove the femoral remnants and to perform a rotation plasty positioning the lateral tibial plateau into the hip joint; pelvifemoral muscles were transposed. (2) PFFD: 6 year old girl-similar situation as \#1. (3) Osteosarcoma of the femur, 6 year old boy.

Results: At 1-3 year $\mathrm{f} / \mathrm{u}$ the patients use an orthoprosthesis as used for rotation plasty without pelvic support. For toilet use the prosthesis needs not be removed. They follow same active as his peers.

Conclusions: The functional results in the cases presented suggest, that this technique may be considered in severe femoral deficiency. The question however must be raised if this is possible only when a femoral head is present indicating a sufficiently well developed acetabulum.

Significance: In our observations this type of rotation plasty is superior to fusion of the rotated distal femur to the acetabulum as the new procedure allows for rotation which is of high importance for the hip joint.

\section{4}

\section{DEFORMITY CORRECTION WITH AXIAL LENGTHENING AND PHYSIODESIS AFTER PARTIAL PHYSEAL ARREST}

\section{J. Horn, H. Steen, L. Kristiansen}

Purpose: Partial growth arrest after physeal injury might cause angular deformity and leg-length discrepancy (LLD). When spontaneous correction after physeal bridge resection is unlikely and the deformity is symptomatic with a risk for secondary structural changes in the adjacent joints, an acute or gradual correction before skeletal maturity might be required. In this regard complete physiodesis is necessary to prevent recurrent deformity after correction. The aim of our study was to present and evaluate our treatment protocol including: complete physiodesis of an injured physis, angular correction and lengthening with overcorrection of length according to loss of remaining growth of the affected physis.

Methods: From February 2001 until September 2007 eight patients were operated at our institution with angular correction and lengthening in combination with complete percutaneous physiodesis after partial physeal arrest. Five patients ( 3 males, 2 females, age 12-14 years) were followed until skeletal maturity. Lengthening was done with either an Ilizarov apparatus or with a Taylor Spatial Frame. Three patients had a partial physeal arrest in the distal femoral physis, 2 in the proximal tibial physis. All patients had severe angular deformities of $12^{\circ}-24^{\circ}$ and LLD's of 15 $60 \mathrm{~mm}$. LLD at maturity was estimated based on Green-Andersons growth chart. Mean overcorrection of length according to remaining growth of the affected physis was $12 \mathrm{~mm}(7-15 \mathrm{~mm})$. Results: All deformity parameters were fully corrected in all patients and the frames were removed after bony consolidation. The patients were followed until skeletal maturity. Mechanical axis deviation (MAD) was within $\pm 5 \mathrm{~mm}$ compared to the healthy side in 4 patients, One patient was slightly overcorrected from valgus to varus, resulting in a MAD of $20 \mathrm{~mm}$. Mean LLD at maturity was $6,4 \mathrm{~mm}(3-9 \mathrm{~mm})$, hence LLD at maturity in all our patients was $<1 \mathrm{~cm}$. 
Conclusions: In cases of partial physeal arrest with severe symptomatic deformities, complete physiodesis of the injured physis, angular correction and lengthening with the Ilizarov method with overcorrection of length according to loss of remaining growth of the affected physis is a safe method. All deformity parameters can be fully corrected and LLD at maturity can be provided by overcorrection according to length.

\section{5}

\section{PERCUTANEOUS HEMIEPIPHYSIODESIS USING TRANSPHYSEAL SCREWS FOR ANGULAR DEFORMITIES AT THE KNEE}

\author{
C. Santili, B. G. Schröder e Souza, G. Waisberg, M. Akkari, \\ S. R. Braga, W. Lino Jr
}

Purpose: Asses the clinical and radiographic results of percutaneous hemiepiphysiodesis using transphyseal screws for angular deformities at the knee.

Methods: Twenty-nine patients have been evaluated prospectively, corresponding to 49 knees, of which 35 were valgus and 14 varus. Average age at surgery was 12 years and 10 months and average follow-up time was 4 years and 3 months. In 28 patients the knee physis was closed at the last follow-up. The amount of correction, speed of correction, symmetry of the knee and complications has been analyzed.

Results: Average angular correction until screws removal was $11.80^{\circ}$, which happened 12.08 months after surgery on average. The mean speed of correction was $1.26^{\circ} /$ month, within a large range $\left(0-4^{\circ}\right.$ per month). There has been significant improvement in symmetry of the knees $(P=0,005)$. The mean loss of correction after screw removal was $0.55^{\circ}$. Occurrences had happened in $22.4 \%$ of the knees treated. No bone bridges have been observed post-operatively. Excellent and good results have been achieved in $81.64 \%$ of the cases.

Conclusions: This technique proved to be an easy, quick, low morbidity and efficient procedure, once the principles are respected concerning indications and close follow up.

\section{6 \\ EFFECTS OF BISPHOSPHONATES ON THE COLLAGEN NETWORK IN A RABBIT MODEL OF DISTRACTION OSTEOGENESIS}

\section{Wassen, R. Bank, R. Castelein, R. Sakkers, W. Dhert}

Purpose: Bisphosphonates are used as a successful treatment in children with systemic conditions such as osteogenesis imperfecta. The majority of studies on bisphosphonates, attribute the increase in bone quality after treatment with bisphosphonates to the inhibitory effect on bone resorption. However, information regarding the effects of bisphosphonates on bone formation is hardly available. Since bone quality is a reflection of the collective components of bone mass and the architecture of the collagen network, the collagen synthesis during bone formation plays an important factor in bone strength. The purpose of this study is to examine the effects of bisphosphonates on the collagen network during bone formation.

Methods: In this study we used a rabbit model of distraction osteogenesis to investigate the effects of the bisphosphonates
EHDP and APD on the post-translational modifications of type I collagen and the formation of collagen cross-links during osteogenesis. Cortical callus formation, a contra-lateral cortical bone segment, and cortical bone proximal to the callus were analyzed for their collagen content, lysyl hydroxylation of the triple helix and cross-link pattern, at various time points of distraction osteogenesis. Twenty-four New Zealand White rabbits underwent distraction osteogenesis of the right tibia by the Ilizarov procedure. The rabbits were randomized in three groups containing 8 controls, 8 rabbits treated with EHDP and 8 rabbits treated with APD.

Results: We found a significant increase in the amount hydroxylysines per collagen molecule after 10 weeks in the callus as well as in the cortical bone in the rabbits treated with both the bisphosphonates compared to the control group. The amount of the crosslink LP was increased in the treated groups after 10 weeks, however the total amount of the crosslinks HP and LP was not significantly different. Between the different groups no significant differences were found in the amount of collagen molecules per amount of bone and the ratio between collagenous and non-collagenaous proteins.

Conclusions: The increased bone strength after treatment with bisphosphonates can not only be attributed to the inhibitory effect on osteoclasts but also to a stimulatory effect on osteoblasts. In this study the effects of bisphosphonates on the collagen network in invivo bone are described for the first time. The data indicate that bisphosphonates influences collagen synthesis by the osteoblast. To see whether bone quality is improved by these changes in the collagen network, further research is necessary.

Significance: Since bisphosphonates increase bone strength not only by an inhibitory effect on osteoclasts but also by a stimulatory effect on osteoblasts, they can also affect the collagen synthesis by the osteoblasts. The effects of bisphosphonates on the collagen synthesis might contribute to the increase of bone strength.

\section{7}

\section{ILIZAROV FOOT CORRECTION: EFFECT ON FUNCTION AND JOINT MOTION}

\section{H. A. Kotb}

Purpose: Ilizarov Method is a known tool in the correction of complex feet. functional out come and mobility of the ankle and subtalar joints has been a concern. We report results of 9 years Ilizarov frame feet correction.

Methods: Ilizarov frame was used to correct 43 deformed feet in 32 patients. 22 were males. Mean age at operation was $10.7+6.2$ years. The pre-operative diagnosis was; 17 relapsed idiopathic congenital talipes equino varus, 4 neglected idiopathic congenital talipes equino varus. 10 paralytic feet. 6 rocker bottom feet. 6 talipes equino varus associated with syndromes. 31 feet had previous operations average 1.8 operations. Single tibial ring fixation was used in 37 cases. 3 cases were treated by distraction osteotomies and 40 by soft tissue distraction. 9 cases had additional surgical procedure. Clinical, functional and radiographic evaluation was performed. Results were graded according to Ognesyan et al., Classification. Functionally; mobility, walking distance, one leg stance and hopping were evaluated.

Results: Mean follow up was $62+31$ months. The mean fixation time was $2.7+0.8$ months. All feet were initially corrected except one. At last follow up $31(72.1 \%)$ had good results, $7(16.3 \%)$ had satisfactory results and $5(11.6 \%)$ had unsatisfactory results. There 
has been a significant increase in mobility, walking distance, one leg stance and hopping A significant increase in ankle range of motion $(P=0.026)$ and mean increase in subtalar range of motion $(P=0.22)$ but was not significant. The most common complication was pin track infection, there were no major complications, and no complications related to single tibial ring fixation.

Conclusions: Ilizarov fixator is an effective tool for correction of deformed feet; one tibial ring fixation is adequate to achieve correction. An increase in function and range of motion can be expected.

Significance: A significant increase in function and ankle joint motion can be anticipated with Ilizarov foot correction.

\section{8}

\section{PEMBERTON-LINE OSTEOTOMY OF THE DISTAL TIBIA IN FIBULAR HEMIMELIA TO STABILIZE THE HINDFOOT}

\section{G. U. Exner, L. Ramseier}

Purpose: Fibular hemimelia is associated with a deformity of the distal tibial epiphysis resulting in a convexity with lateral and posterior slope of the distal joint surface with postero-lateral migration or dislocation of the foot. A new technique to shape the distal tibial epiphysis was described by the author (JPO-B 12:27$32,2003)$ with short term results. The patients $(n=3)$ with a f/u more than 6 years are presented.

Methods: 3 patients (4 feet) with longitudinal deficiencies and absence of the fibula have undergone the procedure at ages 7, 13, 18 and 22 months as described in the original article. The procedure consists in extensive soft tissue release, an osteotomy through the postero-lateral metaphysic onto the physis in order to bend the physis down postero-laterally. The reduction held with a bone block taken from the diaphysis and a $\mathrm{K}$-wire.

Results: At f/u at ages 7-11 years 6-9 years after the procedure all feet have been retained underneath the tibia with joint mobility. In 2 patients lenghthenings of the femur and tibia have been performed lateron for correction of the associated PFFDs without dislocation of the reduced ankle joint, in one patient (bilateral case) one tibia was lengthened.

Conclusions: The procedure appears to be an option for stable retention of the feet in fibular hemimelia. Data are too few to recommend an optimum age. It is felt, that appearance of the distal tibial ossific nucleus would be a good time as this allows easier identification for the osteotomy and still presenting enough flexibility of the epiphysis to adapt.

Significance: In our patients this procedure has replaced other reconstruction (e.g., GRUCA-BARDENHEUER, lateral support with a pelvic crest transplant, ankle fusion).

\section{9}

\section{FLEXIBLE FLATFOOT WITH SHORTENED GASTROCNEMIUS MUSCLE-RESULTS OF OPERATIVE TREATMENT}

\section{Lawniczak, M. Napiontek, M. I. Shadi}

Purpose: The purpose of the study was to evaluate combined operative technique used in treatment of the flexible flatfeet in children-opening wedge osteotomy of the anterior part of the calcaneus, tibialis anterior split transfer with tendosuspension and gastrocnemius lengthening. This technique is used in our institution since 1993.

Methods: Pre-op and follow-up both physical and weight bearing radiographic examination were performed.

Results: Final clinical evaluation disclosed longitudinal arch of the foot in standing position restored completely in 12 feet and slight lowering in 2 feet. Push-off power was normal. The parents and patients were satisfied with the shape and function of the feet. On A-P radiographs mean talo-calcaneal angle decreased from $31^{\circ}$ to $22^{\circ}(P=0,002)$. The mean talo-metatarsal 1 st angle was corrected from $165^{\circ}$ to $181^{\circ}(P<0,001)$. On lateral radiographs mean talo-metatarsal 1st angle changed from $160^{\circ}$ to $180^{\circ}(P<0,001)$. On A-P radiographs improvement of the talo-navicular and calcaneo-cuboid displacement was achieved in all feet. On lateral radiographs dorsal displacement of 1 st cuneiform was achieved in all feet. Radiographic overcorrection was observed in both feet of 1 patient, however clinically observed foot arch tended to be high in patients with follow up of 11years (statistical significance according to Wilcoxon test).

Conclusions: This combined surgical procedure is safe and valuable; however, in some part of cases may cause mild overcorrection in long time follow up.

Significance: Opening wedge osteotomy of the anterior part of the calcaneus, tibialis anterior split transfer with tendosuspension and gastrocnemius lengthening can be used in children older than 6 years with flexible flatfoot deformity associated with shortened gastrocnemius muscle, which complain for shoes discomfort and pain.

\section{0}

\section{NON-OPERATIVELY TREATED CLUBFOOT OUTCOMES: DO EARLY RESULTS DETERIORATE OVER TIME?}

\author{
B. S. Richards, L. A. Karol, S. Faulks
}

Purpose: Utilization of the Ponseti casting and French physiotherapy methods have proven effective in reducing the need for extensive surgical release. The purpose of this study was to determine if early results deteriorate over time.

Methods: In May 2003, 101 idiopathic clubfeet (67 patients) treated non-operatively were evaluated with a minimum 12 months follow-up. 59 feet (39 patients) were treated with the Ponseti casting method; 42 feet ( 28 patients) were treated with the French physiotherapy method. We monitored this same group of patients each year to determine if results changed with longer follow-up. Outcomes were classified as good (plantigrade \pm TAL), fair (limited posterior release, ATT or lateral column shortening), poor (complete PMR), or indeterminate (may later require posterior release).

\begin{tabular}{lllll}
\hline Overall results & Good & Fair & Poor & Indeterminate \\
\hline $2003>12$ month $77(76 \%)$ & $5(5 \%)$ & $10(10 \%)$ & $9(9 \%)$ \\
$\quad$ follow up & & & & \\
$2004>24$ month $65(64 \%)$ & $9(9 \%)$ & $14(14 \%)$ & $13(13 \%)$ \\
$\quad$ follow up & & & & \\
$2005>36$ month $65(64 \%)$ & $11(11 \%)$ & $18(18 \%)$ & $7(7 \%)$ \\
follow up & & & & \\
$2006>48$ month $68(67 \%)$ & $13(13 \%)$ & $20(20 \%)$ & N/A \\
follow up
\end{tabular}


Results: Follow-up averaged 58 months (range 50-66). Over time, there was a trend toward less favorable outcomes but this did not reach statistical significance for the entire group or for the Ponseti subgroup itself. The physiotherapy subgroup worsened slightly over time $(P=0.01)$.

Conclusions: Over 4 years of follow-up, outcomes of clubfoot patients treated non-operatively showed a trend toward worsening, evidenced by fewer good outcomes and greater poor outcomes. With the sample size studied, this did not reach statistical significance.

Significance: Reports on clubfoot treatment using 1- or 2-year follow-ups should be interpreted cautiously. Favorable outcomes may not be maintained over time. Recognition of why this occurs is important in order to improve future treatment options.

\section{1}

\section{TALECTOMY FOR RIGID CLUBFOOT DEFORMITY}

\section{H. Elsherbini}

Purpose: to evaluate the effect of talectomy in the treatment of rigid club foot deformity secondary to arthrogryposis multiplex congenita.

Methods: Seven cases (10 feet) have rigid club feet were treated by talectomy in the period between 2001 and 2007. All cases had previous failed soft tissue release .There were 5 males and 2 females. There ages ranged from 2.5 to 10 years with averages 6.2 years. All cases were grade IV according to Dimeglo classification for the degree of rigidity.Three feet need soft tissue release at the same time of talectomy. Navicular was excised at the same time of talectomy in five feet to allow good position of the calcaneus in the ankle mortise. Calcaneus osteotomy with lateral based wedge was carried out in four feet to correct adduction forefoot at the same time of talectomy. The follow up ranges from 6 to 60 months with average 34.5 months.

Results: 9 feet were plantigrade and one foot had residual equinus deformity $15^{*}$ due to incomplete removal of the talus. All cases have painless walking but had marked hindfoot stiffness. All cases become grade II according to Dimeglo classification.

Conclusions: Talectomy is an effective procedure for correction of hindfoot deformity in rigid clubfoot with grade IV Dimeglo classification, provided that talus is completely removed and calcaneus is positioned correctly in ankle mortise.

Significance: Severe rigid clubfoot secondary to arthrogryposis multiplex congenita can be corrected by one stage relatively easy operation with least morbidity.

\section{2}

\section{A NEW APPROACH TO THE SURGICAL TREATMENT OF CLUBFOOT: INDIRECT TALONAVICULAR JOINT REDUCTION}

\section{A. Dogan, G. Zorer, M. Yalcinkaya}

Purpose: Evaluation of the short term results of surgically treated clubfoot cases performed by without opening the talonavicular and calcaneocuboid joint capsules and trapezoid shaped wedge resection of the cuboid bone, therefore reducing the risk of developing avascular necrosis of the talus and navicular.

Methods: 17 feet of 13 patients were operated on by the complete subtalar release technique except talonavicular and calcaneocu- boid joint releases. Mean age at surgery was 13 months (11-17) and mean postoperative follow up period was 17 months (6-25). In all patients the Cincinnati incision was used and tendo calcaneus and tibialis posterior tendon were lengthened by Z-plasty and distal portion of the tibialis posterior tendon was released all the way to navicular insertion. Posterior talofibular and calcaneofibular ligaments were released. Tibiotalar and subtalar joint capsules were released. Talonavicular and calcaneocuboid joint capsules were not opened in any of the patients. After soft tissue release, hindfoot was corrected and fixed by two Kirschner wires and if the lateral border of the foot still looked convex and/or the forefoot was supinated in relation to hindfoot than a trapezoid shaped wedge resection from the cuboid bone was performed and adduction and supination of the forefoot were corrected and the osteotomy site at the cuboid bone was closed and fixed by a Kirschner wire. All patients were evaluated both clinically and radiographically.

Results: AP talocalcaneal angle was $20.2^{\circ}(16-28)$ preoperatively and $34.5^{\circ}(24-41)$ postoperatively. AP talo-1.metatarsal angle was $28.7^{\circ}(19-43)$ preoperatively and $2.8^{\circ}([-6]-8)$ postoperatively. Lateral talocalcaneal angle was $15.5^{\circ}$ (7-22) preoperatively and $28.5^{\circ}(20-43)$ postoperatively. Lateral talo-1.metatarsal angle was $23.2^{\circ}(17-34)$ preoperatively and $4.5^{\circ}([-5]-12)$ postoperatively. Correction in all parameters were statistically significant $(P<0.05)$. Clinically $9(\% 53)$ feet were excellent, $6(\% 35)$ feet were good, $2(\% 12)$ feet were fair. Fair results were related to hindfoot varus. There was no problem in the forefoot alignment either clinically or radiographically.

Conclusions: According to the short term results of this technique, adduction and supination deformity, which is the most common residual deformity after clubfoot surgery, seems to be eliminated. To evaluate the talonavicular reduction definitely, ossification of the navicular must to be waited. Also, the risk of developing avascular necrosis of the talus and navicular should decrease theoretically with this technique.

Significance: According to the short term results of this technique, adduction and supination deformity, which is the most common residual deformity after clubfoot surgery, seems to be eliminated.

\section{3}

\section{BOTULINUM A TOXIN FOR MANAGING FOREFOOT ADDUCTION AFTER CLUBFOOT TREATMENT BY PONSETI METHOD}

\section{Vasilescu, D. Cosma}

Purpose: According to Ponseti method, relapses in clubfoot are treated by re-casting or tibialis anterior tendon transfer. In our series, we observed some cases of relapses with only forefoot adduction. We hypothesized that using the Botulinum A Toxin (BTX-A) in the abductor hallucis muscle could attenuate its function, improving the aspect and function of the foot.

Methods: Fifteen patients with 23 idiopathic clubfeet were recruited. All clubfeet were treated according to Ponseti method before the age of 9 months. At the age of 18 months, we noted the increase of curvature of the lateral border of the foot score (Pirani classification), all other scores being nil. At the age of 2 years we injected 10 UI BTX-A $/ \mathrm{kg}$ in the middle third of the medial border of the foot (abductor hallucis muscle). After injection, all patients were allowed to carry on with their usual activities. We evaluated the Pirani score (with special attention to curvature of the lateral border) before injection, at 2 weeks after injection and at 3 months. Clinical photographs obtained included the following 
views: plantar and medial in weight-bearing. The minimum follow-up period was 1 year for all patients. Any adverse events related to the BTX-A injection were noted.

Results: The mean Pirani score before injection was 0.9. In all cases, the increase of the Pirani score was only due to the curvature of the lateral border of the foot. At 2 weeks after the injection, the mean Pirani score was $0.6(P<0.05)$. At 3 months follow-up, continuing the use of foot abduction brace during the night, the mean Pirani score decreased to $0.4(P<0.05)$. No patients who received BTX-A experienced adverse reactions, side effects, or complications.

Conclusions: In our experience, the most common aspect in clubfoot relapses is forefoot adduction. Some children will require anterior tibialis tendon transfer, typically between ages 2 and 4 years. Before the age of 2 years, re-casting is indicated to correct muscle imbalance of the foot. If the muscle imbalance is due to the abductor halucis contracture, BTX-A injection can safely attenuate its function with improving both aspect and function of the foot. The use of BTX-A as an adjunctive therapy is an effective and safe alternative and one that may be preferable to parents.

\section{4}

\section{THE EXERCISE PERFORMANCE IN CONGENITAL CLUBFOOT PATIENTS AT SCHOOL AGE}

T. Kenmoku, M. Kamegaya, M. Takazawa, T. Saisu, Y. Shinada, Y. Shinohara

Purpose: There is no previous study reported the exercise performance in clubfoot patients objectively. The purpose of this study was to evaluate the exercise performance of patients with congenital clubfoot objectively through their records of physical test at elementary school, and evaluate the correction of foot deformity with radiographic measurements.

Methods: 48 clubfeet of 30 patients who belonged to 1st grade through 6th in elementary school were subjected for this study. There were 18 boys and 12 girls. 9 patients were treated conservatively, 8 patients underwent percutaneous Achilles tendon tenotomy (not Ponseti's method) and 13 patients were treated with extensive soft-tissue release. The average age at this investigation was 8.8 years. All patients could walk normally, and do toe-walk and heel-walk. We collected their records of physical test which was nationwide performed in elementary school. The test consisted of the following 5 events: 50 meter race, standing long jump, repeated side steps test, shuttle run and softball arm throw. We evaluated performance exercise of clubfoot patients to calculated deviation value of each record based on the numerical value that The Ministry of Education announced. With using them, we categorized 2 groups based on deviation value (the group with average value or more, and less than average). In each event, we compared these 2 groups in terms of treatment methods, the amount of ankle dorsiflexion and uni- or bi-lateral clubfeet. To evaluate the correction of foot deformity, we measured 6 angles (The talo-calcaneal angle, the calcaneal $/ 5$ th metatarsal angle, the navicular/1st metatarsal angle on the antero-posterior radiograph, and the talo-calcaneal angle, the calcaneal/1st metatarsal angle, the $1 \mathrm{st} / 5$ th metatarsal angle on the lateral radiograph), And we evaluated the correlation between records of physical test and radiographic angles.

Results: We totally collected 148 individual records from 30 patients. Of which, $144(97.3 \%)$ were within normal range in the 5 events. The average deviation values of all grades were 46.6 in 50 meter run, 48.3 in standing long jump, and 52.5 in repeated side steps, 48.0 in shuttle run, and 51.0 in softball arm throw. On treatment method, the amount of ankle dorsiflexion and uni- or bi-lateral clubfoot, there was no significant difference between the two groups (Mann-Whitney's $U$ test, $P>0.05$ ). On the radiograph, there were the correlation between the calcaneal $/ 5$ th metatarsal angle and the following 3 events: repeated side steps, shuttle run and long jump, and the lateral talo-calcaneal angle and 50 meter run (Spearman's correlation coefficient by rank, $P<0.05)$.

Conclusions: The clubfoot, even surgically treated foot, does not affect on the development of exercise performance. The improvement of metatarsasus adductus influences exercise significantly.

Significance: Clubfoot does not affect on the development of exercise performance at school age.

\section{5}

\section{RESULTS OF CONSERVATIVE TREATMENT OF CONGENITAL IDIOPATHIC CLUBFOOT (FUNCTIONAL METHOD)}

\section{Rampal, P. Wicart, R. Seringe, X. Barthes}

Purpose: Many different conservative ways of treatment of congenital idiopathic clubfoot have been described. This study aimed at giving the results of the functional method.

Methods: The inclusions criteria were as follow: children treated for an idiopathic clubfoot between 1988 and 1990, and before the age 8 months. Minimal follow-up was 8 years. In a continuous series of 80 patients, $21(26 \%)$ were excluded for a lack of followup. The series is about 59 children ( 83 feet). The initial severity of the deformation was classified using Seringe score, and result at last follow-up evaluated using the Ghanem and Seringe one. The treatment was made of passive and active mobilization with a physiotherapist, and splints.

Results: Average age at beginning of treatment was 21.9 days ( 0 days to 8 months 22 days). Mean age at last evaluation was 14 years (7.6-19.2). The serverity at first report was low, average or severe in respectively 18,29 and $43 \%$ of cases. Thirty eight feet $(45 \%)$ were operated on at mean age 3.5 years old. The surgery was an extensive liberation of soft tissues in 7 feet $(15 \%)$ and a more limited one in the others cases. A second surgery was needed in two cases ( $5 \%$ of operated on feet).At last follow-up, the result was "excellent" for 71 feet $(85.5 \%)$, "good" for $10(12 \%)$ and "medium" for $2(2.4 \%)$. A triple arthrodesis was needed once. A severe deformation at birth was correlated with a lower result at final review, and at the frequency of surgery. On the whole series, results were lower if surgery was needed.

Conclusions: Regarding these results, we concluded that functional treatment of clubfoot seems to give good results, with no need of surgery. A better definition of the indications of percutaneous Achilles tenotomy should make the surgical need lower. Surgery gives lower results, specially in term of function. Except for the most severe feet, it seems that good compliance and good quality of the functional treatment are the main prognosis component of good results.

Significance: In conclusion, functional conservative treatment of congenital idiopathic clubfoot seems to be a very efficient way of treatment, with a low complication rate and good functional results. 


\section{6}

\section{INTRAMEDULLARY NAILING IN PAEDIATRIC FOREARM FRACTURES: SINGLE OR BOTH-BONE FIXATION?}

\author{
B. Valentinov, P. Chobanov, P. Tivchev, T. Todorov
}

Purpose: Intramedullary fixation is a well-established method for stabilization of the shaft forearm fractures in childhood. During the last years some authors report for successful results with fixation of one bone only. The opinion in the literature regarding one-side or both-side nailing, still remains controversial. Purpose of the study is to compare and to analyze the outcomes of single and both-bone fixation in the treatment of the pediatric forearm fractures.

Methods: For the period 1997-2006, the flexible intramedullary nailing has been implemented in 78 diaphyseal forearm fractures. The average age was 10.8 years; male/female ratio-3:1. The patients were split into two groups: 25 cases, treated with single-bone fixation (SBF) of the more unstable injury (16 ulna and 9 radius), and, 53 cases with both-bone fixation (BBF). Radiological results and clinical outcomes after Price (1990) have been compared. The statistical testing was performed by means of Student's t-test and Fisher's exact test $-\mathrm{p}$ values $<0.05$ were considered to be significant.

Results: Loss of reduction has been observed in 4 cases which have been treated with SBF. Repeated manipulation was necessary in 2 of them. The other 2 patients were healed with residual angulation $-15^{\circ}$ and $18^{\circ}$. Angulations over $10^{\circ}$ were not observed in the group of BBF. The difference between the two groups was significant $(P=0,001)$. The distribution of the functional outcomes in SBF and in BBF was completely identical. Statistically reliable difference was not found.

Conclusions: SBF really minimizes surgical time and exposure. Actually it is applicable in however more stable shaft injuries but still it does not exclude the risk of a secondary angulations and possible repeated manipulations. It requires frequent X-ray checks as well as comparatively longer immobilization. BBF is a more reliable method of stabilization with all its advantages proceeding from that.

Significance: SBF may be successful only with a careful fracture pattern selection. BBF completely corresponds to the modern requirements for primary definitive fracture care.

\section{7}

\section{POSTERIOR APPROACH TO THE ELBOW BASED ON THE VASCULAR ANATOMY OF TRICEPS BRACHII IN THE TREATMENT OF DISPLACED SUPRACONDYLAR FRACTURES OF HUMERUS IN CHILDREN}

\section{A. S. Rajeev, G. Tawari, J. Pooley}

Purpose: Displaced supracondylar fracture presents a challenge for orthopedic surgeons to obtain an anatomical reduction and good rigid fixation. We have found difficulties performing open reduction using the described surgical approaches. It may not be possible to obtain anatomical reduction of displaced supracondylar fractures in children by closed manipulation. We report an approach based on studies of the vascular anatomy of triceps, which provides a wide exposure facilitating surgery.

Methods: Between 2002 and 2004 we performed open reduction and internal fixation on 12 children ( 8 girls, 4 boys: mean age 6 ).
Our vascular injection studies indicate that the blood supply to triceps brachii is proximally based. The major proximal supply to triceps was provided by the profunda brachii,recurrent branches were seen to arise from the radial and ulnar arteries.It also demonstrated that the blood supply of the triceps muscle is essentially proximal from the branches of profunda brachii. More over the anastomosis between the branches of profunda brachii and radial and ulnar collateral arteries takes place at the fine vessel level and are functionally end arteries. We used a posterior approach identifying the ulnar nerve. We mobilised lateral triceps and anconeus in continuity preserving the vascularity and separated the components of distal triceps through an intermuscular septum. The fractures were reduced and fixed using $\mathrm{K}$ wires.

Results: The fractures healed in the anatomical position in each child and all 12 demonstrated a full range of elbow movements within 6-8 weeks of $\mathrm{K}$ wire removal. We observed no complications.

Conclusions: A surgical approach based on the vascular anatomy of triceps can be used to provide a wide, symmetrical and safe exposure facilitating open reduction and internal fixation of supracondylar fractures of the humerus in children whilst avoiding complications including residual elbow stiffness.

Significance: Although closed reduction and percutaneous K wire fixation remains the treatment of choice for displaced supracondylar humeral fractures, anatomical reduction must be achieved ideally and residual rotation of the fracture fragments avoided. We have found that this surgical approach has reduced our reluctance to proceed to surgical treatment of these difficult fractures and consequently a tendency to accept sub optimal reduction.

\section{8}

\section{ELASTIC STABLE INTRAMEDULLARY NAILING OF THE SEVERELY DISPLACED PROXIMAL HUMERUS AND HUMERUSSHAFT FRACTURES IN CHILDREN}

\section{F. Fernandez, O. Eberhartd, T. Wirth}

Purpose: This study analyzes the results of elastic stable intramedullary nailing of the severely displaced proximal humerus and humerusshaft fractures in children and possible complications of the method.

Methods: We report our experience of elastic stable intramedullary nailing (ESIN) of severely displaced proximal humeral fracture Type III and IV according to Neer and humerus shaft fractures in children. 65 children (average age 12.9 years) with 19 Salter-Harris Type II epiphyseal fractures and 16 children with metaphyseal fractures of the proximal humerus and humerus shaft fracture (transverse: $n=13$, oblique: $n=11$, wedge: $n=3$ ) were treated with ESIN by one-nail or two-nail-technique.

Results: For follow-up examinations after 28 months the children were classified by use of the Constant-Murley-score. In average they gained 97 points (94-100). All children could resume their sporting activities like before. There were the following complications: Two perforations of the nail at the head of the humerus with loss of position, one loss of position without nail perforation, one misplacement of a nail, one revision due to haematoma and two difficult removals of metals, one lesion of the radial nerve (the neurologic lesion healed spontaneously), one superficial wound infection, four Bursitis. Serious complications such as osteitis, pseudarthrosis, and functional disorders were not found.

Conclusions: For the treatment of displaced proximal humeral and shaft humeral fractures in children more than 10 years old the retrograde ESIN represents a safe minimal invasive surgical 
procedure with a high patient comfort. The surgical technique is simple to learn.

Significance: Elastic stable intramedullary nailing is the standard operation to treat severely displaced proximal and shaft fractures in children older than 10 years.

\section{9}

\section{CLOSED AND OPEN REDUCTION (WITH POSTERIOR APPROACH) OF POSTEROMEDIAL DISPLACED GARTLAND TYPE III SUPRACONDYLAR HUMERUS FRACTURES IN PEDIATRIC PATIENTS}

\author{
C. N. Aktekin, A. Toprak, A. M. Ozturk, A. Y. Tabak, \\ B. Ozkurt, M. Altay
}

Purpose: The purpose of our study was to determine if open reduction of displaced supracondylar humerus fractures increases the incidence of complications and have worse outcomes or not. Methods: Eighty-three patients with Gartland Type III fractures treated with either open reduction (through posterior midline incision) or closed reduction and percutaneous fixation were analyzed. 55 patients included to study with a mean follow-up of 22 months (12-48).32 patients were managed with closed reduction (Group I) and 23 patients were managed with open reduction and percutaneous pining (Group II).Two crossed pins were used for fracture stabilization in both treatment groups. Statistical comparisons among groups were done with Mann-Whitney $U$ tests, Pearson's Chi-square and independent samples $t$-test.

Results: Compared with fractures requiring open reduction, fractures aligned by closed reduction had a shorter time to union ( 7 weeks vs. 5.8 weeks $p=0.01$ ). Fractures aligned by closed reduction had a lesser ROM restriction (3.80 vs. $12.30, \mathrm{p}=0.03$ ) and better functional results $(P=0.03)$ than fractures requiring open reduction. In Group I, four (12.5\%) patients had a superficial pin track infection. Additionally, there were two (6.3\%) iatrogenic ulnar nerve injuries. By the fourth postoperative month, both nerve injuries had completely resolved. In Group II, two patients $(8.7 \%)$ had a superficial pin track infection. Two patients $(8.7 \%)$ had wound dehiscence in postoperative period. These wounds were healed with-in 3 weeks without any reconstructive surgery. These two patients and one other patient have undesirable cutaneous scar tissue formation. Also in Group II, there were two avascular necrosis of trochlea. Myositis ossificans did not occur in any of the patients. Baumann angle and carrying angle difference and functional results are better in closed reduction group but the difference was not statistically significant $(P>0.05)$.

Conclusions: We have observed a higher rate of complications with open reduction than with closed reduction group. We prefer posterior midline skin incision and triceps sparing approach for open reduction since it provides better visualization for both medial and lateral columns. Cosmetic concerns mentions by angular deformity of the arm, whereas the scar formation is not included. We think scar formation is also an important factor for cosmesis.

Significance: We observed significantly better results in the closed reduction group than in open reduction group. We observed significantly better results in the closed reduction group than in open reduction group. The former had better function, less cosmetic problems and a greater range of movement of the elbow. That's why we recommend treating posteromedial displaced Gartland Type III supracondylar fractures of the humerus as possible as by closed reduction and percutaneous fixation.

\section{0}

\section{MANAGEMENT OF VASCULARLY COMPROMISED SUPRACONDYLAR FRACTURES}

\author{
D. Louahem M'Sabah, A. Diméglio, M. Ali, P. Kelly
}

Purpose: To analyse all supracondylar fractures with major displacement presenting with acute vascular compromise and to propose an effective therapeutic strategy in a tertiary referral centre.

Methods: Three hundred patients aged 18 months to 14 years were treated for Larange stage IV supracondylar fracture of the humerus in the same centre. Acute vascular compromise was noted in 46 cases $(15 \%)$. The radial pulse was absent in all patients with two different contexts: "pink hand" with good distal perfusion in 41 cases $(13.5 \%)$ and "white hand" with ischemia in five cases $(1.5 \%)$. Nerve injury was associated in half of the cases, predominantly involving the medial nerve $(87 \%)$. Management with emergency reduction under anesthesia and stable percutaneous fixation, with lateral and medial wires via a minimal medial exposure to control the ulnar nerve, was performed in all cases. Results: $90 \%$ of the cases with vascular compromise had posterolateral displacement. Post reduction, the radial pulse was restored immediately in 28 cases and secondarily in 10 . The three remaining cases with an absent radial pulse but with a pink hand developed ischemia necessitating surgical exploration revealing incarceration of the brachial artery and medial nerve within the fracture site. Release of the brachial artery restored the radial pulse. The five cases of primary ischemia with absent pulse and a white hand were managed by emergency reduction followed by exploratory surgery and vascular repair which restored the radial pulse, excepting one case which required anterior fasciotomy. Angiographic studies were not required in any patient.

Conclusions: This study identified the following points: priority is required for reduction of these fracture and emergency stabilization; posterolateral displacement is associated with a higher risk of vascular complications; these injuries should be treated in a specialized centre; the absence of a radial pulse with a pink hand warrants repeated observation during the postoperative period; it is not an absolute indication for invasive investigation and surgical exploration; the absence of a pulse with a white hand requires surgical vascular exploration.

Significance: This large series of vascularly compromised supracondylar fractures allow us to propose an optimal management strategy. Failure to achieve a vascularized hand post reduction of a supracondylar fracture necessitates immediate surgical exploration \pm vascular repair by a suitably trained surgeon without the need of intra-operative angiography.

\section{1}

\section{THE RESULTS OF TREATMENT IN MONTEGGIA EQUIVALENT LESIONS IN CHILDREN}

\section{Guven, A. Eren, B. Kadıoglu, E. Eceviz, K. Ozkan}

Purpose: Monteggia fracture-dislocation was first classified by Bado as four basic types and two equivalent lesions. Afterwards Reckling extended these descriptions and described many different pathologies as equivalent lesion. Various treatment modalities and results were reported in the literature. We evaluated our 
conservative and surgical treatment results of Monteggia equivalent lesions in children.

Methods: Thirteen children (10 male, 3 female; mean age 7.7 years; range 4-13 years) treated for Monteggia equivalent lesions with at least 2 years follow-up were evaluated. Equivalent lesions were classified into three groups. Seven (54\%) patients had ulna diaphysis or olecranon fracture and radial neck fracture without radial head dislocation; four $(31 \%)$ patients had olecranon fracture and lateral or anterolateral radial head dislocation; two $(15 \%)$ patients had ulna proximal metaphysis fracture and lateral humeral condylar fracture with lateral radial head dislocation. Patients in the first group had Type 1 equivalent lesions as described by Bado. Second and third group patients were defined as Bado Type 3 equivalent lesions. One patient with Type 3 equivalent lesion had open fracture. Urgent debridement and irrigation was performed for this patient. Primarily close reduction was applied for the others. Adequate reduction with conservative treatment was achieved in eight $(62 \%)$ patients. The remaining four $(34 \%)$ patients were operated due to failure of close reduction for radial head dislocation or fracture; annular ligament interposition or rupture; displaced olecranon, metaphyseal ulnar or lateral humeral condylar fractures.

Results: Mean follow-up period was 4.1 years (range 2-7 years). One radial nerve palsy was determined before close reduction and healed in third month spontaneously. None of the patients had nonunion. The evaluation of the functional results was based on a grading system that described by Reckling according to the range of motion of the elbow. Functional results were good in all patients treated conservatively. Four patients treated surgically had good functional results; furthermore the result in open fracturedislocation was poor.

Conclusions: Conservative treatment with close reduction is the first choice of treatment in Monteggia equivalent lesion. In the event of failure in close reduction, surgical treatment becomes a current issue. In our study non-reducible radial head dislocation or fracture, annular ligament interposition or rupture, displaced olecranon or metaphyseal ulnar fracture and lateral humeral condylar fracture especially in patients with Type 3 equivalent lesions were the reasons for the surgery.

Significance: Different fracture patterns can be seen in Monteggia equivalent lesions. Accurate diagnosis of these lesions is essential for satisfactory results. Bado Type 3 equivalent lesions can occur with lateral humeral condylar fracture because of the similarity of trauma mechanism.

\section{2}

\section{REMANIPULATION IN PAEDIATRIC WRIST AND FOREARM FRACTURES}

\section{Taylor, A. McGuinness}

Purpose: To investigate the incidence and characteristics of remanipulated wrist and forearm fractures in children treated in a regional trauma centre.

Methods: A retrospective analysis of patient charts was undertaken to identify and quantify paediatric wrist and forearm fractures remanipulated in our unit within the past 3 years, and corresponding radiographs were reviewed to identify characteristics of both the initial fracture and the subsequent loss of reduction.

Results: Of 660 fractures, 29 were remanipulated $(4.4 \%)$, at a mean time of 12.8 days since the initial reduction (range 4 23 days). All fractures had been manipulated under general anaesthesia within $24 \mathrm{~h}$ of presentation and position maintained in appropriate casts. One child underwent flexible intramedullary nailing for diaphyseal fracture of the radius, all others were managed by remanipulation only. Based on fracture pattern, four distinct groups were identified: (a) Metaphyseal fracture of the distal radius and ulna with complete displacement ('pronator quadratus' injuries, 10 fractures, mean 8.6 years). All had some residual displacement after initial reduction, and unacceptable dorsal angulation was the predominant reason for remanipulation. (b) Metaphyseal fracture of the distal radius (7 fractures, mean 10.1 years). Recurrence of dorsal angulation was also the predominant reason for remanipulation. (c) Salter Harris II fracture of the distal radius with complete dorsal displacement ( 5 fractures, mean 10.1 years). Recurrence of initial displacement occurred despite complete initial reduction, and remanipulation was suboptimal in four of these. (d) Diaphyseal fracture of the middle/distal radius with variable degrees of displacement and angulation ( 7 fractures, mean 5.6 years). Of these fractures, none has resulted in significant deformity or disability, with considerable remodeling seen in both the sagittal and coronal planes.

Conclusions: Despite this small cohort, loss of reduction has been noted in distinct fracture patterns, and these demand careful initial manipulation and closer review.

\section{3}

\section{EXTERNAL FIXATION FOR COMMINUTED AND SPIRAL} FEMORAL FRACTURES : A BENEFIT TO THE CHILD ?

\section{Petratos, C. Skoura, G. Matsinos, J. Anastasopoulos}

Purpose: To evaluate the efficacy of external fixation for the treatment of comminuted and spiral femoral fractures in children and adolescents.

Methods: Between 1991 and 2004 twenty comminuted and eight spiral femoral fractures in 28 patients were treated with reduction and application of the unilateral Orthofix DAF external fixator. The average age for the 26 boys and 2 girls was 11.53 years (range, $8-17$ years). Three fractures were open with one grade II and two grade IIIA (Gustillo classification). The majority of the fractures were in the middle and distal third (24 of 28) and the most common mechanism of injury was a motor vehicle accident $(50 \%)$. There were 20 children with isolated injury and 8 children with multiple trauma. Intraoperative fluoroscopy was used to guide pin placement and fracture reduction. Postoperative care consisted of once daily pin care. Partial weight bearing as tolerated by patient comfort was begun within the first few days and healing process was determined on the basis of two-projection radiographs. Healing was considered sound when a bony bridge of at least three cortices had been seen on AP and lateral radiographs. At this point dynamization was performed and full WB was permitted. Length of time in the device was recorded as were any complications. AP and lateral radiographs that were taken postoperatively and at final review were assessed for angular deformities. At final follow-up we assessed hip and knee ROM, limb-length inequality and a history was obtained regarding pain, limp and activity level.

Results: Mean follow-up time was 43 months (range, 2867 months) and the average duration in the fixator was 93 days (range, 60-122 days). Sound healing was revealed on radiographs in a mean time of 54.8 days and all 28 fractures healed without additional operative intervention. Complications included 6 pin tract infections and 1 superficial wound infection that were all treated with oral antibiotics. At final follow-up average angula- 
tion at the fracture site was $3.1^{\circ}$ in coronal plane (range, $0-8^{\circ}$ ) and $3.7^{\circ}$ in sagittal plane (range, $0-10^{\circ}$ ). 18 patients had clinically equal leg lengths, 8 patients had $<1 \mathrm{~cm}$ inequality and 2 patients a discrepancy between 1 and $1.5 \mathrm{~cm}$. All children had excellent function at the time of final review.

Conclusions: External fixation is an effective and safe method for the treatment of spiral and comminuted fractures in childhood and adolescence. Careful attention must be paid to operative technique and post operative treatment in order to minimize complications.

Significance: Intramedullary nails, spica cast and plating, each for different reason, can not be used for the fixation of unstable fracture configurations in children older than 6 years old with open physes. Therefore ex-fix may be the best choice for comminuted and spiral femoral fractures and especially for the metaphyseal ones.

\section{4}

\section{IMMEDIATE INCORPORATED HIP SPICA CASTING IN PEDIATRIC FEMORAL FRACTURES: COMPARISON OF EFFICACY BETWEEN NORMAL AND HIGH RISK GROUPS}

\author{
L. Celebi, A. Bicimoglu, C. N. Aktekin, E. Aksahin, \\ H. H. Muratlı, H. Y. Yuksel, L. Celebi, O. Hapa
}

Purpose: Immediate hip spica casting is the most commonly used method for the treatment of pediatric femoral fractures. The main disadvantage of the method is unacceptable shortening (more than $25 \mathrm{ml}$ ) which may occur during the treatment. Buehler et all described the so called 'telescope test' inorder to identify the cases with a relatively high risk of unacceptable shortening. Based on this test, patients with an overriding of the fracture ends more than $30 \mathrm{ml}$ have a 20.4 times higher risk of unacceptable shortening when compared to ones with an overridin of less than $30 \mathrm{ml}$. This relatively higher risk of unacceptable shortening may be avoided by a hip spica cast which is incorporated to a distal femoral traction pin.

Methods: The study consists of 47 (26 boys, 21 girls) patients. Mean age was 4 (18 months-6year) years. Patients were divided into two groups according based on telescope test. Group I included the patients with a telescop test of more than $30 \mathrm{ml}$. Group II included the patients with a telescope test of $30 \mathrm{ml}$ or less. All patients were treated with a hip spica cast which is incorporated to a distal femoral traction pin within $8 \mathrm{~h}$ of the initial trauma. During cast treatment, patients were followed by weekly X-ray controls for the first month. Cast was removed on 45 days. Patients were assessed for unacceptable shortening and misalignment.

Results: Mean shortening during cast treatment was $2.9 \pm$ $5.1 \mathrm{~mm}$ on treated site. Shortening was defected in $16(88.9 \%)$, $(P<0.001)$ in Group I and in $7(24.1 \%)(P>0.05)$ patients in Group II. Significantly higer rate $(P<0.001)$ patients developed shortening in group I. But unacceptable shortening $(>25 \mathrm{~mm})$ did not develop in any patient in any group. There was no significant frontal plane malalingment in any groups $(P>0.05)$. Sagittal plane malalingment was significantly higher in Group I $(P<0.05)$

Conclusions: Hip spica cast which is incorporated to a distal femoral traction pin avoids unacceptable shortening and frontal plane malalingment in pediatric femoral fractures which even have a relatively high risk of unacceptable shortening based on the so called 'telescope test'. But the technique can not avoid sagittal malalingment.

Significance: The study demonstrates that unacceptable shortening which is the main problem in hip spica casting of pediatric femoral fractures, may be prevented with incorporated cast. this will certainly reduce the need for shifting to other more expensive and morbid treatment modalities.

\section{5}

\section{OUTCOME OF THE LOWER LIMB FRACTURES IN CHILDREN AND ADOLESCENTS: PRELIMINARY RESULTS}

\section{Ceroni}

Purpose: Fractures during growing years represent a major public health problem, as $25 \%$ of patients who consult in pediatric emergency services having a fracture. At the end of puberty, half of adolescents experienced one fracture, and 15-20\% of them sustained more than one lesion. Childhood fractures occur during the critical period of skeletal development and may result in suboptimal peak bone mass at the end of puberty. The primary aim of this prospective study is to determine the short-term and mid-term impact of lower limb fracture on bone mineral accretion and metabolism in this population.

Methods: 50 children (10-16 years) admitted for lower limb fractures and 50 healthy controls without history of fracture have been matched for gender, age, height, and weight. Bone Mineral Density (BMD) was measured by both dual energy absorptiometry (DXA-Lunar Prodigy ${ }^{\circledR}$ ) and portable peripheral DXA (Lunar Pixi $\left.{ }^{\circledR}\right)$. Measurements will include lumbar spine, bilateral hips and bilateral calcaneal BMD at fracture time, cast removal, 6 and 18 months. Others measures will include: anthropometrics; bone age by left hand X-ray; nutrition; physical activity monitoring; muscle strength.

Results: At baseline, raw trend analysis did not show any significant differences in bone measures between subjects with lower limb fracture and age-matched healthy controls. At the end of the orthopedic treatment (cast removal), there was a significant bone mineral decrement (until $-30 \%$ ) compared to baseline values. The bone mineral density was significantly correlated with the physical inactivity due to casting. The physical activity level by accelerometer during the orthopedic treatment was $60 \%$ lower in children with lower limb fracture, compared to healthy controls. Our preliminary results showed at 6 months an incomplete recovery of the BMD of the affected side compared to baselines values.

Conclusions: The preliminary results suggest that the group of fractured subjects have normal bone mineral quality before the first fracture. The immobilization for the lower limb fracture is responsible for an important local bone mineral decrement. This bone mineral decrement reaches $30 \%$ at the level of the calcaneus and $15 \%$ at the level of the proximal femur. At 6 months of the trauma, we note that the recovery of the bone mineral decrement is incomplete as there is still $10 \%$ of decrease of the baseline BMD values.

Significance: This work gives an outline of the importance of the bone loss with the course the treatment of the fracture and of the constitutional weakness which results from this and which explains the repetitive fractures that one can note in this group of patients. Finally this work shows the repercussions of a fracture over the muscular strength over the affected member. 
176

\section{ANALYSIS OF THE FACTORS AFFECTING FINAL RESULTS OF THE OPERATIVELY TREATED FEMORAL NECK FRACTURES IN CHILDREN AND ADOLESCENTS}

\section{S. J. Snela, G. Sokó, P. Jasiński, R. Piasek}

Purpose: Femoral neck fractures in children occur 130 times more seldom than in adult's population and amount to ca $1 \%$ of all pediatric fractures. The most common complication is avascular necrosis $(\mathrm{AVN})$ of the femoral head. There are also possible other complications following the growth cartilage injuries like leg shortening or deformity of the proximal femoral end. Purpose of the study was the treatment's results analysis of the 15 children and adolescent's femoral neck fractures.

Methods: 15 patients at a mean age of 12,7 years (5-17) presenting with femoral neck fractures were analyzed. 13 of them were operated primarily in our institution. Closed reposition were performed in 14 patients (open in 1), 12 fractures were stabilized using 2 cannulated screws ( 1 patient $-\mathrm{K}$ wires, $1-\mathrm{K}$ wires and screw). Skeletal or skin traction were applied before surgery in 7 patients. The bone scintigraphy was performed in 8 patients due to early diagnosing of the AVN. On the pre- and postoperatively $\mathrm{X}$-rays we have determined the type of fracture in Delbet classification and compared femoral-neck angle with the healthy side. In the final clinical examination ROM, the equality of leg's length and quality of walking were checked. The results were determined in the Harris hip score. We divided the patients into 3 groups that depended on the time of surgery: (1) operated up to $24 \mathrm{~h}$ after injury (6 patients), (2) 24-72 h post fracture (5), (3) after $72 \mathrm{~h} \mathrm{4}$ ). Results: 10 fractures were classified to type 2, 3 fractures to type 3 according to Delbet scale (X-rays of 2 patients are missed). The final outcome after $7-163$ months (57 on average) could be precised at 13 patients. Significant ROM decreasing was confirmed in 4 patients. The highest restriction was noticed in internal rotation (on average $14^{\circ}$ ). Other motions were slightly restricted. Inequality of limb's length amounted $0,5 \mathrm{~cm}$ on average. The average Harris hips score amounted 92.58 (76-100). The final results were assessed as very good at 8 patients, good at 4 and moderate in 1 patient. The detailed data separately for each group of patient are as following:

In general Harris scale: Possible score-100, Group 1-99.1, Group 2-91.5, Group 3-81.

Pain: Possible score-44, Group 1-43.3, Group 2-41, Group 326.7.

Function: Possible score-47, Group 1-47, Group 2-44.5, Group 3-45.4.

Deformity: Possible score-4, Group 1-3.8, Group 2-3.8, Group 3-4.

ROM: Possible score-5, Group 1-5, Group 2-3.7, Group 3-5.

AVN were suspected in 3 patients, 2 of them were scintigraphically confirmed.
Conclusions: Worse treatment's results were achieved at patients operated later than 3 days after injury. In the Harris hip score pain was the most influenced factor.

Significance: Most important to avoid AVN of the femoral head is the quick reposition and adequate stabilization.

\section{7}

\section{AVASCULAR NECROSIS ASSOCIATED WITH FRACTURES OF THE FEMORAL HEAD AND NECK IN CHILDREN}

\section{I. Bytyqi, A. Grazhdani, A. Pustina, A. Tolaj, H. Qorraj-Bytyq}

Purpose: To study the complications of the traumatic proximal femur fractures especially femoral head necrosis in children including and two cases with gunshot injuries.

Methods: This is a retrospective study of children with traumatic fractures of femoral neck and head over a 10 year period 19912001. Eighteen children with traumatic fractures of femoral neck (average age 9 years and 8 months) were included with a followup of 5-11 years (mean 7.8 years). A traffic accidents, fall from height were the main causative factors and two cases caused due to gunshot injuries. In the first case the penetrating missile had the entry wound in the inguinal region and exit in retrotrochanteric region. The bullet hole passed through the femoral neck very close to the proximal femoral physis. In the second cases with gunshot injury had the enter point in the anterior femoral region and exit wound in the sacral region. The bullet hole in the second gunshot case passed through the femoral head. Non operativ treatment has been applied in 6 children with undisplaced fractures. For all displaced fractures decompression of the hip joint by aspiration and closed reduction and internal fixation was performed within $24 \mathrm{~h}$. All patients were reexamined at least 5 years after injury. Results: They were: 1 patient with type I fractures, 7 patients with type II fractures, 6 patients with type III fractures and 4 patients with type IV fractures according Delbet's classification. Only 10 patients $(55.5 \%)$ of all osteosyntheses were performed on the day of hospital admission. In $5(27.7 \%)$ of cases, there was another significant associated injury to the chest, head or abdomen. 16 patients had good results and 2 had fair results using Ratliff criteria. In 2 cases AVN was Type I with diffuse, complete head involvement, and collapse. At last follow-up, 2 of 18 children $(11.2 \%)$ suffered avascular femoral head necrosis and premature epiphyseal closure, coxa vara and breve, and leg length discrepancy more than $2 \mathrm{~cm}$.

Conclusions: Nondisplaced fractures do not have the same incidence of avascular necrosis as displaced fractures. This fact further support the concept that the magnitude of initial fragment separation is major determinant of vascular damage.

Significance: Presentation of outcome of femoral head and neck injuries. 